\title{
MEASURING PATIENT SAFETY IN NEW ZEALAND PUBLIC HOSPITALS
}

BY

NICHOLAS BOWDEN

\begin{abstract}
A thesis
submitted to the Victoria University of Wellington in fulfilment of the requirements for the degree of Masters of Commerce
\end{abstract}

Victoria University of Wellington 2016 


\begin{abstract}
In New Zealand the Ministry of Health recognises quality of care as an integral part of a high performing health system and identifies patient safety as one of the key dimensions of quality. Over recent years a greater emphasis has been placed on improving patient safety mostly as a result of increased awareness around the frequency of medical error and resulting economic cost. However tools used to measure patient safety are limited. In particular the use of hospital administrative data to measure patient safety is scarce and existing safety measures often ignore one of the major issues confronting comparative analyses of hospital safety, risk adjustment to control for the differences in populations hospitals serve.
\end{abstract}

The objective of this research is to develop comparable measures of patient safety for New Zealand public hospitals. It uses risk adjustment strategies applied to the Agency for Healthcare Research and Quality (AHRQ) Patient Safety Indicators (PSIs) with New Zealand hospital administrative data, the National Minimum Dataset 2001 to 2009. The research employs econometric techniques to address risk adjustment of the PSIs, utilising existing AHRQ models but adapting and re-estimating them with New Zealand administrative data.

The findings from the research indicate that to use the AHRQ PSIs as measures of hospital patient safety in New Zealand, risk adjustment should first be employed to ensure measures are comparable across hospitals and over time. Overall, although the impact of risk adjustment appears to be minor, it has relevance and this should be recognised. Relative hospital performance is affected by risk adjustment. In particular, it has the greatest impact on those hospitals with poor rankings. The research takes us a step closer to being able to confidently measure patient safety and quality of care in New Zealand public hospitals in an innovative way. 


\section{Table of Contents}

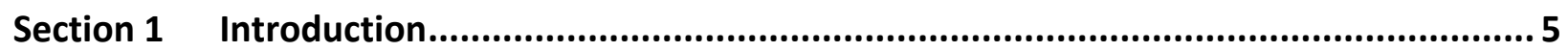

Section 2 Literature Review ............................................................................... 9

2.1 Quality in Health Care: Patient Safety \& Patient Safety Measurement ........................ 10

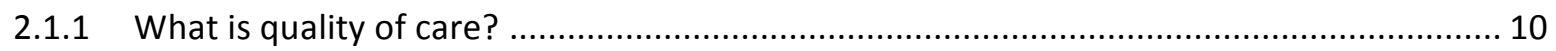

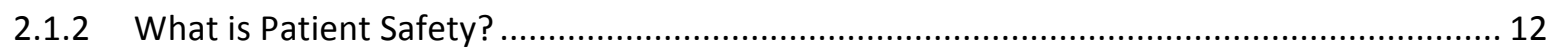

2.1.3 Importance of Patient Safety and Quality of Health Care .................................................. 14

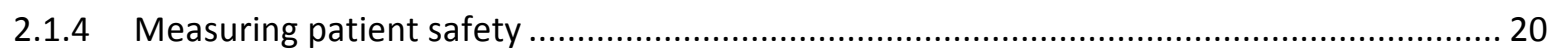

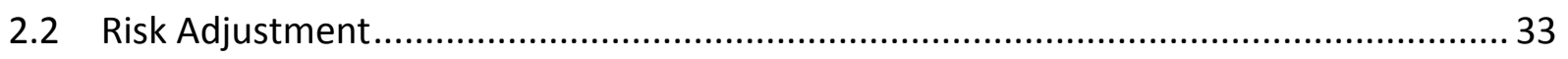

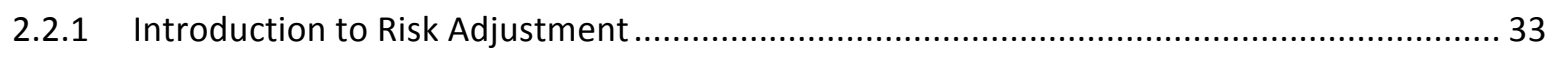

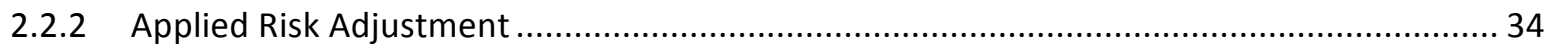

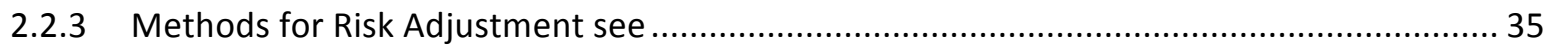

2.2.4 Empirical Evaluation of Risk Adjustment Models............................................................ 37

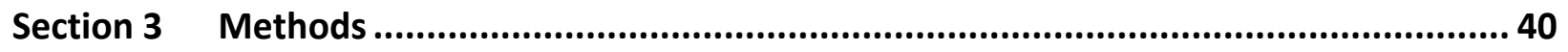

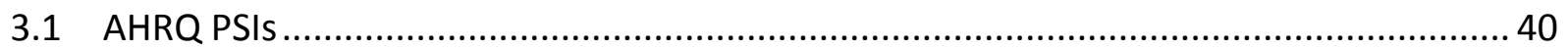

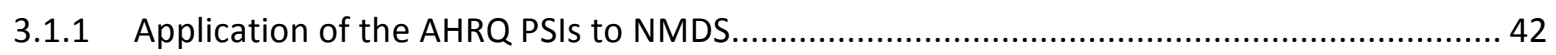

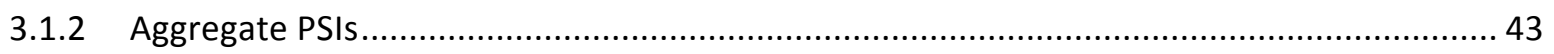

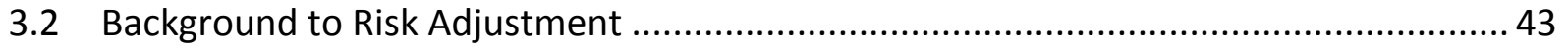

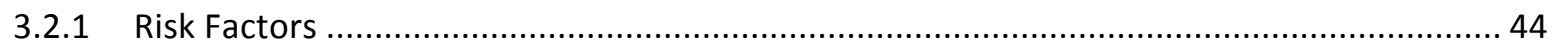

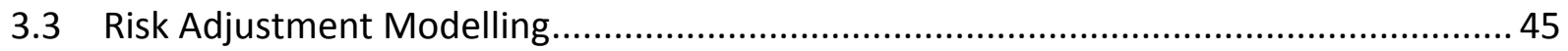

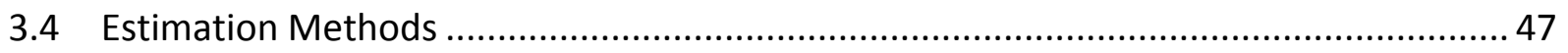

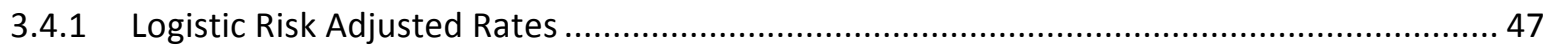

3.4.2 Ordinary Least Squares (OLS) Risk Adjusted Rates.......................................................... 49

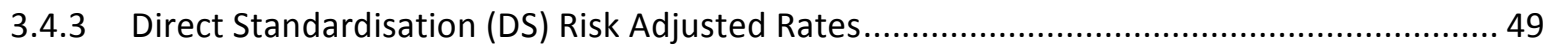

3.5 Comparing Alternative Risk Adjustment Methods .................................................... 50

3.6 Empirical Evaluation of Risk Adjustment Models ...................................................... 51

3.7 Analysis of Hospital Level Risk Adjusted Rates ......................................................... 52

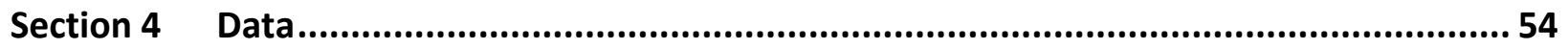

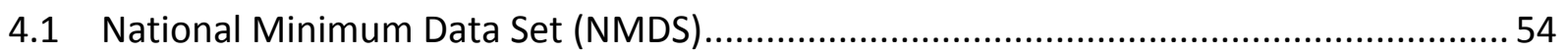

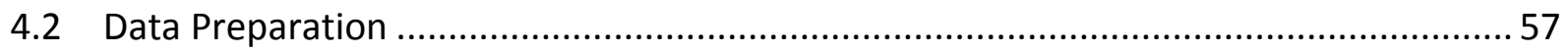

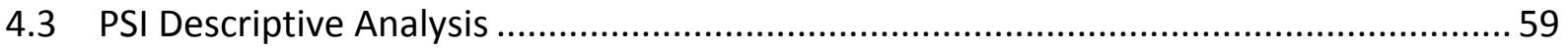


4.4 Cost of Adverse Events

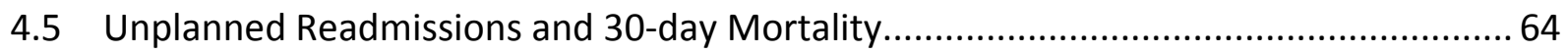

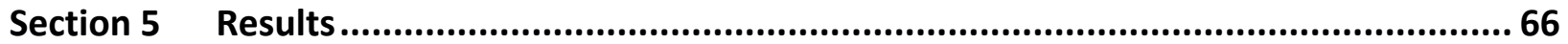

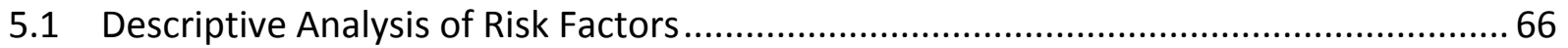

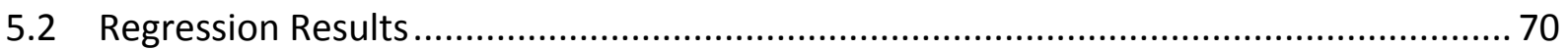

5.2.1 Background to Interpreting Logistic Regression Results .................................................. 70

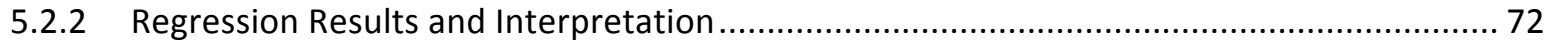

5.3 Empirical Evaluation of the Risk Adjustment Models ................................................. 77

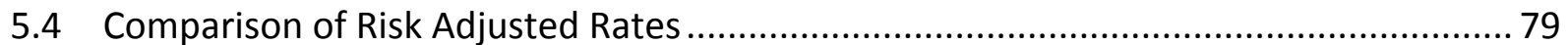

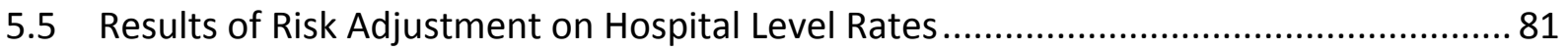

5.5.1 Effects of Risk Adjustment On Hospital Rates Across Incremental Models ........................ 81

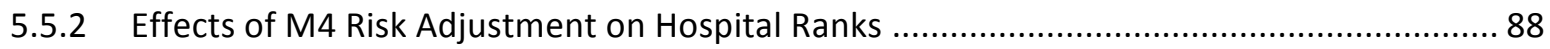

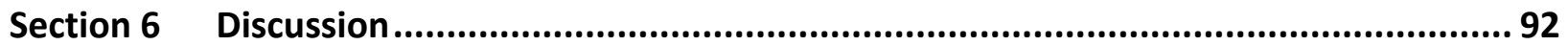

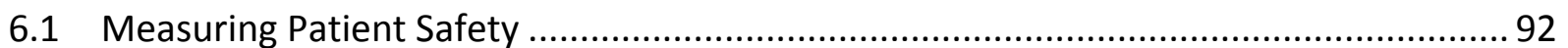

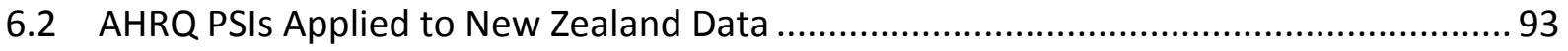

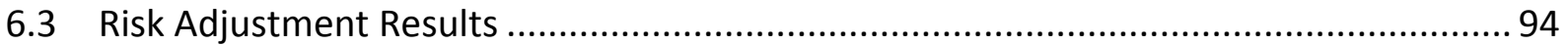

6.4 Limitations of current work and possibilities for future work .................................... 97

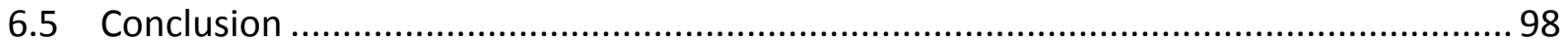

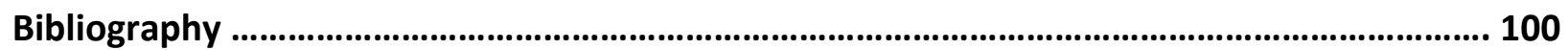

Appendix A

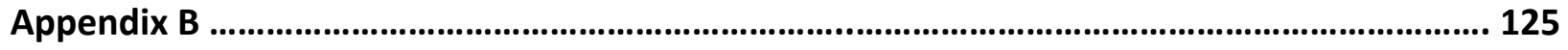

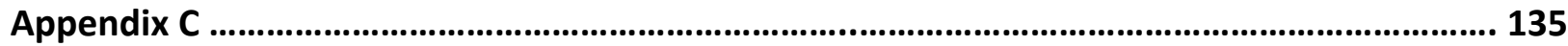

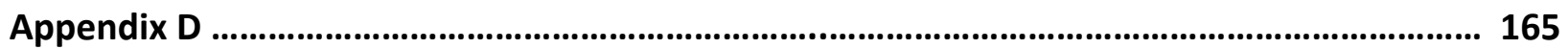

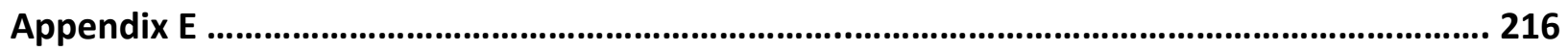




\section{Section 1 Introduction}

The performance of the health sector is significant to the overall social well-being and economic performance of a country. New Zealand health care expenditures are rising and for the $2015 / 16$ financial year government spending on public health accounted for circa $\$ 15.6$ billion, or $6.9 \%$ of New Zealand's Gross Domestic Product (Reserve Bank of New Zealand 2015, Treasury 2015). Fuelled by rising health care expenditures, pressures are mounting to reduce health care costs and increase efficiency, while at the same time improve standards of care. As a result it has become increasingly important for policy makers to have a good understanding of how well the health sector is performing. This requires measurement of key performance indicators.

One key element of health care provision and performance is quality of care (Kohn, J. M.Corrigan et al. 2000, McDonald, Romano et al. 2002). Quality of care is a multidimensional construct and patient safety is the cornerstone of high quality health care. Patient safety can be broadly defined as the prevention of avoidable harm to patients during health care. The World Health Organization (WHO) recognises patient safety as a "fundamental principle of patient care and a critical component of quality management" (World Health Organization 2006 , pg. 4). In New Zealand the Ministry of Health (MoH) has identified patient safety as one of the key dimensions of quality in the health care sector (Ministry of Health 2003).

Adverse events, generally defined as harm caused by health care, are a key measure of patient safety. Internationally, studies show adverse events are frequent amongst hospital admissions (Brennan, Leape et al. 1991, Leape, Brennan et al. 1991, Wilson RM, Runciman WB et al. 1995, Thomas, Studdert et al. 2000, Vincent, Neale et al. 2001, Baker, Norton et al. 2004). United States figures suggest between 44,000 and 98,000 Americans die each year as a result of medical error (Brennan, Leape et al. 1991, Leape, Brennan et al. 1991, American Hospital Association 1999, Thomas, Studdert et al. 2000). A New Zealand study conducted in 1998 concluded that 12.9 percent of all public hospital admissions were associated with adverse 
events, and approximately 35 percent of these were judged highly preventable (Davis P, LayYee $R$ et al. 2001).

Adverse events also incur significant economic costs (Johnson WG, Brennan TA et al. 1992, Thomas, Studdert et al. 1999, Vincent, Neale et al. 2001, Zhan C and Miller M 2003). One of the first studies to estimate costs attributable to medical error was conducted by Johnson et al (1992) in New York State. They found total costs of \$878 million (in 1989 dollars); \$161 million in medical care costs, $\$ 276$ million in lost wages, and $\$ 441$ million in lost productivity based on a random sample of 794 admissions with adverse events. Extrapolation of the results shows state-wide per capita costs of adverse events to be \$189 in New York State. In New Zealand up to $30 \%$ of public hospital expenditures have been attributed to treating adverse events (Brown, McArthur et al. 2002).

While the issue of the patient safety problem has been recognised, there is a lack of useful information on the quality of health care providers in terms of safety of care. McClellan and Staiger (1999) identify four core reasons for this. First, collecting timely and relevant data is difficult. Many studies which attempt to measure the quality and safety of health care providers are one-off studies, extremely resource intensive, and they have significant delays between patient outcomes and actual measurement. Second, multidimensionality of the construct must be addressed. Even when one restricts the view of patient safety to medical error and adverse events, a range of adverse outcomes can occur ${ }^{1}$. Therefore a number of adverse events need to be considered in any quality evaluation. Third, the comparability of safety measures poses a challenge since hospitals treat a variety of patients with differing casemix. Variation in quality across hospitals is likely to reflect both the care provided as well as differences in casemix, and therefore both must be considered in deriving measures of patient safety. This is generally referred to as risk adjustment. Fourth, the reliability of some patient safety measures is also a concern. Safety measures are generally regarded as inherently noisy measures of hospital quality (McClellan and Staiger 1999). This can be explained by the fact that the measurement of specific adverse events pose specific challenges; small sample sizes,

\footnotetext{
${ }^{1}$ Examples include falls, hospital acquired infections, decubitus ulcers, and deep vein thrombosis or pulmonary embolism.
} 
the relative infrequency of adverse outcomes, measurement error, and the large number of additional factors other than hospital quality that could influence patient outcomes.

This thesis aims to develop patient safety measures for New Zealand public hospitals and in the process address the first three of these four issues. More specifically the research aims to develop hospital safety measures that are able to be derived at low cost from hospital administrative data periodically and systematically. These aim to be reflective of quality of health care services along multiple dimensions, and risk adjusted so comparable across providers and over time. The issue of reliability is left for future research because of the time constraints in dealing with this complex issue.

The research uses the Agency for Healthcare Research and Quality (AHRQ) Patient Safety Indicators (PSIs) ${ }^{2}$ with nine years of New Zealand hospital administrative data as alternative metrics of hospital safety and quality to those already used in New Zealand (AHRQ 2007, AHRQ 2008). The AHRQ PSIs consist of 20 provider-level indicators and are used with administrative data to identify potentially preventable complications and iatrogenic events for patients treated at hospitals. Each of the PSIs focuses on separate pre-defined potential adverse events (AEs) and areas of patient safety, but all reflect in various ways the multidimensional concept of hospital safety and quality. They work by applying algorithms to discharge level hospital administrative data and flagging whether or not an AE potentially occurred, based on the clinical coding of the individual patient discharge.

The study explores and evaluates alternative risk adjustment models that employ incremental sets of risk factors. Logistic based risk adjustment is conducted on discharge level data through which hospital level risk adjusted PSI rates are constructed. The impact of risk adjustment on hospital level rates of adverse events is then assessed.

The findings from the research indicate that in order to use the AHRQ PSIs as comparative measures of patient safety for New Zealand hospitals, risk adjustment is required. Analysis indicates that the most appropriate models for risk adjustment are those which contain a range

\footnotetext{
${ }^{2}$ The AHRQ PSIs are discussed in detail in Section 2 and 3.
} 
of risk factors: gender, age, ethnicity, deprivation level, diagnoses, and comorbidities. Such risk adjustment models have greater predictive power and are considered most appropriate based on empirical evaluation methods. In general, age, gender, ethnicity, and deprivation tend to account for variation in hospital rates while DRGs and comorbidities mask them. Overall the impact of risk adjustment is relatively small, however there is some impact and it must be acknowledged that that is important. In particular, risk adjustment appears to have the greatest impact on those hospitals with poor rankings.

The document is structured as follows. Section 2 reviews the relevant literature relating to patient safety and quality of care, and risk adjustment. Section 3 outlines the methods employed. These include how the AHRQ indicators are applied to New Zealand data, the methods employed for risk adjustment, and how the impact of risk adjustment on hospital performance is assessed. Section 4 describes the data used in the study and provides descriptive analysis on the AHRQ PSIs. Section 5 presents the results of the study. This includes a descriptive analysis on the empirical motivation for risk adjustment, results from logistic regressions for risk adjustment, empirical evaluation of risk adjustment models, and the impact of risk adjustment on hospital performance. Section 6 provides a discussion of the research, future considerations, and overall conclusions. 


\section{Section 2 Literature Review}

Quality has become an integral element of health care provision and patient safety is a fundamental aspect of quality of care (Aspden, Corrigan et al. 2004). In order to achieve safety (or quality) improvements in health care, there must first be accurate measurement (World Health Organization 2009). In turn, meaningful measurement of patient safety must recognise several key challenges: the availability of timely and relevant data, multidimensionality of patient safety, comparability or risk adjustment of patient safety measures, and reliability of some patient safety measures (McClellan and Staiger 1999).

Section 2.1 reviews the literature on quality in health care and subsequently on patient safety as arguably its most significant component. Section 2.1.1 summarises definitions and frameworks for quality of care highlighting patient safety as a fundamental component. Section 2.1.2 narrows the scope of research to focus specifically on the key dimension of patient safety. The importance of patient safety, largely driven by the empirical literature quantifying the issue in terms of both human and economic cost, is summarised in 2.1.3. The sub-section also highlights the institutional focus on patient safety and quality in health care to illustrate the growing awareness of their importance worldwide. The four common methods of measurement are reviewed in 2.1.4. A specific focus is placed on a world leading set of statistical indicators known as the AHRQ PSIs which are used throughout the remainder of the research. Because the PSIs use New Zealand hospital administrative data, they provide one avenue for addressing the issue of the availability of timely and relevant data. Furthermore, the PSIs cover 20 different aspects of patient safety and therefore go some way to recognising the multidimensionality of the issue.

Section 2.2 focuses on risk adjustment to ensure patient safety measures are comparable across hospitals and over time. Section 2.2 .1 begins by introducing risk adjustment, describing its importance in the context of outcome based hospital quality comparisons, and discusses methods of devising a risk adjustment strategy. Section 2.2.2 provides some examples in the literature where risk adjustment has been used. Section 2.2 .3 discusses the 
main methods for risk adjustment covered in the literature. Lastly, Section 2.2.4 reviews empirical methods for risk adjustment model evaluation.

\subsection{Quality in Health Care: Patient Safety \& Patient Safety Measurement}

\subsubsection{What is quality of care?}

Definitions and frameworks for quality of care are by no means unified in the literature. However, in general, quality of care is described as a complex construct, with multiple dimensions, and one which is central to the provision of health care around the world (Blumenthal 1996). A common characteristic of any view of quality of care is that patient safety is a fundamental component (Corrigan JM, Donaldson MS et al. 2001, Aspden, Corrigan et al. 2004, Darzi 2008). The Institute of Medicine (IOM) goes as far as to say patient safety is "indistinguishable from the delivery of quality health care" (Aspden, Corrigan et al. 2004, pg. 5).

One of the earliest and more influential definitions of quality of care comes from Donabedian who defines quality of care as, "care which is expected to maximize an inclusive measure of patient welfare, after one has taken account of the balance of expected gains and losses that attend the process of care in all its parts" (Donabedian 1980). This was followed some years later by the IOM's definition, "the degree to which health services for individuals and populations increase the likelihood of desired health outcomes and are consistent with current professional knowledge" (Lohr and Schroeder 1990, pg. 707). The fundamental difference between the two is that the IOM narrows the definition to one of improving patient outcomes from that of total patient welfare.

Rather than a formal definition the WHO employs a quality of care framework based on six dimensions of quality suggesting health care should be effective, efficient, accessible, acceptable/patient-centred, equitable, and safe (World Health Organization 2006). Effective care relates to providing best practice health care resulting in improved outcomes based on need. Efficiency refers to optimally using scarce resources in health care delivery. Accessible care necessitates that health care is received in a timely manner, regardless of geographical location, and provided at an appropriate level based on medical need. 
Acceptable and patient-centred care requires an individual's culture and preferences be taken into account. Equitable care demands that care of equal quality be provided regardless of differences in patient characteristics. Finally, safety means minimising risks and harm to health care users.

Other institutional frameworks for quality of care vary although each includes safety as a core dimension. The IOM's framework is developed around six aims for quality improvement citing health care should be: safe, effective, patient centred, timely, efficient, and equitable (Corrigan JM, Donaldson MS et al. 2001). In the United Kingdom, the National Health Service (NHS) and the Research and Development Cooperation (RAND) highlight patient safety as the primary criterion for quality care (Department of Health 2008, Nolte 2010). The NHS includes two further criteria, patient experience and effectiveness of care, while RAND differs only by including access of care.

The National Roundtable on Health Care Quality, convened by the IOM in 1998, further categorises health care quality problems into those of; underuse, overuse, and misuse. Underuse is described as the failure to provide needed treatments while overuse is the provision of unneeded care. Misuse refers to preventable complications from health care (Chassin and Galvin 1998). As a result, misuse became a common reference point for the link between quality of care and patient safety. However, more recently underuse and overuse have also been linked to patient safety (Leape and Berwick 2005).

New Zealand's MoH has adopted the IOM framework adapting it for the New Zealand environment (Ministry of Health 2003). The core principles of the Treaty of Waitangi provide the foundations of the framework from which five dimensions of quality of care are built: people-centred, access and equity, safety, effectiveness, and efficiency. The dimension of equity and access is particularly important as it addresses obligations under the Treaty specific to Maori health.

Donabedian provides perhaps the most well-known framework for quality of care outside of institutional frameworks. (Donabedian 1966). He conceptualises quality of care into three components: structure, process, and outcome. Structure centres on the context in 
which care is provided. Process is the combination of actions that make up that care. Outcome is defined as, "those changes, either favourable or adverse, in the actual or potential health status of persons, groups or communities that can be attributable to prior or concurrent care" (Donabedian 1985, pg. 256). Therefore while Donabedian's framework does not specifically mention patient safety, it is implied by the causal link of health care provision and adverse outcomes.

\subsubsection{What is Patient Safety?}

Despite varying definitions of quality of care, an underlying theme emerges that puts patient safety at the forefront. Distinguishing patient safety from quality of care has been a challenge for some. According to Vincent, with patient safety the focus is on health care that is harmful, as opposed to just not of good standard (Vincent 1997). It is this idea of harm, and the fact that there is too much harm, that is at the heart of the field of patient safety.

There are numerous definitions of patient safety. Most focus on the issue of prevention of medical error and the avoidance of patient harm. The IOM defines patient safety simply as "the prevention of harm to patients" (Aspden, Corrigan et al. 2004, pg. 5) and the WHO as "the absence of preventable harm to a patient during the process of health care" (World Health Organization 2012). The AHRQ's definition is consistent with these but also highlights the potential for error, "the absence of the potential for, or the occurrence of, health care associated injury to patients created by avoiding medical errors as well as taking action to prevent errors from causing injury" (AHRQ 2003).

In 2006 the National Quality Forum attempted to bring further clarity and definition into the idea of patient safety in order to create a standardised patient safety taxonomy. They define patient safety as, "the prevention and mitigation of harm to patients" and in turn define harm as, "any physical or psychological injury or damage to the health of a person, including both temporary and permanent injury" (National Quality Forum 2006, pg. 8). Similarly, Pronovost and Thompson et al. review the definitions of patient safety (Pronovost, Thompson et al. 2005, pg. 8). They adhere to the AHRQ definition of patient 
safety and clarify that medical error is the result of care process which either results in, or has the potential to result in, patient harm. These can be attributed to both errors of commission (action that is taken) and omission (action not taken). The authors add further clarity by defining incidents as "unexpected or unanticipated events or circumstances not consistent with the routine care of a particular patient, which could have, or did lead to, an unintended or unnecessary harm to a person, or a complaint, loss, or damage". In turn their definition of a near miss is "an occurrence of an error that did not result in harm", an adverse event, "injury resulting from a medical intervention", and a preventable adverse events, "harm that could be avoided through reasonable planning or proper execution of an action".

Emanuel and Berwick et al. also attempt to synthesise the intellectual history and definitions (Emanuel, Berwick et al. 2008). In this model patient safety is regarded as a discipline that applies safety science methods in order to achieve a trustworthy system of health care delivery. It is defined as, "an attribute of health care systems that minimises the incidence and impact of adverse events and maximizes recovery from such events". The authors have created an overarching model of patient safety founded on these definitions that divides health care systems into four domains. These are providers of care, recipients of care, the health care delivery process, and methods for feedback and improvement.

The authors claim their model is consistent with other existing frameworks that underpin patient safety. For example it compares with Deming who discusses the wider notion of "deep knowledge" of quality design which requires an understanding of the system, variations in performance, change as a source of knowledge, and the psychology of the people working the organisation (Deming 1986). Each of these elements drives quality improvement and fits within the Emanuel and Berwick et al. model's domain of "methods."

The three components that Donabedian uses to conceptualise quality of care; structure, process, and outcomes, are also consistent with patient safety. These categories can be viewed as intersecting with each of the four domains in the Emanuel and Berwick et al. model. 
Vincent identifies seven key elements that affect patient safety. These relate to organisation and management, work environment, team, task, individual, patient characteristics, and the external environment (Vincent 2006). These are distributed among the three domains: systems for therapeutic action, the people who work in health care, and the people who receive it or have a stake in its availability.

While it would be good to have consensus on patient safety one could argue much of this is simply semantics and in many practical senses the details of the definition is of secondary importance. However, without question, review of the literature shows that despite the variation in definitions and frameworks there are some clear commonalities: Harm is at the forefront of patient safety, medical error must be minimised, and the core measure of harm is that of adverse events.

\subsubsection{Importance of Patient Safety and Quality of Health Care}

The patient safety problem has received growing attention in recent years largely driven by the empirical literature quantifying the issue. As a result the institutional focus has intensified. This sub-section begins by reviewing the seminal empirical literature, identifying the magnitude of the patient safety problem, and highlighting both the frequency and economic cost of medical error. It also highlights the institutional focus on patient safety and quality in health care to illustrate the growing awareness and their importance worldwide.

\section{Empirical Evidence of the Patient Safety Problem}

Studies reveal that between 2.9 and 16.6 percent of patients admitted into hospitals experience one or more adverse events, a high percentage are preventable, and in some cases lead to death (Brennan, Leape et al. 1991, Leape, Brennan et al. 1991, Wilson RM, Runciman WB et al. 1995, Thomas, Studdert et al. 2000, Vincent, Neale et al. 2001, Baker, Norton et al. 2004). See Table 1 for a brief summary of the studies identifying the prevalence of adverse events. 
The most widely cited piece of work on the prevalence of adverse events is the Harvard Medical Practice Study. This was undertaken in New York State in 1984 and reviewed 30,121 hospital discharges across 51 hospitals (Brennan, Leape et al. 1991, Leape, Brennan et al. 1991). The study found that adverse events occurred in 3.7 percent of hospitalisations with 58 percent judged to be attributable to error (i.e. preventable), and 13.6 percent leading to death. The authors concluded that although improvements in medical knowledge will contribute to prevention of many adverse events, a high proportion of events are attributable to human error and are thus preventable.

A subsequent study conducted in Colorado and Utah in 1992 across 15,000 discharges from a representative sample of 28 hospitals across both states broadly echoed the Harvard Medical Practice Study findings (Thomas, Studdert et al. 2000). This study found that 2.9 percent of all discharges in each state were associated with an adverse event. Of these 53 percent were judged preventable, and 6.6 percent resulted in deaths.

Results from the above studies are the basis for one of the key findings in the landmark IOM report, To Err is Human: Building a safer health care system. The study found that between 44,000 and 98,000 Americans die each year as a result of medical error, numbers which mean that, even at the lower estimate, medical error is the $8^{\text {th }}$ leading cause of death in the United States (Kohn, J. M.Corrigan et al. 2000).

In Australia, the Quality in Australian Health Care Study was conducted based on the same methodology employed by the Harvard Medical Practice Study (Wilson RM, Runciman WB et al. 1995). This investigated 14,179 records of patient admitted to 28 hospitals in New South Wales and South Australia in 1992. Researchers found that 16.6 percent of admissions were associated with an adverse event; 51 percent of these were considered preventable, and 4.9 percent resulted in death. 
Table 1: Summary of results from empirical studies of adverse events in hospitals

\begin{tabular}{|c|c|c|c|c|}
\hline Study & Setting & $\begin{array}{l}\text { Exclusion of } \\
\text { low risk } \\
\text { patients }\end{array}$ & Adverse event definition & Adverse event rates \\
\hline $\begin{array}{l}\text { Brennan et } \\
\text { al., Leape et } \\
\text { al. }\end{array}$ & $\begin{array}{l}51 \text { New York } \\
\text { Hospitals, } \\
n=30195(1984)\end{array}$ & No & $\begin{array}{l}\text { Unintended injury or } \\
\text { complication that resulted in } \\
\text { disability, death or prolonged } \\
\text { hospital stay and was caused } \\
\text { by health care management } \\
\text { rather than underlying disease } \\
\text { process }\end{array}$ & $\begin{array}{r}3.7 \% \text { overall rate } \\
58 \% \text { preventable } \\
13.6 \% \text { led to death }\end{array}$ \\
\hline Thomas et al. & $\begin{array}{l}28 \text { Hospitals in } \\
\text { Utah and } \\
\text { Colorado (1992) } \\
n=14700\end{array}$ & No & $\begin{array}{l}\text { Injury caused by medical } \\
\text { management rather than } \\
\text { underlying disease process and } \\
\text { resulted in prolonged length of } \\
\text { stay or disability at discharge }\end{array}$ & $\begin{array}{l}2.9 \% \text { overall rate } \\
53 \% * \text { preventable } \\
6.6 \% \text { led to death }\end{array}$ \\
\hline Wilson et al. & $\begin{array}{l}28 \text { hospitals in } \\
\text { NSW and SA, } \\
n=14179(1992)\end{array}$ & $\begin{array}{l}\text { Partial (did not } \\
\text { exclude } \\
\text { obstetrics } \\
\text { admissions) }\end{array}$ & $\begin{array}{l}\text { Unintended injury or } \\
\text { complication that resulted in } \\
\text { disability, death or prolonged } \\
\text { hospital stay and was caused } \\
\text { by health care management } \\
\text { rather than underlying disease } \\
\text { process }\end{array}$ & $\begin{array}{l}16.6 \% \text { overall rate } \\
51 \% \text { preventable } \\
4.9 \% \text { led to death }\end{array}$ \\
\hline Vincent et al. & $\begin{array}{l}2 \text { hospitals in } \\
\text { London, } \\
\mathrm{n}=1014 \text { (1999- } \\
2000)\end{array}$ & No & $\begin{array}{l}\text { Unintended injury caused by } \\
\text { medical management rather } \\
\text { than by disease process }\end{array}$ & $\begin{array}{r}10.8 \% \text { overall rate } \\
48 \% \text { preventable } \\
8 \% \text { led to death }\end{array}$ \\
\hline Baker et al. & $\begin{array}{l}20 \text { Canadian } \\
\text { hospitals, } \\
n=3745(2000)\end{array}$ & Yes & $\begin{array}{l}\text { Unintended injury or } \\
\text { complication that resulted in } \\
\text { disability, death or prolonged } \\
\text { hospital stay and was caused } \\
\text { by health care management } \\
\text { rather than underlying disease } \\
\text { process }\end{array}$ & $\begin{array}{l}7.5 \% \text { overall rate } \\
36.9 \% \text { preventable } \\
20.8 \% \text { led to death }\end{array}$ \\
\hline Davis et al. & $\begin{array}{l}13 \text { hospitals in } \\
\text { New Zealand, } \\
n=6579 \text { (1998) }\end{array}$ & $\begin{array}{l}\text { Partial (did not } \\
\text { exclude } \\
\text { obstetrics } \\
\text { admissions) }\end{array}$ & $\begin{array}{l}\text { Unintended injury or } \\
\text { complication that resulted in } \\
\text { disability, death or prolonged } \\
\text { hospital stay and was caused } \\
\text { by health care management } \\
\text { rather than underlying disease } \\
\text { process }\end{array}$ & $\begin{array}{l}12.9 \% \text { overall rate } \\
35 \% \text { preventable }\end{array}$ \\
\hline
\end{tabular}

* Number taken from alternative paper based on the same study

Sourced from (Baker, Norton et al. 2004)

Additional studies have been conducted in Britain and Canada, each largely reflecting the results of the previous research. In Britain a review based on 1,014 admissions from two 
acute hospitals in London found that 10.8 percent of admissions experienced an adverse events; 48 percent of these were considered preventable, with eight percent judged to contribute to death (Vincent, Neale et al. 2001). In Canada, 3,745 patient medical records were reviewed from 20 hospitals across five provinces and it was found that 7.5 percent were associated with one or more adverse events. Of these 36.9 percent were deemed to be avoidable and 20.8 percent resulted in death (Baker, Norton et al. 2004).

An equivalent New Zealand study on adverse events in New Zealand public hospitals reviewed the medical records of 6,579 patients admitted into 13 public hospitals in 1998 (Davis P, Lay-Yee R et al. 2001). The study based its methodology on that of the Harvard Medical Practice Study and found that 12.9 percent of all admissions were associated with adverse events, and approximately 35 percent of these judged highly preventable.

The discrepancy between the results of the Australian study and the results from the United States based studies prompted research into the reasons for the difference. One study reanalysed the Australian data using the exact methodology employed by the Colorado/Utah study and found the rate of adverse events fell from $16.6 \%$ to $10.6 \%$ (Localio, Hamory et al. 1997). The study identified five areas of methodological difference that accounted for the reduction including the thresholds used to define medical causation. It concluded that the remaining three-fold difference could be put down to a combination of differences in quality of care and variation in the content of medical records and the behaviour of the reviewer. Other researchers found that with respect to serious adverse events the two studies were similar and the differences therefore were related to adverse events involving minor disabilities. They concluded that, rather than differences in quality, disparities were likely due to different thresholds for admissions and discharge and more importantly of under-reporting of these less serious adverse events in the United States study (Runciman, Webb et al. 2000).

Quantifying the patient safety problem with certainty is thwart with difficulties and many studies have challenged the results of studies attributing patient harm to errors in health care (Aspden, Corrigan et al. 2004). The Harvard Medical Practice Study and subsequent study in Colorado and Utah are observational studies that do not investigate causality and 
are therefore likely to overestimate the problem (McDonald, Weiner et al. 2000). A further study which investigated the results of both United States studies found that although rates of medical error were consistent with their findings, the probability that an error actually caused death was often considered to be low, and the underlying short-term prognosis of the person who died was often judged to be limited (Hayward RA and Hofer TP 2001). Despite the differences in rates of adverse events across studies and discussions regarding the validity of the numbers presented, there is general agreement in the literature that all have highlighted patient safety as a serious issue in health care.

While there is an obvious cost to the patient when subjected to harm during health care, economic costs of medical error are also significant. Several studies have estimated the economic costs related to adverse events (Johnson WG, Brennan TA et al. 1992, Thomas, Studdert et al. 1999, Vincent, Neale et al. 2001, Zhan C and Miller M 2003). Economic costs can be those due to increased health care expenditures (direct costs), and those such as decreased or lost productivity, disability costs, and personal costs of care (indirect costs).

One of the first studies to estimate costs attributable to medical error was conducted by Johnson et al. (1992) for the Harvard Medical Practice Study in 1984. Based on a random sample of 794 admissions with adverse events, the study found total costs of $\$ 878$ million (in 1989 dollars); \$161 million in medical care costs, \$276 million in lost wages, and \$441 million in lost productivity. Subsequently Thomas et al. (1999) reviewed the random sample of 459 adverse events occurring in hospitals in the states of Utah and Colorado and estimated the total costs to be $\$ 661,889,000$ (in 1996 dollars) with direct costs estimated at $\$ 348,081,000$ (Thomas, Studdert et al. 1999). Extrapolation of the results of each of these studies shows state-wide per capita costs of adverse events of \$132 in Utah and Colorado and \$189 in New York State (both adjusted to 1996 dollars). Alternatively Thomas et al. (1999) estimated \$37.6 billion in nation-wide costs of adverse events which equates to approximately $4 \%$ of national health care expenditures.

Other international studies have also found significant economic costs due to medical error. In the United Kingdom, Vincent et al. (2001) concluded that on average each adverse event increased length of stay by 8.5 days (Vincent, Neale et al. 2001). The NHS report, An 
Organisation with a Memory, estimated that each year adverse events result in direct costs of $£ 2$ billion (National Health Service 2000). In Australia, the Quality in Australian Health Care Study concluded an additional 7.1 bed days result from adverse events, equating to eight percent of total hospital bed days, and a cost to the Australian Health Care system \$4.7 billion a year (Wilson RM, Runciman WB et al. 1995).

In New Zealand Davis et al. (1998) found that for each adverse event, length of stay increased, on average, by nine additional days. Based on the results of the New Zealand study, Brown et al. (2002) utilised the prices charged to foreign patients treated by New Zealand hospitals to estimate the health care costs associated with adverse events in New Zealand (Brown, McArthur et al. 2002). The study found that, on average, adverse events resulted in an increase of $\$ 10,264$ per admission. This equates to a total cost of $\$ 870$ million per annum, which in turn suggests that up to $30 \%$ of public hospital expenditures are used to treat adverse events.

\section{Institutional Focus on Quality of Health Care and Patient Safety}

The WHO champions efforts to foster a focus on quality of health care internationally. It argues that quality is a major issue in health care even within developed and well-funded health systems. Therefore, in striving for optimal resource use and maximum coverage, health systems should place quality of care at the forefront of decision making so that optimal results are achieved from health care investment (World Health Organization 2006). The WHO has advanced global awareness in patient safety, recognising patient safety as a "fundamental principle of patient care and a critical component of quality management" (World Health Organization 2006 , pg. 4). In particular, the establishment of the World Alliance for Patient Safety in $\mathbf{2 0 0 4}$ helped to recognise patient safety as an issue of global importance through its focus on patient safety research and the building of a base to enable the goal of achieving safer health care to be met (World Health Organization 2009). In 2006 a collaborative effort between the WHO Collaborating Centre on Patient Safety (Solutions), the World Alliance for Patient Safety, and the Commonwealth Fund was initiated to improve patient safety worldwide (World Health Organization 2006). 
Other examples of institutional recognition of quality of care and patient safety can be found around the world, notably in the United States, United Kingdom, and Australia (National Health Service 2000, AHRQ 2003, ASQHC 2010, National Patient Safety Agency 2011). Agencies within these countries have been established specifically to front and organise the efforts to improve safety and quality through monitoring and identifying patient safety issues. The United Kingdom was one of the first, establishing the National Patient Safety Association (NPSA) ${ }^{3}$, a special health authority of the NHS in 2001. In 2001 the AHRQ Patient Safety Initiative was established in the United States in response to an IOM recommendation ${ }^{4}$ and in 2006, the Australian Commission on Safety and Quality in Health Care (ACSQHC) was established. One of the common goals of each of these organisations is to reduce avoidable patient harm by promoting patient safety throughout the health care system.

There is also much evidence that quality in health and patient safety is a major focus within the New Zealand health sector. The New Zealand Health Strategy ${ }^{5}$ focuses on issues concerning quality and ensuring quality services (Ministry of Health 2000, Ministry of Health 2003). Concerns about the pace of quality improvements led to the establishment in 2010 of the Health Quality and Safety Commission New Zealand (HQSC), responsible for coordinating and leading efforts within the health and disability sector with the purpose of "monitoring and improving the quality and safety of health and disability support services" (Health Quality \& Safety Commission New Zealand 2013, pg. 2).

\subsubsection{Measuring patient safety}

In order to achieve safety (or quality) improvements in health care, there must first be accurate measurement. "We can only be sure to improve what we can actually measure" (Department of Health 2008, pg. 49). Of six integral areas of patient safety research ${ }^{6}$

\footnotetext{
${ }^{3}$ From June 2012 the NPSA patient safety division was transferred to the NHS Commissioning Board Special Health Authority.

${ }^{4}$ See recommendation 4.1 in To Err is Human: Building a Safer Health System (Kohn et al. 2000).

${ }^{5}$ The New Zealand Health Strategy provides the functional framework for the New Zealand health sector and in particular highlights the government's main priorities within the sector.

${ }^{6}$ The other areas of patient safety research which are identified are: understanding the causes, developing solutions, learning from implementation, evaluating impact, and translating improvements into policy and practice. However these are all outside the scope of this research.
} 
identified by the WHO, "measuring the extent of harm caused by health care is the first step towards improving patient safety" (World Health Organization 2009).

The WHO recognises medical record reviews as the "gold standard" in patient safety measurement (World Health Organization 2009). These are undertaken by trained reviewers who retrospectively investigate the paper records of patients for medical error to identify near misses and adverse events. However, while medical record reviews have good reliability and capture a wide range of adverse events (Thomas, Studdert et al. 2000), they are extremely resource intensive and take a long time to complete. This effectively limits them to one off studies and excludes them as a tool for ongoing performance measurement and monitoring. Consequently ongoing monitoring of the patient safety problem will require alternative methods (World Health Organization 2009). Therefore, while the empirical studies discussed have added to the growing awareness and importance of the patient safety problem, the studies are limited in their use to one off pieces of research identifying a problem. As a result, alternative measurement methods must be explored to measure patient safety on a more ongoing basis.

Three alternative methods of measuring adverse events are evident in the literature: incident reporting systems, trigger tools, and statistical indicators. Hospital incident reporting systems require hospital staff involved in patient safety events to report detailed information which is then used to monitor safety issues and for learning and improving patient safety standards. The main purpose of incident reporting systems is to enable health care providers to learn from their experiences (Leape 2002). Reporting systems can involve both mandatory and voluntary reporting requirements and can vary significantly around the world and from hospital to hospital. The IOM's report To Err is Human included the recommendation that hospitals expand their voluntary and in particular mandatory reporting of adverse events (Kohn, J. M.Corrigan et al. 2000).

Incident reporting systems are popular around the world (Beckmann, West et al. 1996, Wu, Pronovost et al. 2002). One example is the National Patient Safety Agency's (NPSA's) National Reporting and Learning System (NRLS) (National Patient Safety Agency 2008). The NRLS leads the world as a nationwide system for the anonymous reporting of health care 
incidents in England. Since 2008 the New Zealand health and disability sector has been guided by a draft national reportable events policy to introduce a national incident management system (Health Quality \& Safety Commission 2012). In 2011 a final version of this policy was developed that makes explicit the requirement for providers to have a process for managing reportable events and is intended to improve quality, safety, and patient experience within the New Zealand health and disability sector. All adverse events and near misses classified as 1, 2, 3, or 4 by the Severity Assessment Code (SAC) ${ }^{7}$ must be reported.

Hospital incident reporting systems have the advantage that they tend to capture a wider picture of patient safety at a lower cost than alternative detection methods, but more importantly by involving front line staff in the process they can establish a culture of patient safety learning and improvement within organisations. On the other hand, research has suggested that the majority of adverse events go unreported (Aspden, Corrigan et al. 2004, Nuckols, Bell et al. 2007). The under-reporting of events is generally attributed to fear of punishment, time demands, and a lack of buy in by its participants (Cullen, Bates et al. 1995). The punitive nature of many incident reporting systems is often cited as the reason their potential has not been reached (Pronovost, Thompson et al. 2005). Consistency and accuracy of reporting also tends to vary within and across organisations. This makes determining and monitoring safety incidents difficult. Furthermore, incident reporting systems capture only the number of safety events (the numerator) but not the number of patients at risk (the denominator). As a result, adverse event rates cannot be calculated so in effect only a small snapshot of the safety issue is captured (AHRQ 2014).

A comparison of the extent to which incident reporting systems are able to capture the adverse events identified in medical record reviews was carried out in a study that reviewed the records of 5,375 patient records across 14 Dutch hospitals. It found that of the 498 adverse events identified by medical record reviews only 10 of those were reported

\footnotetext{
${ }^{7}$ The Severity Assessment Code (SAC) provides guidance on correct follow up procedures as a result of the occurrence of adverse events and near misses based on the consequences of the event and the likelihood with which the event may reoccur. See http://www.apollohealth.co.nz/site/appollo/files//Severity\%20Assessment\%20Code\%20(SAC).pdf
} 
via incident reporting systems (Christiaans-Dingelhoff, Smits et al. 2011). Other studies have formed the same conclusion, that incident reporting systems tend to significantly under-report complaints (Bismark, Brennan et al. 2006, Olsen, Neale et al. 2007, Sari, Sheldon et al. 2007)

Trigger tools are relatively new in patient safety measurement and provide an alternative way of measuring rates of adverse events. They can be viewed as an expedited medical record review whereby trained chart reviewers use a series of prompts to identify potential key adverse events. If a trigger appears in the chart there is a further investigation to determine whether an adverse event actually occurred (Adler, Denham et al. 2008).

In applied research, trigger tools appear to have been originally used as a way of identifying adverse drug events (Classen, Pestotnik et al. 1991). However, their regular use in patient safety measurement is more recent due to the development of the Institute for Healthcare Improvement Global Trigger Tool (IHI GTT). The IHI GTT is increasingly being used in a number of countries around the world including the United States (Good, Saldana et al. 2011, Lau and Litman 2011), the United Kingdom (Franklin, Birch et al. 2010, Haraden and Leitch 2011) and Europe (Doupi, Peltomaa et al. 2013). In New Zealand the Health Round Table and HQSC have been involved in supporting IHI GTT training programmes with a number of DHBs having participated (Health Quality \& Safety Commission 2012). The New Zealand Adverse Drug Event Collaborative (ADEC) has employed the IHI GTT to measure adverse drug events (Seddon, Jackson et al. 2013).

The main advantage of trigger tools is that they provide a relatively efficient and accurate method for measuring and monitoring patient safety. The IHI GTT, which leads the way in this field, has the advantage that it can search relatively large volumes of records, can provide periodic patient safety reports automatically (including rates of adverse events), and can do so in real time (Adler, Denham et al. 2008). However, trigger tools incur significant costs and resource use in order to establish them within hospitals, including specific staff training. Furthermore, they are not automated and still require a chart review to confirm adverse events (Sharek 2012). 
Comparative studies suggest that trigger tools locate the highest proportion of AEs compared with other methods. A United States based study compared the IHI GTT to alternative methods of detecting adverse events. They concluded that the trigger tools found adverse events in one third of hospital admissions, up to ten times higher than the results of the Harvard Medical Practice Study in New York and Utah Colorado (Classen, Resar et al. 2011). One explanation for this discrepancy is that the IHI GTT use broader definitions of adverse events. Others have also suggested that rates will be inflated because the trigger tools capture adverse events associated with temporary harm and events irrespective of preventability (Health Quality \& Safety Commission 2013).

The other approach to measuring quality and safety is the use of statistical indicators which flag adverse events by applying algorithms retrospectively to hospital administrative data. Statistical indicators can be used as a tool to follow trends over time, identify differences between hospitals, and evaluate and prioritise initiatives to reduce patient harm (Rosen, Zhao et al. 2006). Algorithms that define the indicators are applied to hospital administrative data, creating a new field that identifies if a patient was at risk of a given adverse event, and flags whether or not the event was likely to have occurred. 
Table 2: Summary of tools for patient safety (adverse event) measurement

\begin{tabular}{|c|c|c|}
\hline $\begin{array}{l}\text { Harm } \\
\text { Detection } \\
\text { Method } \\
\end{array}$ & Advantages & Limitations \\
\hline \multicolumn{2}{|c|}{ Medical Record Active surveillance can identify } & Substantially underreported harm rates \\
\hline \multirow[t]{5}{*}{ Reviews } & harms not well articulated in chart & \\
\hline & Measures "all cause" harm & $\begin{array}{l}\text { Relies partially on voluntary or verbally solicited } \\
\text { identification of harm }\end{array}$ \\
\hline & $\begin{array}{l}\text { Provides a rate (i.e., harms per } \\
100 \text { admissions) }\end{array}$ & Active real time surveillance is resource intensive \\
\hline & & $\begin{array}{l}\text { Unfocussed review of charts is also resource } \\
\text { intensive }\end{array}$ \\
\hline & & $\begin{array}{l}\text { Retrospective review of charts challenging if } \\
\text { poor/incomplete documentation }\end{array}$ \\
\hline Incident & Well established process in most & Identifies only between $2 \%$ and $8 \%$ of harmful \\
\hline \multirow[t]{4}{*}{ Systems } & Inexpensive & Focus tends to be on error, not harm \\
\hline & Easy information to obtain & Voluntary nature results in vast underreporting \\
\hline & & Can be time intensive \\
\hline & & Often perceived as punitive by staff \\
\hline \multirow[t]{8}{*}{ Trigger Tools } & Measures "all cause" harm & Requires training \\
\hline & Measures total harm burden & $\begin{array}{l}\text { Resource intensive: } \mathrm{IHI} \text { recommends } 20 \text { charts per } \\
\text { month at } 20 \text { minutes per chart }\end{array}$ \\
\hline & $\begin{array}{l}\text { Provides a rate (i.e., harms per } \\
100 \text { admissions) }\end{array}$ & Global trigger tools not automated \\
\hline & $\begin{array}{l}\text { Focuses on harm, but includes } \\
\text { errors as well }\end{array}$ & Retrospective review \\
\hline & Allows sampling strategy & $\begin{array}{l}\text { Retrospective review of charts challenging if } \\
\text { poor/incomplete documentation }\end{array}$ \\
\hline & $\begin{array}{l}\text { Relatively efficient: } 20 \text { minutes } \\
\text { per chart }\end{array}$ & \\
\hline & Can be population specific & \\
\hline & $\begin{array}{l}\text { Excellent specificity and very good } \\
\text { sensitivity }\end{array}$ & \\
\hline Statistical & Standard definitions & Identifies less than $10 \%$ of all harms \\
\hline $\begin{array}{l}\text { Indicators } \\
\text { based on }\end{array}$ & $\begin{array}{l}\text { Method allows direct comparison } \\
\text { between hospitals }\end{array}$ & Poor sensitivity and specificity \\
\hline \multirow[t]{3}{*}{$\begin{array}{l}\text { Administrative } \\
\text { Data }\end{array}$} & Inexpensive to obtain data & $\begin{array}{l}\text { Focus is on only a few specific harm types (not "all } \\
\text { cause" harm) }\end{array}$ \\
\hline & & $\begin{array}{l}\text { Harm easily hidden/missed (if not well described } \\
\text { in charting) }\end{array}$ \\
\hline & & Dependent on accuracy of chart coding \\
\hline
\end{tabular}

Source from (Sharek 2012)

The main advantage of statistical indicators is that they are used with hospital administrative data. These data are generally used for administering health care delivery, enrolling members into health plans, and reimbursing for services (lezzoni 1997). In New 
Zealand the National Minimum Dataset (NMDS) is a national collection of all people admitted to publicly funded hospitals and publicly funded events at private hospitals (National Health Board Business Unit 2010). Administrative data are readily available (free or inexpensive to use, and available in a timely manner), and are typically all-inclusive (include admissions across entire populations) (Iezzoni 1997). Statistical indicators are particularly appealing, as they too possess these same advantageous qualities. Their use with administrative data also means statistical indicators are unobtrusive (do not rely on individuals reporting the details of adverse events they have witnessed or been involved in), can be automated, easily implemented periodically, and can cover a range of adverse events.

The major concern with use of administrative data is the limited clinical detail they include (Zhan C and Miller MR 2003). As a result, statistical indicators can have low sensitivity and specificity $^{8}$ (Bates, O'Neil et al. 1995, West, Weeks et al. 2008). However, Romano et al demonstrated that concentrating on specific adverse events for a specific population can improve specificity significantly (Romano P, Chan B et al. 2002).

Zhan and Miller (2003) provide a detailed review of statistical indicators based on administrative data (Zhan C and Miller MR 2003). At the time they found statistical indicators had been scarcely employed in research on quality of care and patient safety. They identified three early studies from the 1990s which advocated the use of indictors with claims data to identify adverse events. The first encouraged the use of indicators to assist in guiding medical record reviews (Roos and Brazauskas 1990). The second, a programme established at the United Healthcare Corporation, suggested using indicators to explore the incidence of adverse events in addition to other outcome measures (Leatherman, Peterson et al. 1991). The last used Medicare data to detect post-operative adverse events which resulted in subsequent patient readmissions (Riley, Lubitz et al. 1993).

\footnotetext{
${ }^{8}$ Sensitivity is the fraction of positive outcomes correctly predicted and specificity is the fraction of negative outcomes correctly predicted.
} 
Zhan and Miller recognise lezzoni's research in the early 1990s as the first systematic investigation of quality and safety indicators (lezzoni LI, Foley SM et al. 1992). This research, known as the Complications Screening Programme (CSP) culminated in a list of 27 indicators of potentially preventable complications. In the mid-1990s, in response to the growing need for accessible and reliable health care quality indicators, the AHRQ who had previously supported the earlier work of lezzoni developed a set of 33 quality indicators known as the Healthcare Cost and Utilization Project ${ }^{9}$ (HCUP).

As the knowledge base of safety measurement and patient safety indicators increased, the AHRQ funded a major project in the late 1990s to build and improve on the CSP and HCUP QIs (AHRQ 2007). In partnership with the University of California San Francisco-Stanford Evidence Based Practice Centre (UCSF-Stanford EPC), four modules of quality indicators were developed, one of which is the AHRQ PSIs.

The OECD Health Care Quality Indicators (HCQI) Project was launched in 2003 to implement quality measures for international benchmarking of medical care at the health system level (Mattke, Epstein et al. 2006). The project recommended a list of 21 indicators for patient safety developed from an initial candidate list of 59 indicators. The candidate list included the AHRQ PSIs, the Australian Council for Safety and Quality Indicators, Complications Screening Programme Beth Israel Hospital (BIH) Indicators, Joint Commission on Accreditation of Healthcare Organisations (JCAHO) Indicator Measurement (IM) System infection Control Indicators, and the JCAHO sentinel events (McLoughlin, Millar et al. 2006).

More recently the Safety Improvement for Patients in Europe (SIMPATIE) project was established to develop a set of safety indicators to be used for improvements of safety in health care in Europe. The project developed a set of indicators utilising the AHRQ PSIs, indicators from the OECD HCQI project, and indicators from the Danish National Indicator Project (DNIP) (Kristensen, Mainz et al. 2007).

\footnotetext{
${ }^{9}$ The Healthcare Cost and Utilization Project is developed by a combination of Federal, State, and health industry participants and sponsored by the AHRQ. HCUP consists of health care databases, products, and software tools. See http://www.hcup-us.ahrq.gov/overview.jsp for more details.
} 
Interestingly few national initiatives exist which have independently developed quality or safety indicators. The majority of the literature on indicator development seems to have been developed by institutes or via the collaboration of institutes which have searched for candidate lists of indicators, some recognised some not, and carried out their own research to refine these lists and create their own indicator set. What is evident from such studies is that the AHRQ PSIs form a major part of these projects. This is echoed by Tsang et al. who reviewed the literature on patient safety measures derived from routinely collected hospital data to inform indicator development. They found more than two thirds of the articles reviewed used the AHRQ PSIs (Tsang, Aylin et al. 2008).

\section{The Agency for Healthcare Research and Quality (AHRQ) Patient Safety Indicators (PSIs)}

The AHRQ PSIs are among the most recognised statistical indicators for adverse events (Tsang, Aylin et al. 2008). The AHRQ PSIs consist of 20 provider-level and seven area-level PSIs and were initially released in 2003 (See Table 3 for complete list of the 20 PSIs). They are one of four modules of Quality Indicators (QIs) developed by the $A H R Q^{10}$. Their purpose is to help identify potentially preventable complications and iatrogenic events for patients treated at hospitals and become a starting point for analysis to help reduce such errors through system or process changes (AHRQ 2007). This section presents the history and development of the AHRQ PSIs, discusses their subsequent evaluation, and summarises their applied use in health care settings and health care research in general.

\section{History of PSI Development and Steps in their Creation and Evaluation}

As discussed, the AHRQ developed a set of 33 quality indicators known as the HCUP QIs in the 1990s in response to the growing need for accessible and reliable health care quality indicators (AHRQ 2007). These AHRQ indicators were developed so that they required only the information typically found in hospital administrative data. Over time, as the knowledge base of safety measurement and patient safety indicators increased, the AHRQ funded a

\footnotetext{
${ }^{10}$ The remaining three QIs developed by AHRQ are: the prevention quality indicators which indicate those hospital admissions which could have been avoided if adequate outpatient care had been provided; the inpatient quality indicators, designed to reflect quality of hospital care through mortality rates, underuse, overuse and misuse of care; and the paediatric quality indicators, a composite set derived from the first three modules of QIs to focus specifically on the quality of care received by children.
} 
major project to build and improve on the HCUP QIs. This project was conducted by the University of California San Francisco-Stanford Evidence Based Practice Centre (UCSFStanford EPC), which developed the four modules of Qls discussed previously, one of which is the AHRQ PSIs.

During their development the PSIs were subjected to a rigorous five-stage evaluation procedure. This began with developing a conceptual framework within which the scope of the project could be defined, an evaluation framework was constructed, and standardised definitions of key terms established. Stage two included a thorough review of the literature to ascertain a list of possible PSIs. In stage three a candidate list of PSIs was established which was then reviewed by panels to test for face validity ${ }^{11}$ and experts in clinical coding to see if the intended complications/adverse events and the populations at risk were in fact captured by the PSIs as desired. Stage four included several stages of indicator assessments: whether the indicator measures a complication and not something present on admission; how preventable the complication is; the degree of medical error in causing the complication; how likely a complication is recorded in the patient medical chart given that it actually occurs; and to what degree casemix (such as patient age) affects the indicator. Finally the PSIs undertook an empirical evaluation, specifically investigating observed hospital level indicator rates compared to hospital level rates adjusted for casemix (bias ${ }^{12}$ ) and rates adjusted for reliability (precision ${ }^{13}$ ) (McDonald, Romano et al. 2002, AHRQ 2007).

\footnotetext{
${ }^{11}$ Face validity ensures that indicators reflect a feature of hospital safety generally regarded as important and that hospitals have some degree of control over.

${ }^{12}$ Bias investigates the effect on the indicator of variations in patient demographics and clinical characteristics of admissions, and the possibility to apply risk adjustment and statistical methods to remove most or all bias.

${ }^{13}$ Precision requires there be a substantial amount of hospital-level variation that is not attributable to random variation.
} 
Table 3: The AHRQ PSIs

\begin{tabular}{|c|c|}
\hline PSI & Description \\
\hline $\begin{array}{l}\text { PSI1 } \\
\text { general }\end{array}$ & $\begin{array}{l}\text { Complications of anaesthesia - Cases of anaesthetic overdose, reaction, or endotracheal tube } \\
\text { misplacement for surgery discharges. Excludes codes for drug use and self-inflicted injury. }\end{array}$ \\
\hline $\begin{array}{l}\text { PSI2 } \\
\text { medical }\end{array}$ & $\begin{array}{l}\text { Death in low mortality DRGs - In-hospital patient death in DRGs with less than } 0.5 \% \text { mortality. } \\
\text { Excludes trauma, immuno-compromised, and cancer patients. }\end{array}$ \\
\hline $\begin{array}{l}\text { PSI3 } \\
\text { medical }\end{array}$ & $\begin{array}{l}\text { Decubitus ulcers - Cases of decubitus ulcer for discharges with a length of stay of } 5 \text { or more days. } \\
\text { Excludes patients with paralysis or in MDC } 9 \text { (Skin, subcutaneous tissue and breast), MDC } 14 \\
\text { (Pregnancy, childbirth and puerperium), and patients admitted from a long-term care facility. }\end{array}$ \\
\hline $\begin{array}{l}\text { PSI4 } \\
\text { medical }\end{array}$ & $\begin{array}{l}\text { Failure to rescue - Death of patient having developed specified complications of care during } \\
\text { hospitalization. Excludes patients age } 75 \text { and older, neonates in MDC } 15 \text { (Newborns and other } \\
\text { neonates), patients admitted from long-term care facility and patients transferred to or from } \\
\text { other acute care facility. }\end{array}$ \\
\hline $\begin{array}{l}\text { PSI5 } \\
\text { general }\end{array}$ & $\begin{array}{l}\text { Foreign body left during procedure - Discharges with foreign body accidentally left in during } \\
\text { procedure. }\end{array}$ \\
\hline $\begin{array}{l}\text { PSI6 } \\
\text { general }\end{array}$ & $\begin{array}{l}\text { latrogenic pneumothorax - Cases of iatrogenic pneumothorax. Excludes trauma, thoracic surgery, } \\
\text { lung or pleural biopsy, or cardiac surgery patients, and MDC } 14 .\end{array}$ \\
\hline $\begin{array}{l}\text { PSI7 } \\
\text { medical }\end{array}$ & $\begin{array}{l}\text { Selected infections due to medical care - Episodes with ICD-10-AM diagnosis code of: Infections } \\
\text { following infusion transfusion \& therapeutic injection, Infection and inflammatory reaction due to } \\
\text { other cardiac and vascular devices, implants and grafts and infection following immunisation. } \\
\text { Excludes patients with immune-compromised state or cancer. }\end{array}$ \\
\hline $\begin{array}{l}\text { PSI8 } \\
\text { general }\end{array}$ & $\begin{array}{l}\text { Postoperative hip fracture - Cases of in-hospital hip fracture for surgical discharge. Excludes } \\
\text { patients in MDC } 8 \text { (Musculoskeletal system and connective tissue), with conditions suggesting } \\
\text { fracture present on admission and MDC } 14 \text {. }\end{array}$ \\
\hline $\begin{array}{l}\text { PSI9 } \\
\text { surgical }\end{array}$ & $\begin{array}{l}\text { Postoperative haemorrhage or haematoma- Cases of hematoma or haemorrhage requiring a } \\
\text { procedure. Excludes MDC } 14 .\end{array}$ \\
\hline $\begin{array}{l}\text { PSI10 } \\
\text { surgical }\end{array}$ & $\begin{array}{l}\text { Postoperative physiologic and metabolic derangement - Cases of specified physiological or } \\
\text { metabolic derangement for surgical discharges. Excludes patients with principal diagnosis of } \\
\text { diabetes, with diagnoses suggesting increased susceptibility to derangement and obstetric } \\
\text { admissions. }\end{array}$ \\
\hline $\begin{array}{l}\text { PSI11 } \\
\text { surgical }\end{array}$ & $\begin{array}{l}\text { Postoperative respiratory failure - Cases of acute respiratory failure. Excludes MDC } 4 \text { (Respiratory } \\
\text { system) and MDC } 5 \text { (Circulatory system) and obstetric admissions. }\end{array}$ \\
\hline $\begin{array}{l}\text { PSI12 } \\
\text { surgical }\end{array}$ & $\begin{array}{l}\text { Postoperative pulmonary embolism or DVT - Cases of deep vein thrombosis or pulmonary } \\
\text { embolism for surgical discharges. Excludes obstetric patients. }\end{array}$ \\
\hline $\begin{array}{l}\text { PSI13 } \\
\text { surgical }\end{array}$ & $\begin{array}{l}\text { Postoperative sepsis - Cases of sepsis for elective surgery patients, with length of stay more than } 3 \\
\text { days. Excludes principal diagnosis of infection, or any diagnosis of immune-compromised state or } \\
\text { cancer, and obstetric admissions. }\end{array}$ \\
\hline $\begin{array}{l}\text { PSI14 } \\
\text { surgical }\end{array}$ & $\begin{array}{l}\text { Postoperative wound dehiscence - Cases of reclosure of postoperative disruption of abdominal } \\
\text { wall during abdominopelvic surgery. Excludes obstetric admissions. }\end{array}$ \\
\hline $\begin{array}{l}\text { PSI15 } \\
\text { general }\end{array}$ & $\begin{array}{l}\text { Accidental puncture or laceration - Cases of technical difficulty (e.g., accidental cut or laceration } \\
\text { during procedure). Excludes obstetric admissions. }\end{array}$ \\
\hline $\begin{array}{l}\text { PSI16 } \\
\text { general }\end{array}$ & Transfusion reaction - Cases of transfusion reaction \\
\hline $\begin{array}{l}\text { PSI17 } \\
\text { obstetric }\end{array}$ & $\begin{array}{l}\text { Birth trauma, injury to neonate - Cases of birth trauma, injury to neonate. Excludes some preterm } \\
\text { infants and infants with oestrogenic imperfecta. }\end{array}$ \\
\hline $\begin{array}{l}\text { PSI18 } \\
\text { obstetric }\end{array}$ & $\begin{array}{l}\text { Obstetric trauma, vaginal delivery with instrument - Cases of obstetric trauma (3rd or 4th degree } \\
\text { lacerations) during instrument-assisted vaginal deliveries. }\end{array}$ \\
\hline $\begin{array}{l}\text { PSI19 } \\
\text { obstetric }\end{array}$ & $\begin{array}{l}\text { Obstetric trauma, vaginal delivery without instrument - Cases of obstetric trauma (3rd or 4th } \\
\text { degree lacerations) during vaginal deliveries without instrument assistance. }\end{array}$ \\
\hline $\begin{array}{l}\text { PSI20 } \\
\text { obstetric }\end{array}$ & $\begin{array}{l}\text { Obstetric trauma, caesarean delivery - Cases of obstetric trauma (3rd or 4th degree lacerations) } \\
\text { during caesarean deliveries. }\end{array}$ \\
\hline
\end{tabular}


The AHRQ PSIs have subsequently been revised for use with Australian administrative data and are known as the AusPSIs (Department of Health and Human Services 2009). The revision is required because the AHRQ PSIs use a version of ICD coding (ICD-9-CM) which is no longer used in Australia. As a result ICD-9-CM codes have been translated to the ICD version standard in Australia (ICD-10-AM). These translations have made it possible for the AHRQ PSIs to be used with New Zealand administrative data since New Zealand also uses ICD-10-AM coding. As part of the Enhancing Hospital Outcomes Project ${ }^{14}$ these algorithms have been revised and then applied to nine years of NMDS from 2001 to $2009^{15}$.

\section{Further Evaluation on AHRQ PSIs}

Several independent studies have since shown the PSIs to have good face and construct validity based on United States data ${ }^{16}$ (Zhan C and Miller MR 2003, Rosen, Rivard et al. 2005, Rosen, Zhao et al. 2006). However, subsequent studies have also demonstrated concerns regarding validity of some of the PSIs based on poor positive predictive value (PPV) (Cevasco, Borzecki et al. 2011, Chen, Rosen et al. 2011, Kaafarani, Borzecki et al. 2011). A similar study investigated 12 PSIs and found moderate PPV for many and concluded the use for the PSIs may be best restricted to screening and case-finding until improvements are made through coding revisions such as the inclusion of present on admission ${ }^{17}$ (POA) flags (Rosen, Itani et al. 2012).

While validation of the AHRQ PSIs has largely been based on United States data, validation based on populations outside of the United States has increasingly been undertaken. This includes in the United Kingdom, Germany, Canada, and Australia where comparable adverse event rates have been found (Raleigh, Cooper et al. 2008, Bottle and Aylin 2009).

\footnotetext{
${ }^{14}$ The Enhancing Hospital Outcomes project was designed to analyse the quality of patient care in New Zealand using indicators to measure a variety of health conditions and events.

${ }^{15}$ Modifications were made to PSI2: Death in Low Mortality DRGs to be consistent with New Zealand data and PSI7: Select Infections Due to Medicare Modifications where minor modifications were made to secondary diagnosis coding.

${ }^{16}$ Construct validity requires the indicator to perform well in identifying true (or actual) quality of care problems.

${ }^{17}$ Present on admission flags are additional variables in administrative data which identify whether a condition was present on admission or if it may have occurred during a hospital stay.
} 
Evaluation work in New Zealand has also been recently completed (Hider, Parker et al. 2014). At the time of writing it was known that the work of Hider and colleagues was ongoing although the details of the work were not known. Their aim was to apply 16 of the AHRQ PSIs to New Zealand data and concludes that not only was the application successful, but several of the indicators could be used to monitor adverse events in New Zealand hospitals to aid in quality improvement. Rates of adverse events were calculated for each indicator. Variation over time was assessed for three of the PSIs ${ }^{18}$ and risk adjustment was applied to the same three indicators to examine variation across hospitals. The study identified 99,366 admissions flagged with an adverse event, however a rate of adverse events cannot be inferred as the study does not identify the relevant denominator. A number of indicators with low rates of adverse events (less than one percent) were highlighted. Based on the three indicators investigated in detail the study found considerable variation in both rates over time and across hospitals.

Hider et al. do not cover the obstetrics indicators. Furthermore, in depth investigation of intertemporal and cross-sectional variation is limited to just three indicators. Risk adjustment is conducted on only three indicators and there is no evaluation of the effect of risk adjustment on hospital level rates.

\section{Applied use of the AHRQ PSIs}

In the United States the National Quality Forum (NQF) endorsed 10 PSIs as consensus standards in 2008 (National Quality Forum 2008) and the Centers for Medicare and Medicaid Services (CMS) publically report on six PSIs and a PSI composite measure on their Hospital Compare website (Centers for Medicare and Medicaid Services). Currently the AHRQ PSIs are being explored for use in health care settings in New Zealand by the HQSC.

The AHRQ PSIs have been used in applied research settings in the United States: PSIs were used to investigate the impact of patient safety events on mortality rates, length of stay, and treatment cost (Zhan C and Miller M 2003, Rivard, Luther et al. 2008); Coffey et al

\footnotetext{
${ }^{18}$ The three PSIs analysed in more detail were: PSI4, Failure to rescue; PSI9, Postoperative haemorrhage; PSI12, Postoperative DVT/PE.
} 
(2005) used the AHRQ PSIs to investigate whether adverse event rates differ between racial and ethnic groups after controlling for socio economic status (Coffey, Andrews et al. 2005); the effects of reforms on resident duty hours were analysed by investigating the change in PSI rates (Rosen, Loveland et al. 2009); Rivard et al (2006) investigated the association between patient safety and hospital teaching status (Rivard P, Christiansen C et al. 2006); and Carey and Stefos (2011) explored the use of 15 PSIs as measures of hospital quality and the estimation of a hospital cost function to examine the relationship between cost and quality of care (Carey and Stefos 2011).

Compared to alternative measurement tools the AHRQ PSIs have several limitations and there are especially concerns about their accuracy in flagging true safety events. Despite these shortcomings they enjoy several strengths that are not matched by alternative methods: they are inexpensive to use, comprehensive, unobtrusive, and can be used periodically. For these reasons their use warrants further exploration in the New Zealand setting and they form the basis for hospital patient safety and quality measurement for this research.

\subsection{Risk Adjustment}

\subsubsection{Introduction to Risk Adjustment}

Hospitals differ in terms of the patient populations (or casemix) they serve cross-sectionally and inter-temporally. Casemix variation can be due to patient demographics such as age, gender, and ethnicity as well as clinical factors such as the conditions and degree of severity patients present with or the comorbidities they have. Older patients for example, or those who present with greater severity of illness will, on average, be expected to develop more complications and experience worse health outcomes. In comparing hospital outcomes, risk adjustment is an essential process that attempts to control for dissimilar casemix. Its purpose is to enable fairer and more valid comparisons between different providers and over time.

It is widely accepted that meaningful comparisons of quality of care must incorporate adjustments for differences in casemix (DeLong, Peterson et al. 1997, lezzoni 2003). "Valid 
conclusions regarding the differences in quality among providers require the removal of the confounding effect of different institutions providing care to patients with dissimilar severity of illness and case complexity" (Wray N, Hollingsworth J et al. 1997, pg. 327). Donabedian's rubric for quality of care stresses the possible causal relationship between outcomes and quality of care, highlighting the importance of risk adjustment because factors other than quality may influence outcomes (Donabedian 1966). Without it, incorrect conclusions about quality of care can easily be drawn.

\subsubsection{Applied Risk Adjustment}

Systematic risk adjustment in health settings was first employed within the health insurance sector to set payments for plans and ensure those payments fairly reflect the expected costs of health care provision (Schone and Brown 2013). Some Medicaid programmes began using risk adjustment as early as the 1990s including Maryland in 1997 and Colorado and Oregon in 1998 (Martin, Rogal et al. 2004). Medicare began funding risk adjustment approaches in the early and mid-1990s (Pope, Adamache et al. 1998).The Affordable Care Act (ACA) enacted by President Obama in 2010 requires risk adjustment to ensure health insurance providers do not benefit from enrolling a disproportionate number of healthy individuals. Risk adjustment has also been employed in countries such as Canada, Netherlands, and Germany to assist in allocating funds to providers of health care in an equitable manner (Lu, Moores et al. 2002, Buchner and Wasem 2003, Van de Ven, Beck et al. 2003, Van de Ven, van Vliet et al. 2004).

Risk adjustment is increasingly being used for non-payment purposes. These include controlling for quality, detecting performance improvements, and ranking and rating providers (McKillop, Pink et al. 2001, Murgolo 2002, Shwartz, Ash et al. 2005). One of the most well-known uses of risk adjusted quality measures is the New York Cardiac Surgery Reporting System's risk adjusted mortality rates for coronary artery bypass grafting (CABG) and heart valve surgery (Jha and Epstein 2006). The Veteran's Health Administration (VA) produces risk adjusted outcomes data on specific surgical interventions through the National VA Surgical Quality Improvement Program (NSQIP) (Fuchshuber, Greif et al. 2012). The Center for Continuous Quality Improvement in Cardiac 
Surgery uses risk adjusted outcomes among cardiac surgery patients (Health Services Research and Development Service 1997). More recently the AHRQ has produced four modules of risk adjusted quality measures, the prevention quality indicators, inpatient quality indicators, paediatric quality indicators, and the patient safety indicators (AHRQ 2007). In New Zealand the MoH employs risk adjustment to construct age standardised mortality in their annual publication on mortality (Ministry of Health 2015). The HQSC New Zealand publishes a range of quality and safety indicators many of which are adjusted for gender, age, ethnicity, and other relevant risk factors (Health Quality \& Safety Commission New Zealand 2013).

\subsubsection{Methods for Risk Adjustment see}

A common method for estimating risk adjusted rates is direct standardisation (DS). Hospital specific rates are calculated for each risk stratum and applied to the standard reference population. This produces the rate a hospital would have, if it had the casemix of the reference population. The major criticism of DS is that the sample sizes within some strata can be too small to produce reliable results. DS is conceptually appealing but has practical difficulties. As a result DS is rarely used when risk adjustment is intended to control for multiple confounders or to profile hospitals (lezzoni 2003).

An alternative is indirect standardisation. This applies stratum-specific rates observed in the reference population to hospital populations of interest to calculate expected rates. In other words, the derived result can be interpreted as the rate a hospital would be expected to have, should it perform in line with the reference population. Comparisons of observed to expected rates are then undertaken (DeLong, Peterson et al. 1997, lezzoni 2003). A significant concern with indirect standardisation is that a hospital which has comparatively worse observed rates in each stratum, but a more favourable casemix, can be shown to be a comparatively better performer (lezzoni 2003). However, when the number of risk factors are small, for example when adjusting for age only, indirect standardisation is often used in practice. Breslow and Day employ this approach of risk adjustment to control for age in the comparison of mortality and morbidity across 
populations (Breslow and Day 1975). Indirect standardisation was also used to control for age in patients who experienced with uterovaginal prolapse (Mant, Painter et al. 1997).

For dichotomous outcomes logistic regression models, the generalisation of indirect standardisation, is typically applied and this is the approach that will be used in this study. Multivariate regression modelling is the most common method for risk adjustment (DeLong, Peterson et al. 1997, Shahian, Normand et al. 2001, lezzoni 2003). At the event level, the outcome of interest is regressed on the full set of risk factors to estimate risk adjustment coefficients using all at-risk admissions. The estimated coefficients from these regressions are used to derive predicted probabilities of the outcome of interest for each at-risk patient. These predicted probabilities can then be summed to derive hospital level expected rates.

Logistic regression based risk adjustment is standard in most performance profiling and applied use is widespread in the literature. It is the approach taken by the AHRQ for their risk adjustment of the PSIs (AHRQ 2011). It is also the method used by the risk adjustment American College of Surgeons National Surgical Quality Improvement Program (Cohen, Dimick et al. 2009). Dimick and Osbourne used logistic regression based risk adjustment to compare the quality of hospital surgical units (Dimick, Osborne et al. 2010).

The major advantage of multivariate regression modelling relative to the previous methods discussed is that it permits the use of a large number of risk factors. Furthermore, statistical software is available for estimating logistic regression models even when sample sizes are large and risk factors are numerous. It is also the standard method in health care, and is subsequently a method of outcome based quality comparison that is widely understood and accepted within the sector (Shaughnessy and Hittle 2002). On the other hand logistic based risk adjustment methods for quality comparisons have received criticism (Shahian, Normand et al. 2001). Logistic regression with rare events can suffer from small-sample bias (King and Zeng 2001). The degree of bias is dependent on the frequency of the outcome of interest and sample size: infrequent events with small sample size will have substantial bias however a sufficiently large sample size will alleviate the problem. Other criticisms include inherent imprecision, which is compounded when the results of patient-level models are 
aggregated to assess provider performance. Issues of sample size differences, clustering of observations, multiple comparisons, and failure to account for the random component of inter-provider variability.

Other methods have been used but they generally have disadvantages. Perhaps the simplest alternative is restriction. This method excludes certain patients from the analysis, leaving the remaining patients more comparable (Joint Commission Resources 2011). A danger of the method is it can result in small sample sizes. Furthermore, there is a limit to the range of risk factors that can be accounted for. Stratification has also been employed as an alternative to calculating expected rates. This is the process of dividing patients into a number of separate groups based on risk factors deemed to be confounders for the outcome of interest. Outcomes are then analysed independently within stratum. However analysis is often limited to controlling for one risk factor at a time; at best only a few risk factors can be accounted for without either generating concerns of sample size and/or creating excessive numbers of stratum to analyse.

More recently, researchers have advocated the use of alternative, more sophisticated statistical models to address some of these concerns (Shahian, Normand et al. 2001). Some researchers have used multilevel (or hierarchical) models to account for non-random clustering of patients within providers (Gatsonis, Normand et al. 1993, Christiansen C and Morris C 1997, Shahian, Normand et al. 2001). Thomas et al (1994) used an empirical Bayes model to account for the different levels of variation in provider quality measures, producing more accurate results (Thomas, Longford et al. 1994). The AHRQ have also employed more sophisticated methods for adjustment, particularly to account for noise.

\subsubsection{Empirical Evaluation of Risk Adjustment Models}

Key statistical performance measures of risk adjustment models can be employed to determine both the overall model performance and assist in variable selection. Discrimination and calibration measures are typically used to assess risk adjustment models empirically (DeLong, Peterson et al. 1997, Harrell 2001). In addition, "global" measures, combining both discrimination and calibration such as likelihood ratio tests and $R^{2}$ values 
can also be considered (Harrell 2001). It is important to realise that no single summary indicator of a model's statistical performance is sufficient and several measures are often used concurrently (lezzoni 2003).

Discrimination is the extent to which a model predicts higher probabilities of an outcome occurring for those patients who actually experience the outcome than for those who do not. A variety of discrimination measures exist (Pencina, D'Agostino et al. 2008). For dichotomous outcomes, a starting point is to create pairwise combinations of predicted and observed outcomes based on predefined cut-off levels from which key performance measures can be derived: sensitivity, specificity, predicted positive value, and predicted negative value. An issue with such measures is that they are all dependent on the cut-off value. lezonni describes choosing a cut-off as situation specific, and states that no single cut-off is obviously best (lezzoni 2003).

The c-statistic is the most common statistical performance measure to assess models of dichotomous outcomes and is employed in this study (Pencina, D'Agostino et al. 2008). It is defined as the area under the receiver operating characteristic (ROC) curve (the curve of sensitivity versus (1-specificity) across all prediction cut-off values) (Hanley and McNeil 1982). Its major appeal is that it is independent of the cut-off value and combines both sensitivity and specificity measures. It has been used for comparing mortality models, predicting outcomes, and evaluating the explanatory power of additional risk factors (Knaus, Wagner et al. 1991, Krakauer, Bailey et al. 1992, Hannan, Kilburn et al. 1994, Khuri, Daley et al. 1997). The c-statistic is also reported by the AHRQ in the development of their PSI risk adjustment models (AHRQ 2011). However, when assessing the value of including additional risk factors, it has been criticised as being less sensitive than alternative, "global" measures of fit (Harrell 2001).

Harrell argues that more sensitive measures such as the likelihood ratio test should also be employed to assess the inclusion of additional variables (Harrell 2001). The likelihood ratio test is used to compare the fit of alternative models, one of which is nested within the other. The test is based on the likelihood ratio, which expresses how many times more likely the data are under one model than the other. The null hypothesis for the test is that 
the nested model is true. The likelihood ratio test is widely used in developing and evaluating risk adjustment models. Certow et al. employ likelihood ratio tests as a means of comparing alternative risk adjustment models for death after acute renal failure (Chertow, Soroko et al. 2006). Klabunde et al. use the likelihood ratio test to compare nested models to create a comorbidity index using Medicare data (Klabunde, Potosky et al. 2000). The likelihood ratio test is used by Jenkins et al to assess whether the inclusion of additional clinical risk factors was more predictive of in-hospital mortality for children younger than 18 after congenital heart disease surgery (Jenkins, Gauvreau et al. 2002). 


\section{Section 3 Methods}

The remainder of the thesis is built around the application of the AHRQ PSIs to New Zealand administrative data. Section 3.1 provides some background information to the AHRQ PSIs and describes their application to administrative data, specifically NMDS. Section 3.2 discusses the background to risk adjustment in more detail. Section 3.3 summarises the methods employed to develop risk adjustment models, defining the incremental models, and discussing additional variable selection criteria. The estimation methods for risk adjustment are discussed in Section 3.4, including how a brief comparison to alternative estimation methods is made. Finally, the empirical methods employed to evaluate the risk adjustment models are summarised in Section 3.6.

\subsection{AHRQ PSIs}

The purpose of the PSIs is to help identify potentially preventable complications and iatrogenic (caused by medical examination or treatment) events for patients treated at hospitals and they become a starting point for analysis to help reduce such errors (AHRQ 2007). In other words they "screen for problems that patients experience as a result of exposure to the healthcare system that are likely amenable to prevention by changes at the system or provider level" (AHRQ 2007, pg. 2). Each of the 20 PSIs focuses on separate predefined potential AEs and areas of patient safety, and all reflect in various ways the multidimensional concept of hospital safety and quality.

The PSIs work by applying algorithms to hospital administrative data. These algorithms screen each discharge level observation to identify amongst other things whether specific diagnosis codes are present. For each PSI a new field is created for each observation that flags whether or not an AE potentially occurred, based on the clinical coding of the individual patient discharge.

There are two key components to each PSI algorithm: the denominator, and the numerator. The denominator defines the group of patients who are considered at risk of experiencing the AE. The numerator defines the group of patients who are considered to have 
experienced the AE. Definitions for each PSI differ, incorporating a combination of diagnosis and procedure codes, and demographic information such as gender and patient age.

The denominator for each PSI is typically defined by broad groups of patients such as "all medical discharges", "all surgical discharges", or "all episodes". However most PSIs contain certain denominator inclusions and exclusions as well. For example, PSIs (with some exceptions) exclude patients younger than 18 years from the denominator. Further exclusions exist when specific clinical codes arise in either primary or secondary diagnoses, if episodes are categorised by specific Diagnostic Related Groups (DRGs) and Major Diagnostic Categories (MDCs), or based on a minimum duration of hospital stay. The numerator of each PSI identifies events where a "secondary diagnosis code flags a potentially preventable complication" (AHRQ 2007, pg. 2).

Box 1 illustrates how a PSI is defined and is taken from the AusPSI's technical specifications. For a detailed non-technical description of each PSI, the AEs they identify, how these events can occur, how they can be prevented, and hence why the PSIs help to quantify hospital safety and quality, see Appendix A.

\section{Box 1: Complications with Anaesthesia (PSI1)}

\section{Numerator:}

Episodes with ICD-10-AM diagnosis code for complication with anaesthesia in any secondary diagnosis field.

\section{Denominator:}

All surgical episodes, 18 years and older or MDC 14 (Pregnancy, Childbirth, and Puerperium), defined by an ICD-10-AM procedure code for an operating room procedure or anaesthetic.

\section{Exclude:}

Episodes with an ICD-10-AM diagnosis code for complication of anaesthesia in the principal diagnosis field.

Episodes with codes for self-inflicted injury, poisoning due to anaesthetics and an ICD-10AM diagnosis code for active drug dependence or active nondependent abuse of drugs. 


\subsubsection{Application of the AHRQ PSIs to NMDS}

The AHRQ PSI algorithms were first modified and revised for use with ICD-10-AM by Victoria Healthcare and subsequently by Compass Research Centre at Auckland University for use with NMDS. The algorithms applied to NMDS generate 20 PSI indicator variables at the patient discharge level with three potential values: ' 1 ' if the AE (specific to the PSI) likely occurred, ' $O$ ' if the AE did not occur but the patient was considered at risk of such an event occurring, or '? (missing) if the patient was not considered at risk.

Each PSI is represented by a $0 / 1$ outcome of interest variable $Y_{i j t}^{k}$, a discharge-level variable where $i$ indexes the discharge, $j$ indexes the hospital, $t$ indexes time (year), and $k$ indexes the PSI. Therefore $Y_{i j t}^{k}=1$ indicates the occurrence of the AE indicated by PSI $k$ for discharge $i$ treated at hospital $j$ in year $t$.

PSI rates are defined as the incidence of AEs per 1000 at-risk discharges. Their calculation requires the denominator which is the number of at-risk discharges, and the numerator which is the total number of discharges (amongst the at risk population) that are flagged with the adverse event.

PSI rates can be derived at whatever level of aggregation is desired. For example for a given hospital $j$, in year $t$, the observed $\mathrm{PSI}_{\mathrm{k}}$ rate per 1000 discharges $\left(O R_{j t}^{k}\right)$ is calculated as:

$$
O R_{j t}^{k}=\frac{\sum_{i} Y_{i j t}^{k}}{n_{j t}^{k}} * 1000
$$

where $n_{j t}^{k}$ is the denominator, the total number of discharges at risk of $\mathrm{AE} k$, treated at hospital $j$ in year $t . \sum_{i} Y_{i j t}^{k}$ is the numerator, the total number of AEs flagged by PSI $k$ treated at hospital $j$ in year $t$.

The rate of adverse events across the analytical sample is referred to as the "reference population rate" $\left(\alpha_{k}\right)$. This is calculated as follows: 


$$
\alpha_{k}=\frac{\sum_{i} \sum_{j} \sum_{t} Y_{i j t}^{k}}{N_{k}} * 1000
$$

where the numerator represents the total number of AEs identified by $\mathrm{PSI}_{k}$, and the denominator, $N_{k}$ represents the total number of discharges at risk of experiencing the $\mathrm{AE}$

\subsubsection{Aggregate PSIs}

The 20 PSIs can be categorised into four clinical groups: general, medical, post-operative, and obstetrics (Hider, Parker et al. 2014). The medical category is the branch of medicine that deals with non-surgical prevention, diagnosis, and treatment of adult diseases. The post-operative category is for those indicators which focus on the period of time following surgery. The obstetrics indicators are those concerned with childbirth. The general category covers all those indicators that do not fit naturally into the first three categories. In this study five composite PSIs are constructed; four "sub-aggregates" based on the above clinical groupings, and an additional fifth, overall "aggregate" PSI, generated using all 20 PSIs (see Table 4 for details). For the aggregate and sub-aggregate PSIs, a patient discharge is considered at risk if it is at risk of at least one of the individual PSIs which make it up. An $A E$ will be flagged if at least one AE is flagged by any of the individual PSIs.

Table 4: Aggregate PSIs

\begin{tabular}{ll}
\hline PSI Name & Description \\
\hline Overall (AlI) & PSI 1-20 \\
General (Gen) & PSI1, PSI5, PSI6, PSI8, PSI15, PSI16 \\
Medical (Med) & PSI2, PSI3, PSI4, PSI7 \\
Post-op (Post) & PSI9, PSI10, PSI11, PSI12, PSI13, PSI14 \\
Obstetrics (Obst) & PSI17, PSI18, PSI19, PSI20 \\
\hline
\end{tabular}

\subsection{Background to Risk Adjustment}

Risk adjustment is a statistical process that attempts to control for differences in patient characteristics (risk-factors) so that cross-sectional and intertemporal comparisons can be made. Risk adjustment is the basis for which this thesis develops measures of patient safety for New Zealand public hospitals that are considered comparable across hospitals and over time. 
lezzoni proposes that any approach to risk adjustment should start with careful consideration of four questions (lezzoni 2003). Firstly, she categorises risk of outcome into three broad groupings: clinical outcomes, resources used, and patient-centred outcomes. Variables employed as risk adjusters will differ depending on the outcome of interest. Secondly, risks must be framed within some sort of time interval. Clearly defined time frames help to ensure meaningful comparisons are possible. Thirdly, the risks for health outcomes vary across different populations. It is therefore important to clearly define populations of interest, in part because those populations help determine which risk factors are important for comparing outcomes. Finally, the purpose of risk adjustment can vary greatly although the basic motivation is to enable valid comparisons.

This research focuses on clinical outcomes, specifically the adverse events defined by the set of 20 AHRQ PSIs with the purpose of enabling meaningful comparison in hospital quality. The time frame for each adverse event is from admission to discharge. The study considers all admissions (from admission to discharge) in New Zealand public hospitals (2001-2009). Therefore, risk factors pertaining specifically to the New Zealand population, such as ethnicity, and deprivation level are relevant.

\subsubsection{Risk Factors}

"Perhaps the most important feature of any risk-adjustment approach involves its set of risk factors - which risk factors are included and how they are represented and handled analytically." (lezzoni 2003, pg. 33). Risk factors (or risk adjusters) are variables associated with the likelihood of different health outcomes. For example, risk of death increases with age, and gender is related to different risks for certain illnesses and conditions (Knaus, Wagner et al. 1991, Anderson and Statistics 2003). The credibility of results derived from risk adjustment is dictated by the scope of the risk factors included in the model (lezzoni 2003).

lezonni (2003) suggests risk factors can be classified into five broad categories: demographic characteristics (age, sex, ethnicity); clinical factors (principal diagnosis, 
severity of diagnosis, comorbidities); socioeconomic factors (education, employment, deprivation level); health related behaviours and activities (tobacco use, alcohol use, and diet); and attitudes and perceptions (religious beliefs, and preferences and expectations for health care services) (lezzoni 2003).

The use of administrative data restricts the range of risk factors that can be included in risk adjustment models (lezzoni 2003). Administrative data do not typically capture specific information regarding health related behaviours or attitudes and perceptions and New Zealand hospital administrative data are no exception. Therefore the risk adjustment models employed in this study are limited to risk factors associated with demographics, clinical, and socioeconomic factors only. The AHRQ PSI risk adjustment models include sex, age, Diagnosis-Related Groups (DRGs), and comorbidities (AHRQ 2011). New Zealand administrative data include these variables and therefore these risk factors can be replicated. In additional New Zealand specific risk factors, ethnicity and deprivation level are also be explored.

\subsection{Risk Adjustment Modelling}

Due to the complex nature of developing risk adjustment models, lezzoni (2003) strongly advises considering existing methods, either directly or by modifying them for a particular dataset/population (lezzoni 2003). The AHRQ have already developed PSI specific risk adjustment models, a process which was highly resource intensive and had input from numerous specialised researchers (AHRQ 2011). Developing new risk adjustment models is not necessary and is beyond the scope of this research. Therefore in line with recommendations and for pragmatic reasons, the risk adjustment methodology employed is underpinned by the AHRQ PSI risk adjustment version 3.0a (AHRQ 2006).

The AHRQ employ an incremental modelling method using logistic regression based risk adjustment and have extensively researched the most appropriate set of risk factors to control for with respect to each PSI. The incremental modelling approach is advantageous because it permits a greater understanding of the impact of risk factors on the predicative ability of the model. It also makes it possible to attempt to disentangle the risk factor 
specific effects of risk adjustment on hospital level rates of adverse events. In this thesis the AHRQ risk adjustment models are modified for New Zealand data. New Zealand specific risk factors, ethnicity and deprivation level are explored and models re-estimated on New Zealand data.

The AHRQ employ three incremental models, however, the addition of New Zealand specific risk factors means a further level is included in this study. The four incremental models are defined as follows:

1) Sex and age (M1)

2) Sex, age, ethnicity, and deprivation (M2)

3) Sex, age, ethnicity, deprivation, and $\mathrm{DRGs}^{19}(\mathrm{M} 3)$

4) Sex, age, ethnicity, deprivation, DRGs, and comorbidities (M4)

All covariates in the models are constructed as dichotomous $0 / 1$ dummy variables. For sex, one indicates male. Age is split into seven age categories; $<30,30-39,40-49,50-59,60-69$, 70-79, and 80+. Age-sex interactions are also modelled. Ethnicity is derived from the prioritised ethnicity variable in NMDS and split into five ethnic groups: NZ European, Maori, Pacific, Asian, and other. Deprivation level is based on the New Zealand Deprivation Index (NZDep) and categorised into five quintiles. The DRGs included in each model vary depending on the PSI and follow the AHRQ methodology. Comorbidities are modelled in the same way as DRGs. The data set used and many of the variables mentioned above are described in more detail in 4.1.

The aggregate and sub-aggregate indicators are modelled using the same incremental procedures, but due to aggregations the PSI specific DRG covariates are replaced by their aggregated counterparts (MDCs). Obstetrics indicators are not adjusted for DRGs or comorbidities. Therefore only two incremental levels are modelled.

\footnotetext{
${ }^{19}$ Different DRG versions are used in New Zealand compared to those used in the AHRQ models. As a result DRGs from the AHRQ models are mapped manually to the DRGs in NMDS. Some DRGs were not able to be mapped and were therefore excluded from the models. The mapping and subsequent exclusions are detailed in Appendix B.
} 
Age, ethnicity, deprivation, and MDCs are risk factors derived from categorical variables that each require one category to be dropped to avoid suffering from perfect multicollinearity. The categories dropped are the 50-59 age group, the New Zealand European ethnic group, the NZDep $3^{\text {rd }}$ quintile, and MDC5.

An additional covariate selection criterion is also applied: covariates are retained in the model only if they meet a minimum requirement of at least 30 observations that indicate the presence of the risk factor. This is particularly relevant for some of the DRGs which are infrequent. For mutually exclusive risk factors such as age, observations are recoded to the nearest match if required.

\subsection{Estimation Methods}

Three alternative estimation methods are employed: Logistic, Ordinary Least Squares (OLS), and Direct Standardisation (DS). The intention is that the former be the primary estimation method with the latter used as a robustness check.

\subsubsection{Logistic Risk Adjusted Rates}

In order to estimate the incremental models, event-level logistic regressions are run separately for each PSI, using all at-risk admissions from the analytical sample ${ }^{20}$ (irrespective of year and hospital). The PSI outcome variable $Y_{i}$ is regressed on the vector of explanatory variables $X_{i}$ (in the equation notation below superscripts indicating the PSI (k) are omitted for simplicity).

Specifically:

$$
Y_{i}=X_{i} \beta+\zeta_{i}
$$

$Y_{i}$ is the PSI outcome for patient $i$.

$X_{i}$ is a vector of patient covariates for patient $i$.

\footnotetext{
${ }^{20}$ The analytical sample is defined in Section 4.2.
} 
$\beta$ is a vector of parameters, giving the effect of each patient risk adjuster on the outcome of interest.

$\zeta_{i}$ is the error term.

The estimated $\hat{\beta}$ coefficients are used to derive predicted probabilities of the outcome of interest $\left(\hat{P}_{i}\right)$ for each at-risk patient.

Specifically:

$$
\hat{P}_{i}=\exp \left(X_{i} \hat{\beta}\right) /\left(1+\exp \left(X_{i} \hat{\beta}\right)\right)
$$

$\hat{P}_{i}$ should be interpreted as the estimated probability of discharge $i$ experiencing the AE indicated by the corresponding PSI.

Using the predicted values estimated in (4) with subscripts $\mathrm{j}$ and $\mathrm{t}$ included to denote hospital and year respectively (but continuing to omit superscript $k$ to denote the PSI), PSI expected rates (ERs) for each hospital in each year are calculated as follows:

$$
E R_{j t}=\frac{\sum_{i} \hat{P}_{i j t}}{n_{j t}}
$$

$E R_{j t}$ is therefore the ratio of total expected AEs to the total number of discharges at risk of experiencing such an event at hospital $\mathrm{j}$ in year $\mathrm{t}$. The ER is calculated based on the actual casemix that presents at hospital $\mathrm{j}$ in year $\mathrm{t}$, and the estimated probability of each discharge experiencing an $\mathrm{AE}$ as determined by the $\beta$ coefficients estimated from the logistic regression on the analytical sample from (3). Intuitively $E R_{j t}$ can be seen as the PSI rate which would be expected at hospital $j$ in year $t$ if its performance was the same as the analytical sample.

Risk adjusted rates are calculated by taking the ratio of ORs to ERs and multiplying this by the reference population rate. The multiplication simply recalibrates the OR/ER ratio to values that are consistent with rates of adverse events for any given PSI. A hospital that has performed better than expected will have an OR/ER ratio of less than one and therefore its 
recalibrated risk adjusted rate will be better than average (the reference population rate). On the other hand providers that perform worse than expected will have an OR/ER ratio of greater than one and therefore a risk adjusted rate greater than the reference population rate. The PSI Risk Adjusted Rate at hospital $\mathrm{j}$ in year $t\left(R A R_{j t}\right)$ is therefore:

$$
R A R_{j t}=\left(O R_{j t} / E R_{j t}\right) * \alpha
$$

where $\alpha$ is the reference population PSI rate calculated in (2). The risk adjusted rate can be interpreted as the PSI rate a hospital would have if it had the casemix of the reference population.

\subsubsection{Ordinary Least Squares (OLS) Risk Adjusted Rates}

OLS risk adjusted rates are constructed by running event level regressions by $\mathrm{PSI}$, of the 0/1 PSI outcome of interest, on a set of risk factors and a set of hospital/year specific dummy variables. The estimated coefficients of the dummy variables $\left(\alpha_{j t}\right)$ are the OLS risk adjusted rates:

$$
Y_{i j t}=\alpha_{j t} F_{i j t}+\beta X_{i j t}+\varepsilon_{i j t}
$$

$F_{i j t}$ are hospital and year specific dummy variables and $X_{i j t}$ are the set of risk factors.

\subsubsection{Direct Standardisation (DS) Risk Adjusted Rates}

In DS hospital specific observed rates (ORs) are calculated for each of $c$ separate risk stratum $\left(O R_{c j t}\right)$ and applied to the standard population casemix to produce risk adjusted rates. For example, in the simple case when risk adjustment is performed on gender only, the two risk strata would be male and female discharges. The corresponding observed rates are each multiplied by the equivalent proportion of males or females in the reference population. Formally, the DS risk adjusted rate for any given hospital is calculated as:

$$
R A R_{j t}^{D S}=\sum_{c} O R_{c j t} * A S_{c}
$$

where $A S_{c}$ is the proportion of at-risk discharges in stratum c to the total at-risk reference population. 


\subsection{Comparing Alternative Risk Adjustment Methods}

Hider et al. show a number of the PSIs identify relatively rare events when applied to New Zealand data, several below 1 per 10,000 at-risk admissions. Descriptive analysis in Section 4 corroborates these findings. The motivation for the comparison of risk adjusted rates is to attempt to identify any underlying issues that might exist, and assess the accuracy of logistic regression based risk adjustment under these circumstances. To do so logistic regression based risk adjustment is compared to that derived via DS and OLS. Given the limitations of DS with respect to the number of risk factors that can be adjusted for at any one time, $\mathrm{M} 1$ risk adjusted rates are compared ${ }^{21}$.

For each indicator the Pearson correlation coefficients between annual hospital risk adjusted rates derived from each of the three methods are calculated. Spearman Rank correlation coefficients are also calculated. Risk adjusted rates are ordered and ranked from lowest to highest (identical rates are given the same ranking).

Spearman's rank correlation coefficient $(\rho)$ is a nonparametric measure of rank correlation. It assesses how well the relationship between two variables can be described. The Spearman correlation between two variables is equal to the Pearson correlation between the rank values of those two variables.

Intuitively, the Spearman correlation between two variables will be high when observations have a similar rank between the two variables, and low when observations have a dissimilar rank. Identical values are each assigned fractional ranks equal to the average of their positions in the ascending order of the values.

\footnotetext{
${ }^{21}$ An extended analysis for each of the incremental levels of risk adjustment (M1-M4), comparing logistic and OLS risk adjusted rates has also been conducted. The results of this analysis are in line with the analysis presented in the body of the text.
} 


\subsection{Empirical Evaluation of Risk Adjustment Models}

Once a risk adjustment model has been developed the natural question becomes, "How well does it perform?" This section discusses the statistical performance measures used to evaluate the risk adjustment models.

Discrimination measures: sensitivity, specificity, and the c-statistic are calculated for each incremental risk adjustment model. The predicted values estimated from logistic regressions are dichotomised based on a cut-off value (set to the reference population rate of AEs for each indicator). Predicted values greater than or equal to the cut-off indicate a positive outcome, and those less than the cut-off indicate a negative outcome. For this analysis, the cut-off value is set equal to the reference population rate for each indicator. Four pairwise combinations of predicted and observed outcomes are then defined:

A. True Positives - positive predicted outcomes which were also positive observed outcomes

B. False Positives - positive predicted outcomes which were actually negative observed outcomes

C. True Negatives - negative predicted outcomes which were also negative observed outcomes

D. False Negatives - negative predicted outcomes which were actually positive observed outcomes

Sensitivity is calculated as $A /(A+D)$, the fraction of positive outcomes correctly predicted. Specificity equals $C /(B+C)$, the fraction of negative outcomes correctly predicted.

The c-statistic can be defined in several ways. Typically it is described as equal to the area under the ROC curve which is the curve of sensitivity versus (1-specificity) across all prediction cut-off values. The c-statistic can also be derived by taking all possible pairs of patients, one of whom experiences the $A E$ and the other who does not, then finding the proportion of these pairs in which the predictive probability of the patient who experiences the $A E$ is greater than the one who does not. It can be interpreted as the probability that, given two patients, one with the outcome of interest and one without, the patient with the 
event will have a higher predicted value of the outcome. The maximum value of one indicates perfect discrimination while a value of 0.5 indicates random discrimination.

Hosmer and Lemeshow categorise the discriminatory power of models using the c-statistic results (Hosmer and Lemeshow 2000). They suggest models have poor discrimination if the c-statistic is less than 0.7 , acceptable between 0.7 and 0.79 , excellent between 0.8 to 0.89 , and outstanding if the c-statistic is 0.9 or more. This categorisation is used in this study.

Likelihood ratio tests are also employed to compare the fit of the incremental models and test whether to reject the null (nested) model in favour of the alternative model. The test statistic $(D)$ is calculated as follows:

$$
D=-2 \ln \left(\frac{\text { likelihood for null model }}{\text { likelihood for alternative model }}\right)
$$

The null hypothesis $\mathrm{H}_{0}$ (that the null model is true) is rejected in favour of the alternative if $D$ is greater that a critical value chosen from the chi-squared distribution with $\mathrm{j}$ degrees of freedom, where $\mathrm{j}$ is the number of restrictions under $\mathrm{H}_{0}$.

\subsection{Analysis of Hospital Level Risk Adjusted Rates}

Once risk adjustment methods and models have been decided the subsequent hospital level risk adjusted rates are analysed. Firstly, hospital ORs and M1-M4 risk adjusted rates are calculated annually. The resulting distributions of rates (mean, standard deviation, and skewness) are compared across the incremental levels of risk adjustment. The purpose is to attempt to disentangle the effects of each incremental level of risk adjustment on hospital level rates.

Secondly, for each indicator observed and M4 risk adjusted hospital rates are calculated over the full nine years of the study period, ordered and ranked, and five empirical measures generated. These are the Spearman rank correlation coefficient; the average absolute value of the change in hospital ranking; the percentage of hospitals that remain in the top (or bottom) $20 \%$ of the distribution after adjustment; and the percentage of hospitals that change more than two deciles in the distribution after risk adjustment. The 
purpose of this is to understand the effect of the preferred risk adjustment model (M4) on the rankings of hospitals, before and after risk adjustment. 


\section{Section 4 Data}

This study is built around the AHRQ PSIs applied to New Zealand hospital administrative data, the National Minimum Dataset (NMDS). Section 4.1 summarises NMDS, explaining how the data is structured and describing the key variables used in the study. Section $\mathbf{4 . 2}$ describes the data preparation for analysis. Section 4.3 presents descriptive statistics for the ARHQ PSIs applied to NMDS. Descriptive analysis identifying the cost of adverse events is presented in Section 4.4. In Section 4.5 two standard metrics of hospital patient safety and quality employed by the $\mathrm{MoH}$ in New Zealand are presented.

\subsection{National Minimum Data Set (NMDS)}

The data for this study is nine years of the NMDS from 2001 to 2009. NMDS is an administrative dataset and a national collection of New Zealand hospital admissions, including day patients (stays of either three hours or more but not overnight) and inpatients (stays of at least one night). NMDS covers all admissions to publicly funded hospitals and publicly funded events at private hospitals in New Zealand. The terms "observation" and "event" will be used interchangeably throughout the study.

Technically an observation in NMDS corresponds to a single hospital discharge. Therefore, while an observation in NMDS implies a hospital admission has occurred, the event is not recorded until the patient has been discharged. For this reason, "hospital discharge" and "hospital admission" will be used interchangeably when referring to an observation in NMDS. Each observation is uniquely identified by an event ID variable. Each patient can be uniquely identified by his or her encrypted National Health Index (NHI) number. An individual admitted into hospital multiple times will be recorded under the same $\mathrm{NHI}$ number, but the event ID for the separate admissions will differ. This raw dataset contains roughly 7.5 million discharge level observations.

Each observation in NMDS contains a variety of detailed administrative, demographic, clinical, and financial information. Key variables within NMDS for the purpose of this study 
include: the date of admission, the date of discharge, discharge end type, length of stay (LOS), facility (hospital) code, diagnosis codes, Major Diagnostic Category (MDC), Diagnostic Related Group (DRG), age, gender, ethnicity, deprivation level, and domicile. Each of these fields is included in each patient level observation. Several of the variables listed above are further described below.

The majority of the clinical information contained in NMDS comes from diagnosis codes, coded using ICD-10-AM (World Health Organization 2010, National Casemix and Classification Centre 2011). ICD-10-AM is the Australian modification ${ }^{22}$ of the tenth version of the International Statistical Classification of Diseases and Health Related Problems and has been classified and maintained by the WHO since 1948. From 1999 New Zealand began using the ICD-10-AM system. This system governs clinical coding practice and underpins consistency and accuracy of clinical coded information submitted within NMDS (New Zealand Health Information Service 2011). In addition to diagnosis codes, the ICD coding is specifically important in the context of this study as it underpins other key variables used, including DRGs, MDCs, the PSIs and the Elixhauser Comorbidities.

NMDS contains up to 99 diagnosis codes. These can be categorised into the principal diagnosis (the first diagnosis code) and secondary diagnoses (the remaining codes). The principal diagnosis is considered the primary condition for which health care is required. Secondary diagnoses are conditions or complaints that coexist with the principal diagnosis or arise during care. The inclusion of a condition in the observation as a secondary diagnosis code is based on the premise that it impacted materially upon the person's care either through treatments, diagnostic procedures, or increased clinical care and/or monitoring.

The purpose of DRGs is to categorise hospital events into clinically relevant groups which have similar hospital resource use. The DRG system was developed at Yale University with the intention of identifying and categorising the "products" hospitals provide. In a sense DRGs were developed as an accounting measure so that providers and supporting agencies

\footnotetext{
${ }^{22}$ The Australian National Casemix and Classification Centre (NCCC) modified the ICD-10 system specifically for the needs of the Australian health system where various editions have been used since 1998 .
} 
could get a broad sense of what hospitals "produce". In certain cases, the DRG system established a basis for reimbursing hospitals for the services they provide as opposed to previous reimbursement systems purely based on cost. The DRG system has since been revised and adopted by New Zealand and by health care organisations worldwide. For the purposes of this study the DRG system is used to categorise observations into groups of clinical similarity.

Various DRG versions have been used in New Zealand as updates and revisions have been implemented over time developed in Australia by the National Casemix and Classification Centre (NCCC) ${ }^{23}$. Prior to July $1^{\text {st }} 2001$ Australian National Diagnosis Related Groups (ANDRG) 3.1 was used. From July $1^{\text {st }} 2001$ to June $30^{\text {th }} 2002$ AR-DRG 4.1 was in use followed by AR-DRG 4.2 from July $1^{\text {st }} 2002$ to June $30^{\text {th }} 2004$ and AR-DRG 5.0 was used post July $1^{\text {st }}$ 2004. Due to the multiple versions of the DRG system, each observation in NMDS is assigned two separate DRG fields by the Information Directorate of the Ministry of Health (previously the New Zealand Health Information Services). The first is based on the current DRG version at the time of patient hospitalisation, therefore DRG versions may differ across observations. The second DRG field utilises AN-DRG 3.1 and provides a consistent version of the DRG system across all observations. The DRGs are assigned using grouper software which uses a complex web of decision rules to categorise cases into DRGs based on ICD-10AM codes, age, sex, discharge status, and the presence of complications or comorbidities (National Casemix and Classification Centre 2011). This study utilises AN-DRG 3.1 because it is consistent across all observations. The AN-DRG system is hierarchical in the sense that 667 separate DRGs can be grouped into 23 Major Diagnostic Categories (MDCs).

For this study deprivation level is indicated using the New Zealand Deprivation Index (2206), henceforth referred to as NZDep. This is a ten-point index representing deciles of socio economic deprivation, where one represents the least deprived areas, and ten the most deprived. NZDep combines nine variables from New Zealand census results reflecting eight dimensions of deprivation and allocates deprivation levels at the meshblock ${ }^{24}$ level

\footnotetext{
${ }^{23}$ Technical information can be found at http://www.nzhis.govt.nz/moh.nsf/pagesns/318.

${ }^{24} \mathrm{~A}$ meshblock is the smallest geographic unit for which Statistics New Zealand collects data.
} 
(Salmond C, Crampton P et al. 2007). Therefore for each observation in NMDS, a patient's NZDep value is determined by place of residence.

\subsection{Data Preparation}

Several modifications to NMDS have been made since the data were obtained from the $\mathrm{MoH}$. Compass Research Centre of the University of Auckland has generated several additional variables which are now included in the data for this study. The relevant variables created by Compass Research Centre include 30 day mortality, Elixhauser Comorbidities, unplanned (acute) readmission within 30 days, the AHRQ PSIs, and a set of filter variables. The following sections briefly describe these variables, with the exception of the PSIs which are discussed later.

Compass has linked NMDS by encrypted NHI number to mortality records for 2001 to 2007. Only the first seven years of NMDS has been linked, due to the unavailability of mortality data post 2007 at the time the research was conducted. A dummy variable for each observation from 2001 to 2007 reflecting whether or not a patient subsequently died within 30 days of discharge has been generated based on this linked information.

A comorbidity is typically defined as the presence of a disease or condition in addition to a primary disease. A comprehensive set of comorbidity measures was developed for administrative datasets such as NMDS originally using the ICD-9 coding system (Elixhauser, Steiner et al. 1998). These are commonly known as the Elixhauser Comorbidities and comprise a set of 30 independent comorbidities. The algorithms for the Elixhauser Comorbidities were revised for use with ICD-10 coding system in 2005 (Quan, Sundararajan et al. 2005) and have been extensively used in outcome based risk adjustment (Rivard P, Christiansen $C$ et al. 2006). These algorithms have been applied to each observation within NMDS to derive the set of 30 Elixhauser comorbidities (see Table 5 for the complete list). 
Table 5: Elixhauser Comorbidities

\begin{tabular}{llll}
\hline Code & Comorbidity & Code & Comorbidity \\
\hline $\mathbf{1}$ & Congestive heart failure & $\mathbf{1 6}$ & AlDS \\
$\mathbf{2}$ & Cardiac arrhythmias & $\mathbf{1 7}$ & Lymphoma \\
$\mathbf{3}$ & Valvular disease & $\mathbf{1 8}$ & Metastatic cancer \\
$\mathbf{4}$ & Pulmonary circulation disorders & $\mathbf{1 9}$ & Solid tumour without metastasis variation \\
$\mathbf{5}$ & Peripheral vascular disorders & $\mathbf{2 0}$ & Rheumatoid arthritis / collagen vascular \\
& & & diseases \\
$\mathbf{6}$ & Hypertension combined & $\mathbf{2 1}$ & Coagulopathy \\
$\mathbf{7}$ & Paralysis & $\mathbf{2 2}$ & Obesity \\
$\mathbf{8}$ & Other neurological disorders & $\mathbf{2 3}$ & Weight loss \\
$\mathbf{9}$ & Chronic pulmonary disease & $\mathbf{2 4}$ & Fluid and electrolyte disorders \\
$\mathbf{1 0}$ & Diabetes uncomplicated variation & $\mathbf{2 5}$ & Blood loss anaemia \\
$\mathbf{1 1}$ & Diabetes complicated & $\mathbf{2 6}$ & Deficiency anaemias \\
$\mathbf{1 2}$ & Hypothyroidism & $\mathbf{2 7}$ & Alcohol abuse \\
$\mathbf{1 3}$ & Renal failure & $\mathbf{2 8}$ & Drug abuse \\
$\mathbf{1 4}$ & Liver disease & $\mathbf{2 9}$ & Psychoses \\
$\mathbf{1 5}$ & Peptic ulcer disease excluding bleeding & $\mathbf{3 0}$ & Depression \\
\hline
\end{tabular}

An unplanned (or acute) readmission within 30 days of discharge is defined in this study as a patient readmission, via the emergency department, within 30 days of a previous discharge. In NMDS this has been constructed as a 0/1 dummy variable where for a given discharge ' 1 ' indicates a patient experienced an unplanned readmission, and ' 0 ' means they did not. This variable therefore is added ex-post discharge.

The MoH have defined 20 separate criteria called "filters" by which observations can be dropped from analysis utilising information captured by ICD codes (Ministry of Health 2005). This filtering process is advised by the $\mathrm{MoH}$ if providers are being compared, to ensure there is consistent data across providers and over time (the full list of filters can be found in Appendix E). For the purposes of this study five of the 20 filter steps have been applied. These are filters which remove: Non-treated Patients, Error DRGs, Inconsistent Stays, Short Stay ED, and Overseas Patients.

Non-treated patients is a filter for events where no treatment is provided. This might be because operations are cancelled, or a person is admitted as support for a patient receiving care. Such events are not of interest to this study. Error DRGs refer to events which contain 
invalid or atypical clinical coding. These observations are dropped out of concern that the clinical coding will not permit reliable and consistent risk adjustment to be performed. Inconsistent stays are dropped due to conflicts with the timing of how they are recorded and subsequent concerns that information may not be correct. These occur when two events for the same person are recorded at overlapping times. Short stay ED events are dropped because New Zealand Health Information Service (NZHIS) audits found inconsistencies across providers in how the "three-hour rule" was being applied. Some providers were including waiting time and therefore mistakenly treating the case as a day stay when it should have been considered an ED event and not included in NMDS. Finally an overseas patients filter is applied because their demographic information does not fit within our proposed risk adjustment models, namely they cannot be allocated an NZDep score. The remainder of the filters are not applied as there are no obvious negative implications of including them in the analysis.

Applying these five filter steps results in 585,649 observations being dropped leaving $6,901,783$ observations remaining. This filtered dataset is henceforth referred to as the analytical sample.

\subsection{PSI Descriptive Analysis}

The following section provides descriptive analyses on the PSIs. Firstly, it provides basic summary statistics for the aggregate, sub-aggregate, and individual PSIs. This is followed by a descriptive analysis of the PSIs, empirically motivating the case for risk adjustment and examining important risk factors based on New Zealand data: ethnicity and deprivation level. For pragmatic reasons, discussions relating to the PSIs in this and subsequent sections are largely restricted to the aggregate and sub-aggregate PSIs, with reference made to the individual PSI where appropriate. The section includes descriptive analysis summarising the cost of adverse events both in terms of LOS and direct cost of care. Alternative measures of hospital quality are also presented for the study period. 
Table 6 lists each of the 20 PSIs, their respective numerators, denominators, and rates per 1000 at-risk admissions, as well as corresponding statistics for the aggregate and subaggregate PSIs. These statistics are displayed for the analytical sample as well as by gender.

In general, denominators (henceforth referred to as at-risk populations) are large, while numerators (AE numbers) are small; hence the PSIs identify AEs which are infrequent. In the analytical sample the overall PSI aggregate indicator has an at-risk population of over 5.7 million discharges and flags more than $94,000 \mathrm{AEs}$, at a rate of 16.4 per 1000 at-risk admissions.

Table 6: Discharge Level PSIs, Analytical Sample (NMDS 2001-2009)

\begin{tabular}{lrrr}
\hline PSI & Denom. & Num. & Rate \\
\hline Aggregate PSI: All & $5,741,138$ & 94,398 & 16.44 \\
Sub-aggregate PSI: General & $4,768,505$ & 12,528 & 2.63 \\
Sub-aggregate PSI: Medical & $3,201,407$ & 37,821 & 11.81 \\
Sub-aggregate PSI: Postoperative & $1,424,249$ & 31,676 & 22.24 \\
Sub-aggregate PSI: Obstetric & 980,484 & 15,384 & 15.69 \\
\hline PSI1: Complications of anaesthesia & $1,654,181$ & 24 & 0.01 \\
PSI2: Death in low mortality DRGs & $1,777,055$ & 2,150 & 1.21 \\
PSI3: Decubitus ulcer & $1,002,981$ & 17,538 & 17.49 \\
PSI4: Failure to rescue & 98,281 & 10,187 & 103.65 \\
PSI5: Foreign body left during procedure & $4,537,348$ & 345 & 0.08 \\
PSI6: latrogenic pneumothorax & $4,374,030$ & 1,608 & 0.37 \\
PSI7: Selected infection due to medical care & $1,772,919$ & 8,764 & 4.94 \\
PSI8: Postoperative hip fracture & $1,032,261$ & 474 & 0.46 \\
PSI9: Postoperative haemorrhage or haematoma & $1,418,513$ & 26,635 & 18.78 \\
PSI10: Postoperative physiologic and metabolic & 126,531 & 228 & 1.80 \\
derangement & & & \\
PSI11: Postoperative respiratory failure & 92,291 & 131 & 1.42 \\
PSI12: Postoperative pulmonary embolism or DVT & $1,422,601$ & 4,538 & 3.19 \\
PSI13: Postoperative sepsis & 21,329 & 273 & 12.80 \\
PSI14: Postoperative wound dehiscence & 123,952 & 582 & 4.70 \\
PSI15: Accidental puncture of laceration & $4,536,793$ & 10,114 & 2.23 \\
PSI16: Transfusion reaction & 292,500 & 25 & 0.09 \\
PSI17: Birth trauma, injury to neonate & 491,994 & 7,373 & 14.99 \\
PSI18: Obstetrics trauma, vaginal delivery with instrument & 46,002 & 2,884 & 62.69 \\
PSI19: Obstetrics trauma, vaginal delivery without & 323,261 & 4,643 & 14.36 \\
instrument & & & \\
PSI20: Obstetrics trauma, caesarean delivery & 119,227 & 484 & 4.06 \\
\hline & & & \\
\hline & & & \\
& & &
\end{tabular}


Denominators, numerators, and ORs vary across the indicators. At the sub-aggregate indicator level, at-risk populations range from 1.0 million (obstetrics), to 4.8 million (general). While the general indicator has the largest at-risk population, it flags the least AEs of the sub-aggregates with less than 13,000 . In contrast the medical indicator flags nearly 38,000 AEs. The resulting range of rates of AEs is large, from 2.6 per 1000 at-risk admissions (general) to 22.2 per 1000 (post-operative).

In terms of the individual indicators, half have at-risk populations of 1 million admissions or more; the largest is PSI5 (foreign body left during procedure) at 4.5 million. In contrast, several indicators have relatively small at-risk populations, four having less than 100,000 ; PSI13 (post-operative sepsis) has an at-risk population of little more than 21,000. Eight of the PSIs have numerators of less than 500 while four, PSI3 (decubitus ulcer), PSI4 (failure to rescue), PSI9 (post-operative haemorrhage or haematoma) and PSI15 (accidental puncture or laceration) reflect greater than 10,000 potential AEs. PSI1 (complications of anaesthesia) has the lowest rate of AEs at .01 per 1000 admissions. Four other PSIs have rates less than one per 1000. Seven PSIs indicate rates of greater than 10 per 1000, the most frequent being PSI4 at 104 per 1000.

The descriptive results show that the indicators are far from uniform in measurement of their respective adverse events. In particular several indicators flag adverse events at a highly infrequent rate. This has implications for their use as patient safety and quality measures and potentially for risk adjustment also. For example indicators such as PSI1 and PSI16 each flag 25 or fewer adverse events over the study period. Therefore their use should be primarily restricted to monitoring adverse events as a means to flag complications to be investigated further and inform quality improvements. Furthermore, a number of indicators are unlikely to independently differentiate the quality of hospitals at the annual level. The infrequency of the adverse events they flag suggests annual hospital PSI rates will often be zero. Therefore as a tool to differentiate hospital quality their value is restricted. For these reasons the aggregation of indicators (as outlined in 3.1) becomes appealing as a means of utilising the information captured by the individual PSIs. 
The descriptive results also have potential implications for risk adjustment, namely whether logistic regression with rare events can be legitimately applied. However, as discussed in Section 2 , this issue is not solely related to the rarity of the binary dependent variable but is dependent on sample sizes as well. With this in mind the sample sizes (denominators) of each PSI are likely large enough to counter the potential issue relating to the rarity of the adverse events. This is the motivation for the comparison of logistic regression based risk adjusted rates to alternative methods outlined in 3.5.

\subsection{Cost of Adverse Events}

While reducing preventable harm is a major factor in the pursuit of improved patient safety, another significant motivation is that medical error incurs high economic costs. This section investigates if, and by how much, LOS and cost of care differ for admissions flagged with an adverse event compared to those that are not.

Table 7 presents descriptive statistics reflecting the increased LOS and cost of care incurred when adverse events are flagged for the analytical sample. Increased LOS is the difference between ALOS for at-risk events (without a flagged $A E$ ) and those events flagged with an AE. Correspondingly, the cost of AEs is calculated as the difference between mean cost of care for at-risk events to those events flagged with an AE. Weighted Inlier Equivalent Separations ${ }^{25}$ (WIES) methodology is used to estimate cost of care.

For the aggregate indicator ALOS increases by almost eight days reflecting an almost threefold increase when an AE is flagged. Likewise, cost of care increases by just over $\$ 12,000$ on average, again reflecting a more than threefold rise. ALOS and cost of care increases for each of the sub-aggregate indicators and for the individual PSIs although variation in the respective magnitudes is significant. For the sub-aggregates, excluding obstetrics, ALOS increases between 6 to 10 days and corresponding cost of care rises by

\footnotetext{
${ }^{25}$ WIES is a cost weight which represents a relative measure of resource use for each episode of care in a DRG. WIES allocated to an NMDS event depends upon the episode's DRG, the amount of time spent in hospital, time on mechanical ventilation machines, and the episode's eligibility for WIES co-payments. The $2011 / 12$ national casemix price of $\$ 4567.59$ is multiplied by the event level WIES value to derive a proxy for cost of care for each event.
} 
between $\$ 12,000$ and $\$ 16,000$. On the other hand, ALOS for the obstetrics sub-aggregate indicator increases by less than one day and the corresponding increase in cost of care is under $\$ 1000$.

Table 7: Increase in ALOS and Cost of Care due to Adverse Events.

\begin{tabular}{|c|c|c|c|c|}
\hline Indicator & $\begin{array}{r}\text { ALOS } \\
\text { (no AE) }\end{array}$ & $\begin{array}{r}\text { Increased } \\
\text { ALOS } \\
\text { (with AE) }\end{array}$ & $\begin{array}{r}\text { Cost of } \\
\text { care (No } \\
\text { AE) }\end{array}$ & $\begin{array}{r}\text { Increased } \\
\text { Cost } \\
\text { (with AE) }\end{array}$ \\
\hline PSI All & 3.45 & 7.86 & $\$ 4,685$ & $\$ 12,104$ \\
\hline PSI General & 3.80 & 6.10 & $\$ 5,397$ & $\$ 12,857$ \\
\hline PSI Medical & 5.15 & 9.76 & $\$ 6,450$ & $\$ 11,909$ \\
\hline PSI Surgical & 3.40 & 8.89 & $\$ 7,696$ & $\$ 15,591$ \\
\hline PSI Obstetrics & 2.72 & 0.76 & $\$ 2,714$ & $\$ 793$ \\
\hline PSI1: Complications of Anaesthesia & 3.47 & 4.87 & $\$ 7,476$ & $\$ 6,603$ \\
\hline PSI2: Death in Low Mortality DRGs & 2.56 & 3.71 & $\$ 3,916$ & $\$ 8,270$ \\
\hline PSI3: Decubitus Ulcers & 11.60 & 8.44 & $\$ 12,398$ & $\$ 7,651$ \\
\hline PSI4: Failure to Rescue & 9.93 & 0.24 & $\$ 12,996$ & $\$ 6,517$ \\
\hline PSI5: Foreign Body Left During Procedure & 3.88 & 4.02 & $\$ 5,504$ & $\$ 10,194$ \\
\hline PSI6: latrogenic Pneumothorax & 3.73 & 6.97 & $\$ 5,158$ & $\$ 13,009$ \\
\hline PSI7: Selected Infections due to Medical Care & 7.36 & 5.19 & $\$ 8,520$ & $\$ 7,902$ \\
\hline PSI8: Postoperative Hip Fracture & 2.85 & 17.19 & $\$ 6,902$ & $\$ 12,913$ \\
\hline PSI9: Postoperative Haemorrhage or Haematoma & 3.45 & 7.83 & $\$ 7,777$ & $\$ 14,485$ \\
\hline $\begin{array}{l}\text { PSI10: Postoperative Physiologic and Metabolic } \\
\text { Derangements }\end{array}$ & 4.33 & 18.87 & $\$ 10,173$ & $\$ 50,166$ \\
\hline PSI11: Postoperative Respiratory Failure & 4.23 & 23.59 & $\$ 8,184$ & $\$ 77,634$ \\
\hline PSI12: Postoperative Pulmonary Embolism or DVT & 3.55 & 13.66 & $\$ 7,981$ & $\$ 18,633$ \\
\hline PSI13: Postoperative Sepsis & 12.12 & 14.48 & $\$ 23,447$ & $\$ 31,590$ \\
\hline PSI14: Postoperative Wound Dehiscence & 8.47 & 13.60 & $\$ 14,335$ & $\$ 18,437$ \\
\hline PSI15: Accidental Puncture Or Laceration & 3.86 & 5.50 & $\$ 5,475$ & $\$ 12,798$ \\
\hline PSI16: Transfusion Reaction & 7.10 & 16.46 & $\$ 11,505$ & $\$ 30,210$ \\
\hline PSI17: Birth Trauma - Injury to Neonate & 2.90 & 1.29 & $\$ 2,407$ & $\$ 1,027$ \\
\hline $\begin{array}{l}\text { PSI18: Obstetric Trauma - Vaginal Delivery With } \\
\text { Instrument }\end{array}$ & 2.59 & 0.52 & $\$ 2,298$ & $\$ 1,197$ \\
\hline $\begin{array}{l}\text { PSI19: Obstetric Trauma - Vaginal Delivery } \\
\text { Without Instrument }\end{array}$ & 1.79 & 0.66 & $\$ 2,008$ & $\$ 1,260$ \\
\hline PSI20: Obstetric Trauma - Caesarean Delivery & 4.58 & 0.39 & $\$ 6,012$ & $\$ 967$ \\
\hline
\end{tabular}

Table 7 shows more variation exists with respect to the individual PSIs. For the majority of the PSIs ALOS and cost of care either doubles, triples or quadruples with the occurrence of an AE. Notable exceptions include PSI8, PSI10, PSI11, \& PSI12, all post-operative (surgical) indicators. The ALOS for these indicators ranges from 17 to 24 days reflecting 400 to 600 
percent increases. Likewise, cost of care also tends to increase by larger magnitudes; $\$ 50,000$ and $\$ 70,000$ for PSIs 10 and 11 respectively. In contrast relatively small increases in ALOS and cost are evident for the obstetrics PSIs. Typically ALOS increases by half a day for mothers after an obstetrics trauma and related increases in costs of care total around $\$ 1,000$. Injury to neonates increases ALOS by roughly one day and associated increases in costs are also about $\$ 1,000$.

One way to calculate an overall cost to New Zealand public hospitals, albeit a crude one, is to multiply both the increased LOS and cost of care by the number of adverse events flagged for each individual PSI and then sum over the 20 PSIs. This suggests a potentially avoidable increase in bed days from 2001 to 2009 of 584,921 days and related increase in cost of care of 977 million dollars across the provider arm of the New Zealand public health sector.

\subsection{Unplanned Readmissions and 30-day Mortality}

Administrative data also permits alternative means of measuring hospital quality. The most common are unplanned readmission rates and 30 day mortality rates ${ }^{26}$. Unplanned readmissions within 30 days is often used as a metric for quality of care in terms of care effectiveness and safety of care. This is based on the premise that safe and effective hospital care should be associated with lesser chances of patients being readmitted. The 30-day mortality rate is also a commonly used metric of hospital quality potentially indicating the level of safety and effectiveness of care provided ${ }^{27}$. Table 8 displays these metrics for the analytic sample annually from 2001 to 2009 including ALOS for reference.

Unplanned readmissions have increased by 6.3 percent from 10.7 percent of all admissions in 2001 to 11.4 percent in 2009. On the other hand 30-day mortality rates have decreased by 10.2 percent from a rate of 2.3 percent in 2001 to 2.1 in 2009 . At the same time ALOS has declined over the study period by 9.1 percent from 3.4 to 3.1 days.

\footnotetext{
${ }^{26}$ Mortality rates have been constructed until 2007 only, due to the lag in mortality data availability which would permit linkage of mortality records at the time of construction.

${ }^{27}$ Mortality rates presented are not risk adjusted. As a result caution must be taken when drawing any conclusions from the results.
} 
Table 8: ALOS, Unplanned Readmissions, and 30-day Mortality

\begin{tabular}{lrrr}
\hline Year & ALOS & $\begin{array}{r}\text { Unplanned } \\
\text { Readmissions }\end{array}$ & $\begin{array}{r}\text { 30-day } \\
\text { Mortality }\end{array}$ \\
\hline 2001 & 3.44 & 10.74 & 2.33 \\
2002 & 3.43 & 10.80 & 2.31 \\
2003 & 3.39 & 10.87 & 2.31 \\
2004 & 3.32 & 10.85 & 2.31 \\
2005 & 3.27 & 10.83 & 2.16 \\
2006 & 3.19 & 11.05 & 2.26 \\
2007 & 3.22 & 11.11 & 2.09 \\
2008 & 3.18 & 11.23 &. \\
2009 & 3.13 & 11.41 &. \\
\% change 01/09 & $\mathbf{- 9 . 1 \%}$ & $\mathbf{6 . 3 \%}$ & $\mathbf{- 1 0 . 2 \%}$ \\
\hline
\end{tabular}

In terms of quality of care metrics, the trends of unplanned readmissions and 30-day mortality are inconsistent. The former suggests effectiveness and safety of care may have declined while the later indicates the opposite. Declining ALOS may go some way to explaining the rise in unplanned readmissions; a consequence of discharging patients too early may be that readmissions are more likely. However, the same argument would suggest mortality rates would have worsened over time and that is not the case. One way to reconcile this could be to acknowledge the complexity of health care and as a result not all measures of quality of care will trend in the same direction. 


\section{Section 5 Results}

In this section the results from risk adjustment are presented. Section 5.1 presents the results of descriptive analysis of the PSIs intended to empirically motivate risk adjustment. Section 5.2 presents logistic regression results for incremental models of risk adjustment across each of the indicators. Coefficient estimates and their respective standard errors are interpreted and discussed. Empirical evaluation of the incremental models using c-statistics, and LR tests on nested models are presented in Section 5.3. In Section 5.4 the results of comparing risk adjusted rates derived from logistic regression to OLS and DS based risk adjustment are reported. Finally, the effects of risk adjustment on hospital level rates of adverse events are presented in Section 5.5.

\subsection{Descriptive Analysis of Risk Factors}

The importance of risk adjustment for outcome-based measures of hospital performance stems from the likelihood that certain populations are more at risk than others. In order to investigate the importance of risk adjustment for the AHRQ PSIs applied to NMDS, rates are stratified by potential risk factors and relative rates examined. Chi-square tests of independence are conducted for each PSI/risk factor combination and the Pearson correlation coefficient (and level of significance) between patient level indicators and dichotomous and ordinal risk factors is calculated. While the AHRQ risk-adjusted models are adopted, the potential to modify them for New Zealand data is also assessed. As a result the descriptive analysis doubles as a preliminary exploratory exercise for New Zealand specific risk factors that might be included in the models. As described in 3.3 the risk factors to be explored in this study are sex, age, ethnicity, deprivation level, DRGs, and comorbidities. Due to the high number of individual DRGs included in the AHRQ risk adjustment models (200+), for the initial descriptive analysis, MDCs (23 groups) are examined in order to make the analysis more manageable.

Each set of risk factors is examined and discussed separately with stratified results presented in the appendices. Table 9 reports the $p$-values of Chi-squared tests of association of $0 / 1$ indicators for each PSI with various risk factors. The key findings of the analysis are that gender, 
patient age, DRGs, comorbidities, and to a lesser extent ethnicity and deprivation level appear to be significantly associated with the incidence of AEs and therefore might confound measures of hospital performance.

Table 9: PSI Chi2 Tests of Association (p-values)

\begin{tabular}{lrrrrrr}
\hline PSI & $\mathbf{N}$ & Gender & Age & Ethnicity & NZDep & MDC \\
\hline All & $5,741,138$ & 0.000 & 0.000 & 0.000 & 0.000 & 0.000 \\
Gen & $4,768,505$ & 0.000 & 0.000 & 0.000 & 0.000 & 0.000 \\
Med & $3,201,407$ & 0.000 & 0.000 & 0.000 & 0.000 & 0.000 \\
Post & $1,424,249$ & 0.000 & 0.000 & 0.000 & 0.003 & 0.000 \\
Obst & 980,484 & 0.000 & 0.000 & 0.000 & 0.000 & 0.000 \\
\hline PSI1 & $1,654,181$ & 0.219 & 0.147 & 0.565 & 0.578 & 0.009 \\
PSI2 & $1,777,055$ & 0.006 & 0.000 & 0.000 & 0.063 & 0.000 \\
PSI3 & $1,002,981$ & 0.001 & 0.000 & 0.000 & 0.013 & 0.000 \\
PSI4 & 98,281 & 0.045 & 0.000 & 0.000 & 0.000 & 0.000 \\
PSI5 & $4,537,348$ & 0.989 & 0.000 & 0.596 & 0.159 & 0.000 \\
PSI6 & $4,374,030$ & 0.000 & 0.000 & 0.103 & 0.016 & 0.000 \\
PSI7 & $1,772,919$ & 0.000 & 0.000 & 0.001 & 0.468 & 0.000 \\
PSI8 & $1,032,261$ & 0.001 & 0.000 & 0.000 & 0.006 & 0.000 \\
PSI9 & $1,418,513$ & 0.000 & 0.000 & 0.000 & 0.051 & 0.000 \\
PSI10 & 126,531 & 0.000 & 0.000 & 0.000 & 0.033 & 0.000 \\
PSI11 & 92,291 & 0.032 & 0.000 & 0.000 & 0.615 & 0.000 \\
PSI12 & $1,422,601$ & 0.327 & 0.000 & 0.468 & 0.000 & 0.000 \\
PSI13 & 21,329 & 0.445 & 0.289 & 0.081 & 0.469 & 0.000 \\
PSI14 & 123,952 & 0.000 & 0.000 & 0.608 & 0.290 & 0.000 \\
PSI15 & $4,536,793$ & 0.000 & 0.000 & 0.000 & 0.000 & 0.000 \\
PSI16 & 292,500 & 0.011 & 0.137 & 0.197 & 0.236 & 0.236 \\
PSI17 & 491,994 & 0.000 & N/A & 0.000 & 0.000 & N/A \\
PSI18 & 46,002 & N/A & 0.000 & 0.000 & 0.000 & N/A \\
PSI19 & 323,261 & N/A & 0.000 & 0.000 & 0.000 & N/A \\
PSI20 & 119,227 & N/A & 0.010 & 0.130 & 0.554 & N/A \\
\hline & & & & & &
\end{tabular}

In general male patients are more likely to experience an AE than females. The overall aggregate PSI suggests males experience AEs at a rate of 17.9 per 1000 admissions, compared to 15.4 for females. This differential is particularly evident in the post-operative indicator, where men experience AEs at a rate of 24.7 per 1000 admissions, compared to 19.9 for women, and in the medical PSI which shows corresponding rates of 13.2 and 10.7 respectively. At the individual PSI level, 13 of 17 PSIs display a higher incidence of AEs for males than females (note that PSIs 18-20 are obstetrics related and applicable to females only). Chi-square tests 
reject the null hypothesis that the incidence of $A E s$ and gender are independent (at the five percent level of significance). Chi-square tests reject the null hypothesis for each of the subaggregates and 13 out of 17 individual PSIs.

The association between age and adverse outcomes is well documented and for the ARHQ PSIs applied to NMDS the incidence of AEs increases with age. Rates are stratified into seven age categories where the aggregate indicator shows the rate of AEs roughly doubles from the youngest age category (11.5 per 1000) to the oldest (23.7 per 1000) (see Table 16 in Appendix C). Independence between AEs and age is rejected for the aggregate indicator, each of the subaggregates, and all but three of the individual PSIs.

There are divided opinions about whether to include ethnicity in risk adjustment models. Ethnicity was not included in AHRQ risk adjustment models because the researchers wanted to create models which were not confined to use in the United States only. It can be argued that certain ethnic groups might receive lower quality care than others and as a result including ethnicity as a risk factor could mask important differences in quality. However ethnicity may be associated with poorer outcomes due to biological considerations which are independent of quality of care and for this reason it is explored as a possible risk factor in these models. The indicators tend to exhibit significant variation in ORs across ethnic groups, however trends are not consistent across indicators (see Table 17 in Appendix C). The Chi-square test of independence is rejected for the aggregate and sub-aggregate indicators, as well as 12 of 20 individual PSIs. The aggregate indicator shows Asian patients have the highest incidence of AEs (19.2 per 1000), however this is driven solely by obstetrics events, with rates of events for the other sub-aggregates all at or below the reference population rate. Maori patients are on average subjected to the fewest AEs (12.6 per 1000) and have lower than average rates in each of the four sub-aggregate indicators. Across the range of indicators New Zealand Europeans tend to experience more AEs with higher than average rates at the aggregate level, in three of four sub-aggregates, and 13 of 20 individual PSIs.

Those deemed most deprived may be more likely to experience adverse outcomes as a result of potentially poorer health states and other socio economic related issues. NZDep is therefore 
explored as a possible risk factor. Systematic variation in the distribution of PSI rates across NZDep quintiles is apparent although variation differs across indicators (see Table 18 in Appendix C). At the aggregate level, the Chi-square test of independence between AEs and deprivation level is rejected. The aggregate indicator shows rates of AEs are relatively constant across the first four quintiles of deprivation, but decrease sharply for patients in the upper quintile (most deprived). Chi-square tests are also rejected for each of the four sub-aggregates but for only half of the individual PSIs.

As per the AHRQ PSI risk adjustment models, DRGs are a risk factor representing clinical aspects for each admission. However there are too many to analyse individually. Instead, in order to understand how clinical factors affect the incidence of $A E s$, indicator rates are stratified by MDC (see Table 17 in Appendix C).

MDCs are typically associated with significantly different rates of AEs. For the aggregate indicator, the Chi-square test is rejected. Several MDCs display rates of AEs significantly deviating from the reference population rate: MDC18: Infectious and parasitic diseases (37.7 per 1000) displays the highest rate of AEs, more than twice the reference population average; an additional five MDC categories are at least 50 percent higher than average; MDC19: Mental diseases and disorders (3.1 per 1000) has the most infrequent rate of AEs, less than 25 percent of the overall average; and a further three MDCs are 50 percent below the average. Furthermore, Chi-square tests are rejected for each of the sub-aggregate indicators, as well as all but one of the individual PSIs (PSI16).

The AHRQ also employs the use of comorbidities in its risk adjustment models. PSI rates stratified by the presence or otherwise of 27 different comorbidities demonstrate a strong association between comorbidities and increased rates of AEs (see Table 20 in Appendix C). For the aggregate indicator, the presence of any comorbidity typically results in at least a two-fold increase in the rate of AEs; the presence of pulmonary circulation disorders (comorbidity 4 ) has a rate of AEs more than eight times greater than without. These results are largely reflected in the remainder of both the sub-aggregates and individual PSIs. Comorbidities having the most 
impact on the incidence of AEs include comorbidity 1 (congestive heart failure), comorbidity 14 (liver disease), and comorbidity 23 (weight loss).

Gender, age, DRGs, and comorbidities are all included in the AHRQ risk adjustment models. The empirical evaluation presented, examining the association between each of these risk factors and the incidence of AEs, strongly supports their inclusion in risk adjustment models with New Zealand data. Likewise, the empirical evidence supports the inclusion of additional New Zealand specific risk factors: ethnicity and deprivation level. Therefore, there is a strong case for the inclusion of all of the variables in the risk adjustment of the PSIs.

\subsection{Regression Results}

This subsection begins by providing some background to interpreting logistic regression estimation results in 5.2.1. Specific issues regarding interpretation, stemming from the construction of the risk adjustment models, are addressed. The regression results are then discussed and interpreted in 5.2.2.

\subsubsection{Background to Interpreting Logistic Regression Results}

Logistic regressions are conducted for each of the four incremental models across each of the aggregate, sub-aggregate, and individual PSIs. Estimation results suggest that individual risk factors are generally statistically significant, while the magnitudes of estimated coefficients of risk factors vary considerably. For pragmatic reasons discussion focuses on the aggregate indicator (unless otherwise stated) as it is generally representative of the remainder of the indicators. The regression results for the aggregate indicator across each of the four incremental models are displayed in Table 10. Results for all other indicators can be found in Appendix D.

The estimated coefficients in a logistic regression model are not as easy to interpret as standard OLS regression estimates. Logistic regression coefficient estimates signal the relationship between independent variables and the dependent variable which is on a logit scale. Therefore, coefficient estimates indicate the change in the predicted log odds of the 
outcome of interest from a one unit increase in a covariate, holding all other covariates constant.

Coefficients are in log-odds units and as they are difficult to interpret they are often converted into odds ratios. However, prior to this conversion, the sign of the coefficient estimate has intuitive appeal. A positive coefficient estimate indicates the probability of the outcome of interest occurring will increase from a one unit increase in the covariate, and a negative sign specifies a decrease in probability. For example, for the aggregate PSI, the coefficient estimate for obesity (in M4) is 0.405 . This indicates the probability of an adverse event will increase when the patient is obese compared to when the patient is not, holding all else constant.

Not all coefficient estimates from the risk adjustment models can be interpreted in the way described above. First, covariates derived from categorical variables with more than two levels that are defined as dummy variables, such as ethnicity, must be treated slightly differently. In these cases the interpretation of the coefficient estimate and the subsequent odds ratio must be relative to the reference category, the omitted dummy variable. In the case of ethnicity, the reference category is New Zealand European. Therefore the coefficient estimate for Maori (in M2) which is -0.079 and indicates the probability of an AE for Maori decreases compared to New Zealand Europeans.

Second, complications arise due to interactions included in the model for age and gender. When interactions are included in a model, the effect of a one unit change in a covariate is no longer simply a function of the estimated coefficient, rather it is dependent on the additional covariate included in the interaction. For example, the interpretation of the coefficient estimate for "age $<30$ " is dependent on gender as well. In this case, the estimated coefficient for age $<30$ is -0.346 , which indicates that for females, the predicted probability of an $A E$ decreases for those younger than 30 relative to the reference category. However for males, the interaction coefficient must also be considered. In this case it is the linear combination of the two coefficient estimates that determines the change in predicted probability of an AE for males aged less than 30 relative to males aged 50-59. 


\subsubsection{Regression Results and Interpretation}

Overall, regression results show a systematic trend that as age increases the predicted probability of an AE increases, and this is evident across all of the indicators. This is in line with previous descriptive results from Section 5.1. For the aggregate indicator, estimated M4 coefficients for the age dummies are all statistically significant. Furthermore for those dummies reflecting age groups younger than the reference category (50-59) the coefficient estimates are negative and increase in absolute value as the age category becomes younger. On the other hand, for those age categories reflecting age groups older than the reference category, coefficient estimates are positive and increase in magnitude as age increases. This means that for female patients, the predicted probability of an AE increases with age. The corresponding odds ratio for age $<30$ is 0.59 indicating that relative to females aged $50-59$ the predicted odds of an $A E$ is 41 percent lower. At the other end, for female patients aged 80 and over, the corresponding odds ratio is 1.45 reflecting a 45 percent increase in the predicted odds of an AE. For M4 risk adjustment only two of the six age-sex interactions are statistically significant. This signifies that at five percent level of significance one cannot reject the null hypothesis that the age-gender interaction is equal to zero.

Interpreting the regression results for gender is challenging due to the interactions with age. For the aggregate PSI the coefficient estimate for male is positive and statistically significant but due to the interaction terms with age this indicates that the predicted probability of an $A E$ increases for males aged 50-59 compared to women in the same age bracket. To get a fuller interpretation of the effect of gender one must calculate the linear combination of the coefficient for gender and for each respective age-sex interaction term. For the aggregate PSI all are positive and generally statistically significant signalling that the predicted probability of an $A E$ does increase when gender is male across all of the models age categories. These results are not consistent across the remainder of the models. For the sub-aggregates and the individual PSIs coefficients the effect of gender on the predicted probability is AE specific. Statistical significance exists for some indicators but not others and the signs of the coefficients vary. For some indicators the predicted probability of the respective $A E$ increases when gender 
is male, for others it decreases, and for many the effect of gender differs across the age categories. These results are generally consistent with prior descriptive statistics.

The coefficient estimates for the ethnic group dummies are inconsistent across the indicators in terms of magnitude, sign, and statistical significance. There does not appear to be any obvious pattern although in most models the majority of the coefficients are statistically significant at the five percent level. For the aggregate PSI, the coefficients for each of the ethnic groups, with the exception of the "other" category are all statistically significant, however the signs on the coefficients differ. The coefficient for Maori is negative, indicating that relative to the New Zealand European reference category, the predicted probability of an AE for patients who identify as Maori decreases. In contrast the coefficients for Pacific and Asian are both positive, signifying an increase in the predicted probability of an adverse event. Overall this suggests that while there appears to be no systematic association between ethnicity and the occurrence of adverse events on an indicator by indicator basis ethnicity is an important predictor.

As with ethnicity, coefficient estimates for deprivation level quintiles are not consistent across indicators, however some patterns do emerge. For the aggregate indicator, coefficient estimates for the fourth and upper quintiles are negative, statistically significant, and increase in magnitude (in absolute terms) as deprivation increases. This implies that as deprivation increases, relative to the reference category (the $3^{\text {rd }}$ quintile), the predicted probability of an AE decreases. 
Table 10: Aggregate Indicator Modelling Results

\begin{tabular}{|c|c|c|c|c|c|c|}
\hline Variables & Mean & $\begin{array}{l}\text { Std. } \\
\text { Dev. }\end{array}$ & M1 & M2 & M3 & M4 \\
\hline Overall Aggregate (All) & 0.016 & 0.127 & & & & \\
\hline Male & 0.431 & 0.495 & 0.0301 & 0.0322 & $0.078 * * *$ & $0.080 * * *$ \\
\hline Age $<30$ & 0.229 & 0.420 & $-0.346 * * *$ & $-0.358 * * *$ & $-0.690 * * *$ & $-0.525 * * *$ \\
\hline Age 30-39 & 0.133 & 0.340 & $-0.301 * * *$ & $-0.322 * * *$ & $-0.545^{* * *}$ & $-0.411 * * *$ \\
\hline Age 40-49 & 0.115 & 0.319 & $-0.190 * * *$ & $-0.197^{* * *}$ & $-0.291 * * *$ & $-0.221 * * *$ \\
\hline Age 60-69 & 0.133 & 0.339 & $0.232 * * *$ & $0.235^{* * *}$ & $0.271^{* * *}$ & $0.209 * * *$ \\
\hline Age 70-79 & 0.154 & 0.361 & $0.436 * * *$ & $0.439 * * *$ & $0.478 * * *$ & $0.376 * * *$ \\
\hline Age $80+$ & 0.131 & 0.337 & $0.470 * * *$ & $0.474 * * *$ & $0.487 * * *$ & $0.373 * * *$ \\
\hline Male Age $<30$ & 0.082 & 0.275 & $0.207^{* * *}$ & $0.203 * * *$ & $0.169 * * *$ & $0.170 * * *$ \\
\hline Male Age 30-39 & 0.035 & 0.184 & $-0.230 * * *$ & $-0.214 * * *$ & 0.001 & -0.001 \\
\hline Male Age 40-49 & 0.059 & 0.236 & $-0.070 * *$ & $-0.0651 * *$ & 0.021 & 0.0267 \\
\hline Male Age 60-69 & 0.071 & 0.257 & $0.112^{* * *}$ & $0.111^{* * *}$ & $0.079 * * *$ & $0.076 * * *$ \\
\hline Male Age 70-79 & 0.081 & 0.273 & $0.060 * *$ & $0.0588^{* *}$ & 0.031 & 0.011 \\
\hline Male Age $80+$ & 0.054 & 0.226 & -0.000 & -0.00302 & -0.015 & $-0.050^{*}$ \\
\hline Maori & 0.149 & 0.356 & & $-0.0786 * * *$ & $-0.031 * * *$ & $-0.122 * * *$ \\
\hline Pacific & 0.060 & 0.238 & & $0.143 * * *$ & $0.142 * * *$ & $0.044 * * *$ \\
\hline Asian & 0.044 & 0.206 & & $0.352 * * *$ & $0.340 * * *$ & $0.329 * * *$ \\
\hline Other & 0.115 & 0.319 & & 0.00176 & 0.001 & 0.014 \\
\hline NZDep (lower quintile) & 0.136 & 0.343 & & -0.00831 & $-0.020^{*}$ & -0.009 \\
\hline NZDep (second quintile) & 0.160 & 0.366 & & 0.00153 & -0.006 & 0.004 \\
\hline NZDep (fourth quintile) & 0.241 & 0.428 & & $-0.0212 * *$ & $-0.017^{*}$ & $-0.022 * *$ \\
\hline NZDep (upper quintile) & 0.265 & 0.441 & & $-0.0466 * * *$ & $-0.032 * * *$ & $-0.039 * * *$ \\
\hline MDC1 Nervous system & 0.052 & 0.223 & & & $-0.214 * * *$ & $-0.043 * *$ \\
\hline MDC2 Eye & 0.025 & 0.157 & & & $-1.704 * * *$ & $-1.292 * * *$ \\
\hline MDC3 Ear, nose, mouth and throat & 0.026 & 0.158 & & & $-0.502 * * *$ & $-0.219 * * *$ \\
\hline MDC4 Respiratory system & 0.061 & 0.240 & & & $-0.264 * * *$ & $-0.291 * * *$ \\
\hline MDC6 Digestive system & 0.102 & 0.302 & & & $-0.104 * * *$ & $0.068 * * *$ \\
\hline $\begin{array}{l}\text { MDC7 Hepatobiliary system and } \\
\text { pancreas }\end{array}$ & 0.020 & 0.142 & & & $0.366 * * *$ & $0.415 * * *$ \\
\hline MDC8 Musculoskeletal system & 0.085 & 0.279 & & & $0.0423 * * *$ & $0.289 * * *$ \\
\hline $\begin{array}{l}\text { MDC9 Skin, subcutaneous tissue and } \\
\text { breast }\end{array}$ & 0.052 & 0.221 & & & $-0.629 * * *$ & $-0.349 * * *$ \\
\hline $\begin{array}{l}\text { MDC10 Endocrine, nutritional and } \\
\text { metabolic }\end{array}$ & 0.012 & 0.109 & & & $0.257 * * *$ & $0.146 * * *$ \\
\hline MDC11 Kidney and urinary tract & 0.063 & 0.243 & & & $-0.566 * * *$ & $-0.287 * * *$ \\
\hline MDC12 Male reproductive system & 0.008 & 0.088 & & & $0.253 * * *$ & $0.507 * * *$ \\
\hline MDC13 Female reproductive system & 0.032 & 0.176 & & & $0.460 * * *$ & $0.716 * * *$ \\
\hline MDC14 Pregnancy & 0.124 & 0.330 & & & $0.208 * * *$ & $0.476 * * *$ \\
\hline MDC15 Newborn and other neonates & 0.086 & 0.280 & & & $0.443 * * *$ & $0.700 * * *$ \\
\hline
\end{tabular}

*** $p<0.01,{ }^{* *} p<0.05,{ }^{*} p<0.1$ 
Table 10: Aggregate Indicator Modelling Results (continued)

\begin{tabular}{|c|c|c|c|c|c|c|}
\hline Variables & Mean & $\begin{array}{l}\text { Std. } \\
\text { Dev. }\end{array}$ & M1 & M2 & M3 & M4 \\
\hline $\begin{array}{l}\text { MDC16 Blood and immunological } \\
\text { disorders }\end{array}$ & 0.014 & 0.117 & & & $-1.164 * * *$ & $-0.951 * * *$ \\
\hline MDC17 Neoplastic disorders & 0.023 & 0.150 & & & $-0.881 * * *$ & $-0.717 * * *$ \\
\hline $\begin{array}{l}\text { MDC18 Infectious and parasitic } \\
\text { diseases }\end{array}$ & 0.013 & 0.112 & & & $0.766 * * *$ & $0.837^{* * *}$ \\
\hline MDC19 Mental diseases and disorders & 0.013 & 0.114 & & & $-1.546 * * *$ & $-1.370 * * *$ \\
\hline MDC20 Alcohol/drug & 0.002 & 0.046 & & & $-1.128 * * *$ & $-1.175 * * *$ \\
\hline MDC21 Injuries, poisoning & 0.024 & 0.152 & & & $0.144 * * *$ & $0.349 * * *$ \\
\hline MDC22 Burns & 0.001 & 0.031 & & & $0.430 * * *$ & $0.657^{* * *}$ \\
\hline MDC23 Other Factors & 0.043 & 0.204 & & & $0.209 * * *$ & $0.181 * * *$ \\
\hline Congestive heart failure & 0.029 & 0.168 & & & & $0.644 * * *$ \\
\hline Valvular disease & 0.014 & 0.117 & & & & $0.350 * * *$ \\
\hline Pulmonary circulation disorders & 0.004 & 0.064 & & & & $1.882 * * *$ \\
\hline Peripheral vascular disorders & 0.014 & 0.119 & & & & $0.911 * * *$ \\
\hline Hypertension combined & 0.107 & 0.309 & & & & $0.340 * * *$ \\
\hline Paralysis & 0.021 & 0.143 & & & & $-0.200 * * *$ \\
\hline Other neurological disorders & 0.017 & 0.129 & & & & $0.471 * * *$ \\
\hline Chronic pulmonary disease & 0.024 & 0.153 & & & & $0.274^{* * *}$ \\
\hline Diabetes uncomplicated variation & 0.036 & 0.187 & & & & $-0.083 * * *$ \\
\hline Diabetes complicated & 0.040 & 0.196 & & & & $0.136 * * *$ \\
\hline Hypothyroidism & 0.003 & 0.055 & & & & $0.101 * *$ \\
\hline Renal failure & 0.060 & 0.237 & & & & 0.002 \\
\hline Liver disease & 0.006 & 0.080 & & & & $0.922 * * *$ \\
\hline Peptic ulcer disease excluding bleeding & 0.001 & 0.031 & & & & $0.517 * * *$ \\
\hline AIDS & 0.000 & 0.017 & & & & -0.107 \\
\hline Lymphoma & 0.006 & 0.079 & & & & $0.170 * * *$ \\
\hline Metastatic cancer & 0.023 & 0.151 & & & & $0.879 * * *$ \\
\hline $\begin{array}{l}\text { Solid tumour without metastasis } \\
\text { variation }\end{array}$ & 0.015 & 0.120 & & & & $0.441 * * *$ \\
\hline $\begin{array}{l}\text { Rheumatoid arthritis / collagen } \\
\text { vascular diseases }\end{array}$ & 0.006 & 0.079 & & & & $0.373 * * *$ \\
\hline Obesity & 0.014 & 0.119 & & & & $0.405^{* * *}$ \\
\hline Weight loss & 0.004 & 0.060 & & & & $1.058 * * *$ \\
\hline Blood loss anaemia & 0.004 & 0.061 & & & & $0.783 * * *$ \\
\hline Deficiency anaemias & 0.006 & 0.075 & & & & $0.344 * * *$ \\
\hline Alcohol abuse & 0.012 & 0.109 & & & & $0.168 * * *$ \\
\hline Drug abuse & 0.005 & 0.071 & & & & $0.381 * * *$ \\
\hline Psychoses & 0.003 & 0.050 & & & & $0.460 * * *$ \\
\hline Depression & 0.007 & 0.085 & & & & $0.351 * * *$ \\
\hline
\end{tabular}


Table 10: Aggregate Indicator Modelling Results (continued)

\begin{tabular}{|c|c|c|c|c|c|c|}
\hline Variables & Mean & $\begin{array}{l}\text { Std. } \\
\text { Dev. }\end{array}$ & M1 & M2 & M3 & M4 \\
\hline Constant & & & $-4.200 * * *$ & $-4.194 * * *$ & $-4.101 * * *$ & $-4.502 * * *$ \\
\hline Observations & & & $5,741,138$ & $5,741,138$ & $5,741,138$ & $5,741,138$ \\
\hline Sensitivity & & & 0.573 & 0.594 & 0.603 & 0.600 \\
\hline Specificity & & & 0.585 & 0.566 & 0.613 & 0.679 \\
\hline C-stat (Area under ROC) & & & 0.596 & 0.600 & 0.645 & 0.692 \\
\hline LR test M1 & & & & $713^{* * *}$ & $13987 * * *$ & $39586 * * *$ \\
\hline LR test $\mathrm{M} 2$ & & & & & $13274^{* * *}$ & $38873 * * *$ \\
\hline LR test M3 & & & & & & $25599 * * *$ \\
\hline
\end{tabular}

*** $p<0.01, * * p<0.05, * p<0.1$

For the aggregate indicator all 22 of the MDC categories are found to be statistically significant suggesting that relative to the reference category, MDC5 (Diseases and Disorders of the Circulatory System), the predicted probability of an AE occurring is significantly affected depending on the primary diagnosis of the patient discharge. $12 \mathrm{MDC}$ coefficients are positive and 10 are negative. These range from a low of -1.37 for MDC19 Mental Diseases and Disorders which corresponds to an odds ratio of 0.25 and a high of 0.837 , reflecting an odds ratio of 2.31 for MDC18 (Infectious and Parasitic Diseases). Similar results are found for the sub-aggregates, while for the individual PSIS MDC dummies are replaced by DRG dummies in line with the AHRQ models. The main difference between the estimation results with respect to DRGs compared to MDCs (other than that the number of DRG dummies in each model varies considerably) is that the range in magnitudes of the estimated coefficients is much wider. For example for PSI3 there are 150 DRG dummies included in the model. The coefficient estimates range from a low of -1.72 to a high of 1.96 corresponding to odds ratios of 0.18 and 7.1 respectively.

Estimated coefficients for comorbidities are typically positive and statistically significant. For the aggregate indicator, estimated coefficients are statistically significant at the five percent level for all but two of the comorbidity dummies. Of those 23 out of 25 are positive, indicating the presence of each respective comorbidity increases the predicted probability of an AE. The positive coefficient values are intuitive in that in general one would expect the presence of an additional condition would likely complicate treatment to some extent and hence increase the likelihood of an adverse event. Comorbidity coefficient estimates vary in magnitude from a low 
of -0.200 (paralysis) to a high of 1.058 (weight loss). These correspond to odds ratios of 0.819 and 2.880 indicating the predicted odds of an AE decreases by 18 percent when "paralysis" is flagged but increase nearly two-fold when weight loss is flagged. In terms of statistical significance and coefficient sign, these results are largely consistent with those of the subaggregate indicators and individual PSIs. However the magnitudes of the coefficients vary considerably.

\subsection{Empirical Evaluation of the Risk Adjustment Models}

Overall, empirical evaluation of the risk adjustment models indicates that the most appropriate risk adjustment models for each indicator are those which include all of the risk factor categories (M4). Sensitivity, specificity, and the c-statistic generally increases across the incremental models, and LR test statistics reject the nested models in favour of models containing additional risk factors. Therefore, based on these results M4 risk adjustment is preferred for the aggregate, sub-aggregate indicators (excluding obstetrics), and for PSIs 1-15. For the obstetrics sub-aggregate and PSIs17-20 M2 is preferred. Results for the aggregate indicator can be found at the bottom of Table 10. For all other indicators including the subaggregates and the individual PSIs, results can be found at the bottom of the respective regression results tables in Appendix D.

Sensitivity is the proportion of AE predicted correctly. For the aggregate indicator sensitivity increases from 0.57 (for M1) to 0.60 (for M4). This suggests that as more risk factors are added to the models their predictive power increases. Sensitivity of 0.60 means that 60 percent of adverse events are predicted correctly by the model. These results are reflected by the general sub-aggregate indicator whose sensitivity increases from 0.60 to 0.66 . However for each of the other sub-aggregates sensitivity declines across the incremental meaning the proportion of adverse events predicted correctly is declining. For example for the medical sub-aggregate sensitivity declines from 0.77 to 0.70 .

The same pattern of declining sensitivity across the incremental models is found among the individual PSIs. Sensitivity declines for 11 of the PSI (PSI3, 6, 7, 8, 9, 14, 15, 17, 18, 19, and 20). This includes all of the medical and obstetrics PSIs, and all but one of the general PSIs. 
Sensitivity increases across the models for only five indicators (PSI1, 10, 11, 12, and 13) which with the exception of PSI1 (general) are all post-operative indicators.

The results above are contrasted by specificity increasing across the incremental models for each of indicators. Specificity is the proportion of discharges not flagged with an AE that the model predicted correctly. In the case of the aggregate indicator specificity increases as risk factors are added to the models from 0.59 (for M1) to 0.68 (for M4). Therefore 68 percent of those discharges not flagged with an adverse event are predicted correctly by the model. This is reflected in all of the sub-aggregates with the general sub-aggregate increasing from 0.52 to 0.68 , medical 0.49 to 0.73 , post-operative 0.47 to 0.73 , and obstetrics 0.51 to 0.62 . This is also the case for all of the individual PSIs. The magnitudes of the specificity results for the individual PSIs are higher than the aggregate and sub-aggregates. For example specificity for PSI12 increases from 0.47 to 0.87 and for PSI15 from 0.45 to 0.89 .

The c-statistic (otherwise known as the area under the ROC curve) is a combination of sensitivity and specificity (Pencina, D'Agostino et al. 2008). It can be interpreted as the proportion of all possible pairs of discharges, one of which experiences an $A E$ and the other which does not, where the predictive probability of the discharge that experiences the $A E$ is greater than that which does not. For the aggregate indicator the c-statistic increases across each of the four incremental models. This is generally indicative of all other indicators and indicates the model's ability to discriminate improves as additional risk factors are included. More generally this means that risk adjustment becomes more accurate. For the subaggregates the c-statistic increases across each of the incremental models. For the general indicator the c-statistic increases from 0.58 to 0.73 , for medical 0.66 to 0.79 , post-operative 0.60 to 0.77 , and obstetrics 0.52 to 0.59 . Results for the individual PSIs display the same pattern: for each of the indicators the c-statistic increases for each incremental level of risk adjustment. For PSI8 the c-statistic increases from 0.70 (M1) to 0.93 (M4) and for PSI15 from $0.45(\mathrm{M} 1)$ to $0.84(\mathrm{M} 4)$.

The magnitude of the c-statistic for the M4 model for the aggregate indicator (0.69) reflects "poor" discrimination (Hosmer and Lemeshow 2000). However, with the cut-off for acceptable 
discrimination at 0.70 it is bordering on "acceptable". For the sub-aggregate indicators (excluding obstetrics) the c-statistic for M4 adjustment reflects "acceptable" discrimination. The obstetrics sub-aggregate and the individual obstetrics PSIs are all considered to have "poor" discrimination. The discrimination ability of all the other individual PSIs is generally considered to be either "acceptable" (PSI3, 6, 9, 13, 14), or "excellent" (PSI1, 10, 11, 12, and 15), with one considered "outstanding" (PSI8), and another "poor" (PSI7).

Likelihood ratio tests reject the null hypothesis that the each of the nested models is true at the $5 \%$ level of significance in favour of the models with additional risk factors. This is almost without exception for each of the sub-aggregates and individual PSIs. Of particular interest are the test results examining ethnicity and NZDep as additional risk factors. For each of the subaggregates the null model (M1) is rejected in favour of the alternative (M2) that includes ethnicity and NZDep. For the individual PSIs results are not as definitive; on three occasions (PSI1, PSI8, and PSI11) the null (M1) model is not rejected, however, in general the alternative (M2) is favoured. These results add further support to the inclusion of all risk factors in the risk adjustment models: M4 risk adjustment for the aggregate indicator, sub-aggregates (excluding obstetrics), and individual PSIs (1-15); and M2 risk adjustment for the obstetrics sub-aggregate and PSIs17-20.

\subsection{Comparison of Risk Adjusted Rates}

In this sub-section risk adjusted rates via OLS and DS are used for comparison with logistic regression based risk adjusted rates. The purpose is to attempt to assess the accuracy of logistic regression based risk adjustment when dealing with rare events.

Overall logistic risk adjusted rates correlate highly with both OLS and DS risk adjusted rates (see Table 11). For the aggregate indicator the correlation between logistic and OLS rates and subsequent rank correlation is 0.99 . The correlations with DS are lower, albeit still in excess of 0.95. The same pattern emerges with respect to the sub-aggregates; correlations with OLS are high, typically 0.99 and above, while correlations with DS are lower, but still of a relatively high magnitude (the lowest being the correlation between post-op rates at 0.87 ). 
Table 11: Comparison of Alternative Risk Adjustment Methodologies

\begin{tabular}{llllllll}
\hline PSI & Obs. & $\begin{array}{l}\text { PSI rate } \\
\text { per 1000 }\end{array}$ & $\begin{array}{l}\text { OR=0 } \\
\text { Prop. }\end{array}$ & $\begin{array}{l}\text { Logit OLS } \\
\text { RAR Corr. }\end{array}$ & $\begin{array}{l}\text { Logit DS } \\
\text { RAR Corr. }\end{array}$ & $\begin{array}{l}\text { Logit OLS } \\
\text { Rank Corr. }\end{array}$ & $\begin{array}{l}\text { Logit DS } \\
\text { Rank Corr. }\end{array}$ \\
\hline Overall & 333 & 16.44 & 0.000 & 0.994 & 0.961 & 0.996 & 0.970 \\
General & 333 & 2.63 & 0.099 & 0.998 & 0.984 & 0.997 & 0.984 \\
Medical & 333 & 11.81 & 0.000 & 0.987 & 0.893 & 0.996 & 0.935 \\
Post-op & 279 & 22.24 & 0.043 & 0.995 & 0.872 & 0.997 & 0.974 \\
Obstetric & 297 & 15.69 & 0.094 & 1.000 & 0.995 & 0.999 & 0.995 \\
\hline PSI1 & 279 & 0.01 & 0.928 & 0.989 & 0.909 & 0.447 & 1.000 \\
PSI3 & 333 & 17.49 & 0.015 & 0.979 & 0.889 & 0.995 & 0.915 \\
PSI6 & 333 & 0.37 & 0.255 & 0.998 & 0.924 & 0.986 & 0.984 \\
PSI7 & 333 & 4.94 & 0.111 & 1.000 & 0.981 & 0.997 & 0.988 \\
PSI8 & 270 & 0.46 & 0.426 & 0.982 & 0.990 & 0.936 & 0.999 \\
PSI9 & 279 & 18.78 & 0.050 & 0.996 & 0.844 & 0.998 & 0.979 \\
PSI10 & 216 & 1.8 & 0.694 & 0.995 & 0.968 & 0.812 & 0.999 \\
PSI11 & 198 & 1.42 & 0.672 & 0.996 & 0.957 & 0.831 & 0.999 \\
PSI12 & 279 & 3.19 & 0.172 & 0.988 & 0.972 & 0.994 & 0.980 \\
PSI13 & 72 & 12.8 & 0.096 & 0.999 & 0.924 & 0.998 & 0.961 \\
PSI14 & 234 & 4.7 & 0.308 & 0.992 & 0.968 & 0.970 & 0.987 \\
PSI15 & 333 & 2.23 & 0.168 & 0.999 & 0.992 & 0.994 & 0.993 \\
PSI17 & 297 & 14.99 & 0.320 & 1.000 & 0.998 & 0.976 & 1.000 \\
PSI18 & 162 & 62.69 & 0.074 & 1.000 & 0.990 & 0.999 & 0.987 \\
PSI19 & 297 & 14.36 & 0.141 & 1.000 & 0.991 & 0.996 & 0.986 \\
PSI20 & 207 & 4.06 & 0.464 & 1.000 & 0.993 & 0.941 & 0.998 \\
\hline
\end{tabular}

Results relating to the individual PSIs are generally reflective of those already discussed.

Correlations between risk adjusted rates are high, typically over 0.95 , but with three notable exceptions: PSI8, PSI13, and PSI18. Low correlations with DS risk adjusted rates can be attributed to the well-known weakness of DS; when multiple risk factors are employed, disproportionate weightings can be applied to ORs of certain strata if the observations within these strata are few. A closer inspection of the data reveals this to be the case, usually resulting in inappropriately low DS risk adjusted rates. An explanation of low correlations with OLS risk adjusted rates is more difficult. PSI8 is both a very low frequency indicator and a PSI with a relatively low at-risk population. This might explain why the correlation is poor. It could be largely due to the fact that logistic risk adjusted rate will produce risk adjusted rates equal to zero when the OR equals zero but OLS will not. It is not as clear why there is such a low correlation for PSI18. Rank correlation is high with only a few exceptions relating to OLS risk adjustment which is easily explained. Rank correlation declines between logistic and OLS risk adjusted rates as the proportion of ORs equal to zero increases (column three), and by 
derivation this is as expected. When the OR is zero logistic (and DS) risk adjusted rates will also equal zero. In contrast this is not the case for OLS based RA which will discriminate between hospitals with ORs equal to zero. As a result the correlation between both risk adjusted rates and hospital ranks will decline as the proportion of zero ORs increases ${ }^{28}$. In conclusion the logistic regression approach to risk adjustment appears robust to other standard methods.

\subsection{Results of Risk Adjustment on Hospital Level Rates}

This section shifts the focus from discharge level analysis of adverse events to analysis of hospital level rates. The purpose of analysis is two-fold: the first is to attempt to disentangle the effects of risk adjustment on hospital level ORs via the different risk factors included in the incremental models (Section 5.5.1); the second is an overall investigation of how the ranks of hospitals change from those based on ORs to the preferred M4 risk adjusted rates. This is particularly important because it assess how much of an effect risk adjustment has on relative hospital performance compared to performance measured simply of unadjusted ORs of adverse events (Section 5.5.2).

\subsubsection{Effects of Risk Adjustment On Hospital Rates Across Incremental Models}

In general risk adjustment across the incremental models has moderate effects on the distributions of hospital level rates across the indicators. The effect is greatest on the skewness of the distributions, in particular working to moderate those hospital level rates at the high end of the scale. However, these effects are not systematic across the indicators, nor are they consistent from one incremental model to the next. However, for the aggregate and subaggregate indicators some trends can be identified, particularly relating to M1 (age-gender adjustment). For the individual PSIs, results are inconsistent and any inference drawn tends to be indicator specific.

\footnotetext{
${ }^{28}$ An alternative permutation of the analysis has been conducted which sets the OLS risk adjusted rate to zero (post estimation) if the OR equals zero, and/or if the estimated OLS risk adjusted rate is negative. Rank correlations are significantly higher, particularly for those indicators with a high proportion of ORs equal to zero.
} 
Table 12 summarises the distributions of pooled annual hospital ORs and M1-M4 risk adjusted rates for each indicator: column two indicates the number of hospitals in the sample ${ }^{29}$; column three shows the mean hospital level PSI rates (per 1000 at-risk admissions); column four shows the standard deviation in the hospital level rates; and the skew statistic is displayed in the fifth column. Columns three to five are repeated for each incremental level of risk adjustment.

Standard deviation measures the concentration of annual hospital rates of adverse events around the mean. The more concentrated the rates are, the smaller the standard deviation will be. Comparing the standard deviation of rates of adverse events across the incremental models of risk adjustment permits an evaluation of how much variation is accounted for or masked by the risk factors. For example, if the standard deviation of the distribution of M1 RARs is lower than the standard deviation of ORs, this suggests that to some extent hospital level rate variation is accounted for by age and gender. An increase would suggest variation is masked by age and gender.

The skewness statistic quantifies how symmetrical the distribution is around the mean. For each of the PSIs the distributions of ORs are positively skewed indicating asymmetric distributions with long tails to the right. In other words the ORs cluster toward the lower side of the distribution (roughly at or below the mean) and there are fewer higher rates. However, those higher rates tend to be quite spread out, some with magnitudes multiple times that of the mean, hence the long right hand tail. The comparison of skewness statistics across models provides a means to interpret the effects of risk adjustment on those hospitals with high rates of adverse events at the right hand tail of the distribution. For example after M1 risk adjustment, if skewness declines, this suggests that hospitals with higher rates are moderated for by the age-gender mix of the at-risk population. Alternatively, an increase in skewness suggests differences in the age and gender casemix masks differences in hospitals with higher rates.

\footnotetext{
${ }^{29}$ Hospitals are omitted from the analysis if in any one year they do not meet the minimum denominator requirement of 30 .
} 
M1 risk adjustment reduces variation and skewness of annual hospital aggregate and subaggregate indicator rates suggesting age and gender account for variation and in particular moderate those high rates in the right hand tail. The effect on skewness is more pronounced. However, there is little consistency across the individual PSIs. For the aggregate indicator, M1 adjustment causes the standard deviation of rates to fall from 5.79 to 5.6 (a reduction of 3.5 percent). This indicates that to a certain extent variation in hospital level rates is accounted for by age and gender. Skewness statistics also decline after M1 adjustment from 0.55 to 0.42 (a 24 percent reduction), suggesting in particular higher rates are partly moderated (shifted closes to the mean) by adjusting for the age-gender mix of the at-risk population.

Figure 1: Frequency Distribution, Aggregate PSI, OR and M1 RAR

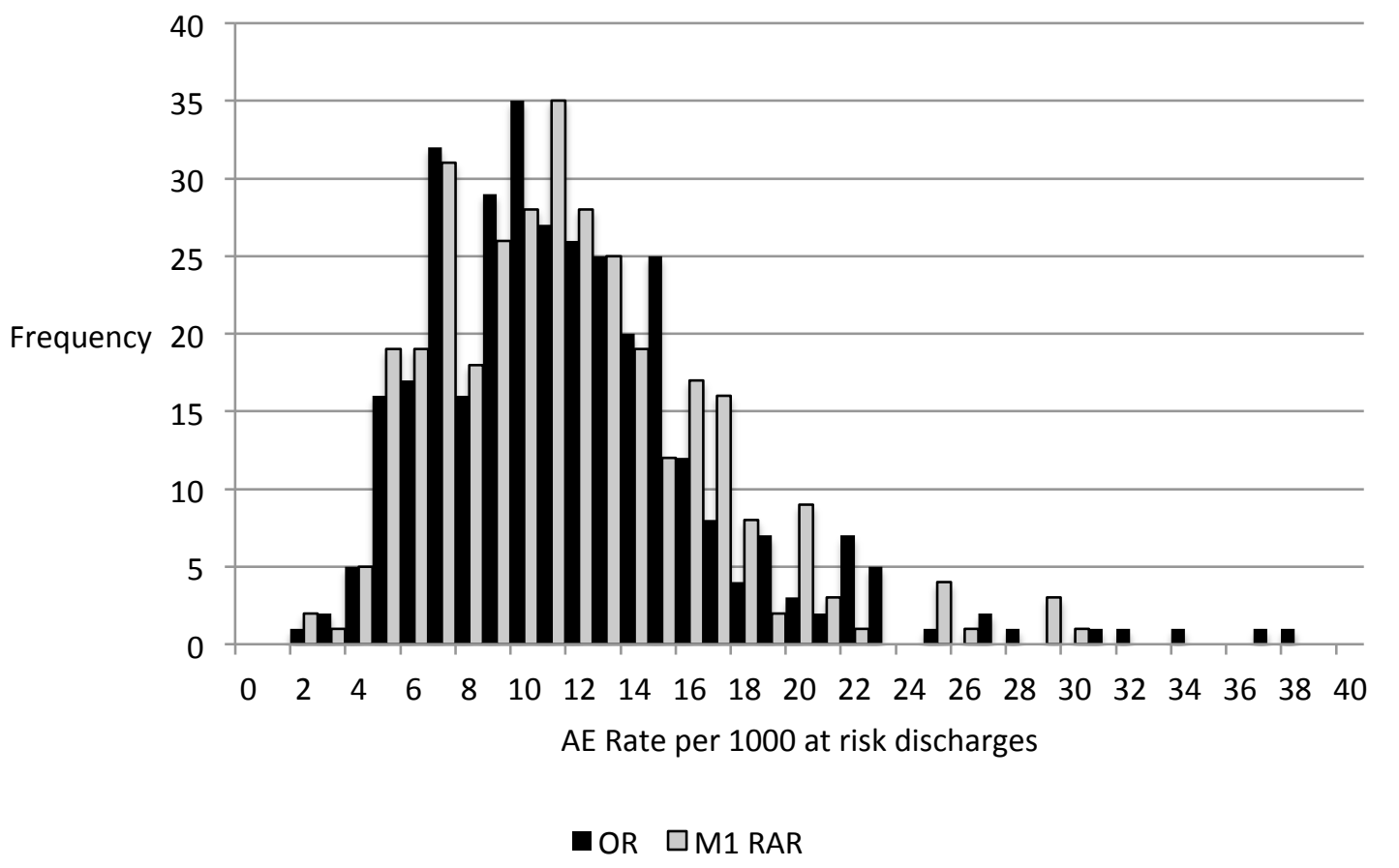

Variation and skewness also decline for the general, medical, and post sub-aggregate indicators. The effect of M1 risk adjustment on the medical sub-aggregate is the most pronounced as is shown in Figure 1. Variation in hospitals level rates declines from a standard deviation of 5.42 to 4.87 (-10 percent) and skewness from 1.47 to 0.94 (-36 percent). This is reflected in Figure 1 by a slight tightening of how much the hospital rates vary around the mean and a reduction in hospital level rates at the right tail of the distribution. For the obstetrics indicator there is no change in either variation or skewness. 
For the individual PSIs there is little consistency across the indicators with respect to M1 risk adjustment. The standard deviation and skewness of seven of the PSIs declines (PSI3, 6, 7, 9, 10, 12, and 18). For example, the standard deviation of PSI3 rates declines from 14.0 to 11.6 (17 percent) and skewness from 1.89 to 1.30 (-32 percent). In contrast three PSIs increase in variation and skewness albeit by smaller magnitudes (PSI1, 11, and 13). The standard deviation of PSI11 rates rises from 2.08 to 2.17 (4.4 percent) and skewness from 2.74 to 2.99 (9.0 percent). Two indicators, PSI8 and PSI20, display increases in variation accompanied by a reduction in skewness. The standard deviation of rates for PSI8 increases from 0.64 to 0.72 (12.5 percent) however skewness declines from 3.60 to 3.41 (-5.3 percent). A number of indicators are relatively unaffected by M1 adjustment with changes in standard deviation and skewness of one percent or less in either direction (PSI14, 15, and 19).

The effect of further adjusting for ethnicity and deprivation broadly mirrors the effects of adjusting for age and gender. In fact the magnitudes of change from $M 1$ to $M 2$ in variation and skewness is generally of a higher magnitude. For the aggregate indicator variation and skewness decline further when risk adjustment includes ethnicity and deprivation level. The standard deviation declines from 5.59 to 5.27 (-1.1 percent) and skewness from 0.42 to 0.33 (20.9 percent). This indicates variation of hospital level rates is further accounted for by socioeconomic status and ethnicity and this is particularly the case for hospitals with high rates of AEs in the right tail of the distribution. This is also reflected in each of the sub-aggregates with the exception of obstetrics whose variation and skewness increase by large magnitudes. The standard deviation of obstetrics rates increases 11.9 percent from 5.47 to 6.13 , and skewness by 40.6 percent from 0.56 to 0.79 .

Trends across the individual PSIs are again inconsistent. Six indicators exhibit the same patterns as the aggregate and majority of sub aggregate indicators with declining variation and skewness (PSI1, 3, 9, 11, 12, and 14). For example the standard deviation and skewness for PSI3 further declines by 2.1 and 2.9 percent respectively. This means the combined effect of adjustment for gender, age, ethnicity, and deprivation level results in a decrease in variation by 18.7 percent and skewness by 33.5 percent. On the other hand, variation and skewness increases for a further five PSIs (PSI6, 10, 13, 17, and 18). The results highlighted above for the 
obstetrics sub-aggregate indictor appear driven primarily by PSI17. Its standard deviation rises 24.9 percent from 6.5 to 8.2 and skewness by 29.8 percent from 2.3 to 2.9. Moving from M1 to $\mathrm{M} 2$ risk adjustment results in the variation of three indicators increasing while skewness decreases (PSI8, 19, and 20). PSI7 is relatively unchanged from the addition of adjustment for ethnicity and deprivation level.

The effects of including DRGs into risk adjustment models (M3) are different from those discussed previously. M3 adjustment results in increases to variation and skewness of the sub aggregate indicator, suggesting variation in rates is masked by diagnostic groups. This increase is driven by the general and in particular the post-operative sub-aggregate indicators ${ }^{30}$. The other noteworthy difference is in terms of magnitudes of change. For the aggregate indicator the standard deviation increases from 5.5 to 5.8 ( 3.5 percent) and skewness from 0.33 to 0.40 (20.3 percent). For the post-operative indicator variation increases by 20.9 percent and skewness increases seven fold.

Variation and skewness for the individual PSIs increases for five indicators (PSI7, 10, 11, 12, and 14). With the exception of PSI7, each of these are post-operative PSIs. The magnitudes of these increases are generally relatively higher than those presented for M1 and M2. The standard deviation of PSI12 increases from 1.72 to 1.96 (13.6 percent) and skewness increases 0.68 to 2.39 (251 percent). Four PSIs exhibit declining variation and skewness resulting from M3 risk adjustment (PSI1, 6, 9, and 13). Again the magnitudes of these changes are greater than those presented relating to $\mathrm{M} 1$ and $\mathrm{M} 2$ adjustment. For example variation in the rates of PSI decline by 10 percent and skewness falls from being positive 0.44 to negative, albeit marginally, at 0.05. Three indicators exhibit declining variation and increases in skewness (PSI3, 8, and 15).

\footnotetext{
${ }^{30} \mathrm{M} 3$ and $\mathrm{M} 4$ adjustment is not conducted on obstetrics indicators.
} 
Table 12: Risk Adjustment Results

\begin{tabular}{|c|c|c|c|c|c|c|c|c|c|c|}
\hline \multirow[b]{2}{*}{ PSI } & \multicolumn{3}{|c|}{ Observed Rate } & \multicolumn{4}{|c|}{ Age-Gender Adjusted (M1) } & \multicolumn{3}{|c|}{ Ethnicity-NZDep Adjusted (M2) } \\
\hline & $\mathbf{N}$ & Rate & SD & Skew & Rate & SD & Skew & Rate & SD & Skew \\
\hline Overall & 333 & 13.084 & 5.793 & 0.547 & 12.764 & 5.590 & 0.416 & 12.865 & 5.527 & 0.329 \\
\hline General & 333 & 1.979 & 1.317 & 0.357 & 1.966 & 1.312 & 0.354 & 1.958 & 1.293 & 0.305 \\
\hline Medical & 333 & 11.337 & 5.422 & 1.474 & 11.003 & 4.867 & 0.940 & 10.980 & 4.878 & 0.930 \\
\hline Post-op & 279 & 15.702 & 9.129 & 0.512 & 15.679 & 9.016 & 0.451 & 15.757 & 8.949 & 0.416 \\
\hline Obstetric & 297 & 8.391 & 5.474 & 0.559 & 8.388 & 5.476 & 0.562 & 9.226 & 6.125 & 0.790 \\
\hline PSI1 & 279 & 0.012 & 0.053 & 6.018 & 0.012 & 0.055 & 6.250 & 0.012 & 0.054 & 6.140 \\
\hline PSI3 & 333 & 20.178 & 14.002 & 1.890 & 19.202 & 11.627 & 1.295 & 18.989 & 11.378 & 1.257 \\
\hline PSI6 & 333 & 0.323 & 0.322 & 1.888 & 0.321 & 0.318 & 1.842 & 0.320 & 0.319 & 1.953 \\
\hline PSI7 & 333 & 4.131 & 3.723 & 1.379 & 4.109 & 3.698 & 1.358 & 4.106 & 3.688 & 1.354 \\
\hline PSI8 & 270 & 0.441 & 0.639 & 3.603 & 0.472 & 0.719 & 3.411 & 0.476 & 0.721 & 3.327 \\
\hline PSI9 & 279 & 13.116 & 7.977 & 0.537 & 13.113 & 7.885 & 0.477 & 13.192 & 7.827 & 0.437 \\
\hline PSI10 & 216 & 1.045 & 2.042 & 2.541 & 1.031 & 2.015 & 2.528 & 1.037 & 2.044 & 2.665 \\
\hline PSI11 & 198 & 1.064 & 2.075 & 2.744 & 1.082 & 2.167 & 2.992 & 1.082 & 2.154 & 2.900 \\
\hline PSI12 & 279 & 2.368 & 1.900 & 1.556 & 2.332 & 1.737 & 0.711 & 2.330 & 1.724 & 0.682 \\
\hline PSI13 & 72 & 15.903 & 13.027 & 1.167 & 16.073 & 13.376 & 1.247 & 16.248 & 13.642 & 1.269 \\
\hline PSI14 & 234 & 4.092 & 4.040 & 1.038 & 4.084 & 4.044 & 1.039 & 4.025 & 3.937 & 0.968 \\
\hline PSI15 & 333 & 1.602 & 1.258 & 0.458 & 1.597 & 1.255 & 0.466 & 1.591 & 1.231 & 0.393 \\
\hline PSI17 & 297 & 4.871 & 6.525 & 2.240 & 4.875 & 6.548 & 2.264 & 5.651 & 8.175 & 2.939 \\
\hline PSI18 & 162 & 62.157 & 38.188 & 0.560 & 61.571 & 37.652 & 0.544 & 63.324 & 38.821 & 0.583 \\
\hline PSI19 & 297 & 11.115 & 8.381 & 1.258 & 11.088 & 8.397 & 1.262 & 11.603 & 8.561 & 1.166 \\
\hline PSI20 & 207 & 2.910 & 4.600 & 3.675 & 2.946 & 4.623 & 3.570 & 2.976 & 4.652 & 3.431 \\
\hline
\end{tabular}


Table 12: Risk Adjustment Results (continued)

\begin{tabular}{|c|c|c|c|c|c|c|c|c|c|c|}
\hline \multirow[b]{2}{*}{ PSI } & \multirow[b]{2}{*}{$\mathbf{N}$} & \multicolumn{3}{|c|}{ Observed Rate } & \multicolumn{3}{|c|}{ DRG Adjusted (M3) } & \multicolumn{3}{|c|}{ Comorbidity Adjusted (M4) } \\
\hline & & Rate & SD & Skew & Rate & SD & Skew & Rate & SD & Skew \\
\hline Overall & 333 & 13.084 & 5.793 & 0.547 & 12.914 & 5.719 & 0.396 & 13.037 & 5.374 & 0.197 \\
\hline General & 333 & 1.979 & 1.317 & 0.357 & 1.977 & 1.294 & 0.409 & 1.990 & 1.309 & 0.499 \\
\hline Medical & 333 & 11.337 & 5.422 & 1.474 & 10.650 & 4.442 & 0.565 & 10.975 & 4.396 & 0.604 \\
\hline Post-op & 279 & 15.702 & 9.129 & 0.512 & 17.337 & 10.823 & 2.935 & 17.702 & 8.694 & 1.089 \\
\hline Obstetric & 297 & 8.391 & 5.474 & 0.559 & \multicolumn{6}{|c|}{ N/A } \\
\hline PSI1 & 279 & 0.012 & 0.053 & 6.018 & 0.012 & 0.053 & 5.797 & 0.012 & 0.055 & 6.297 \\
\hline PSI3 & 333 & 20.178 & 14.002 & 1.890 & 18.525 & 10.906 & 1.356 & 18.846 & 11.254 & 1.475 \\
\hline PSI6 & 333 & 0.323 & 0.322 & 1.888 & 0.310 & 0.288 & 1.448 & 0.306 & 0.274 & 1.142 \\
\hline PSI7 & 333 & 4.131 & 3.723 & 1.379 & 4.265 & 3.880 & 1.516 & 4.279 & 3.859 & 1.472 \\
\hline PSI8 & 270 & 0.441 & 0.639 & 3.603 & 0.451 & 0.670 & 3.462 & 0.495 & 0.784 & 3.797 \\
\hline PSI9 & 279 & 13.116 & 7.977 & 0.537 & 14.396 & 7.029 & -0.050 & 14.775 & 6.906 & -0.172 \\
\hline PSI10 & 216 & 1.045 & 2.042 & 2.541 & 1.056 & 2.172 & 3.048 & 1.039 & 2.428 & 4.760 \\
\hline PSI11 & 198 & 1.064 & 2.075 & 2.744 & 1.087 & 2.271 & 3.612 & 1.075 & 2.429 & 4.588 \\
\hline PSI12 & 279 & 2.368 & 1.900 & 1.556 & 2.446 & 1.959 & 2.394 & 2.663 & 2.833 & 8.462 \\
\hline PSI13 & 72 & 15.903 & 13.027 & 1.167 & 14.896 & 10.921 & 0.862 & 14.850 & 10.945 & 0.903 \\
\hline PSI14 & 234 & 4.092 & 4.040 & 1.038 & 4.034 & 3.985 & 1.074 & 4.151 & 4.265 & 1.362 \\
\hline PSI15 & 333 & 1.602 & 1.258 & 0.458 & 1.704 & 1.216 & 0.449 & 1.709 & 1.223 & 0.483 \\
\hline PSI17 & 297 & 4.871 & 6.525 & 2.240 & & & & & & \\
\hline PSI18 & 162 & 62.157 & 38.188 & 0.560 & \multirow{3}{*}{\multicolumn{6}{|c|}{ N/A }} \\
\hline PSI19 & 297 & 11.115 & 8.381 & 1.258 & & & & & & \\
\hline PSI20 & 207 & 2.910 & 4.600 & 3.675 & & & & & & \\
\hline
\end{tabular}


M4 adjustment for comorbidities offers some contrasting results to those already discussed. Unlike the effects of $\mathrm{M} 1, \mathrm{M} 2$, and $\mathrm{M} 3$ adjustment, there is little consistency within the aggregate and sub-aggregate indicators in terms of the distribution change of hospital rates. However, for the individual PSIs, the effects of adjustment are largely consistent across the indictors. For the aggregate indicator variation and skewness decline by 6.0 and 50.3 percent respectively. This appears to be driven by declines in variation and skewness of the post-operative sub-aggregate indicator of 19.7 percent and 62.9 percent. On the other hand variation and skewness increase for the general sub-aggregate indicator and for the medical sub-aggregate variation decreases marginally while skewness increases.

For the individual PSIs, adjustment for comorbidities increases both variation and skewness (PSI1, 3, 8, 10, 11, 12, 13, 14, and 15). As with adjustment for DRGs the magnitudes can be large at times. The standard deviation for PSI12 for example increases from 1.96 to 2.83 (44.6 percent) and skewness from 2.39 to 8.46 (253 percent). Only rates reflected by PSIs 6 and 7 decrease in variation and skewness.

\subsubsection{Effects of M4 Risk Adjustment on Hospital Ranks}

This section investigates the effects of risk adjustment on relative hospital performance. Comparisons are made between hospital rankings based on unadjusted ORs and those based on M4 risk adjusted rates, the preferred risk adjustment model. Unlike the previous section, the intent is not to disentangle the effects of the various risk factors, rather it is to understand the extent of the overall impact of risk adjustment on relative hospital performance.

Another difference is that hospital rates are not calculated annually, they are calculated using pooled discharge data across the nine years of the study period. Therefore observed and risk adjusted rates for each hospital equate to their overall rate of adverse events from 2001 to 2009 . For each PSI the maximum number of hospitals is 37 , however for many indicators hospitals have been dropped from the analysis because their at-risk population is fewer than 30. Those which are impacted most by this are the surgical and obstetrics indicators and this is due to the operational capacity of those hospitals. The resulting range 
of hospitals ranked within each indicator is from a low of 26 for PSI18 to the maximum of 37 for the aggregate indicator, general and medical sub-aggregates, and PSI3, 6, and 7.

The analysis examines five alternative metrics to gauge the effect of risk adjustment on hospital rankings which are presented in Table 13. The first two look at the overall change in hospital rankings using the Spearman rank correlation coefficient, $\rho$ (column three) and the mean absolute change in hospital rankings (column four). Namely, hospitals are ranked from first to last by way of their ORs and then again by their RARs. The Spearman rank correlation coefficient is the Pearson correlation between the newly created rank variables. For the mean absolute change, the difference between OR ranking and RAR ranking for each hospital is calculated. For example, if hospital A is ranked third by way of ORs and then eighth after risk adjustment the difference in rank is negative five. The absolute value of that difference is taken, and then the combined difference in ranks across all hospitals is taken. The change in performance of the best (and worst) performers is measured by the percentage of hospitals that remain in the top (and bottom) 20 percent after M4 adjustment (columns five and six). The change in rankings across the distribution is measured by the percent of hospitals whose ranking shifts across two or more deciles after risk adjustment (column seven).

The rank correlation statistic measures the way relative hospital performance is impacted by risk adjustment. In general the results from this analysis suggest that risk adjustment has very little effect on relative hospital rankings. The rank correlation coefficient for the aggregate indicator is 0.89 which indicates a positive and high correlation between the observed and risk adjusted rates. This is generally reflective of the other indicators. Of the sub-aggregates the indicator affected the least by risk adjustment is obstetrics $(\rho=0.96)$ followed closely by general $(\rho=0.94)$. In contrast the medical and post-op sub-aggregates are affected more $(\rho=0.80)$. The individual PSIs are affected less on average. Their respective rank correlations are all greater than 0.9 with the exception of PSI3 $(\rho=0.83)$. Those indicators affected least by risk adjustment in this sense are PSI1, PSI7, PSI10, PSI11, PSI18, and PSI20 with rank correlation coefficients upward of 0.99 . 
The mean absolute change in hospital rank resulting from risk adjustment is another measure of the relative importance of risk adjustment. Rankings change on average by three places for the aggregate indicator. For the sub-aggregates this ranges from 1.8 (general) to 4.5 (medical). Results are more variable with respect to the individual PSIs. The mean absolute change in hospital rank is less than one for several indicators (PSI1, 10, 11, 18 , and 20). For the majority of the indicators the rank change falls into the 1-3 range (PSI6, $7,8,9,12,13,14,15,17$, and 19). The maximum is 4.1 (PSI3) which is consistent with its rank correlation result. However, overall these results seem in contrast to the rank correlation results. Those suggest adjusted and unadjusted hospital rankings are highly correlated, while this analysis has shown hospital rankings on average change by several positions.

Table 13: Risk Adjustment Results 2

\begin{tabular}{lllllll}
\hline PSI & N & Rank Corr. & Abs. Value & Top 20\% & Bottom 20\% & Two Declines \\
\hline Overall & 37 & 0.885 & 2.973 & 0.857 & 0.714 & 0.054 \\
General & 37 & 0.941 & 1.838 & 0.857 & 0.857 & 0.027 \\
Medical & 37 & 0.796 & 4.541 & 0.857 & 0.714 & 0.216 \\
Post-op & 35 & 0.798 & 3.029 & 0.714 & 0.429 & 0.086 \\
Obstetric & 35 & 0.959 & 2.000 & 0.714 & 0.714 & 0.029 \\
\hline PSI1 & 35 & 0.997 & 0.514 & 1.000 & 0.857 & 0.000 \\
PSI3 & 37 & 0.832 & 4.054 & 0.571 & 0.857 & 0.162 \\
PSI6 & 37 & 0.961 & 2.432 & 1.000 & 0.714 & 0.000 \\
PSI7 & 37 & 0.986 & 1.243 & 0.714 & 0.857 & 0.000 \\
PSI8 & 34 & 0.912 & 2.765 & 0.857 & 0.571 & 0.059 \\
PSI9 & 35 & 0.908 & 2.800 & 0.857 & 0.714 & 0.057 \\
PSI10 & 31 & 0.995 & 0.581 & 1.000 & 0.833 & 0.000 \\
PSI11 & 31 & 0.995 & 0.516 & 1.000 & 0.833 & 0.000 \\
PSI12 & 35 & 0.923 & 2.629 & 0.857 & 0.857 & 0.086 \\
PSI13 & 28 & 0.977 & 1.286 & 0.833 & 0.833 & 0.000 \\
PSI14 & 27 & 0.968 & 1.333 & 0.800 & 0.800 & 0.037 \\
PSI15 & 37 & 0.928 & 2.865 & 1.000 & 0.857 & 0.162 \\
PSI17 & 35 & 0.980 & 1.257 & 0.857 & 0.857 & 0.000 \\
PSI18 & 26 & 0.995 & 0.231 & 1.000 & 0.800 & 0.000 \\
PSI19 & 35 & 0.964 & 1.886 & 0.714 & 0.857 & 0.029 \\
PSI20 & 25 & 0.994 & 0.480 & 1.000 & 0.800 & 0.000 \\
\hline & & & & & & \\
\hline
\end{tabular}

The percentage of hospitals remaining in the top (and bottom) quintile of the ranking scale after risk adjustment is relatively high, although change is greater for the bottom quintile. 
For the aggregate indicator 86 percent of hospitals remain in the top quintile after risk adjustment compared to 71 percent that remain in the bottom. For the sub-aggregates between 71 percent and 86 percent remain in the top quintile, however as low as 43 percent (post-operative) remain in the bottom. This suggests risk adjustment has the greatest effect on the poorest performing hospitals.

The same results with respect to the effect of risk adjustment on the best and worst performing hospitals exists within the individual PSIs. However the percentage of hospitals remaining in the top quintile after adjustment is 100 percent for seven of the indicators (PSI1, 6, 10, 11, 15, 18, and 20). The corresponding percentage of hospitals remaining in the bottom quintile after adjustment for those same PSIs ranges from 71 percent (PSI6) to 86 percent (PSI1 and 15). There are three exceptions whereby the percentage of hospitals remaining in the top quintile after adjustment is less than those remaining in the bottom (PSI3, 7, and 19). For example, only 57 percent of hospitals remain in the bottom quintile after adjustment for PSI3 while 86 percent remain in the bottom.

The final measure, the percentage of hospitals whose position in the distribution changes by two deciles or more, suggests relatively few hospitals have their ranking significantly changed due to risk adjustment. At the aggregate level, five percent of hospitals are affected in this way. For the sub-aggregates this number is relatively low for general, postoperative, and obstetrics $(2.7,8.6$, and 2.9 percent respectively). However for the medical sub-aggregate 22 percent of hospitals change in rank by two or more deciles. For the individual PSIs results suggest the impact of risk adjustment is less. More than half have no hospitals whose position in the distribution changes by two or more deciles (PSI 1, 6, 7, 10, $11,13,17,18$, and 20). Only two, PSI3 and PSI15 (both 16 percent), have more than 10 percent of hospitals that are affected in this way. 


\section{Section 6 Discussion}

This chapter reflects on the main findings of the research. It does so by interpreting the results from Section 5, relating these back to the literature, and highlighting where this research has made its own contributions.

Section 6.1 begins by reflecting on the rationale for exploring the AHRQ PSIs applied to New Zealand data, in particular focussing on their strengths and weaknesses. Section 6.2 summarises and interprets the main findings from the descriptive analysis conducted on the AHJRQ PSIs. Section 6.3 provides a comprehensive discussion relating to the results of risk adjustment. In particular it reflects on those results and attempts to bring clarity to their meaning and relevance. Section 6.4 provides a summary of the limitations of the study and discusses possible future work. Section 6.5 provides a short conclusion.

\subsection{Measuring Patient Safety}

Since the seminal publication To Err is Human: Building a safer health care system highlighted the extent of medical error in health care, patient safety and quality of care have become a priority worldwide and New Zealand is no exception (Kohn, J. M.Corrigan et al. 2000). This thesis aims to develop patient safety measures for New Zealand public hospitals using the AHRQ PSIs. The advantages of the AHRQ PSIs are that these measures can be derived at low cost from hospital administrative data periodically and systematically. They are reflective of quality of health care services along multiple dimensions, and risk adjusted so comparable across providers and over time.

However these advantages need to be moderated with awareness of their limitations. The major concern with the use of the PSIs is their reliance on administrative data and the credibility of the limited clinical detail they include (Zhan C and Miller MR 2003). As a result, statistical indicators can have low sensitivity and specificity (Bates, O'Neil et al. 1995, West, Weeks et al. 2008). In addition, any identification of medical error is reliant on the accuracy of the ICD-10 coding system. It is generally considered that principal diagnoses are well 
recorded, but less confidence exists about the ability of administrative datasets to accurately account for complications and comorbidities (Quan, Sundararajan et al. 2005).

The research suggests that the strengths of the PSIs are such that their use needs to be further developed in New Zealand as a measure of hospital safety and quality that can complement alternative measures currently being pursued.

\subsection{AHRQ PSIs Applied to New Zealand Data}

Using the ARHQ PSIs, LOS and cost of care are both found to increase for discharges when an adverse event is flagged. While the calculations made in this study are intended as a guide only, the subsequent results are remarkably similar, and lend support to the large body of literature which highlights the economic cost of medical error discussed in Section 2 (Johnson WG, Brennan TA et al. 1992, Thomas, Studdert et al. 1999, Vincent, Neale et al. 2001, Zhan C and Miller M 2003). The findings indicate that on average AEs result in ALOS increasing by eight days. This compares to nine days concluded in a previous New Zealand study (Davis P, Lay-Yee R et al. 2001). Results also suggest that cost of care increases by around $\$ 12,000$ (in 2011/12 dollars) due to medical error. Similarly increased cost of care is found to be $\$ 10,264$ (in 2002 dollars) by an earlier New Zealand study (Brown, McArthur et al. 2002). Overall these results reinforce the importance of patient safety and quality in the health sector through providing more evidence of the economic implications of medical error.

Descriptive analysis on the PSIs investigating denominators, numerators, and corresponding rates of adverse events produces results which are broadly in line with the similar study conducted by Hider et al. 2014. There are small differences across each of the indicators but these can largely be attributed to the fact that Hider et al. do not state the use of any filters in their study. Importantly the descriptive analysis shows that some indicators are hindered by infrequent rates, prompting alternative options such as the aggregate and subaggregate PSIs. In particular PSI1: Complications of anaesthesia, PSI5: Foreign body left in during procedure, and PSI16: Transfusion reaction, are found to have rates of less than 0.1 
per 1000 at-risk patients. These findings are consistent with the Hider et al. study and also consistent with the AHRQ's empirical analysis (McDonald, Romano et al. 2002).

Comprehensive descriptive analysis investigating the relationship between potential risk factors and the PSIs demonstrates a strong empirical case for risk adjustment. Raw rates of adverse events typically show statistically significant associations with gender, age, ethnicity, NZDep, MDCs, and the presence of comorbidities. The strongest associations suggest that the incidence of AEs increases with age, and the presence of comorbidities. These findings support the pragmatic approach of employing the existing AHRQ risk adjustment models and suggest the importance of including New Zealand specific risk factors, ethnicity and NZDep. This is the first time such analysis into the risk factors associated with the PSIs has been conducted on New Zealand data.

\subsection{Risk Adjustment Results}

Logistic regression based risk adjustment has been used to construct comparable hospital measures of patient safety. Logistic regression results across all indicators generally find statistically significant coefficients for each set of risk factors. Most notably, the predicted probabilities of adverse events systematically increase with age, a trend apparent across all indicators. This result is in line with the AHRQ logistic regression results for comparable models (AHRQ 2007).

Empirical evaluation of the risk adjustment models suggests that the more complex models (M4), those including the most risk factors, are most appropriate. While sensitivity typically declines across the incremental models, specificity increases by larger magnitudes. As a result c-statistics across all models and all indicators also improve. These results are reinforced by likelihood ratio tests results which reject nested models in favour of models with additional risk factors. They reflect those found by the AHRQ in their risk adjustment modelling and empirical review (McDonald, Romano et al. 2002). The c-statistic results also offer another valuable insight into the PSIs. Hosmer and Lemeshow categorise the discriminatory power of models into poor, acceptable, excellent, and outstanding based on c-statistic results (Hosmer and Lemeshow 2000). In this research PSI7 and each of the 
obstetrics PSIs, including the obstetrics sub-aggregate, are considered "poor". This suggests that the usefulness of the obstetrics indicators in particular may be limited.

Analysis investigating the effect of risk adjustment on hospital level rates shows only a minimal effect. The distributions of hospital rates are affected in inconsistent ways by the different sets of risk factors contained in the incremental models. Age, gender, ethnicity, and deprivation tend to reduce the variation and skewness of the distribution of hospital rates. On the other hand adjustment for diagnosis categories causes increases in variation and skewness. Adjustment for comorbidities does not demonstrate any clear pattern across the indicators. Despite these inconsistencies, the effect on distribution skewness is the greatest. This suggests that while risk adjustment might not be so important across all of the hospitals, it certainly seems to have an impact on those hospitals with high relative rates.

The impact of risk adjustment on relative hospital performance is also generally small. Strong positive correlations between hospital ranks based on raw and M4 risk adjusted rates suggest a minimal effect. However, this is contrasted somewhat by the finding that the absolute change in hospital rank due to risk adjustment on average ranges from one to three places. Furthermore, the effect on those hospitals ranked in the bottom 20 percent is high. This reinforces the finding highlighted above and suggests that while risk adjustment does not have a major impact on relative hospital performance, the impact is not negligible and in particular those hospitals which ranked poorly based on ORs are impacted on the most.

Several questions are therefore raised: Why might risk adjustment not be showing huge effects? Are there any underlying issues which might be prohibiting the models from working effectively? And, given risk adjustment seems to make little difference, does risk adjustment matter?

It could of course be entirely plausible that the risk adjustment approach is working exactly as it is intended, and the minor change to the relative performance of hospitals is simply 
the reality. This highlights a major challenge when trying to assess the effectiveness of risk adjustment: one simply does not know what the so-called "true" quality measure is. Therefore the fact that risk adjustment generally does not have large effects on hospital rankings might not be an issue.

Alternatively, several issues with the risk adjustment process may potentially explain why its impact is small. A major concern with the AHRQ PSIs is they flag potential adverse events; they are not markers of actual adverse events. As a result the accuracy of the indicators could be in question. The indicators have to some extent been validated overseas but some have questioned how accurate they really are. Furthermore the indicators have not been validated on New Zealand data so it is not known how accurate they are. In addition, any identification of medical error is reliant on the accuracy of the clinical coding system. Without present on admission flags in NMDS data it is difficult to accurately account for complications and comorbidities. As a result the accuracy of the indicators might be further called into question.

Another potential issue is that logistic regression with rare events can results in bias (King and Zeng 2001). Some PSIs in particular identify AEs which are highly infrequent. As a result risk adjustment results for some indicators may suffer from bias which might in turn may be minimising the overall effect of risk adjustment on hospital level rates.

Finally, the issue of reliability which was broached in the introduction might be having an impact. McClellan and Staiger suggest that observed measures are a combination of signal and noise (McClellan and Staiger 2000). They propose methods which isolate the true quality measure from the noise. This issue has not been addressed in the current study and as a result could be impacting on the results.

Given all of this, does risk adjustment actually matter? First and foremost, while the effect of risk adjustment is not great, there is an effect none the less. To dismiss the issue would be a mistake. Furthermore, a major criticism of hospital profiling is that the measures used often neglect to address differing casemix across hospitals. As a result hospitals with poor 
rank might understandably point to the fact that their casemix is unfavourable compared to their counterpart and suggest that this, and not quality of care, is the reason for their poor relative performance. More generally, measures without risk adjustment can often be dismissed as meaningless by key stakeholders. With this in mind a major advantage of logistic regression based risk adjustment is that it is a well-known and well-understood method of addressing casemix variation within the healthcare profession. Unlike other more complex models it is not perceived as a black box which cannot be understood. As a result there will generally be fewer criticisms from within the health sector of the logistic regression based approach to risk adjustment.

\subsection{Limitations of current work and possibilities for future work}

As discussed, one of the biggest gaps in this research is to know exactly how well the AHRQ PSIs fare when used with New Zealand data. It is understood that research validating the AHRQ PSIs based on New Zealand data is currently in progress and preliminary findings appear positive, but until this knowledge gap is filled there will continue to be a question mark over the use of the AHRQ PSIs in New Zealand.

The issue of reliability is another areas that could be pursued further. Various methods have been proposed to adjust health care quality measures for reliability including: shrinkage estimators (Stein 1956), hierarchical models (Dimick, Staiger et al. 2010), empirical Bayes models (Thomas, Longford et al. 1994), and the econometric methodology proposed by McCellan and Staiger.

Another possibility for future research would be to work towards alternative aggregations of the PSIs. The AHRQ have proposed several composite indicators based on their PSIs (AHRQ 2008). These combine the individual indicators into a single quality measure by using various weighting systems: Principal Components Analysis (PCA), denominator weights, or numerator weights. An alternative could also be weights developed from the perceived importance of each PSI in measuring patient safety. One disadvantage with aggregating indicators into a single patient safety/quality measure is that, because the PSIs measure different dimensions of safety, it is possible and indeed probable that some 
indicators will be negatively correlated. Hence a PCA based approach might most appropriate.

A final avenue for future research is based on a recent development in data availability in New Zealand. The Integrated Data Infrastructure (IDI) is a large database containing microdata about people and households developed by Statistics New Zealand which links data from a range of government agencies and Statistics New Zealand surveys (Statistics New Zealand 2016). The present study was restricted to the use of NMDS only, however the IDI would permit the linkage of NMDS to a range of other data sets and permit additional risk factors such as health behaviours and hazardous drinking to be incorporated.

\subsection{Conclusion}

This research has contributed to the literature by developing the knowledge base around the AHRQ PSIs, specifically with respect to their application to New Zealand data. It has developed a robust New Zealand specific risk adjustment methodology, utilising existing AHRQ risk adjustment models, tailored to the New Zealand environment by including ethnicity and deprivation as additional risk factors. The risk adjustment models have been evaluated and their respective results analysed. As a result the research takes us a step closer to being able to confidently measure patient safety and quality of care in New Zealand.

Findings suggest that the application of the AHRQ PSIs to New Zealand data should incorporate risk adjustment particularly if the purpose is to report on comparative patient safety and quality measures. More specifically, logistic regression results indicate that each of the risk factors modelled tends to improve the predictive ability of the models. Empirical evaluation statistics confirm that the most appropriate risk adjustment models are those which include all of the risk factors modelled: gender, age, ethnicity, deprivation level, DRGs, and comorbidities. Overall the impact of risk adjustment is relatively small, however there is some impact and it must be acknowledged that that is important. Specifically, risk adjustment has the greatest impact on those hospitals with poor rankings suggesting that the impact of risk adjustment is particularly relevant in this case. 
A limitation of the research stems from the nature of the PSIs. The indicators do not necessarily indicate adverse events. Rather, they are predictors of adverse events based on the information contained within each event in the hospital administrative data. How accurate they are in predicting adverse events is not currently known, and will not be until sufficient research has been conducted into their validity with New Zealand data.

Nonetheless, this research has developed new measures of patient safety in New Zealand which are low cost, comprehensive, unobtrusive, cover multiple dimensions of patient safety, and in theory are comparable both across hospitals and over time. Further work is required but this research has developed a strong platform to build on. 


\section{Bibliography}

Adler, L., et al. (2008). "Global trigger tool: implementation basics." Journal of Patient Safety 4(4): 245-249.

AHRQ (2003). AHRQ's Patient Safety Initiative: Building Foundations, Reducing Risk. Interim Report to the Senate Committee on Appropriations. . Rockville, MD, AHRQ.

AHRQ (2006). Guide to Patient Safety Indicators. AHRQ Quality Indicators, AHRQ.

AHRQ (2007). Guide to Patient Safety Indicators. AHRQ Quality Indicators, AHRQ.

AHRQ (2008). Patient Safety Indicators (PSI) Composite Measure Workgroup Final Report., AHRQ.

AHRQ (2011). Quality Indicator Empirical Methods.

AHRQ (2014). "Voluntary Patient Safety Event Reporting (Incident Reporting) " Patient Safety Primers. from http://psnet.ahrq.gov/primer.aspx?primerID=13.

American Hospital Association (1999). Hospital Statistics. Chicago.

Anderson, R. N. and N. C. f. H. Statistics (2003). Deaths: leading causes for 2001, Citeseer.

Aspden, P., et al. (2004). Patient safety: achieving a new standard for care, National Academies Press.

ASQHC (2010). Australian Safety and Quality Framework for Health Care. Sydney, Australian Commission on Safety and Quality in Health Care.

Baker, G. R., et al. (2004). "The Canadian Adverse Events Study: the incidence of adverse events among hospital patients in Canada. ." CMAJ Canadian Medical Association Journal Can Med Assoc. 170(11): 1678-1686.

Bates, D. W., et al. (1995). "Evaluation of screening criteria for adverse events in medical patients." Medical Care 33(5): 452-462. 
Beckmann, U., et al. (1996). "The Australian Incident Monitoring Study in Intensive Care: AIMS-ICU. The development and evaluation of an incident reporting system in intensive care." Anaesthesia and intensive care 24(3): 314-319.

Bismark, M., et al. (2006). "Relationship between complaints and quality of care in New Zealand: a descriptive analysis of complainants and non-complainants following adverse events." Quality and Safety in Health Care 15(1): 17-22.

Blumenthal, D. (1996). "Quality of Care - What is It?" New England Journal of Medicine 335(12): 891-894.

Bottle, A. and P. Aylin (2009). "Application of AHRQ patient safety indicators to English hospital data." Quality and Safety in Health Care 18(4): 303-308.

Brennan, T. A., et al. (1991). "Incidence of Adverse Events and Negligence in Hospitalized Patients." New England Journal of Medicine 324(6): 370-376.

Breslow, N. E. and N. Day (1975). "Indirect standardization and multiplicative models for rates, with reference to the age adjustment of cancer incidence and relative frequency data." Journal of Chronic Diseases 28(5-6): 289-303.

Brown, P., et al. (2002). "Cost of medical injury in New Zealand: a retrospective cohort study." Journal of Health Services Research \& Policy 7(suppl 1): 29-34.

Buchner, F. and J. Wasem (2003). "Needs for further improvement: risk adjustment in the German health insurance system." Health policy 65(1): 21-35.

Carey, K. and T. Stefos (2011). "Controlling for quality in the hospital cost function." Health Care Management Science 14(2): 125-134.

Centers for Medicare and Medicaid Services. "CMS Hospital Compare Website." Retrieved October 26th, 2014, from http://www.medicare.gov/hospitalcompare/search.html.

Cevasco, M., et al. (2011). "Validity of the AHRQ Patient Safety Indicator "Central Venous Catheter-Related Bloodstream Infections"." Journal of the American College of Surgeons 212(6): 984-990. 
Chassin, M. R. and R. W. Galvin (1998). "The Urgent Need to Improve Health Care Quality." JAMA: The Journal of the American Medical Association 280(11): 1000-1005.

Chen, Q., et al. (2011). "Detecting patient safety indicators: how valid is "foreign body left during procedure" in the Veterans Health Administration?" Journal of the American College of Surgeons 212(6): 977-983.

Chertow, G., et al. (2006). "Mortality after acute renal failure: models for prognostic stratification and risk adjustment." Kidney international 70(6): 1120-1126.

Christiaans-Dingelhoff, l., et al. (2011). "To what extent are adverse events found in patient records reported by patients and healthcare professionals via complaints, claims and incident reports?" BMC health services research 11(1): 1.

Christiansen C and Morris C (1997). "Improving the statistical approach to health care provider profiling." Ann Intern Med 127 (8 pt 2): 764-768.

Classen, D. C., et al. (1991). "Computerized surveillance of adverse drug events in hospital patients." JAMA 266(20): 2847-2851.

Classen, D. C., et al. (2011). "'Global trigger tool'shows that adverse events in hospitals may be ten times greater than previously measured." Health Affairs 30(4): 581-589.

Coffey, R. M., et al. (2005). "Racial, ethnic, and socioeconomic disparities in estimates of AHRQ patient safety indicators." Medical Care 43(3): I-48-I-57.

Cohen, M. E., et al. (2009). "Risk adjustment in the American College of Surgeons National Surgical Quality Improvement Program: a comparison of logistic versus hierarchical modeling." Journal of the American College of Surgeons 209(6): 687-693.

Corrigan JM, et al. (2001). Crossing the Quality Chasm: A New Health System for the 21st Century. Washington DC, National Academy Press.

Cullen, D. J., et al. (1995). "The incident reporting system does not detect adverse drug events: a problem for quality improvement." The Joint Commission journal on quality improvement 21(10): 541-548.

Darzi, A. (2008). "Quality and the NHS Next Stage Review." The Lancet 371(9624): 15631564. 
Davies S, et al. (2001). Refinement of the HCUP Quality Indicators, Technical Reviews, No. 4, Rockville, Md: Agency for Healthcare Research and Quality.

Davis P, et al. (2001). Adverse Events in New Zealand Public Hospitals: Principal Findings from a National Survey., Ministry of Health. Occasional Paper No. 3.

DeLong, E. R., et al. (1997). "Comparing risk-adjustment methods for provider profiling." Statistics in Medicine 16(23): 2645-2664.

Deming, W. E. (1986). "Out of Crisis, Centre for Advanced Engineering Study." Massachusetts Institute of Technology, Cambridge, MA.

Department of Health (2008). High Quality Care for All: NHS Next Stage Review Final Report. London.

Department of Health and Human Services (2009). "Patient Safety Indicators." from http://www.health.vic.gov.au/psi/auspsi/development-process.

Dimick, J. B., et al. (2010). "Risk adjustment for comparing hospital quality with surgery: how many variables are needed?" Journal of the American College of Surgeons 210(4): 503508.

Dimick, J. B., et al. (2010). "Ranking Hospitals on Surgical Mortality: The Importance of Reliability Adjustment." Health Services Research 45(6p1): 1614-1629.

Donabedian, A. (1966). "Evaluating the Quality of Medical Care." The Milbank Memorial Fund Quarterly 44(3): 166-206.

Donabedian, A. (1980). "The Definition of Quality and Approaches to Its Assessment, Vol. I: Explorations in Quality Assessment and Monitoring." Ann Arbor: Health Administration Press.

Donabedian, A. (1985). Explorations in quality assessment and monitoring Vol 3 The methods and findings of quality assessment and monitoring: an illustrated analysis, Ann Arbor: Health Administration Press.

Doupi, P., et al. (2013). "IHI Global Trigger Tool and patient safety monitoring in Finnish hospitals-Current experiences and future trends." Raportti: 2013_019. 
Elixhauser, A., et al. (1998). "Comorbidity Measures for Use with Administrative Data." Medical Care 36(1): 8-27.

Emanuel, L., et al. (2008). "What exactly is patient safety?".

Franklin, B. D., et al. (2010). "Testing a trigger tool as a method of detecting harm from medication errors in a UK hospital: a pilot study." International Journal of Pharmacy Practice 18(5): 305-311.

Fuchshuber, P. R., et al. (2012). "The power of the National Surgical Quality Improvement Program - achieving a zero pneumonia rate in general surgery patients." Perm J 16(1): 3945.

Gatsonis, C., et al. (1993). "Geographic variation of procedure utilization: a hierarchical model approach." Medical Care: YS54-YS59.

Good, V., et al. (2011). "Large-scale deployment of the Global Trigger Tool across a large hospital system: refinements for the characterisation of adverse events to support patient safety learning opportunities." BMJ quality \& safety 20(1): 25-30.

Hanley, J. A. and B. J. McNeil (1982). "The meaning and use of the area under a receiver operating characteristic (ROC) curve." Radiology 143(1): 29-36.

Hannan, E. L., et al. (1994). "Improving the outcomes of coronary artery bypass surgery in New York State." JAMA 271(10): 761-766.

Haraden, C. and J. Leitch (2011). "Scotland's successful national approach to improving patient safety in acute care." Health Affairs 30(4): 755-763.

Harrell, F. E. (2001). Regression modeling strategies: with applications to linear models, logistic regression, and survival analysis, Springer.

Hayward RA and Hofer TP (2001). "Estimating Hospital Deaths Due to Medical Errors: Preventability Is in the Eye of the Reviewer." JAMA 286(4): 415-420.

Health Quality \& Safety Commission (2012). The Global Trigger Tool: A Practical Implementation Guide for New Zealand District Health Boards 
. Wellington, Health Quality \& Safety Commission.

Health Quality \& Safety Commission (2012). New Zealand Health and Disability Services National Reportable Events Policy 2012. Wellington, Health Quality \& Safety Commission.

Health Quality \& Safety Commission (2013). Global Trigger Tools: A Review of the Evidence. Wellington: Health Quality \& Safety Commission.

Health Quality \& Safety Commission New Zealand (2013). Statement of Intent 2013 to 2016. Wellington, Health Quality \& Safety Commission New Zealand.

Health Services Research and Development Service (1997). Risk Adjustment: A Tool for Leveling the Playing Field.

Hider, P., et al. (2014). "Can patient safety indicators monitor medical and surgical care at New Zealand public hospitals?".

Hosmer, D. W. and S. Lemeshow (2000). "Introduction to the logistic regression model." Applied Logistic Regression, Second Edition: 1-30.

Iezzoni, L. I. (1997). "Assessing quality using administrative data." Annals of internal medicine 127(8_Part_2): 666-674.

lezzoni, L. I. (2003). Risk adjustment for measuring health care outcomes, Chicago: Health Asministration Press.

lezzoni LI, et al. (1992). "A method for screening the quality of hospital care using administrative data: preliminary validation results." QRB Qual Rev Bull 18(11): 361-371.

Jenkins, K. J., et al. (2002). "Consensus-based method for risk adjustment for surgery for congenital heart disease." The Journal of thoracic and cardiovascular surgery 123(1): 110118.

Jha, A. K. and A. M. Epstein (2006). "The predictive accuracy of the New York State coronary artery bypass surgery report-card system." Health Affairs 25(3): 844-855. 
Johnson WG, et al. (1992). "The economic consequences of medical injuries: Implications for a No-fault Insurance Plan." Journal of the American Medical Association 267(18): 24872492.

Joint Commission Resources (2011). Benchmarking in Health Care, Joint Commission Resources.

Kaafarani, H. M. A., et al. (2011). "Validity of Selected Patient Safety Indicators:

Opportunities and Concerns." Journal of the American College of Surgeons 212(6): 924-934.

Khuri, S. F., et al. (1997). "Risk adjustment of the postoperative mortality rate for the comparative assessment of the quality of surgical care: results of the National Veterans Affairs Surgical Risk Study." Journal of the American College of Surgeons 185(4): 315-327.

Klabunde, C. N., et al. (2000). "Development of a comorbidity index using physician claims data." Journal of clinical epidemiology 53(12): 1258-1267.

Knaus, W. A., et al. (1991). "The APACHE III prognostic system. Risk prediction of hospital mortality for critically ill hospitalized adults." Chest Journal 100(6): 1619-1636.

Kohn, L. T., et al. (2000). To Err Is Human: Building a Safer Health System. . Washington D.C., National Accademy Press.

Krakauer, H., et al. (1992). "Evaluation of the HCFA model for the analysis of mortality following hospitalization." Health Services Research 27(3): 317.

Kristensen, S., et al. (2007). "Establishing a Set of Patient Safety Indicators." Safety Improvements for Patients in Europe Working package 4.

Lau, H. and K. C. Litman (2011). "Saving lives by studying deaths: using standardized mortality reviews to improve inpatient safety." Joint Commission Journal on Quality and Patient Safety 37(9): 400-408.

Leape, L. L. (2002). "Reporting of adverse events." The New England journal of medicine 347(20): 1633-1638.

Leape, L. L. and D. M. Berwick (2005). "Five years after To Err Is Human: what have we learned?" JAMA 293(19): 2384-2390. 
Leape, L. L., et al. (1991). "The Nature of Adverse Events in Hospitalized Patients." New England Journal of Medicine 324(6): 377-384.

Leatherman, S., et al. (1991). "Quality screening and management using claims data in a managed care setting." QRB. Quality review bulletin 17(11): 349-359.

Localio, A. R., et al. (1997). "The public release of hospital and physician mortality data in Pennsylvania: A case study." Medical Care 35(3): 272-286.

Lohr, K. N. and S. A. Schroeder (1990). "A Strategy for Quality Assurance in Medicare." New England Journal of Medicine 322(10): 707-712.

Lu, et al. (2002). A Review of Capitation in Alberta: A Report to the APP Subcommittee of Alberta Health \& Wellness and the Alberta Medical Association.

Mant, J., et al. (1997). "Epidemiology of genital prolapse: observations from the Oxford Family Planning Association Study." BJOG: An International Journal of Obstetrics \& Gynaecology 104(5): 579-585.

Martin, K. E., et al. (2004). Health-based risk assessment: risk-adjusted payments and beyond, AcademyHealth.

Mattke, S., et al. (2006). "The OECD health care quality indicators project: history and background." International Journal for Quality in Health Care 18(suppl 1): 1-4.

McClellan, M. and D. Staiger (1999). "The Quality of Health Care Providers." National Bureau of Economic Research Working Paper Series No. 7327.

McClellan, M. and D. Staiger (2000). "Comparing the Quality of Health Care Providers." Forum for Health Economics \& Policy 3.

McDonald, C. J., et al. (2000). "Deaths due to medical errors are exaggerated in Institute of Medicine report." JAMA : the journal of the American Medical Association 284(1): 93-95.

McDonald, K. M., et al. (2002). Measures of Patient Safety Based on Hospital Administrative Data - The Patient Safety Indicators. Technical Review Number 5. AHRQ Publication No. 020038. 
McKillop, I., et al. (2001). "The Financial Management of Acute Care in Canada: A Review of Funding." Performance Monitoring and Reporting Practices Canadian Institute for Health Information.

McLoughlin, V., et al. (2006). "Selecting indicators for patient safety at the health system level in OECD countries." International Journal for Quality in Health Care 18(suppl 1): 14-20.

Ministry of Health (2000). The New Zealand Health Strategy. Wellington, Ministry of Health

Ministry of Health (2003). Improving Quality (IQ): A Systems Approach for the New Zealand Health and Disability Sector., Ministry of Health.

Ministry of Health (2005). Hospital Throughput 03/04. Wellington, Ministry of Health.

Ministry of Health (2015). Mortality and Demographic Data 2012.

Murgolo, M. S. (2002). "MCBS Highlights." Health Care Financing Review 24(1): 177.

National Casemix and Classification Centre (2011). "Australian Refined Diagnosis Related Groups (AR-DRGs)."

National Casemix and Classification Centre (2011). "International Statistical Classification of Diseases and Related Health Problems, Tenth Revision, Australian Modification (ICD-10AM)."

National Health Board Business Unit (2010). National Minimum Dataset (Hospital Events) Data Dictionary. Wellington: Ministry of Health., Ministry of Health.

National Health Service (2000). An organisation with a memory: A report of an expert group on learning from adverse evens in the NHS. London, Department of Health.

National Patient Safety Agency (2008). Your NRLS. Improving the National Reporting and Learning System.

National Patient Safety Agency (2011). National Patient Safety Agency: Annual Report and Accounts 2010/11, National Patient Safety Agency. 
National Quality Forum (2006). Standardizing a Patient Safety Taxonomy: a consensus report. N. Q. Forum. Washington DC.

National Quality Forum (2008). "National Quality Forum Endorses Consensus Standards for Quality of Hospital Care.". Retrieved October 26th, 2014, from http://www.qualityforum.org/news/releases/051508-endorsed-measures.asp.

New Zealand Health Information Service (2011). "ICD-10-AM, ACHI, ACS."

Nolte, E. (2010). International benchmarking of healthcare quality: A review of the literature Santa Monica, CA, RAND Corporation.

Nuckols, T. K., et al. (2007). "Rates and types of events reported to established incident reporting systems in two US hospitals." Quality and Safety in Health Care 16(3): 164-168.

Olsen, S., et al. (2007). "Hospital staff should use more than one method to detect adverse events and potential adverse events: incident reporting, pharmacist surveillance and local real-time record review may all have a place." Quality and Safety in Health Care 16(1): 4044.

Pencina, M. J., et al. (2008). "Evaluating the added predictive ability of a new marker: from area under the ROC curve to reclassification and beyond." Statistics in Medicine 27(2): 157172.

Pope, G. C., et al. (1998). "Evaluating alternative risk adjusters for Medicare." Health Care Financing Review 20(2): 109.

Pronovost, P. J., et al. (2005). "Defining and measuring patient safety." Critical care clinics 21(1): 1-19.

Quan, H., et al. (2005). "Coding Algorithms for Defining Comorbidities in ICD-9-CM and ICD10 Administrative Data." Medical Care 43(11): 1130-1139.

Raleigh, V. S., et al. (2008). "Patient safety indicators for England from hospital administrative data: case-control analysis and comparison with US data." BMJ 337.

Reserve Bank of New Zealand (2015). "M5 Gross Domestic Product." from http://www.rbnz.govt.nz/statistics/tables/m5/. 
Riley, G., et al. (1993). "Medicare beneficiaries: adverse outcomes after hospitalization for eight procedures." Medical Care 31(10): 921-949.

Rivard P, et al. (2006). "Is There an Association Between Patient Safety Indicators and Hospital Teaching Status? ."

Rivard, P. E., et al. (2008). "Using patient safety indicators to estimate the impact of potential adverse events on outcomes." Medical Care Research and Review 65(1): 67-87.

Romano P, et al. (2002). "Can Administrative Data Be Used to Ascertain Clinically Significant Postoperative Complication Rates across Hospitals?" Medical Care 40(10): 856-867.

Roos, L. L. and R. Brazauskas (1990). "Outcomes and quality assurance: facilitating the use of administrative data." International Journal for Quality in Health Care 2(1): 77-88.

Rosen, A. K., et al. (2012). "Validating the patient safety indicators in the Veterans Health Administration: do they accurately identify true safety events?" Medical Care 50(1): 74-85.

Rosen, A. K., et al. (2009). "Effects of resident duty hour reform on surgical and procedural patient safety indicators among hospitalized VA and Medicare patients." Medical Care 47(7): 723.

Rosen, A. K., et al. (2005). "Evaluating the Patient Safety Indicators: How Well Do They Perform on Veterans Health Administration Data?" Medical Care 43(9): 873-884.

Rosen, A. K., et al. (2006). "Tracking rates of Patient Safety Indicators over time: lessons from the Veterans Administration." Medical Care: 850-861.

Runciman, W. B., et al. (2000). "A comparison of iatrogenic injury studies in Australia and the USA II: reviewer behaviour and quality of care." International Journal for Quality in Health Care 12(5): 379-388.

Salmond C, et al. (2007). NZDep Index of Deprivation users manual, University of Otago, Wellington.

Sari, A. B.-A., et al. (2007). "Sensitivity of routine system for reporting patient safety incidents in an NHS hospital: retrospective patient case note review." BMJ 334(7584): 79. 
Schone, E. and R. Brown (2013). "Risk Adjustment: What Is the Current State of the Art and How Can it Be Improved?" Princeton, NJ: Robert Wood Johnson Foundation.

Seddon, M. E., et al. (2013). "The Adverse Drug Event Collaborative: a joint venture to measure medication-related patient harm." Journal of the New Zealand Medical Association 126(1368).

Shahian, D. M., et al. (2001). "Cardiac surgery report cards: comprehensive review and statistical critique." The Annals of thoracic surgery 72(6): 2155-2168.

Sharek, P. J. (2012). "The Emergence of the Trigger Tool as the Premier Measurement Strategy for Patient Safety." AHRQ WebM\&M: morbidity \& mortality rounds on the Web 2012(5).

Shaughnessy, P. W. and D. F. Hittle (2002). "Overview of risk adjustment and outcome measures for home health agency OBQI reports: Highlights of current approaches and outline of planned Enhancements." Center for Health Services Research, University of Colorado Health Sciences Center: 1-22.

Shwartz, M., et al. (2005). "Risk adjustment and risk-adjusted provider profiles." International Journal of Healthcare Technology and Management 7(1-2): 15-42.

Statistics New Zealand (2016). "Integrated Data Infrastructure." 2016, from http://www.stats.govt.nz/browse_for_stats/snapshots-of-nz/integrated-datainfrastructure.aspx.

Stein, C. (1956). Inadmissibility of the usual estimator for the mean of a multivariate normal distribution. Proceedings of the Third Berkeley symposium on mathematical statistics and probability.

Thomas, E. J., et al. (2000). "Incidence and Types of Adverse Events and Negligent Care in Utah and Colorado." Medical Care 38(3): 261-271.

Thomas, E. J., et al. (2000). "A comparison of iatrogenic injury studies in Australia and the USA I: context, methods, casemix, population, patient and hospital characteristics." International Journal for Quality in Health Care 12(5): 371-378.

Thomas, E. J. E., et al. (1999). "Costs of medical injuries in Utah and Colorado." Inquiry : a journal of medical care organization, provision and financing 36(3): 255-264. 
Thomas, N., et al. (1994). "Empirical Bayes methods for estimating hospital-specific mortality rates." Statistics in Medicine 13(9): 889-903.

Treasury (2015). Budget Economic and Fiscal Update 2015.

Tsang, C., et al. (2008). "Patient safety indicators: A systematic review of the literature." London, UK: Dr. Foster Unit, Imperial College.

Van de Ven, W. P., et al. (2003). "Risk adjustment and risk selection on the sickness fund insurance market in five European countries." Health policy 65(1): 75-98.

Van de Ven, W. P., et al. (2004). "Health-adjusted premium subsidies in the Netherlands." Health Affairs 23(3): 45-55.

Vincent, C. (1997). "Risk, safety, and the dark side of quality." BMJ: British Medical Journal 314(7097): 1775.

Vincent, C. (2006). Patient safety, Churchill Livingstone Edinburgh.

Vincent, C., et al. (2001). "Adverse events in British hospitals: preliminary retrospective record review." BMJ 322(7285): 517-519.

West, A. N., et al. (2008). "Rare adverse medical events in VA inpatient care: reliability limits to using patient safety indicators as performance measures." Health Services Research 43(1p1): 249-266.

Wilson RM, et al. (1995). "The Quality in Australian Health Care Study." Medical Journal of Australia.November et al 163(6).

World Health Organization (2006). Promoting Patient Safety at Healthcare Institutions. New Delhi, World Health Organization.

World Health Organization (2006). Quality of Care: A Process for Making Strategic Choices in Health Systems. Geneva, World Health Organization.

World Health Organization (2006). WHO Collaborating Centre on Patient Safety (Solutions): New Release. Washington, D.C., World Health Organization. 
World Health Organization (2009). WHO Patient Safety Research. Geneva, World Health Organization.

World Health Organization (2010). "International Classification of Diseases (ICD)."

World Health Organization (2012). "Patient Safety." from http://www.who.int/patientsafety/about/en/index.html.

Wray N, et al. (1997). "Case-mix adjustment using administrative databases: a paradigm to guide future research." MCRR 54(3): 326-356.

Wu, A. W., et al. (2002). "ICU incident reporting systems." Journal of critical care 17(2): 8694.

Zhan C and Miller M (2003). "Excess Length of Stay, Charges, and Mortality Attributable to Medical Injuries During Hospitalization." JAMA 290(14): 1868-1874.

Zhan C and Miller MR (2003). "Administrative Data Based Patient Safety Research: A Critical Review. ." Qual Saf Health Care(12): 58-63. 


\section{Appendix A}

The following discusses each of the PSIs in more detail. It attempts to discuss in lay terms the adverse events the PSIs identify, how they can occur, how they can be prevented and hence why the PSIs help to quantify hospital safety and quality.

\section{PSI1 - Complications with Anaesthesia}

This indicator aims to flag events where anaesthetic overdose or reaction has occurred, or when the endotracheal tube ( $a$ tube used to facilitate breathing under anaesthesia) is misplaced. Such complications with anaesthesia are extremely rare.

An anaesthetist must induce anaesthesia and maintain patients in a safe state while they are unconscious, monitoring fluid balance, temperature, breathing, and blood loss. Complications can arise if this safe state is not maintained or if there is an adverse reaction to the medication that is given. The latter can be predictable if the patient has a history of problems with anaesthesia, or this could be an anomaly. Predictable reactions can be due to various conditions, such as poor lungs, or due to medications a patient may be taking. Maintaining the patient in a safe state should be relatively straightforward if the patient's vital signs are monitored accurately.

The endotracheal tube should be placed into the trachea to maintain an open airway for breathing, however sometimes it can be mistakenly put into the oesophagus. Tube misplacement can lead to serious comorbidity or death.

\section{PSI2 - Death in Low-Mortality DRGs}

This indicator flags in-hospital deaths which occur when the DRG (groupings of similar diagnoses) assigned to the patient is one which is considered to be low risk. The low risk DRGs are those which have a probability of dying of $0.5 \%$ or less. For PSI2 applied to New Zealand data these low risk DRGs have been identified based on nine years of NMDS, 2001- 
2009. The assumption that underpins this indicator is that if a patient who is admitted for something which is considered extremely low risk dies, it is most likely that some kind or health care error is responsible for the complication and subsequent death.

\section{PSI3 - Decubitus Ulcer}

This indicator flags cases of decubitus ulcers occurring in hospital. A decubitus ulcer, often referred to as a pressure sore or bed sore, usually develops in hospital patients when someone sits or lies in one position for too long. Prevention of pressure ulcers can take many forms but the most common is consistent rotation of the patient and keeping the skin clean and dry. Having enough sufficiently well trained nurses on a ward is important. Good nutrition can also aid the prevention of decubitus ulcers.

\section{PSI4 - Failure to Rescue}

This indicator aims to identify patients who die following a complication which develops during care. The underlying assumption is that good hospitals identify these complications quickly, treat them aggressively, and therefore avoid such deaths. The complications are specified and primarily include: pneumonia, deep vein thrombosis (DVT)/ pulmonary embolism (PE), sepsis, acute renal failure, cardiac arrest, and gastrointestinal haemorrhage/acute ulcer.

Hospitals in New Zealand are investing significantly into early warning scores and response teams to limit these complications. Most hospitals are employing hospital floor staff to regularly monitor patients and create a score based on a number of variables which are tracked over time. This enables staff to identify when a patient is deteriorating, recognise where the threshold is, know when to respond, and be able to respond quickly and effectively. Early warning teams placed in intensive care visit patients on receipt of an early warning score alarm, asses the patient in the ward and suggest intervention to prevent needing further support, or provide intensive care support directly in a more timely way. If such systems are in place and are functioning well a high number of deaths should be avoided. 


\section{PSI5 - Foreign Body Left During Procedure}

This indicator flags cases of a foreign body left accidentally in a patient during a procedure. Examples of such foreign bodies are sponges, gauze, surgical instruments, and surgical gloves.

Surgical checklists have been widely employed to avert such complications. However, errors can still occur particularly when medical staff follow the lead without doing their own count. Hierarchy can be a factor in such errors as well, such as senior staff members overriding another's concerns, or potentially when lower level staff do not voice their concerns out of fear of disagreeing or challenging a senior's position.

\section{PSI6 - latrogenic Pneumothorax}

This indicator flags cases of iatrogenic pneumothorax; a collapsed or punctured lung resulting from medical care. As a result the lung will deflate, will not function and the patient will effectively lose half their lungs. If you are old or have poor lungs already this can be life threatening.

A pneumothorax can be purposeful or accidental, however the indicator intends to identify only accidental pneumothorax. A pneumothorax can occur any time invasive work is done around the chest. The cause is often an incidental event during procedures such as inserting or removing such things as pace-makers or central lines.

\section{PSI7 - Selected Infections Due to Medical Care}

This PSI flags infections occurring during care, in particular those related to intravenous (IV) lines and catheters. IV lines are typically used for people who need fluids, blood, medications or anything given through an IV line. IV lines and catheters create a portal for infections to enter the blood stream and if not managed well infections may result. The longer they are in place the more likely an infection will occur. 
Prevention of such infections centres around how they are put in and how they are monitored. Contributors to such infections can be a lack of hand cleanliness of the person inserting the IV or catheter, lack of use of antiseptic during insertion, and irregular dressing changes.

\section{PSI8 - Postoperative Hip Fracture}

This indicator is intended to capture hip fractures that occur after surgery, most commonly a result of the patient falling. This is considered a quality issue as it generally occurs when patients are not accompanied by a staff member when walking, are mobilised inappropriately or too early after surgery, or fall out of bed.

An additional reason falling can (partly) be a fault of care as it is much more likely in patients with more complicated medical situations who should be identified in hospital and specific care management provided. Such patients can be confused if their care has not been optimised; they do not have enough fluids, correct medication, or have a lack of good nursing care around them and can fall as a result. Appropriate care should include making their environment safer with lower beds, and controlled or limited mobilisation and these things have not been put into place it could be argued there is a patient safety issue. There is however an inevitability that people can fall regardless of the care provided.

\section{PSI9 - Postoperative Haemorrhage or Hematoma}

This indicator is intended to capture events of bleeding (haemorrhaging) or build ups of blood in a post-surgical site (hematoma) that occur after any surgical procedure. The bleeding may occur immediately after the surgery or there may be a delay.

Some of the causes of bleeding may be blood clotting problems or blood vessel clamps coming undone. Both of these issues can occur as a direct result of poor surgical work but in many cases may occur regardless of surgical quality. 
Good control of bleeding vessels during surgery such as cauterising or tying off vessels that are bleeding or likely to bleed, and good monitoring after surgery (in recovery room and ward) will lower the chances of complications. If bleeding occurs it should be noticed and if significant enough, dealt with accordingly. Active monitoring of blood loss, blood pressure, pulse rate, and other attributes of the state of the patient such as dehydration potentially due to on-going bleeding are all part of creating a safe environment for the patient.

\section{PSI10 - Postoperative Physiologic and Metabolic Derangements}

This indicator flags the development of disorders that interfere with the biochemical processes within the body including kidney failure and diabetes occurring in patients after an elective surgery.

Surgery is a huge stress on the body, particularly for elderly or people with other conditions. The pancreas may not be able to cope and if the patient is not given wellmanaged fluids they can become diabetic or have other difficulties with their kidneys. Dehydration can result in kidney failure. Therefore skill is required in ensuring patients' fluids, salts, and sugar levels are being appropriately managed.

The reason the denominator for this indicator is restricted to those people who have had elective surgery is that they are considered less risky and are likely not to have so many preexisting conditions. They are also people for whom it is presumed that the benefits of surgery outweigh the risks. For these reasons the complications flagged by this indicator are considered more likely to be the result of medical error.

\section{PSI11 - Postoperative Respiratory Failure}

This indicator flags cases of postoperative respiratory failure occurring after elective surgery. Respiratory failure results in the failure of the lungs to properly complete one or both of the main tasks; taking in oxygen from the air and getting it into the bloodstream, and eliminating carbon dioxide (CO2) from the blood through air that is exhaled. This is another organ failure issue much like PSI10. Those most at risk are elderly people or those 
with substantial medical problems already. The trauma from surgery or direct trauma because a patient has had a lung or cardiac operation creates a risk of lung failure.

Prevention generally relates to limiting the stress people are put under from surgery so the organs do not fail. Surgery can potentially be conducted in a different way to minimise these stresses such as using a spinal rather than general anaesthetic, or reducing the time the surgery takes to complete. In addition careful monitoring during and post-surgery is required to ensure the early recognition of signs of complications particularly with respect to oxygen saturation.

\section{PSI12 - Postoperative Pulmonary Embolism or Deep Vein Thrombosis}

This PSI is intended to identify the occurrence of pulmonary embolism (PE) and/or deep vein thrombosis (DVT) post-surgery. Blood clots occur when blood thickens and clumps together and DVT is a blood clot that forms in a vein deep in the body. Most commonly, the DVT begins in the leg, but can also occur in veins within the abdominal cavity or in the arms. This clot is problematic because it stops circulation. If in the deep vein of leg it will be disabling and uncomfortable but if it remains in the leg it is not life threatening. A PE clogs the artery that provides blood supply to part of the lung; it is life-threatening, and is the end result of a DVT or blood clot elsewhere which has travelled up through the bloodstream to the lungs. A PE not only prevents the exchange of oxygen and carbon dioxide, but also decreases blood supply to the lung tissue itself, potentially causing lung tissue to die.

Prevention can involve mobilising patients early; surgeons electing to have spinal as opposed to general anaesthetic, or more proactive attention from medical staff to make sure a patient is being mobilised appropriately. A focus is now on providing physiotherapy and encouraging exercise statically (in bed or sitting down). Higher registered nurse hours are also generally thought to reduce the incidence of DVT and PE. Prophylactic (preventative) injections to thin the blood are often used to prevent clots, as are vascular compression stockings. 
Note: The downside of blood thinning injections is that thinning blood can result in bleeding. In addition mobilising patients inappropriately may result in falls. Therefore arguments can be made that PSI12, PSI8, and PSI9 be monitored simultaneously to prevent perverse results.

\section{PSI13 - Postoperative Sepsis}

This indicator flags cases of postoperative sepsis occurring after elective surgery. Sepsis is a condition where an infection enters the bloodstream and spreads throughout the body. Common sources of infection include surgical wounds, surgical drains, and areas of skin breakdown (e.g., bedsores), but also in areas such as the chest (pneumonia) where people who have had a tube inserted to assist with breathing while anaesthetised are at risk. Sepsis can be a life-threatening illness, particularly when it affects people with co-existing medical conditions or weakened immune systems.

There is a range of preventative measures in place to avoid postoperative sepsis. Simple things like hand washing programmes are widely employed to avoid infections, sterile dressings applied after surgery and during many procedures prophylactics are given. . Monitoring is again considered key in avoiding such complications.

Note: IVs and catheters also create risks of sepsis but these infections are captured in PSI7.

\section{PSI14 - Postoperative Wound Dehiscence}

This indicator flags cases of wound dehiscence in patients who have undergone abdominal and pelvic surgery. Wound dehiscence is the opening of the surgically closed wound which can occur as a result of poor surgical skill in closing a wound after surgery.

This indicator typically reflects surgical skill; how the wound was originally closed, whether the correct sutures were used, and of they were used with the appropriate level of skill. Amongst other things wound dehiscence can be prevented through adequate undermining (separating the skin from the underlying tissue so it can be stretched to cover the wound) 
to reduce stress on the wound edges, using sterile strips to cover the sutures for up to a week, antibiotics and cleaning the wound.

\section{PSI15 - Accidental Puncture or Laceration}

This indicator is intended to flag cases of complications that arise due to technical difficulties in medical care, specifically those involving an accidental puncture or laceration. During surgery accidental cutting of other organs or tissues in the body can occur, typically near the surgical site. This measure is important because some accidental cuts or lacerations during medical or surgical procedures can require additional surgery or treatment result in have longer-term consequences. While some patients or procedures have higher risks than others, many of these complications may be preventable.

Generally this indicator directly reflects surgical skill and therefore avoiding such complications rests with the surgeon and the level of expertise. However, indirectly these complications (as do many others) can also speak to a systems framework. If it is deemed that a complication arose due to surgeon error, questions may be asked such as: Was the correct person with the adequate experience and expertise conducting the surgery? If not, why not? Were adequate structures, processes and support in place? All of these questions suggest potentially deeper safety issues at a system level beyond those which rest with the surgeon concerned.

\section{PSI16 - Transfusion Reaction}

This indicator is intended to capture cases of major reaction due to blood transfusion. Flagged cases are only those which result in additional medical care (major reactions) as opposed to minor reactions which are less clearly due to medical error. These reactions generally involve an immune response to the blood product that was given. The most frequent signs of reactions are fever, chills, severe itching, or rashes, which typically resolve promptly without specific treatment or complications. Other signs such as severe shortness of breath, red urine, high fever, or loss of consciousness may be the first indication of a 
more severe, potentially fatal reaction. Transfusion reactions occur when the wrong blood is given to the wrong patient. These days such adverse events are relatively rare. A clerical check of the information on the blood unit label and the patient's identification should be performed to ensure that the "right" blood unit was administered to the "right" patient and an effective communication system with the laboratory established.

\section{PSI17 - Birth Trauma - Injury to Neonate}

This PSI is intended to identify cases of birth trauma for infants born in a hospital. Babies born pre-term are excluded from this indicator as traumas for these patients are considered less preventable. Neonate birth traumas are typically injuries to the new-born's head, neck, or shoulder caused during the birthing process, however, this indicator also flags injuries to the rest of the body and cases of brain damage.

Injuries to the infant usually result from mechanical forces (i.e. compression, traction) during the birth process. For example a new-born may get a haematoma to the skull caused by the head being grabbed using a ventouse (suction cup) when being delivered. Larger infants are more susceptible to birth trauma as typically more force is required. Brain damage may occur if the birth has taken too long.

Nearly one half of these complications are potentially avoidable with recognition and anticipation of obstetric risk factors. In particular the use of instruments during delivery such as forceps or vacuum.. Therefore health care standards and skilled labour can reduce the risk and likelihood of such injuries.

\section{PSI18 - Obstetric Trauma - Vaginal with Instrument}

This PSI is intended to capture cases of potentially preventable trauma to the woman giving birth when instruments (ventouse and forceps) are used. These traumas consist mainly of tears to the vagina through skin and muscles to different degrees including tears to the perineal muscles (muscles generally around the genitals and anus), anal sphincter, and 
bowel wall. Major tears will give rise to significant morbidity and may require surgical treatment after birth.

These types of tears are generally not thought to be preventable as such, but can be reduced by employing appropriate labour management and care standards. Research has shown that enhanced midwifery skills have reduced obstetric trauma rates and delays in instrument use can cause injury, as can inappropriate instrument use.

\section{PSI19 - Obstetric Trauma - Vaginal without Instrument}

This PSI is intended to capture cases of potentially preventable trauma during birth when instruments are not used. For details of the traumas flagged by this indicator see PSI18.

Giving birth without the aid of instruments is considered the "normal" birth. If a trauma occurs in this situation it is generally considered preventable and speaks to a process or recognition issue; could something have been done faster or slower in terms of how the birth was managed; should instruments have been used? As per PSI18 prevention of such traumas rest with employing appropriate labour management and care standards.

\section{PSI20 - Obstetric Trauma - Caesarean Delivery}

This PSI is intended to capture cases of potentially preventable trauma during birth by Csection. These traumas include lacerations to the perennial, cervix, bladder, rectum and/or ruptured uterus.

Generally these complications arise (and are considered preventable) when a caesarean delivery has been ordered too late by the medical staff. In these cases the traumas will typically occur before the caesarean section.

As per the previous two PSIs prevention of such traumas rest with employing appropriate labour management and care standards. 


\section{Appendix B}

Table 14: Manual mapping of NMDS AN-DRG v3.1 to DRGs used by AHRQ PSIs v3.0a

\begin{tabular}{|c|c|c|c|}
\hline $\begin{array}{l}\text { AN- } \\
\text { DRG } \\
\text { V3.1 }\end{array}$ & (NMDS) Description & $\begin{array}{l}\text { AHRQ } \\
\text { DRG }\end{array}$ & (AHRQ) Description \\
\hline 1 & Mouth larynx or pharynx disorder with tracheostomy age $>15$ & 7704 & TRACHEOSTOMY FOR FACE,MOUTH \& NECK DIAGNOSES \\
\hline 2 & Mouth larynx or pharynx disorder with tracheostomy age $<16$ & 7704 & \\
\hline 4 & $\begin{array}{l}\text { Tracheostomy except for mouth, larynx or pharynx disorder age } \\
<16\end{array}$ & 7705 & TRACH W MV 96+HRS OR PDX EXC FACE, MTH, FACE \& NECK DX W/MAJ OR \\
\hline 6 & Bone marrow transplant & 7703 & BONE MARROW TRANSPLANT \\
\hline 7 & Multiple organs transplant & 7708 & PANCREAS TRANSPLANT \\
\hline 8 & Heart transplant & 7701 & HEART TRANSPLANT \\
\hline 9 & Lung transplant & 7706 & LUNG TRANSPLANT \\
\hline 10 & ECMO without cardiac surgery & & \\
\hline 19 & $\begin{array}{l}\text { Non-acute quadriplegia or paraplegia with or without OR } \\
\text { procedure }\end{array}$ & & \\
\hline 23 & Craniotomy with CC & 101 & CRANIOTOMY AGE >17 W CC \\
\hline 24 & Craniotomy without CC & 101 & CRANIOTOMY AGE >17 WO CC \\
\hline & & & CRANIOTOMY WITH IMPLANTATION OF CHEMOTHERAPEUTIC AGENT OR ACUTE \\
\hline 25 & Spinal procedures with CC & 103 & SPINAL PROCEDURES (NO LONGER VALID) \\
\hline 26 & Spinal procedures without CC & 103 & SPINAL PROCEDURES W CC \\
\hline
\end{tabular}


27 Extracranial vascular procedure with major CC

28 Extracranial vascular procedure with non-major CC

29 Extracranial vascular procedures without CC

30 Carpal tunnel release

31 Procedure for cerebral palsy, muscular distrophy or neuropathy with CC

32 Procedure for cerebral palsy, muscular distrophy, neuropathy w/out CC

42 Viral meningitis

45 Seizure age $>64$ with $C C$

33 Peripheral and cranial nerve and other nervous system proc age $>54$

34 Peripheral and cranial nerve and other nervous system proc age $<55$

$35 \quad$ Admission for plasmapheresis

36 Plasmapheresis with neurological disease

37 Cerebrovascular disorders except TIA with CC

38 Cerebrovascular disorders except TIA without CC

$39 \quad$ Cranial and peripheral nerve disorders with CC

40 Cranial and peripheral nerve disorders without CC

41 Nervous system infection except viral meningitis

43 Prolonged monitoring for complex epilepsy

44 Nontraumatic stupor and coma

46 Seizure (age $<65$ with CC) or (age $>64$ without CC)

47 Seizure age $<65$ without CC

106

Headache

Febrile convulsions age $<5$

Severe head injury

Moderate head injury

Minor head injury
SPINAL PROCEDURES WO CC

EXTRACRANIAL VASCULAR PROCEDURES (NO LONGER VALID)

EXTRACRANIAL PROCEDURES W CC

EXTRACRANIAL PROCEDURES WO CC

CARPAL TUNNEL RELEASE

\section{PERIPH \& CRANIAL NERVE \& OTHER NERV SYST PROC W CC}

PERIPH \& CRANIAL NERVE \& OTHER NERV SYST PROC WO CC

NONSPECIFIC CEREBROVASCULAR DISORDERS W CC NONSPECIFIC CEREBROVASCULAR DISORDERS WO CC CRANIAL \& PERIPHERAL NERVE DISORDERS W CC CRANIAL \& PERIPHERAL NERVE DISORDERS WO CC NERVOUS SYSTEM INFECTION EXCEPT VIRAL MENINGITIS VIRAL MENINGITIS

NONTRAUMATIC STUPOR \& COMA SEIZURE \& HEADACHE AGE $>17$ W CC SEIZURE \& HEADACHE AGE >17 WO CC 
Other disorders of nervous system without CC

56 Dementia and global disturbances of cerebral function

57 Cerebral palsy age $>3$

58 Cerebral palsy age $<4$

59 Nervous system neoplasms age $>64$

60 Nervous system neoplasms age 25-64 108

61 Nervous system neoplasms age $<25 \quad 108$

62 Degenerative nervous system disorders age $>59 \quad 109$

63 Degenerative nervous system disorders age $<60 \quad 109$

64 Multiple sclerosis and cerebellar ataxia with CC 110

65 Multiple sclerosis and cerebellar ataxia age $>44$ without CC $\quad 110$

66 Multiple sclerosis and cerebellar ataxia age $<45$ without CC 110

67 TIA and precerebral occlusion age $>79$ with CC 127

68 TIA and precerebral occlusion (age $<80$ with CC) or (age $>79 \quad 127$ without CC)

69 TIA and precerebral occulsion age $<80$ without CC

\section{Hyphema}

84 Neurological and vascular disorders

85 Other disorders of the eye with CC

86 Other disorders of the eye without CC

88 Acute and major infections of the eye age $>54$

89 Acute and major infections of the eye age $<55$

91 Multiple eye procedures

92 Orbital procedures

93 Retinal procedures with CC

94 Retinal procedures without CC

95 Corneal, scleral and conjunctival procedures

96 Glaucoma procedures with CC

97 Glaucoma procedures without CC
OTHER DISORDERS OF NERVOUS SYSTEM W CC OTHER DISORDERS OF NERVOUS SYSTEM WO CC

NERVOUS SYSTEM NEOPLASMS W CC NERVOUS SYSTEM NEOPLASMS WO CC

DEGENERATIVE NERVOUS SYSTEM DISORDERS

MULTIPLE SCLEROSIS \& CEREBELLAR ATAXIA

TRANSIENT ISCHEMIA

HYPHEMA

OTHER DISORDERS OF THE EYE AGE $>17 \mathrm{~W}$ CC OTHER DISORDERS OF THE EYE AGE > 17 WO CC ACUTE MAJOR EYE INFECTIONS

ORBITAL PROCEDURES

RETINAL PROCEDURES 
Lens procedures with vitrectomy or with CC

99 Lens procedures without vitrectomy and without CC

Strabismus procedures

101 Eyelid procedures

102 Lacrimal procedures

103 Other eye procedures

111 Sialoadenectomy

112 Salivary gland procedures except sialoadenectomy

113 Surgical repair for cleft lip or palate diagnoses

115 Sinus, mastoid and complex middle ear procedures

117 Miscellaneous ear, nose, mouth and throat procedures

118 Rhinoplasty (with or without turbinectomy)

122 Tonsillectomy and/or adenoidectomy

124 Myringotomy with tube insertion

125 Other ear, nose, mouth and throat procedures

126 Dental and oral disorders except extractions and restorations

128 Dental extractions and restorations

130 Dysequilibrium

131 Epistaxis

132 Epiglottis

133 Otitis media and URI age $>9$ with CC

134 Otitis media and URI age $>9$ without CC

135 Otitis media and URI age $<10$

136 Laryngotracheitis

137 Nasal trauma and deformity

138 Other ear, nose, mouth and throat diagnoses with CC

139 Other ear, nose, mouth and throat diagnoses without CC

140 Ear, nose, mouth and throat malignancy-therapeutic care or major CC

141 Ear, nose, mouth and throat malignancy, other care without

\section{LENS PROCEDURES WITH OR WITHOUT VITRECTOMY}

\section{SIALOADENECTOMY}

SALIVARY GLAND PROCEDURES EXCEPT SIALOADENECTOMY

\section{CLEFT LIP \& PALATE REPAIR}

SINUS \& MASTOID PROCEDURES AGE $>17$

MISCELLANEOUS EAR, NOSE, MOUTH \& THROAT PROCEDURES

RHINOPLASTY

TONSILLECTOMY \&/OR ADENOIDECTOMY ONLY, AGE $>17$

MYRINGOTOMY W TUBE INSERTION AGE $>17$

OTHER EAR, NOSE, MOUTH \& THROAT O.R. PROCEDURES

DENTAL \& ORAL DIS EXCEPT EXTRACTIONS \& RESTORATIONS, AGE >17

DENTAL EXTRACTIONS \& RESTORATIONS

DYSEQUILIBRIUM

EPISTAXIS

EPIGLOTTITIS

OTITIS MEDIA \& URI AGE $>17$ W CC

OTITIS MEDIA \& URI AGE $>17$ WO CC

OTITIS MEDIA \& URI AGE 0-17

LARYNGOTRACHEITIS

NASAL TRAUMA \& DEFORMITY

OTHER EAR, NOSE, MOUTH \& THROAT DIAGNOSES AGE >17

EAR, NOSE, MOUTH \& THROAT MALIGNANCY 
major CC

Head and neck procedures without CC and without malignancy

146 Major head and neck procedures with CC or with malignancy

147 Other head and neck procedures with CC or with malignancy

148 Cochlear implant

149 Maxillo surgery with CC

150 Maxillo surgery without CC

151 Mouth procedures for malignant conditions

152 Mouth procedures for non-malignant conditions

160 Major chest procedures with major CC

161 Major chest procedures with non-major CC

162 Major chest procedures without CC

163 Other respiratory system OR procedures with major CC

164 Other respiratory system OR procedures with non-major CC

165 Other respiratory system OR procedures without CC

166 Respiratory system diagnosis with ventilator support

167 Pulmonary embolism age $>69$ with CC

168 Pulmonary embolism (age $>69$ without CC) or (age $<70$ with CC)

169 Pulmonary embolism age $<70$ without CC

170 Respiratory infections or inflammations age $>54$ with CC

171 Respiratory infections or inflammations (age $>54$ no $\mathrm{CC}$ )/(age $<55+$ CC)

172 Respiratory infections or inflammations age $<55$ without CC

173 Cystic fibrosis

174 Sleep apnoea with CC

175 Sleep apnoea without CC

176 Pulmonary oedema and respiratory failure

177 Chronic obstructive airways disease

178 Major chest trauma age $>69$ with CC

179 Major chest trauma (> 69 without CC) or (age $<70$ with CC)
326

326

401

401

401

402

402

402

403

403

403
MOUTH PROCEDURES W CC

MOUTH PROCEDURES WO CC

MAJOR CHEST PROCEDURES

OTHER RESP SYSTEM O.R. PROCEDURES W CC

OTHER RESP SYSTEM O.R. PROCEDURES WO CC

RESPIRATORY SYSTEM DIAGNOSIS WITH VENTILATOR SUPPORT

PULMONARY EMBOLISM

RESPIRATORY INFECTIONS \& INFLAMMATIONS AGE >17 W CC RESPIRATORY INFECTIONS \& INFLAMMATIONS AGE >17 WO CC

PULMONARY EDEMA \& RESPIRATORY FAILURE

CHRONIC OBSTRUCTIVE PULMONARY DISEASE

MAJOR CHEST TRAUMA W CC

MAJOR CHEST TRAUMA WO CC 
Pneumothorax without CC

Bronchitis and asthma age $>49$ with $\mathrm{CC}$

Bronchitis and asthma age $<50$ without CC

Whooping chough and acute bronchiolitis

Respiratory neoplasms with CC

Respiratory neoplasms without CC

and other chronic respiratory disease arising perinatally

Other respiratory problems after birth

Pleural effusion age $>64$ with $C C$

Pleural effusion (age $>64$ without CC) or (age $<65$ with CC)

Pleural effusion age $<65$ without $\mathrm{CC}$

Interstitial lung disease age $>64$ with CC

Interstitial lung disease (age $>64$ without CC) or (age $<65$ with CC)

Intistitial lung disease age $<65$ without $\mathrm{CC}$

Other respiratory system diagnoses age $>64$ with CC

Other respir syst diagnoses (age $>64$ without CC) or (age $<65$ with

CC)

201 Other respiratory system diagnoses age $<65$ without CC

221 Cardiac valve proc+pump+invasive cardiac investigative proc with CC

222 Cardiac valve proc+pump+invasive cardiac investigative proc without CC

223 Cardiac valve proc+pump without invasive cardiac proc with major CC

224 Cardiac valve proc+pump without invasive cardiac proc without major CC
RESPIRATORY SIGNS \& SYMPTOMS W CC

RESPIRATORY SIGNS \& SYMPTOMS WO CC

PNEUMOTHORAX W CC

PNEUMOTHORAX WO CC

BRONCHITIS \& ASTHMA AGE $>17 \mathrm{~W} C \mathrm{CC}$

BRONCHITIS \& ASTHMA AGE $>17$ WO CC

RESPIRATORY NEOPLASMS

PLEURAL EFFUSION W CC

PLEURAL EFFUSION WO CC

INTERSTITIAL LUNG DISEASE W CC

INTERSTITIAL LUNG DISEASE WO CC

OTHER RESPIRATORY SYSTEM DIAGNOSES W CC

OTHER RESPIRATORY SYSTEM DIAGNOSES WO CC

CARDIAC VALVE \& OTH MAJOR CARDIOTHORACIC PROC W CARD CATH

CARDIAC VALVE \& OTH MAJOR CARDIOTHORACIC PROC WO CARD CATH 
226 Other cardiothoracic procedures without pump, congenital

227 Other cardiothoracic procedures without pump, acquired

228 Major reconstructive vascular procedure without pump with major CC

229 Major reconstructive vascular procedure without pump with nonmajor CC

230 Major reconstructive vascular procedure without pump without CC

231 Vascular procedures except major reconstruction without pump with CC

232 Vascular procedures except major reconstruct without pump without CC

233 Amputation for circulatory disorders except upper limb and toe

234 Upper limb and toe amputation for circulatory disorder

236 Cardiac pacemaker implantation

239 Vein ligation and stripping

$\mathbf{2 4 0}$ Other circulatory system OR procedures

241 Implantation or replacement of AICD, total system

242 AICD component implantation or replacement

244 Circulatory system diagnosis with ventilator support

245 Circulatory disorder with AMI with invasive cardiac proc with major CC

246 Circulatory disorder with $\mathrm{AMI}+$ invasive cardiac proc without major CC

247 Circulatory disorder with AMI without invasive cardiac proc, died

248 Circulatory dis with AMI without invasive cardiac proc with major $\mathrm{CC}$

249 Circulatory dis + AMI without invasive cardiac proc without major

251 Infective endocarditis

252 Heart failure and shock

253 Venous thrombosis with major CC

507
509

510

MAJOR CARDIOVASCULAR PROCEDURES W CC

MAJOR CARDIOVASCULAR PROCEDURES WO CC

AMPUTATION FOR CIRC SYSTEM DISORDERS EXCEPT UPPER LIMB \& TOE UPPER LIMB \& TOE AMPUTATION FOR CIRC SYSTEM DISORDERS PRM CARD PACEM IMPL W AMI

PERMANENT CARDIAC PACEMAKER IMPL W MAJ CV DX OR AICD LEAD OR GNRTR VEIN LIGATION \& STRIPPING

OTHER CIRCULATORY SYSTEM O.R. PROCEDURES

CARDIAC DEFIBRILLATOR IMPLANT WO CARDIAC CATH

\section{CIRCULATORY DISORDERS W AMI \& MAIOR COMP, DISCHARGED ALIVE}

CIRCULATORY DISORDERS W AMI WO MAJOR COMP, DISCHARGED ALIVE

CIRCULATORY DISORDERS W AMI, EXPIRED

ACUTE \& SUBACUTE ENDOCARDITIS

HEART FAILURE \& SHOCK

DEEP VEIN THROMBOPHLEBITIS 

Coronary atherosclerosis with CC

256 Coronary atherosclerosis without CC

257 Hypertension with CC

258 Hypertension without CC

259 Syncope and collapse with CC

260 Syncope and collapse without CC

261 Chest pain

264 Congenital heart disease

266 Major arrhythmia and cardiac arrest with CC

267 Major arrhythmia and cardiac arrest age $>74$ without CC

268 Major arryhthmia and cardiac arrest age $<75$ without CC

269 Unstable angina with CC

270 Unstable angina without CC

271 Valvular disorders with CC

272 Valvular disorders without CC

273 Circulatory dis, no AMI+invasive cardiac proc+compl diag or + major CC

274 Circulatory dis, no AMI+invasive cardiac proc,no compl diag, without CC

275 Skin ulcers for circulatory disorders

276 Peripheral vascular disorder with major CC

277 Peripheral vascular disord (with non-major CC) or (age >74 without (C)

278 Peripheral vascular disorder age $<75$ without CC

279 Non-major arrhythmia and conduction disorders with major CC

280 Non-major arrhythmia and conduction dis age $>69$ or with nonmajor CC

281 Non-major arrhythmia and conduction disorders age $<70$ without $\mathrm{CC}$
ATHEROSCLEROSIS W CC

ATHEROSCLEROSIS WO CC

HYPERTENSION

SYNCOPE \& COLLAPSE W CC

SYNCOPE \& COLLAPSE WO CC

CHEST PAIN

CARDIAC CONGENITAL \& VALVULAR DISORDERS AGE $>17$ W CC CARDIAC CONGENITAL \& VALVULAR DISORDERS AGE $>17$ WO CC

CARDIAC ARRHYTHMIA \& CONDUCTION DISORDERS W CC CARDIAC ARRHYTHMIA \& CONDUCTION DISORDERS WO CC

\section{ANGINA PECTORIS}

\section{CIRCULATORY DISORDERS EXCEPT AMI, W CARD CATH \& COMPLEX DIAG}

CIRCULATORY DISORDERS EXCEPT AMI, W CARD CATH WO COMPLEX DIAG

PERIPHERAL VASCULAR DISORDERS W CC

PERIPHERAL VASCULAR DISORDERS WO CC 

CC)

287 Coronary bypass with invasive cardiac investigative proc with major CC

288 Coronary bypass + invasive cardiac proc age $>64$ or with nonmajor CC

289 Coronary bypass+invasive cardiac investigative proc age $<65$ without CC

290 Coronary bypass without invasive cardiac procedure with major $\mathrm{CC}$

291 Coronary bypass without invasive cardiac procedure without major CC

Other cardiothoracic or vascular procedures with pump, congenital

293 Other cardiothoracic or vascular procedures with pump, acquired

294 Cardiac pacemaker replacement with CC

295 Cardiac pacemaker replacement without CC

296 Cardiac pacemaker revision except device replacement

297 Trans-vascular percutaneous cardiac intervention

300 Stomach, oesophageal and duodenal procedures with major CC

301 Stomach, oesophageal and duodenal procedures without major

302 Stomach, oesophageal and duodenal procedures without CC

304 Pyloromyotomy procedure

305 Major small and large bowel procedures with CC

306 Major small and large bowel procedures without CC

307 Rectal resection age $>69$ with CC

308 Rectal resection (age $<70$ with CC) or (age $>69$ without CC)

309 Rectal resection age $<70$ without CC
OTHER CIRCULATORY SYSTEM DIAGNOSES W CC

OTHER CIRCULATORY SYSTEM DIAGNOSES WO CC

CORONARY BYPASS W CARDIAC CATH (NO LONGER VALID)

CORONARY BYPASS W CARDIAC CATH W MAJOR CV DX

CORONARY BYPASS W CARDIAC CATH W

CORONARY BYPASS WO PTCA OR CARDIAC CATH (NO LONGER VALID)

CORONARY BYPASS W/O CARDIAC CATH W MAJOR CV DX

CORONARY BYPASS W/O CARDIAC CATH W/O MAJOR CV DX

OTHER CARDIOTHORACIC PROCEDURES

\section{CARDIAC PACEMAKER DEVICE REPLACEMEN}

CARDIAC PACEMAKER REVISION EXCEPT DEVICE REPLACEMENT PERCUTANEOUS CARDIOVASCULAR PROCEDURES (NO LONGER VALID) STOMACH, ESOPHAGEAL \& DUODENAL PROCEDURES AGE $>17 \mathrm{~W}$ CC STOMACH, ESOPHAGEAL \& DUODENAL PROCEDURES AGE >17 WO CC

MAJOR SMALL \& LARGE BOWEL PROCEDURES W CC MAJOR SMALL \& LARGE BOWEL PROCEDURES WO CC RECTAL RESECTION W CC

RECTAL RESECTION WO CC 
310 Peritoneal adhesiolytis age $>49$ with CC

311 Peritoneal adhesiolytis (age $<50$ with CC) or (age $>49$ without CC)

312 Peritoneal adhesiolytis age $<50$ without CC

313 Appendicectomy with complicated principal diagnosis

314 Appendectomy without complicated principal diagnosis

315 Minor small and large bowel procedures with CC

316 Minor small and large bowel procedures without CC

317 Anal and stomal procedures with CC

318 Anal and stomal procedures without CC

319 Abdominal, umbilical and other hernia procedures age $>9$

320 Inguinal and femoral hernia procedures age $>9$

321 Hernia procedures age $<10$

322 Other digestive system OR procedures with $\mathrm{CC}$ or with malignancy

323 Other digestive system OR procedures without CC without malignancy

325 Complex therapeutic gastroscopy for major digestive disease with $\mathrm{CC}$

326 Complex therapeutic gastroscopy for major digestive disease without CC

327 Complex therapeutic gastroscopy for non-major digestive dis with CC

328 Complex therapeutic gastroscopy for non-major digestive dis without CC

329 Other gastroscopy for major digestive disease with CC

330 Other gastroscopy for major digestive disease without CC

331 Other gastroscopy for non-major digestive disease with CC

332 Other gastroscopy for non-major digestive disease without CC
PERITONEAL ADHESIOLYSIS W CC PERITONEAL ADHESIOLYSIS WO CC

APPENDECTOMY W COMPLICATED PRINCIPAL DIAG W CC APPENDECTOMY W COMPLICATED PRINCIPAL DIAG WO CC APPENDECTOMY WO COMPLICATED PRINCIPAL DIAG W CC APPENDECTOMY WO COMPLICATED PRINCIPAL DIAG WO CC MINOR SMALL \& LARGE BOWEL PROCEDURES W CC MINOR SMALL \& LARGE BOWEL PROCEDURES WO CC ANAL \& STOMAL PROCEDURES W CC ANAL \& STOMAL PROCEDURES WO CC HERNIA PROCEDURES EXCEPT INGUINAL \& FEMORAL AGE >17 W CC HERNIA PROCEDURES EXCEPT INGUINAL \& FEMORAL AGE >17 WO CC INGUINAL \& FEMORAL HERNIA PROCEDURES AGE >17 W CC INGUINAL \& FEMORAL HERNIA PROCEDURES AGE >17 WO CC HERNIA PROCEDURES AGE 0-17 OTHER DIGESTIVE SYSTEM O.R. PROCEDURES W CC 
335 Other colonoscopy without CC

336 Digestive malignancy

GI haemorrhage age $>64$ or with CC GI haemorrhage age $<65$ without CC

339 Complicated peptic ulcer with CC

340 Complicated peptic ulcer without CC

341 Uncomplicated peptic ulcer

342 Inflammatory bowel disease with CC

343 Inflammatory bowel disease without CC

344 Gl obstruction with CC

345 Gl obstruction without CC

346 Abdominal pain or mesenteric adenitis with CC

347 Abdominal pain or mesenteric adenitis with $\mathrm{CC}$

348 Oesophagitis,gastroenteritis, misc digest dis age $>74$ or age 10-74 $+\mathrm{CC}$

349

Oesophagitis, gastroenteritis, misc digestive dis age 10-74 without CC

350 Gastroenteritis age $<10$

351 Oesophagitis and miscellaneous digestive system disorders age $<10$

352 Other digestive system diagnoses age $>9$ with $\mathrm{CC}$

353 Other digestive system diagnoses age $>9$ without CC

354 Other digestive system diagnoses age $<10$

359 Pancreas, liver and shunt procedures with major CC

360 Pancreas, liver and shunt procedures with non-major CC

361 Pancreas, liver and shunt procedures without CC

616

618
DIGESTIVE MALIGNANCY W CC

DIGESTIVE MALIGNANCY WO CC

615 G.I. HEMORRHAGE W CC

615 G.I. HEMORRHAGE WO CC

616 COMPLICATED PEPTIC ULCER

UNCOMPLICATED PEPTIC ULCER W CC

UNCOMPLICATED PEPTIC ULCER WO CC

G.I. OBSTRUCTION W CC

G.I. OBSTRUCTION WO CC

ESOPHAGITIS, GASTROENT \& MISC DIGEST DISORDERS AGE >17 WO CC

ESOPHAGITIS, GASTROENT \& MISC DIGEST DISORDERS AGE 0-17
OTHER DIGESTIVE SYSTEM DIAGNOSES AGE $>17 \mathrm{~W}$ CC OTHER DIGESTIVE SYSTEM DIAGNOSES AGE > 17 WO CC OTHER DIGESTIVE SYSTEM DIAGNOSES AGE 0-17 PANCREAS, LIVER \& SHUNT PROCEDURES W CC PANCREAS, LIVER \& SHUNT PROCEDURES WO CC 

$\mathrm{CDE}+$ maj CC

363 Biliary tract proc except cholecyst only with/without CDE + nonmaj CC

364 Biliary tract proc except cholecyst only with/without CDE without

367 Cholecystectomy without CC

Hepatobiliary diagnostic procedures for malignancy

369 Hepatobiliary diagnostic procs for non malignancy

371 Cirrhosis and alcoholic hepatitis with CC

372 Cirrhosis and alcoholic hepatitis without CC

376 Liver disease except malignancy,cirrhosis, alcoholic hepatitis with CC

377 Liver dis except malignancy, cirrhosis, alcoholic hepatitis without CC

378 Disorders of the biliary tract with $\mathrm{CC}$

379 Disorders of the biliary tract without CC

380 Other hepatobiliary and pancreas OR procedures with CC

381 Other hepatobilliary and pancreas OR procedures with CC

382 Malignancy of hepatobiliary system or pancreas age $>69$ with CC

383 Hepatobiliary system or pancreas malignancy $(<70+C \mathrm{C}) /(>69$ without CC)

384 Malignancy of hepatobiliary system or pancreas age $<70$ without CC

385 ERCP complex therapeutic procedures for malignancy or with CC

386 ERCP complex therapeutic procedures not for malignancy without $\mathrm{CC}$

387 ERCP other therapeutic procedures for malignancy or with CC

388 ERCP other therapeutic procedures not for malignancy without $\mathrm{CC}$

CHOLECYSTECTOMY W C.D.E. W CC

CHOLECYSTECTOMY W C.D.E. WO CC

CHOLECYSTECTOMY EXCEPT BY LAPAROSCOPE WO C.D.E. W CC CHOLECYSTECTOMY EXCEPT BY LAPAROSCOPE WO C.D.E. WO CC HEPATOBILIARY DIAGNOSTIC PROCEDURE FOR MALIGNANCY HEPATOBILIARY DIAGNOSTIC PROCEDURE FOR NON-MALIGNANCY CIRRHOSIS \& ALCOHOLIC HEPATITIS

\section{DISORDERS OF LIVER EXCEPT MALIG,CIRR,ALC HEPA W CC}

DISORDERS OF LIVER EXCEPT MALIG,CIRR,ALC HEPA WO CC

DISORDERS OF THE BILIARY TRACT W CC

DISORDERS OF THE BILIARY TRACT WO CC

OTHER HEPATOBILIARY OR PANCREAS O.R. PROCEDURES

MALIGNANCY OF HEPATOBILIARY SYSTEM OR PANCREAS 

Dis pancreas except malignancy (age $<55$ with $\mathrm{CC}$ )/(age $>54$ without CC)

Bone joint infect, inflamm with misc musculoskel, connect tissue proc

401 Bilateral or multiple major joint procedures of lower limb

402 Skin graft excluding hand with $\mathrm{CC}$

403 Skin graft excluding hand without CC

404 Hip replacement with CC

405 Hip replacement without CC

406 Other major joint and limb reattachment procedures with CC

407 Other major joint and limb reattachment procedures without CC

408 Hip and femur procedures except major joint with CC

409 Hip and femur procedures except major joint age $>54$ without CC

410 Hip and femur procedures except major joint age $<55$ without CC

411 Amputation

412 Stump revision

413 Spinal fusion with scoliosis

414 Back and neck procedures or spinal fusion with malignancy or with $\mathrm{CC}$

415 Spinal fusion

416 Back and neck procedures

418 Lower extremity and humerus procs except hip, foot and femur with CC

419 Leg and humerus procs except hip, foot and femur age $>59$ without CC

420 Leg and humerus procs except hip, foot and femur age $<60$

\section{DISORDERS OF PANCREAS EXCEPT MALIGNANCY}

BILATERAL OR MULTIPLE MAJOR JOINT PROCS OF LOWER EXTREMITY WND DEBRID \& SKN GRFT EXCEPT HAND,FOR MUSCSKELET \& CONN TISS DIS

MAJOR JOINT \& LIMB REATTACHMENT PROCEDURES OF LOWER EXTREMITY (NO LONGER VALID)

MAJOR JOINT REPLACEMENT OR REATTACHMENT OF LOWER EXTREMITY REVISION OF HIP OR KNEE REPLACEMENT

HIP \& FEMUR PROCEDURES EXCEPT MAJOR JOINT AGE $>17 \mathrm{~W}$ CC HIP \& FEMUR PROCEDURES EXCEPT MAJOR JOINT AGE >17 WO CC HIP \& FEMUR PROCEDURES EXCEPT MAJOR JOINT AGE 0-17 AMPUTATION FOR MUSCULOSKELETAL SYSTEM \& CONN TISSUE DISORDERS

SPINAL FUSION EXCEPT CERVICAL W CC

BACK \& NECK PROC W CC (NO LONGER VALID)

BACK \& NECK PROC WO CC (NO LONGER VALID)

SPINAL FUSION EXCEPT CERVICAL WO CC

BACK \& NECK PROCEDURES EXCEPT SPINAL FUSION W CC

BACK \& NECK PROCEDURES EXCEPT SPINAL FUSION WO CC

LOWER EXTREM \& HUMER PROC EXCEPT HIP,FOOT,FEMUR AGE >17 W CC

LOWER EXTREM \& HUMER PROC EXCEPT HIP,FOOT,FEMUR AGE >17 WO CC

LOWER EXTREM \& HUMER PROC EXCEPT HIP,FOOT,FEMUR AGE 0-17 
without CC

421 Knee procedures

422 Soft tissue procedures

423 Local excision and removal of internal fixation device of hip or femur

424 Local excision, removal internal fixation device except hip and femur

425 Major shoulder or elbow procedures age $>59$

426 Major shoulder or elbow procedures age $<60$

427 Major thumb or joint procedures

428 Foot procedures

429 Shoulder, elbow, forearm proc except major joint age $>69$ or with CC

430 Shoulder, elbow, forearm proc except major joint age $<70$ without CC

431 Arthroscopy

432 Hand or wrist procedures except major joint

433 Maxillo-facial surgery

434 Cranio-facial surgery

435 Biopsies of musculoskeletal system and connective tissue

436 Other musculoskeletal system and connective tissue procedures with CC

437 Other musculoskeletal system and connective tissue procs without CC

438 Major fractures of femur

439 Non-major fractures of femur

440 Fractures of hip and pelvis with CC

441 Fractures of hip and pelvis age $>74$ without CC

442 Fractures of hip and pelvis age $<75$ without CC

443 Sprains, strains and dislocations of hip, pelvis and thigh
KNEE PROC W CC (NO LONGER VALID)

KNEE PROC WO CC (NO LONGER VALID)

SOFT TISSUE PROCEDURES W CC

SOFT TISSUE PROCEDURES WO CC

LOCAL EXCISION \& REMOVAL OF INT FIX DEVICES OF HIP \& FEMUR

LOCAL EXCISION \& REMOVAL OF INT FIX DEVICES EXCEPT HIP \& FEMUR (NO LONGER VALID)

MAJOR SHOULDER

SHOULDER,ELBOW OR FOREARM PROC,EXC MAJOR JOINT PROC, WO CC MAJOR THUMB OR JOINT PROC,OR OTH HAND OR WRIST PROC W CC

FOOT PROCEDURES

\section{ARTHROSCOPY}

HAND OR WRIST PROC, EXCEPT MAJOR JOINT PROC, WO CC

BIOPSIES OF MUSCULOSKELETAL SYSTEM \& CONNECTIVE TISSUE

OTHER MUSCULOSKELET SYS \& CONN TISS O.R. PROC W CC

OTHER MUSCULOSKELET SYS \& CONN TISS O.R. PROC WO CC

FRACTURES OF FEMUR

SPRAINS, STRAINS, \& DISLOCATIONS OF HIP, PELVIS \& THIGH 
446 Path \# and musculoskeletal system or conn tissue malignancy age $>64$

447 Path \# and musculoskeletal system or conn tissue malignancy age $<65$

448 Connective tissue disorders age $>64$ or with CC

449 Connective tissue disorders age $<65$ without CC

450 Septic arthritis with CC

451 Septic arthritis age $>54$ without CC

452 Septic arthritis age $<55$ without CC

453 Medical back problems age $>74$ with $\mathrm{CC}$

454 Medical back problems (age $>74$ without CC) or (age $<75$ with CC)

455 Medical back problems age $<75$ without CC

456 Bone diseases and specific arthropathies age $>74$ with CC

457 Bone diseases and specific arthropathies age $>74$ without CC

458 Bone diseases and specific arthropathies age 65-74

459 Bone diseases and specific arthropathies age $<65$

460 Non-specific arthropathies age $>69$

461 Non-specific arthropathies age $<70$

462 S\&S musculoskeletal syst and connective tissue age $>69$ with CC

$463 \mathrm{~S} \& S$ musculoskeletal syst and conn tissue (age $>69$ no CC)/(age $<70+$ CC)

464 S\&S musculoskeletal system and connective tissue age $<70$ without CC

465 Tendonitis, myositis and bursitis age $>79$

466 Tendonitis, myositis and bursitis age $<80$ with CC

467 Tendonitis, myositis and bursitis age $>80$ without CC

468 Aftercare musculoskeletal system and connective tissue age $>59$ with CC

469 Aftercare musculoskel syst and conn tissue (age $>59$ no CC) $/($ age $<60+C C)$

\section{OSTEOMYELITIS}

PATHOLOGICAL FRACTURES \& MUSCULOSKELETAL \& CONN TISS MALIGNANCY

CONNECTIVE TISSUE DISORDERS W CC

CONNECTIVE TISSUE DISORDERS WO CC

SEPTIC ARTHRITIS

MEDICAL BACK PROBLEMS

BONE DISEASES \& SPECIFIC ARTHROPATHIES W CC

BONE DISEASES \& SPECIFIC ARTHROPATHIES WO CC

NON-SPECIFIC ARTHROPATHIES

SIGNS \& SYMPTOMS OF MUSCULOSKELETAL SYSTEM \& CONN TISSUE

TENDONITIS, MYOSITIS \& BURSITIS

AFTERCARE, MUSCULOSKELETAL SYSTEM \& CONNECTIVE TISSUE 
471 Fracture,sprain,strain, dislocation forearm, hand, foot age $>74$ with 832 CC

472 Fracture,sprain, strain, dislocate forearm, hand, foot $>74$ no CC) $(<75+$ CC $)$

473 Fracture,sprain,strain, dislocation forearm, hand, foot age $>75$ no CC

474 Fracture,sprain, strain, dislocation upper arm, lower leg age $>64+$ CC

475 Fracture,sprain,strain, disloc upper arm,lower leg (>64 no CC) $(<65+$ CC)

476 Fracture,srpain,strain, dislocation upper arm, lower leg age <65 no CC

477 Other musculoskeletal system, connective tissue diagnosis $>69+C$

478 Oth musculoskeletal syst connective tissue diag(age $>69$ no $\mathrm{CC}) /(<70+\mathrm{CC})$

479 Other musculoskelal system, connective tissue diagnosis age $<70$ no CC

482 Perianal and pilonidal procedures

483 Skin and subcutaneous tissue and breast plastic procedures

484 Other skin and subcutaneous tissue and breast procedures

$488 \quad$ Non-malignant breast disorders

489 Cellulitis age $>59+\mathrm{CC}$

490 Cellulitis (age $>59$, no CC) or (age $<60+$ CC)

491 Cellulitis age $<60$, no CC

492 Trauma to skin and subcutaneous tissue of breast age $>69+C C$

493 Trauma to skin and subcutaneous tissue of breast age $>69$ no CC

494 Trauma to skin and subcutaneous tissue of breast age $<70$

495 Major procedures for malignant breast conditions

496 Minor procedures for malignant breast conditions

497 Major procedures for non-malignant breast conditions
FX, SPRN, STRN \& DISL OF FOREARM, HAND, FOOT AGE >17 W CC

FX, SPRN, STRN \& DISL OF FOREARM, HAND, FOOT AGE >17 WO CC

FX, SPRN, STRN \& DISL OF UPARM,LOWLEG EX FOOT AGE >17 W CC

FX, SPRN, STRN \& DISL OF UPARM,LOWLEG EX FOOT AGE >17 WO CC

OTHER MUSCULOSKELETAL SYSTEM \& CONNECTIVE TISSUE DIAGNOSES

PERIANAL \& PILONIDAL PROCEDURES

SKIN, SUBCUTANEOUS TISSUE \& BREAST PLASTIC PROCEDURES

OTHER SKIN, SUBCUT TISS \& BREAST PROC W CC

OTHER SKIN, SUBCUT TISS \& BREAST PROC WO CC

NON-MALIGANT BREAST DISORDERS

CELLULITIS AGE $>17 \mathrm{~W}$ CC

CELLULITIS AGE $>17$ WO CC

TRAUMA TO THE SKIN, SUBCUT TISS \& BREAST AGE >17 W CC

TRAUMA TO THE SKIN, SUBCUT TISS \& BREAST AGE $>17$ WO CC

TOTAL MASTECTOMY FOR MALIGNANCY W CC

SUBTOTAL MASTECTOMY FOR MALIGNANCY W CC

TOTAL MASTECTOMY FOR MALIGNANCY WO CC 
498 Minor procedures for non-malignant breast conditions

500 Lower limb+skin graft/flap repair+ulcer/cellulitis + CC

501 Lower limb+skin graft/flap repair+ulcer/cellulitis no CC

502 Lower limb + other OR procedure + ulcer or cellulitis

503 Lower limb+skin graft/flap repair, no ulcer or cellulitis

504 Lower limb + other OR procedure, no ulcer or cellulitis

505 Other skin graft and/or debridement procedures

506 Skin ulcers age $>64$

507 Skin ulcers age $<65$

508 Major skin disorders age $>44+\mathrm{CC}$

509 Major skin disorders age 10-44 or (> 44 no CC)

510 Major skin disorders age $<10$

511 Malignant breast disorders age $>69+\mathrm{CC}$

512 Malignant breast disorders age $(>69$ no $C C)$ or $(<70+C C)$

513 Malignant breast disorders age $<70$ no CC

514 Miscellaneous skin disorders

515 Minor skin disorders

520 Diabetic foot

521 Adrenal procedures

522 Pituitary procedures

524 Obesity procedures

525 Parathyroid procedures

526 Thyroid procedures

$\mathbf{5 2 7}$ Thyroglossal procedures

528 Other endocrine, nutritional \& metabolic OR procs

529 Same day admission for endoscopic OR procedure

531 Severe nutritional disturbance
SUBTOTAL MASTECTOMY FOR MALIGNANCY WO CC SKIN GRAFT \&/OR DEBRID FOR SKN ULCER OR CELLULITIS W CC SKIN GRAFT \&/OR DEBRID FOR SKN ULCER OR CELLULITIS WO CC

SKIN GRAFT \&/OR DEBRID EXCEPT FOR SKIN ULCER OR CELLULITIS W CC SKIN GRAFT \&/OR DEBRID EXCEPT FOR SKIN ULCER OR CELLULITIS WO CC

SKIN ULCERS

MAJOR SKIN DISORDERS W CC

MAJOR SKIN DISORDERS WO CC

MALIGNANT BREAST DISORDERS W CC

MALIGNANT BREAST DISORDERS WO CC

MINOR SKIN DISORDERS W CC

MINOR SKIN DISORDERS WO CC

ADRENAL \& PITUITARY PROCEDURES

O.R. PROCEDURES FOR OBESITY

PARATHYROID PROCEDURES

THYROID PROCEDURES

THYROGLOSSAL PROCEDURES

OTHER ENDOCRINE, NUTRIT \& METAB O.R. PROC W CC

OTHER ENDOCRINE, NUTRIT \& METAB O.R. PROC WO CC 
Endocrine disorders age $>69$

536 Endocrine disorders age $<70+\mathrm{CC}$

Endocrine disorders age $<70$ no CC

538 Same day investigation etc of metabolic disorders

539 Diabetes+major CC or (age > $59+$ non-major CC)

540 Diabetes age $>59$ no CC

541 Diabetes age $<60$ no major CC

550 Kidney transplant + CC

551 Kidney transplant no CC

552 Kidney, ureter \& major bladder procedure for neoplasm + CC

553 Kidney, ureter \& major bladder procedure for neoplasm no CC

554 Kidney, ureter \& major bladder procedure for non-neoplasm

556 Minor bladder procedures + CC

557 Minor bladder procedures, no CC

558 Prostatectomy + CC

559 Prostatectomy, no CC

$\mathbf{5 6 0}$ Transurethral procedures + major CC

561 Transurethral procedures + non-major CC

562 Transurethral procedures, no CC

563 Urethral procedures age $>9+C C$

564 Urethral procedures age $>9$, no CC

565 Urethral procedures age $<10$

566 Insertion of peritoneal catheter

567 Other kidney \& urinary tract OR procedures + major CC

568 Other kidney \& urinary tract OR procedures + non-major CC
1014

1014

1009

NUTRITIONAL \& MISC METABOLIC DISORDERS AGE >17 W CC NUTRITIONAL \& MISC METABOLIC DISORDERS AGE >17 WO CC INBORN ERRORS OF METABOLISM

ENDOCRINE DISORDERS W CC

ENDOCRINE DISORDERS WO CC

DIABETES AGE >35

DIABETES AGE 0-35

KIDNEY TRANSPLANT

KIDNEY,URETER \& MAJOR BLADDER PROCEDURES FOR NEOPLASM

KIDNEY,URETER \& MAJOR BLADDER PROC FOR NON-NEOPL W CC KIDNEY,URETER \& MAJOR BLADDER PROC FOR NON-NEOPL WO CC MINOR BLADDER PROCEDURES W CC

1105

MINOR BLADDER PROCEDURES WO CC

PROSTATECTOMY W CC

PROSTATECTOMY WO CC

TRANSURETHRAL PROCEDURES W CC

TRANSURETHRAL PROCEDURES WO CC

URETHRAL PROCEDURES, AGE $>17$ W CC

URETHRAL PROCEDURES, AGE $>17$ WO CC

URETHRAL PROCEDURES, AGE 0-17

1109

OTHER KIDNEY \& URINARY TRACT O.R. PROCEDURES 
Other kidney and urinary tract OR procedures, no CC

Renal failure + CC

Renal failure, no CC

Admit for renal dialysis

Kidney and urinary tract neoplasms + CC

Kidney and urinary tract neoplasms, no CC

Kidney and urinary tract infections age $>69+\mathrm{CC}$

Kidney and urinary tract infection age $(<70+\mathrm{CC})$ or $(>69$ no CC)

Kidney and urinary tract infections age $<70$, no CC

Urinary stones + ESW lithotripsy

Urinary stones, no ESW lithotripsy

Kidney and urinary tract Signs \& Symptoms age $>74+$ CC $+\mathrm{CC}$

Kidney and urinary tract Signs \& Symptoms age $<75$, no CC

Urethral stricture + CC

Urethral stricture, no CC

Other kidney and urinary tract diagnoses + major CC

Other kidney and urinary tract diagnoses + non-major CC

Other kidney and urinary tract diagnoses, no CC

Major male pelvic procedures + CC

Major male pelvic procedures, no CC

Penis procedures $+\mathrm{CC}$

Penis procedures, no CC

Transurethral prostatectomy age $>79+\mathrm{CC}$

Transurethral prostatectomy age $(80+C C)$ or $(>79$, no CC

Transurethral prostatectomy age $<80$, no CC

Testes procedures for malignancy + CC

Testes procedures for malignancy, no CC

Testes procedures except malignancy age $>9+\mathrm{CC}$
1109

1110

1110

1111

1112

1112

1113

1113

1113

1115

1115

1116

1116

1116

1118

1118

1120

1120

1120

1201

1201

1206

1206

1202

1202

1202

1203

1203

1204
RENAL FAILURE

ADMIT FOR RENAL DIALYSIS

KIDNEY \& URINARY TRACT NEOPLASMS W CC

KIDNEY \& URINARY TRACT NEOPLASMS WO CC

KIDNEY \& URINARY TRACT INFECTIONS AGE > $17 \mathrm{~W} C \mathrm{CC}$

KIDNEY \& URINARY TRACT INFECTIONS AGE >17 WO CC

URINARY STONES W CC, \&/OR ESW LITHOTRIPSY

URINARY STONES WO CC

KIDNEY \& URINARY TRACT SIGNS \& SYMPTOMS AGE >17 W CC

KIDNEY \& URINARY TRACT SIGNS \& SYMPTOMS AGE >17 WO CC

URETHRAL STRICTURE AGE >17 W CC

URETHRAL STRICTURE AGE $>17$ WO CC

OTHER KIDNEY \& URINARY TRACT DIAGNOSES AGE $>17$ W CC

OTHER KIDNEY \& URINARY TRACT DIAGNOSES AGE > 17 WO CC

MAJOR MALE PELVIC PROCEDURES W CC

MAJOR MALE PELVIC PROCEDURES WO CC

PENIS PROCEDURES

TRANSURETHRAL PROSTATECTOMY W CC

TRANSURETHRAL PROSTATECTOMY WO CC

TESTES PROCEDURES, FOR MALIGNANCY

TESTES PROCEDURES, NON-MALIGNANCY AGE >17 

Testes procedures except for malignancy age $<10$

612 Circumcision age $>9$

613 Circumcision age $<10$

614 Other male reproductive system OR procedures for malignancy

615 Other male reproductive system OR procedures,except malignancy

616 Malignancy of male reproductive system + CC

617 Malignancy of male reproductive system, no CC

618 Benign prostatic hypertrophy + CC

619 Benign prostatic hypertrophy, no CC

620 Inflammation of male reproductive system + CC

621 Inflammation of male reproductive system no CC

622 Sterilisation, male

Other male reproductive system diagnoses

Pelvic evisceration and radical vulvectomy

651

Uterine/adnexa procedure for non-ovarian/adnexal malignancy,

no CC

655

Uterine/adnexal procedure for non-malignancy age $>39+\mathrm{CC}$

656 Uterine/adnexal procedure for non-malignancy age( $>39$ no $\mathrm{CC})$ or $(<40+\mathrm{CC})$

657 Uterine/adnexa procedure for non-malignancy age $<40$ no CC

658 Female reproductive system reconstructive procedures

659 Conisation, vagina, cervix and vulva procedures

660 Endoscopic procedures on female reproductive system

661 Diagnostic curettage and/or diagnostic hysteroscopy

662 Oth female reproductive system OR procs age $>64$ or +
1204
TESTES PROCEDURES, NON-MALIGNANCY AGE 0-17

CIRCUMCISION AGE $>17$

CIRCUMCISION AGE 0-17

OTHER MALE REPRODUCTIVE SYSTEM O.R. PROCEDURES FOR MALIGNANCY OTHER MALE REPRODUCTIVE SYSTEM O.R. PROC EXCEPT FOR MALIGNANCY

MALIGNANCY, MALE REPRODUCTIVE SYSTEM, W CC MALIGNANCY, MALE REPRODUCTIVE SYSTEM, WO CC BENIGN PROSTATIC HYPERTROPHY W CC BENIGN PROSTATIC HYPERTROPHY WO CC INFLAMMATION OF THE MALE REPRODUCTIVE SYSTEM

STERILIZATION, MALE

OTHER MALE REPRODUCTIVE SYSTEM DIAGNOSES

PELVIC EVISCERATION, RADICAL HYSTERECTOMY \& RADICAL VULVECTOMY UTERINE \& ADNEXA PROC FOR OVARIAN OR ADNEXAL MALIGNANCY

UTERINE,ADNEXA PROC FOR NON-OVARIAN

UTERINE,ADNEXA PROC FOR NON-OVARIAN

UTERINE \& ADNEXA PROC FOR NON-MALIGNANCY W CC UTERINE \& ADNEXA PROC FOR NON-MALIGNANCY WO CC

FEMALE REPRODUCTIVE SYSTEM RECONSTRUCTIVE PROCEDURES

VAGINA, CERVIX \& VULVA PROCEDURES

ENDOSCOPIC TUBAL INTERRUPTION

OTHER FEMALE REPRODUCTIVE SYSTEM O.R. PROCEDURES 
malignancy or+CC

Oth female reproductive system OR procs age $<65$, no malignancy, no CC

Malignancy of female reproductive system age $>69$

665 Malignancy of female reproductive system age $<70$

666 Infections of the female reproductive system

667 Menstrual, other female reproductive system disorders age $>69$ or + CC

668 Menstrual, other female reproductive system disorders age $<70$, no CC

670 Caesarean delivery, no complicating diagnosis

671 Caesarean delivery + moderate complicating diagnosis

672 Caesaeran delivery + severe complicating diagnosis

674 Vaginal delivery no complicating diagnosis

675 Vaginal delivery + moderate complicating diagnosis

676 Vaginal delivery + severe complicating diagnosis

677 Vaginal delivery + complicating OR procedures

678 Postpartum, post abortion diagnoses, no OR procedure

679 Postpartum, post abortion diagnoses + OR procedure

680 Ectopic pregnancy

681 Threatened abortion

682 Abortion, no D \& C

683 Abortion + D \& C, aspiration curettage or hysterotomy

684 Preterm labour

685 Other antenatal admission + severe complicating diagnoses

686 Other antenatal admission + moderate/no complicating diagnoses

687 Caesarean delivery + mult complicating diagnoses, at least one severe

688 Vaginal delivery + mult complicating diagnoses, at least one severe

701 Neonate, died, trans $<5$ days, no significant OR proc, born here
1311
1312

1312

1313

1314

1314

1401

1401

1401

1402

1402

1402

1404

1405

1406

1407

1408

1409

1410

1411

1412

1413
MALIGNANCY, FEMALE REPRODUCTIVE SYSTEM W CC MALIGNANCY, FEMALE REPRODUCTIVE SYSTEM WO CC INFECTIONS, FEMALE REPRODUCTIVE SYSTEM

MENSTRUAL \& OTHER FEMALE REPRODUCTIVE SYSTEM DISORDERS

\section{CESAREAN SECTION W CC}

CESAREAN SECTION WO CC

VAGINAL DELIVERY W COMPLICATING DIAGNOSES

VAGINAL DELIVERY WO COMPLICATING DIAGNOSES

VAGINAL DELIVERY W O.R. PROC EXCEPT STERIL \&/OR D\&C POSTPARTUM \& POST ABORTION DIAGNOSES WO O.R. PROCEDURE POSTPARTUM \& POST ABORTION DIAGNOSES W O.R. PROCEDURE ECTOPIC PREGNANCY

THREATENED ABORTION

ABORTION WO D\&C

ABORTION W D\&C, ASPIRATION CURETTAGE OR HYSTEROTOMY

FALSE LABOR

OTHER ANTEPARTUM DIAGNOSES W MEDICAL COMPLICATIONS

OTHER ANTEPARTUM DIAGNOSES WO MEDICAL COMPLICATIONS

1502

EXTREME IMMATURITY OR RESPIRATORY DISTRESS SYNDROME, NEONATE 
702 Neonate, died, trans $<5$ days of adm + significant OR proc

1502

703 Neonate, died, trans $<5$ days, no significant OR proc, not born here

704 Cardiothoracic or vascular procedures for neonates

705 Neonate, admission weight $<750$ grams

706 Neonate, admission weight 750 - 999 grams

707 Neonate, admission weight 1000-1499g + significant OR proc

708 Neonate, admission weight 1000-1249g, no significant OR proc

709 Neonate, admission weight 1250-1499g, no significant OR proc

710 Neonate, admission weight 1500-1999g, + significant OR proc

711 Neonate, adm weight 1500-1999g, no significant OR proc + mult maj prob

712 Neonate, adm weight 1500-1999g, no significant OR proc + maj problem

713 Neonate, adm weight 1500-1999g, no significant OR proc + oth problem

714 Neonate, adm weight 1500-1999g, no significant OR proc, no problem

715 Neonate, adm weight 2000-2499g + significant OR procedure

717 Neonate, adm weight 2000-2499g, no significant OR proc + mult maj prob

718 Neonate, adm weightt 2000-2499g, no significant OR proc + maj problem

719 Neonate, adm weight 2000-2499g, no significant OR proc + oth problem

720 Neonate, adm weight 2000-2499g, no significant OR proc, no problem

721 Neonate, adm weight $>2499 g$ + significant OR proc + mult maj problems

722 Neonate, adm weight $>2499$ g + significant OR proc, no mult maj problems

723 Neonate, adm weight $>2499 \mathrm{~g}+$ minor abdominal problem

724 Neonate, adm weight $>2499$ g, no significant OR proc + mult maj problems
1503

1503

1503

1504

1504

1503

1503

1503

1503

1507

1505

1505

1505

1506

1507

1505

1505

1506

1505
PREMATURITY W MAJOR PROBLEMS

PREMATURITY WO MAJOR PROBLEMS

\section{NORMAL NEWBORN}

FULL TERM NEONATE W MAJOR PROBLEMS

NEONATE W OTHER SIGNIFICANT PROBLEMS 
726 Neonate, adm weight $>2499$ g, no significant OR proc + other problems

727 Neonate, adm weight $>2499$ g, no significant OR proc, no problem

750 Splenectomy

1507

1601

1603

1607

1607

1607

1604

1604

1604

1606

1606

1708

1707

1707

1711

1711

1701

1701

1704

1704

1704

1702

1702

1702

\section{SPLENECTOMY AGE $>17$}

OTHER O.R. PROCEDURES OF THE BLOOD AND BLOOD FORMING ORGANS RETICULOENDOTHELIAL \& IMMUNITY DISORDERS W CC

RETICULOENDOTHELIAL \& IMMUNITY DISORDERS WO CC

RED BLOOD CELL DISORDERS AGE $>17$

\section{COAGULATION DISORDERS}

CHEMOTHERAPY WO ACUTE LEUKEMIA AS SECONDARY DIAGNOSIS RADIOTHERAPY

OTHER MYELOPROLIF DIS OR POORLY DIFF NEOPL DIAG W CC OTHER MYELOPROLIF DIS OR POORLY DIFF NEOPL DIAG WO CC LYMPHOMA \& LEUKEMIA W MAJOR O.R. PROCEDURE (NO LONGER VALID)

ACUTE LEUKEMIA WO MAJOR O.R. PROCEDURE AGE 0-17

LYMPHOMA \& NON-ACUTE LEUKEMIA W OTHER O.R. PROC W CC

LYMPHOMA \& NON-ACUTE LEUKEMIA W OTHER O.R. PROC WO CC

CCmphoma and non-acute leukaemia + other OR procedure + non-major CC

792 Lymphoma and non-acute leukaemia + other OR procedure, no CC 
Other neoplastic disorders + major OR procedure + CC

796

Other neoplastic disorders + major OR procedure, no CC

HIV-related central nervous system disease

HIV-related malignancy

803 HIV-related infection

804 HIV + other related condition

805 HIV, no specified related condition

808 Septicaemia age $>34$

809 Septicaemia age $<35$

811 Fever of unknown origin age $>9+\mathrm{CC}$

812 Fever of unknown origin age $>9$, no CC

813 Fever of unknown origin age $<10$

814 Viral illness age $>59$

815 Viral illness age $<60$

816 Other infectious and parasitic diseases age $>49$

817 Other infectious and parasitic diseases age $<50$

818 OR proc for infectious parasitic diseases age $>54+C C$

819 OR proc for infectious and parasitic diseases age ( $>54$ no CC) or $(<55+\mathrm{CC})$

820 OR proc for infectious and parasitic diseases age $<55$, no CC

821 Postoperative and post-traumatic infections age $>54$

822 Postoperative and post-traumatic infections age $<55$

841 Schizophrenia disorders

842 Paranoia and acute psychotic disorders

843 Major affective disorders

844 Other affective and somatoform disorders

845 Anxiety disorders

1801 1801

1801

1804

1804

1909

1909

1909

1909

1909
LYMPHOMA \& NON-ACUTE LEUKEMIA W CC LYMPHOMA \& NON-ACUTE LEUKEMIA WO CC MYELOPROLIF DISORD OR POORLY DIFF NEOPL W MAJ O.R.PROC W CC MYELOPROLIF DISORD OR POORLY DIFF NEOPL W MAJ O.R.PROC WO CC MYELOPROLIF DISORD OR POORLY DIFF NEOPL W OTHER O.R.PROC HIV W MAJOR RELATED CONDITION

HIV W OR WO OTHER RELATED CONDITION

\section{SEPTICEMIA AGE >17}

FEVER OF UNKNOWN ORIGIN AGE $>17 \mathrm{~W}$ CC FEVER OF UNKNOWN ORIGIN AGE $>17$ WO CC VIRAL ILLNESS \& FEVER OF UNKNOWN ORIGIN AGE 0-17 VIRAL ILLNESS AGE $>17$

OTHER INFECTIOUS \& PARASITIC DISEASES DIAGNOSES

O.R. PROCEDURE FOR INFECTIOUS \& PARASITIC DISEASES
POSTOPERATIVE \& POST-TRAUMATIC INFECTIONS

OTHER MENTAL DISORDER DIAGNOSES 
860 Alcohol intoxication and withdrawal

861 Drug intoxication and withdrawal

862 Alcohol use disorder and dependence trauma

874 Other OR procedure for multiple significant trauma

CHILDHOOD MENTAL DISORDERS

ALCOHOL/DRUG ABUSE OR DEPENDENCE, LEFT AMA

\section{VALID)}

ALC/DRUG ABUSE OR DEPEND, DETOX OR OTH SYMPT TREAT WO CC (NO LONGER

VALID)

ALC/DRUG DEPENDENCE W REHABILITATION THERAPY (NO LONGER VALID)

ALC/DRUG DEPENDENCE, COMBINED REHAB \& DETOX THERAPY (NO LONGER

VALID)

ALCOHOL/DRUG ABUSE OR DEPENDENCE W CC

ALC/DRUG ABUSE OR DEPEND W REHABILITATION THERAPY WO CC

ALC/DRUG ABUSE OR DEPEND WO REHABILITATION THERAPY WO CC

TRAUMATIC INJURY AGE >17 W CC

TRAUMATIC INJURY AGE 0-17

\section{CRANIOTOMY FOR MULTIPLE SIGNIFICANT TRAUMA}

LIMB REATTACHMENT, HIP AND FEMUR PROC FOR MULTIPLE SIGNIFICANT TRA

OTHER O.R. PROCEDURES FOR MULTIPLE SIGNIFICANT TRAUMA

TRAUMATIC INJURY AGE >17 WO CC

OTHER MULTIPLE SIGNIFICANT TRAUMA

2107

2109

2109

2111

2111

2112

\section{ALLERGIC REACTIONS AGE $>17$}

POISONING \& TOXIC EFFECTS OF DRUGS AGE $>17 \mathrm{~W}$ CC

POISONING \& TOXIC EFFECTS OF DRUGS AGE $>17$ WO CC

COMPLICATIONS OF TREATMENT W CC

COMPLICATIONS OF TREATMENT WO CC

OTHER INJURY, POISONING \& TOXIC EFFECT DIAG W CC 
$\mathrm{CC}$

Other injury, poisoning and toxic effect diagnoses age $<60$, no CC Lead poisoning

895 Skin grafts for injuries to lower limb

896 Skin grafts for injuries to hand

897 Skin grafts for other injuries

898 Other procedures for injuries to lower limb age $>59$ or + CC

899 Other procedures for injuries to lower limb age $<60$ no CC

900 Other procedures for injuries to hand

901 Other procedures for other injuries + CC

902 Other procedures for other injuries, no CC

920 Burns, transferred to other acute facility, LOS $<5$ days

921 Severe third degree burns with skin graft

922 Other third degree burns with skin graft age $>64$

923 Other third degree burns with skin graft age $<65$

924 Other burns with skin graft

925 Other operating room procedures for burns

926 Severe burns

927 Other burns age $>64$

928 Other burns age $<65$

930 OR procedure + diagnosis of other contacts with health service + $\mathrm{CC}$

931 OR procedure + diagnosis of other contact with health service, no

932 Signs and symptoms
OTHER INJURY, POISONING \& TOXIC EFFECT DIAG WO CC

2101

2101

2101

2104

2104

2103

2104

2104

2201

2206

2202

2202

2202

2203

2207

2210

2210

2301

2301

SIGNS \& SYMPTOMS W CC

SIGNS \& SYMPTOMS WO CC

OTHER O.R. PROCEDURES FOR INJURIES W CC

OTHER O.R. PROCEDURES FOR INJURIES WO CC

HAND PROCEDURES FOR INJURIES

EXTENSIVE BURNS WO O.R. PROCEDURE (NO LONGER VALID)

EXTENSIVE 3RD DEGREE BURNS W SKIN GRAFT

NON-EXTENSIVE BURNS W SKIN GRAFT (NO LONGER VALID) LONGER VALID)

EXTENSIVE 3RD DEGREE BURNS WO SKIN GRAFT

NON-EXTENSIVE BURNS W CC OR SIGNIFICANT TRAUMA

NON-EXTENSIVE BURNS WO CC OR SIGNIFICANT TRAUMA

O.R. PROC W DIAGNOSES OF OTHER CONTACT W HEALTH SERVICES

934 Short stay contacts with health services

935 Multiple, other and unspecified congenital anomalies

BURNS, TRANSFERRED TO ANOTHER ACUTE CARE FACILITY (NO LONGER VALID)

NON-EXTENSIVE BURNS W WOUND DEBRIDEMENT OR OTHER O.R. PROC (NO 
Aftercare + secondary diagnosis of history of malignancy + endoscopy

937 Aftercare + secondary diagnosis of history of malignancy, no endoscopy

938 Same day aftercare, no secondary diagnosis of history of malignancy

939 Aftercare, no secondary diagnosis of history of malignancy

940 Same day rehabilitation

941 Rehabilitation

942 Other factors influencing health status age $>79$ or + CC

943 Other factors influencing health status age $<80$, no CC

950 Extensive OR procedure unrelated to principal diagnosis

951 Unacceptable obstetric diagnosis

952 Ungroupable

953 Prostatic OR procedure unrelated to principal diagnosis

954 Non-extensive OR procedure unrelated to principal diagnosis

955 Neonatal diagnosis not consistent with age or weight

956 Unacceptable principal diagnosis AFTERCARE WO HISTORY OF MALIGNANCY AS SECONDARY DIAGNOSIS

2302

2306

2306

8801

8803

8804

8805
REHABILITATION

OTHER FACTORS INFLUENCING HEALTH STATUS

EXTENSIVE O.R. PROCEDURE UNRELATED TO PRINCIPAL DIAGNOSIS

UNGROUPABLE

PROSTATIC O.R. PROCEDURE UNRELATED TO PRINCIPAL DIAGNOSIS

NON-EXTENSIVE O.R. PROCEDURE UNRELATED TO PRINCIPAL DIAGNOSIS 
Table 15: Unmatched AHRQ DRGs

\begin{tabular}{|c|c|}
\hline $\begin{array}{l}\text { AHRQ } \\
\text { DRG }\end{array}$ & Description \\
\hline 102 & CRANIOTOMY AGE 0-17 \\
\hline 107 & SPINAL DISORDERS \& INJURIES \\
\hline 111 & INTRACRANIAL HEMORRHAGE \& STROKE W INFARCT \\
\hline 112 & NONSPECIFIC CVA \& PRECEREBRAL OCCLUSION WO INFARCT \\
\hline 117 & HYPERTENSIVE ENCEPHALOPATHY \\
\hline 120 & SEIZURE \& HEADACHE AGE 0-17 \\
\hline 121 & TRAUMATIC STUPOR \& COMA, COMA >1 HR \\
\hline 122 & TRAUMATIC STUPOR \& COMA, COMA $<1$ HR AGE $>17$ W CC \\
\hline 122 & TRAUMATIC STUPOR \& COMA, COMA $<1$ HR AGE $>17$ WO CC \\
\hline 123 & TRAUMATIC STUPOR \& COMA, COMA $<1$ HR AGE 0-17 \\
\hline 124 & CONCUSSION AGE >17 W CC \\
\hline 124 & CONCUSSION AGE >17 WO CC \\
\hline 125 & CONCUSSION AGE 0-17 \\
\hline 128 & INTRACRANIAL VASCULAR PROC W PDX HEMORRHAGE \\
\hline 130 & ACUTE ISCHEMIC STROKE WITH USE OF THROMBOLYTIC AGENT \\
\hline 203 & PRIMARY IRIS PROCEDURES \\
\hline 205 & EXTRAOCULAR PROCEDURES EXCEPT ORBIT AGE >17 \\
\hline 206 & EXTRAOCULAR PROCEDURES EXCEPT ORBIT AGE 0-17 \\
\hline 207 & INTRAOCULAR PROCEDURES EXCEPT RETINA, IRIS \& LENS \\
\hline 210 & NEUROLOGICAL EYE DISORDERS \\
\hline 212 & OTHER DISORDERS OF THE EYE AGE 0-17 \\
\hline 306 & SINUS \& MASTOID PROCEDURES AGE 0-17 \\
\hline 309 & T\&A PROC, EXCEPT TONSILLECTOMY \&/OR ADENOIDECTOMY ONLY, AGE >17 \\
\hline 310 & T\&A PROC, EXCEPT TONSILLECTOMY \&/OR ADENOIDECTOMY ONLY, AGE 0-17 \\
\hline 312 & TONSILLECTOMY \&/OR ADENOIDECTOMY ONLY, AGE 0-17 \\
\hline 314 & MYRINGOTOMY W TUBE INSERTION AGE 0-17 \\
\hline 325 & OTHER EAR, NOSE, MOUTH \& THROAT DIAGNOSES AGE 0-17 \\
\hline
\end{tabular}




\begin{tabular}{|c|c|}
\hline 328 & DENTAL \& ORAL DIS EXCEPT EXTRACTIONS \& RESTORATIONS, AGE 0-17 \\
\hline 405 & RESPIRATORY INFECTIONS \& INFLAMMATIONS AGE 0-17 \\
\hline 411 & SIMPLE PNEUMONIA \& PLEURISY AGE >17 W CC \\
\hline 411 & SIMPLE PNEUMONIA \& PLEURISY AGE >17 WO CC \\
\hline 412 & SIMPLE PNEUMONIA \& PLEURISY AGE 0-17 \\
\hline 416 & BRONCHITIS \& ASTHMA AGE 0-17 \\
\hline 419 & NO LONGER VALID \\
\hline 503 & CORONARY BYPASS W PTCA \\
\hline 512 & OTHER PERMANENT CARDIAC PACEMAKER IMPLANT (NO LONGER VALID) \\
\hline 512 & OTHER PERMANENT CARDIAC PACEMAKER IMPLANT W \\
\hline 522 & CARDIAC ARREST, UNEXPLAINED \\
\hline 527 & CARDIAC CONGENITAL \& VALVULAR DISORDERS AGE 0-17 \\
\hline 535 & OTHER VASCULAR PROCEDURES W CC \\
\hline 535 & OTHER VASCULAR PROCEDURES WO CC \\
\hline 535 & OTHER VASCULAR PROCEDURES W CC W MAJOR CV DX \\
\hline 535 & OTHER VASCULAR PROCEDURES W CC W \\
\hline 536 & CARDIAC DEFIB IMPLANT W CARDIAC CATH (NO LONGER VALID) \\
\hline 538 & PERCUTANEOUS CARDIOVASC PROC W AMI \\
\hline 538 & PERCUTANEOUS CARDIOVASCULAR PROC W MAJOR CV DX \\
\hline 539 & PERC CARDIO PROC W NON-DRUG ELUTING STENT WO AMI \\
\hline 539 & PERCUTANEOUS CARDIOVASC PROC W NON-DRUG-ELUTING STENT W \\
\hline 540 & PERC CARDIO PROC WO CORONARY ARTERY STENT OR AMI \\
\hline 541 & HEART ASSIST SYSTEM IMPLANT \\
\hline 542 & PERCUTNEOUS CARDIOVASULAR PROC W DRUG ELUTING STENT W AMI \\
\hline 542 & PERCUTANEOUS CARDIOVASCULAR PROC W DRUG-ELUTING STENT W MAJOR CV DX \\
\hline 543 & PERCUTNEOUS CARDIOVASULAR PROC W DRUG ELUTING STENT WO AMI \\
\hline 543 & PERCUTANEOUS CARDIOVASCULAR PROC W DRUG-ELUTING STENT W \\
\hline 544 & CARDIAC DEFIB IMPLANT W CARDIAC CATH W AMI \\
\hline 544 & CARDIAC DEFIB IMPLANT W CARDIAC CATH WO AMI \\
\hline
\end{tabular}




\begin{tabular}{|c|c|}
\hline 606 & STOMACH, ESOPHAGEAL \& DUODENAL PROCEDURES AGE 0-17 \\
\hline 713 & LAPAROSCOPIC CHOLECYSTECTOMY WO C.D.E. W CC \\
\hline 713 & LAPAROSCOPIC CHOLECYSTECTOMY WO C.D.E. WO CC \\
\hline 820 & FRACTURES OF HIP \& PELVIS \\
\hline 833 & FX, SPRN, STRN \& DISL OF FOREARM, HAND, FOOT AGE 0-17 \\
\hline 835 & FX, SPRN, STRN \& DISL OF UPARM,LOWLEG EX FOOT AGE 0-17 \\
\hline 838 & MAJOR JOINT \& LIMB REATTACHMENT PROCEDURES OF UPPER EXTREMITY \\
\hline 839 & COMBINED ANTERIOR \\
\hline 842 & KNEE PROCEDURES W PDX OF INFECTION W CC \\
\hline 842 & KNEE PROCEDURES W PDX OF INFECTION WO CC \\
\hline 843 & KNEE PROCEDURES WO PDX OF INFECTION \\
\hline 844 & CERVICAL SPINAL FUSION W CC \\
\hline 844 & CERVICAL SPINAL FUSION WO CC \\
\hline 845 & LOCAL EXCIS \& REMOV OF INT FIX DEV EXCEPT HIP \& FEMUR W CC \\
\hline 845 & LOCAL EXCIS \& REMOV OF INT FIX DEV EXCEPT HIP \& FEMUR WO CC \\
\hline 846 & SPINAL FUSION EXC CERV WITH CURVATURE OF THE SPINE OR MALIG \\
\hline 903 & BREAST PROC FOR NON-MALIGNANCY EXCEPT BIOPSY \& LOCAL EXCISION \\
\hline 904 & BREAST BIOPSY \& LOCAL EXCISION FOR NON-MALIGNANCY \\
\hline 915 & CELLULITIS AGE 0-17 \\
\hline 917 & TRAUMA TO THE SKIN, SUBCUT TISS \& BREAST AGE 0-17 \\
\hline 1001 & AMPUTAT OF LOWER LIMB FOR ENDOCRINE,NUTRIT,\& METABOL DISORDERS \\
\hline 1003 & SKIN GRAFTS \& WOUND DEBRID FOR ENDOC, NUTRIT \& METAB DISORDERS \\
\hline 1012 & NUTRITIONAL \& MISC METABOLIC DISORDERS AGE 0-17 \\
\hline 1114 & KIDNEY \& URINARY TRACT INFECTIONS AGE 0-17 \\
\hline 1117 & KIDNEY \& URINARY TRACT SIGNS \& SYMPTOMS AGE 0-17 \\
\hline 1119 & URETHRAL STRICTURE AGE 0-17 \\
\hline 1121 & OTHER KIDNEY \& URINARY TRACT DIAGNOSES AGE 0-17 \\
\hline 1307 & LAPAROSCOPY \& INCISIONAL TUBAL INTERRUPTION \\
\hline 1309 & D\&C, CONIZATION \& RADIO-IMPLANT, FOR MALIGNANCY \\
\hline
\end{tabular}




\begin{tabular}{|c|c|}
\hline 1310 & D\&C, CONIZATION EXCEPT FOR MALIGNANCY \\
\hline 1403 & VAGINAL DELIVERY W STERILIZATION \& \\
\hline 1501 & NEONATES, DIED OR TRANSFERRED TO ANOTHER ACUTE CARE FACILITY \\
\hline 1602 & SPLENECTOMY AGE 0-17 \\
\hline 1605 & RED BLOOD CELL DISORDERS AGE 0-17 \\
\hline 1709 & HISTORY OF MALIGNANCY WO ENDOSCOPY \\
\hline 1710 & HISTORY OF MALIGNANCY W ENDOSCOPY \\
\hline 1712 & ACUTE LEUKEMIA WO MAJOR O.R. PROCEDURE AGE >17 \\
\hline 1713 & CHEMOTHERAPY W ACUTE LEUKEMIA OR W USE OF HI DOSE CHEMOAGENT \\
\hline 1714 & LYMPHOMA \& LEUKEMIA W MAJOR OR PROCEDURE W CC \\
\hline 1714 & LYMPHOMA \& LEUKEMIA W MAJOR OR PROCEDURE WO CC \\
\hline 1803 & SEPTICEMIA AGE 0-17 \\
\hline 1901 & O.R. PROCEDURE W PRINCIPAL DIAGNOSES OF MENTAL ILLNESS \\
\hline 1902 & ACUTE ADJUSTMENT REACTION \& PSYCHOSOCIAL DYSFUNCTION \\
\hline 1903 & DEPRESSIVE NEUROSES \\
\hline 1904 & NEUROSES EXCEPT DEPRESSIVE \\
\hline 1905 & DISORDERS OF PERSONALITY \& IMPULSE CONTROL \\
\hline 1906 & ORGANIC DISTURBANCES \& MENTAL RETARDATION \\
\hline 1907 & PSYCHOSES \\
\hline 2001 & NO LONGER VALID \\
\hline 2102 & WOUND DEBRIDEMENTS FOR INJURIES \\
\hline 2108 & ALLERGIC REACTIONS AGE 0-17 \\
\hline 2110 & POISONING \& TOXIC EFFECTS OF DRUGS AGE 0-17 \\
\hline 2204 & NON-EXTENSIVE BURNS WO O.R. PROCEDURE (NO LONGER VALID) \\
\hline 2205 & BILATERAL OR MULTIPLE MAJOR JOINT PROCS OF LOWER EXTREMITY (NO LONGER VALID) \\
\hline 2208 & FULL THICKNESS BURN W SKIN GRAFT OR INHAL INJ W CC OR SIG TRAUMA \\
\hline 2208 & FULL THICKNESS BURN W SKIN GRFT OR INHAL INJ WO CC OR SIG TRAUMA \\
\hline 2209 & FULL THICKNESS BURN WO SKIN GRFT OR INHAL INJ W CC OR SIG TRAUMA \\
\hline 2209 & FULL THICKNESS BURN WO SKIN GRFT OR INH INJ WO CC OR SIG TRAUMA \\
\hline
\end{tabular}




\section{Appendix C}

Table 16: PSI Unadjusted Rates Stratified by Age Category

\begin{tabular}{lrrrrrrrr}
\hline PSI & $<30$ & $\mathbf{3 0 - 3 9}$ & $\mathbf{4 0 - 4 9}$ & $\mathbf{5 0 - 5 9}$ & $\mathbf{6 0 - 6 9}$ & $\mathbf{7 0 - 7 9}$ & $\mathbf{8 0 +}$ & $\begin{array}{r}\text { Overall } \\
\text { Rate }\end{array}$ \\
\hline All & 11.50 & 10.45 & 12.04 & 15.00 & 20.05 & 23.77 & 23.70 & 16.44 \\
Gen & 1.38 & 2.11 & 2.66 & 2.58 & 3.14 & 3.43 & 2.49 & 2.63 \\
Med & 2.22 & 3.14 & 6.26 & 10.20 & 14.69 & 17.76 & 20.06 & 11.81 \\
Post & 10.82 & 12.95 & 17.18 & 21.38 & 27.77 & 31.51 & 29.13 & 22.24 \\
Obst & 15.86 & 15.76 & 7.48 & 52.63 &. &. &. &. \\
\hline PSI1 & 0.01 & 0.02 & 0.00 & 0.02 & 0.01 & 0.01 & 0.03 & 15.69 \\
PSI2 & 0.19 & 0.28 & 0.48 & 0.70 & 1.15 & 1.95 & 4.84 & 0.01 \\
PSI3 & 4.48 & 3.92 & 6.91 & 9.05 & 12.18 & 18.66 & 30.53 & 17.49 \\
PSI4 & 29.27 & 42.54 & 65.26 & 95.32 & 116.58 & 147.60 &. & 103.65 \\
PSI5 & 0.05 & 0.14 & 0.10 & 0.06 & 0.09 & 0.08 & 0.03 & 0.08 \\
PSI6 & 0.24 & 0.26 & 0.28 & 0.35 & 0.47 & 0.52 & 0.31 & 0.37 \\
PSI7 & 2.62 & 3.71 & 4.95 & 6.38 & 6.20 & 5.44 & 4.30 & 4.94 \\
PSI8 & 0.03 & 0.00 & 0.04 & 0.05 & 0.12 & 0.62 & 2.62 & 0.46 \\
PSI9 & 9.66 & 11.33 & 14.73 & 17.81 & 22.98 & 26.43 & 24.57 & 18.78 \\
PSI10 & 0.64 & 0.90 & 1.31 & 2.36 & 2.60 & 2.36 & 1.19 & 1.80 \\
PSI11 & 0.75 & 0.83 & 0.90 & 1.36 & 2.62 & 1.90 & 1.37 & 1.42 \\
PSI12 & 1.05 & 1.55 & 2.24 & 3.16 & 4.40 & 4.67 & 4.37 & 3.19 \\
PSI13 & 12.31 & 10.04 & 9.30 & 11.18 & 15.70 & 13.69 & 13.35 & 12.80 \\
PSI14 & 1.57 & 2.06 & 2.53 & 5.22 & 6.30 & 7.06 & 5.87 & 4.70 \\
PSI15 & 1.40 & 2.23 & 2.34 & 2.18 & 2.57 & 2.73 & 1.74 & 2.23 \\
PSI16 & 0.17 & 0.14 & 0.21 & 0.06 & 0.07 & 0.10 & 0.03 & 0.09 \\
PSI17 & 14.99 &. &. &. &. &. &. & 14.99 \\
PSI18 & 67.10 & 60.28 & 30.78 & 500.00 &. &. &. & 62.69 \\
PSI19 & 15.22 & 13.81 & 6.51 & 0.00 &. &. &. & 14.36 \\
PSI20 & 3.29 & 4.57 & 4.26 & 0.00 &. &. &. & 4.06 \\
\hline & & & & & & & & \\
\hline
\end{tabular}


Table 17: PSI Unadjusted Rates Stratified by Ethnicity

\begin{tabular}{lrrrrrr}
\hline PSI & NZ Euro & Maori & Pacific & Asian & Other & $\begin{array}{r}\text { Overall } \\
\text { Rate }\end{array}$ \\
\hline All & 17.11 & 12.62 & 15.36 & 19.20 & 17.35 & 16.44 \\
Gen & 2.75 & 2.13 & 2.05 & 2.68 & 2.77 & 2.63 \\
Med & 12.32 & 11.36 & 9.78 & 6.63 & 11.91 & 11.81 \\
Post & 22.29 & 22.14 & 24.52 & 20.53 & 22.39 & 22.24 \\
Obst & 14.82 & 9.25 & 16.39 & 32.25 & 17.98 & 15.69 \\
\hline PSI1 & 0.02 & 0.02 & 0.00 & 0.00 & 0.02 & 0.01 \\
PSI2 & 1.35 & 0.97 & 0.82 & 0.50 & 1.14 & 1.21 \\
PSI3 & 18.65 & 13.68 & 12.45 & 8.19 & 17.60 & 17.49 \\
PSI4 & 102.07 & 112.15 & 94.54 & 103.59 & 103.37 & 103.65 \\
PSI5 & 0.08 & 0.08 & 0.09 & 0.10 & 0.06 & 0.08 \\
PSI6 & 0.37 & 0.40 & 0.28 & 0.33 & 0.34 & 0.37 \\
PSI7 & 4.91 & 4.80 & 4.67 & 4.36 & 5.48 & 4.94 \\
PSI8 & 0.53 & 0.14 & 0.14 & 0.29 & 0.58 & 0.46 \\
PSI9 & 18.65 & 19.25 & 21.52 & 18.61 & 18.61 & 18.78 \\
PSI10 & 1.54 & 3.02 & 3.50 & 1.49 & 1.54 & 1.80 \\
PSI11 & 1.43 & 1.48 & 0.68 & 2.36 & 1.45 & 1.42 \\
PSI12 & 3.41 & 2.32 & 2.57 & 1.78 & 3.59 & 3.19 \\
PSI13 & 12.80 & 15.11 & 19.67 & 14.99 & 8.63 & 12.80 \\
PSI14 & 4.71 & 5.29 & 4.70 & 3.38 & 4.58 & 4.70 \\
PSI15 & 2.31 & 1.79 & 1.84 & 2.56 & 2.36 & 2.23 \\
PSI16 & 0.09 & 0.08 & 0.07 & 0.31 & 0.05 & 0.09 \\
PSI17 & 12.91 & 8.48 & 19.57 & 32.62 & 19.42 & 14.99 \\
PSI18 & 59.67 & 47.44 & 60.69 & 93.93 & 56.83 & 62.69 \\
PSI19 & 14.67 & 9.12 & 12.48 & 30.07 & 15.33 & 14.36 \\
PSI20 & 4.30 & 3.18 & 4.16 & 4.72 & 3.37 & 4.06 \\
\hline & & & & & &
\end{tabular}


Table 18: Unadjusted Rates Stratified by NZDep

\begin{tabular}{lrrrrrr}
\hline PSI & Dep1\&2 & Dep3\&4 & Dep5\&6 & Dep7\&8 & Dep9\&10 & $\begin{array}{r}\text { Overall } \\
\text { Rate }\end{array}$ \\
\hline All & 16.67 & 17.04 & 17.09 & 16.67 & 15.27 & 16.44 \\
Gen & 2.63 & 2.83 & 2.73 & 2.71 & 2.35 & 2.63 \\
Med & 11.29 & 11.35 & 12.10 & 12.04 & 11.93 & 11.81 \\
Post & 22.53 & 22.93 & 22.58 & 22.07 & 21.52 & 22.24 \\
Obst & 16.63 & 17.06 & 16.44 & 15.60 & 14.03 & 15.69 \\
\hline PSI1 & 0.03 & 0.01 & 0.01 & 0.01 & 0.01 & 0.01 \\
PSI2 & 1.05 & 1.24 & 1.27 & 1.27 & 1.16 & 1.21 \\
PSI3 & 17.60 & 16.77 & 18.05 & 17.81 & 17.08 & 17.49 \\
PSI4 & 91.31 & 98.28 & 104.58 & 103.19 & 112.22 & 103.65 \\
PSI5 & 0.06 & 0.08 & 0.09 & 0.07 & 0.08 & 0.08 \\
PSI6 & 0.32 & 0.35 & 0.36 & 0.37 & 0.41 & 0.37 \\
PSI7 & 5.09 & 4.83 & 5.05 & 4.95 & 4.84 & 4.94 \\
PSI8 & 0.52 & 0.58 & 0.51 & 0.42 & 0.35 & 0.46 \\
PSI9 & 18.94 & 19.31 & 19.03 & 18.61 & 18.31 & 18.78 \\
PSI10 & 1.43 & 1.25 & 1.73 & 1.90 & 2.36 & 1.80 \\
PSI11 & 1.17 & 1.75 & 1.36 & 1.57 & 1.25 & 1.42 \\
PSI12 & 3.50 & 3.51 & 3.23 & 3.16 & 2.79 & 3.19 \\
PSI13 & 9.57 & 11.62 & 13.38 & 13.68 & 13.78 & 12.80 \\
PSI14 & 4.29 & 3.93 & 4.78 & 5.07 & 5.04 & 4.70 \\
PSI15 & 2.31 & 2.43 & 2.31 & 2.32 & 1.92 & 2.23 \\
PSI16 & 0.02 & 0.08 & 0.15 & 0.10 & 0.06 & 0.09 \\
PSI17 & 15.86 & 16.93 & 15.47 & 14.82 & 13.30 & 14.99 \\
PSI18 & 55.85 & 61.27 & 63.56 & 60.73 & 71.51 & 62.69 \\
PSI19 & 15.85 & 14.99 & 15.31 & 14.90 & 12.54 & 14.36 \\
PSI20 & 4.36 & 3.91 & 4.48 & 4.02 & 3.61 & 4.06 \\
\hline & & & & & &
\end{tabular}


Table 19: Unadjusted Rates Stratified by Major Diagnostic Category

\begin{tabular}{|c|c|c|c|c|c|c|c|c|c|c|c|c|c|}
\hline PSI & MDC1 & MDC2 & MDC3 & MDC4 & MDC5 & MDC6 & MDC7 & MDC8 & MDC9 & MDC10 & MDC11 & MDC12 & Total \\
\hline All & 15.93 & 4.26 & 9.64 & 16.53 & 22.30 & 17.06 & 26.45 & 19.64 & 10.19 & 25.35 & 11.35 & 28.31 & 16.44 \\
\hline General & 0.99 & 3.36 & 1.67 & 3.04 & 1.83 & 5.04 & 9.45 & 1.87 & 0.67 & 2.20 & 2.10 & 3.86 & 2.63 \\
\hline Medical & 15.85 & 1.51 & 4.27 & 15.86 & 11.89 & 8.46 & 11.80 & 11.45 & 3.65 & 23.97 & 14.95 & 7.79 & 11.81 \\
\hline Post-op & 27.83 & 0.85 & 11.87 & 23.12 & 66.69 & 24.83 & 26.28 & 17.18 & 11.24 & 30.18 & 31.96 & 35.90 & 22.24 \\
\hline Obstetric & & & & 0.00 & 0.00 & & & & & . & . & • & 15.69 \\
\hline PSI1 & 0.02 & 0.00 & 0.00 & 0.03 & 0.00 & 0.01 & 0.05 & 0.01 & 0.01 & 0.06 & 0.00 & 0.04 & 0.01 \\
\hline PSI2 & 1.62 & 0.13 & 0.67 & 2.00 & 1.65 & 1.00 & 1.21 & 1.83 & 0.54 & 1.42 & 1.93 & 0.32 & 1.21 \\
\hline PSI3 & 13.80 & 4.39 & 11.41 & 15.82 & 11.06 & 10.82 & 6.26 & 17.55 & & 34.98 & 20.36 & 13.68 & 17.49 \\
\hline PSI4 & 202.73 & 30.93 & 78.09 & 115.22 & 68.59 & 139.40 & 185.70 & 82.63 & 92.20 & 72.62 & 73.95 & 102.51 & 103.65 \\
\hline PSI5 & 0.03 & 0.02 & 0.05 & 0.01 & 0.05 & 0.08 & 0.26 & 0.10 & 0.05 & 0.04 & 0.09 & 0.11 & 0.08 \\
\hline PSI6 & 0.26 & 0.01 & 0.14 & 2.53 & 0.20 & 0.24 & 0.35 & 0.14 & 0.09 & 0.29 & 0.12 & 0.07 & 0.37 \\
\hline PSI7 & 5.34 & 1.19 & 2.47 & 3.32 & 9.71 & 5.80 & 4.49 & 2.35 & 5.43 & 5.47 & 2.75 & 0.77 & 4.94 \\
\hline PSI8 & 0.33 & 0.01 & 0.07 & 1.49 & 0.76 & 0.39 & 0.23 & . & 0.12 & 1.03 & 0.38 & 0.15 & 0.46 \\
\hline PSI9 & 23.86 & 0.78 & 11.36 & 14.04 & 60.96 & 19.14 & 22.41 & 12.48 & 10.59 & 26.78 & 28.60 & 34.74 & 18.78 \\
\hline PSI10 & 1.16 & 0.23 & 0.19 & 2.36 & 3.14 & 1.44 & 6.23 & 0.55 & 0.43 & 1.15 & 3.43 & 0.00 & 1.80 \\
\hline PSI11 & 3.18 & 0.00 & 0.00 & $\cdot$ & $\cdot$ & 3.61 & 7.15 & 0.55 & 0.33 & 1.73 & 1.22 & 0.00 & 1.42 \\
\hline PSI12 & 3.56 & 0.06 & 0.57 & 8.53 & 5.60 & 4.25 & 3.39 & 4.90 & 0.71 & 2.82 & 2.98 & 1.50 & 3.19 \\
\hline PSI13 & 16.69 & 0.00 & 0.00 & 21.47 & 9.70 & 36.23 & 34.60 & 3.50 & 13.28 & 23.48 & 22.06 & 9.17 & 12.80 \\
\hline PSI14 & 9.21 & 0.00 & 40.82 & 20.49 & 6.93 & 4.34 & 2.63 & 20.10 & 3.08 & 4.25 & 6.45 & 0.90 & 4.70 \\
\hline PSI15 & 0.67 & 3.32 & 1.44 & 0.91 & 1.47 & 4.65 & 8.77 & 1.62 & 0.46 & 1.63 & 1.85 & 3.55 & 2.23 \\
\hline PSI16 & 0.00 & 0.00 & 0.00 & 0.00 & 0.24 & 0.05 & 0.00 & 0.08 & 0.00 & 0.00 & 0.00 & 0.27 & 0.09 \\
\hline PSI17 & . & . & . & . & . & . & . & . & $\cdot$ & . & . & . & 14.99 \\
\hline PSI18 & . & . & . & . & . & . & . & . & . & . & . & . & 62.69 \\
\hline PSI19 & . & . & . & . & . & . & . & . & . & . & . & . & 14.36 \\
\hline PSI20 & . & . & . & . & . & . & . & . & . & . & . & . & 4.06 \\
\hline
\end{tabular}


Table 19: Unadjusted Rates Stratified by Major Diagnostic Category (continued)

\begin{tabular}{|c|c|c|c|c|c|c|c|c|c|c|c|c|}
\hline PSI & MDC13 & MDC14 & MDC15 & MDC16 & MDC17 & MDC18 & MDC19 & MDC20 & MDC21 & MDC22 & MDC23 & Total \\
\hline All & 20.52 & 11.30 & 14.99 & 6.51 & 9.08 & 37.73 & 3.13 & 4.67 & 18.22 & 21.10 & 28.69 & 16.44 \\
\hline General & 9.10 & 0.03 & . & 0.86 & 1.00 & 2.18 & 0.12 & 0.16 & 2.00 & 1.77 & 2.36 & 2.63 \\
\hline Medical & 2.61 & 0.10 & . & 7.70 & 19.47 & 33.33 & 2.93 & 5.14 & 9.61 & 19.60 & 32.99 & 11.81 \\
\hline Post-op & 13.42 & . & . & 34.74 & 36.53 & 81.07 & 2.49 & 0.00 & 35.02 & 21.06 & 21.64 & 22.24 \\
\hline Obstetric & . & . & 14.99 & 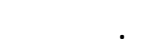 & & . & . & . & ${ }^{\circ}$ & . & 0.00 & 15.69 \\
\hline PSI1 & 0.00 & 0.03 & . & 0.00 & 0.00 & 0.00 & 0.00 & 0.00 & 0.09 & 0.00 & 0.00 & 0.01 \\
\hline PSI2 & 0.35 & 0.06 & . & 1.70 & 0.83 & 1.57 & 1.39 & 0.75 & 2.01 & . & 2.27 & 1.21 \\
\hline PSI3 & 4.09 & . & . & 11.43 & 14.06 & 20.97 & 2.26 & 5.99 & 18.00 & 11.16 & 44.52 & 17.49 \\
\hline PSI4 & 69.82 & 5.55 & . & 47.96 & 195.19 & 223.05 & 29.89 & 52.24 & 62.08 & 144.44 & 50.24 & 103.65 \\
\hline PSI5 & 0.27 & . & . & 0.00 & 0.02 & 0.30 & 0.00 & 0.00 & 0.12 & 0.00 & 0.06 & 0.08 \\
\hline PSI6 & 0.03 & . & . & 0.11 & 0.19 & 0.49 & 0.07 & 0.16 & 0.66 & 0.90 & 0.66 & 0.37 \\
\hline PSI7 & 1.55 & . & . & 6.27 & 8.18 & 8.54 & 0.48 & 3.16 & 4.06 & 10.32 & 3.49 & 4.94 \\
\hline PSI8 & 0.02 & . & . & 2.00 & 0.94 & 1.62 & 1.81 & 0.00 & 0.52 & . & 12.24 & 0.46 \\
\hline PSI9 & 12.03 & . & . & 25.55 & 22.82 & 66.97 & 0.77 & 0.00 & 31.86 & 16.69 & 13.29 & 18.78 \\
\hline PSI10 & 0.42 & . & . & 1.32 & 2.65 & 22.16 & 0.00 & 0.00 & 0.75 & 2.57 & 1.51 & 1.80 \\
\hline PSI11 & 0.14 & . & . & 0.00 & 1.69 & 15.52 & 0.00 & 0.00 & 3.99 & 0.00 & 0.57 & 1.42 \\
\hline PSI12 & 1.03 & . & . & 8.75 & 12.62 & 10.95 & 1.73 & 0.00 & 4.45 & 3.97 & 6.50 & 3.19 \\
\hline PSI13 & 4.86 & . & . & 20.41 & 0.00 & 32.26 & 0.00 & 0.00 & 24.36 & 10.58 & 31.28 & 12.80 \\
\hline PSI14 & 3.07 & . & . & 3.06 & 4.89 & 14.06 & 0.00 & 0.00 & 27.15 & 0.00 & 3.27 & 4.70 \\
\hline PSI15 & 8.79 & . & . & 0.61 & 0.77 & 1.17 & 0.04 & 0.00 & 1.16 & 1.06 & 1.01 & 2.23 \\
\hline PSI16 & 0.00 & . & . & 0.07 & 0.10 & 0.32 & 0.00 & 0.00 & 0.00 & 0.00 & 0.32 & 0.09 \\
\hline PSI17 & . & . & 14.99 & . & . & . & . & . & . & . & . & 14.99 \\
\hline PSI18 & . & 62.69 & . & . & . & . & . & . & . & . & . & 62.69 \\
\hline PSI19 & . & 14.36 & . & . & . & . & . & . & . & . & . & 14.36 \\
\hline PSI20 & . & 4.06 & . & . & . & . & . & . & . & . & . & 4.06 \\
\hline
\end{tabular}


Table 20: Unadjusted Rates Stratified by Comorbidities

\begin{tabular}{|c|c|c|c|c|c|c|c|c|c|c|c|c|c|c|c|}
\hline Comorbidity & & & 3 & & 4 & + & 5 & & $\epsilon$ & & & & & & \\
\hline PSI & 0 & 1 & 0 & 1 & 0 & 1 & 0 & 1 & 0 & 1 & 0 & 1 & 0 & 1 & Total \\
\hline All & 15.39 & 51.68 & 16.05 & 44.28 & 15.89 & 150.58 & 15.76 & 63.78 & 14.74 & 30.72 & 16.31 & 22.47 & 16.16 & 32.85 & 16.44 \\
\hline General & 2.59 & 3.53 & 2.61 & 3.71 & 2.61 & 5.73 & 2.58 & 5.12 & 2.52 & 3.38 & 2.64 & 2.12 & 2.64 & 1.97 & 2.63 \\
\hline Medical & 10.28 & 45.09 & 11.48 & 27.40 & 11.46 & 64.72 & 11.06 & 49.37 & 10.34 & 20.16 & 11.65 & 17.45 & 11.29 & 31.98 & 11.81 \\
\hline Post-op & 21.16 & 90.38 & 21.36 & 109.25 & 20.61 & 569.88 & 21.22 & 81.21 & 18.58 & 54.11 & 21.81 & 68.05 & 22.07 & 45.71 & 22.24 \\
\hline Obstetric & 15.68 & 32.47 & 15.68 & 31.02 & 15.69 & 43.96 & 15.69 & 23.81 & 15.69 & 16.26 & 15.69 & 26.32 & 15.68 & 22.42 & 15.69 \\
\hline PSI1 & 0.01 & 0.09 & 0.01 & 0.00 & 0.01 & 0.23 & 0.01 & 0.04 & 0.01 & 0.02 & 0.01 & 0.07 & 0.01 & 0.00 & 0.01 \\
\hline PSI2 & 1.00 & 17.41 & 1.16 & 5.53 & 1.18 & 18.87 & 1.16 & 7.29 & 1.04 & 2.69 & 1.15 & 11.31 & 1.13 & 10.11 & 1.21 \\
\hline PSI3 & 15.89 & 32.77 & 17.39 & 19.79 & 17.39 & 23.73 & 16.17 & 49.80 & 16.97 & 19.28 & 17.48 & 60.61 & 16.79 & 36.81 & 17.49 \\
\hline PSI4 & 90.60 & 219.04 & 101.94 & 148.68 & 101.30 & 137.40 & 100.36 & 182.26 & 100.96 & 112.08 & 99.31 & 166.93 & 100.09 & 181.54 & 103.65 \\
\hline PSI5 & 0.08 & 0.04 & 0.08 & 0.09 & 0.08 & 0.17 & 0.08 & 0.13 & 0.07 & 0.09 & 0.08 & 0.07 & 0.08 & 0.03 & 0.08 \\
\hline PSI6 & 0.35 & 0.75 & 0.36 & 0.56 & 0.36 & 1.78 & 0.36 & 0.62 & 0.36 & 0.39 & 0.37 & 0.43 & 0.37 & 0.41 & 0.37 \\
\hline PSI7 & 4.73 & 8.31 & 4.80 & 9.92 & 4.90 & 10.00 & 4.82 & 9.79 & 4.38 & 7.37 & 4.92 & 5.35 & 4.91 & 5.85 & 4.94 \\
\hline PSI8 & 0.39 & 4.92 & 0.44 & 2.54 & 0.45 & 2.96 & 0.43 & 1.79 & 0.37 & 1.18 & 0.45 & 2.14 & 0.42 & 7.50 & 0.46 \\
\hline PSI9 & 17.98 & 68.95 & 17.97 & 98.44 & 18.52 & 104.18 & 17.88 & 70.41 & 15.61 & 46.36 & 18.44 & 54.23 & 18.65 & 36.80 & 18.78 \\
\hline PSI10 & 1.42 & 17.08 & 1.61 & 11.72 & 1.66 & 28.32 & 1.63 & 6.20 & 1.07 & 5.79 & 1.77 & 4.03 & 1.80 & 2.01 & 1.80 \\
\hline PSI11 & 1.16 & 21.42 & 1.33 & 14.54 & 1.36 & 25.53 & 1.35 & 5.02 & 1.15 & 3.84 & 1.34 & 6.20 & 1.41 & 2.54 & 1.42 \\
\hline PSI12 & 2.97 & 16.94 & 3.13 & 9.42 & 1.68 & 517.89 & 3.08 & 9.68 & 2.76 & 6.95 & 3.10 & 12.30 & 3.15 & 8.74 & 3.19 \\
\hline PSI13 & 10.66 & 42.51 & 11.84 & 28.43 & 12.07 & 53.33 & 11.98 & 23.27 & 12.31 & 13.99 & 12.12 & 30.65 & 12.71 & 17.24 & 12.80 \\
\hline PSI14 & 4.40 & 15.80 & 4.63 & 10.61 & 4.67 & 8.82 & 4.59 & 10.12 & 4.12 & 8.83 & 4.64 & 9.90 & 4.66 & 8.25 & 4.70 \\
\hline PSI15 & 2.23 & 2.32 & 2.22 & 2.76 & 2.22 & 3.61 & 2.20 & 3.95 & 2.15 & 2.71 & 2.25 & 1.49 & 2.25 & 1.13 & 2.23 \\
\hline PSI16 & 0.08 & 0.10 & 0.08 & 0.29 & 0.09 & 0.00 & 0.08 & 0.10 & 0.08 & 0.10 & 0.08 & 0.17 & 0.08 & 0.20 & 0.09 \\
\hline PSI17 & 14.98 & 43.48 & 14.98 & 56.91 & 14.98 & 83.33 & 14.99 & 0.00 & 14.99 & & 14.99 & 0.00 & 14.98 & 32.26 & 14.99 \\
\hline PSI18 & 62.67 & 117.65 & 62.68 & 69.31 & 62.70 & 0.00 & 62.70 & 0.00 & 62.71 & 38.46 & 62.68 & 142.86 & 62.56 & 129.03 & 62.69 \\
\hline PSI19 & 14.36 & 22.22 & 14.35 & 28.65 & 14.36 & 0.00 & 14.36 & 0.00 & 14.36 & 20.00 & 14.36 & 34.48 & 14.36 & 13.13 & 14.36 \\
\hline PSI20 & 4.05 & 11.63 & 4.06 & 4.29 & 4.06 & 0.00 & 4.05 & 62.50 & 4.05 & 10.36 & 4.06 & 0.00 & 4.06 & 3.22 & 4.06 \\
\hline
\end{tabular}


Table 20: Unadjusted Rates Stratified by Comorbidities (continued)

\begin{tabular}{|c|c|c|c|c|c|c|c|c|c|c|c|c|c|c|c|}
\hline Comorbidity & & & 1 & & & & 1 & & 1 & & & & & & \\
\hline PSI & 0 & 1 & 0 & 1 & 0 & 1 & 0 & 1 & 0 & 1 & 0 & 1 & 0 & 1 & Total \\
\hline All & 15.89 & 39.16 & 16.26 & 21.28 & 15.76 & 32.88 & 16.40 & 31.58 & 16.03 & 22.86 & 16.20 & 55.06 & 16.41 & 45.35 & 16.44 \\
\hline General & 2.56 & 4.97 & 2.62 & 2.71 & 2.63 & 2.60 & 2.63 & 3.12 & 2.68 & 1.92 & 2.61 & 5.28 & 2.62 & 6.57 & 2.63 \\
\hline Medical & 11.06 & 32.08 & 11.67 & 14.57 & 10.70 & 36.70 & 11.76 & 23.07 & 10.61 & 50.10 & 11.46 & 57.47 & 11.78 & 32.56 & 11.81 \\
\hline Post-op & 21.67 & 60.46 & 21.90 & 31.38 & 21.42 & 44.80 & 22.17 & 62.08 & 21.01 & 65.76 & 21.96 & 87.16 & 22.20 & 89.87 & 22.24 \\
\hline Obstetric & 15.70 & 9.97 & 15.69 & 18.25 & 15.69 & 10.59 & 15.69 & 15.05 & 15.68 & 48.08 & 15.69 & 17.81 & 15.69 & 0.00 & 15.69 \\
\hline PSI1 & 0.01 & 0.05 & 0.01 & 0.02 & 0.01 & 0.02 & 0.01 & 0.00 & 0.01 & 0.03 & 0.01 & 0.16 & 0.01 & 0.00 & 0.01 \\
\hline PSI2 & 1.12 & 8.05 & 1.17 & 2.09 & 1.15 & 4.53 & 1.21 & 1.28 & 1.19 & 34.27 & 1.17 & 9.90 & 1.21 & 3.03 & 1.21 \\
\hline PSI3 & 16.97 & 24.43 & 17.64 & 15.37 & 16.14 & 32.62 & 17.41 & 26.29 & 15.94 & 33.17 & 17.44 & 21.10 & 17.46 & 26.89 & 17.49 \\
\hline PSI4 & 97.56 & 175.28 & 102.27 & 128.94 & 101.29 & 117.13 & 103.60 & 112.63 & 98.50 & 133.88 & 97.12 & 286.43 & 104.47 & 39.02 & 103.65 \\
\hline PSI5 & 0.08 & 0.04 & 0.07 & 0.11 & 0.07 & 0.10 & 0.08 & 0.06 & 0.08 & 0.06 & 0.08 & 0.11 & 0.08 & 0.00 & 0.08 \\
\hline PSI6 & 0.32 & 2.17 & 0.37 & 0.33 & 0.37 & 0.30 & 0.37 & 0.25 & 0.37 & 0.29 & 0.36 & 0.85 & 0.37 & 0.74 & 0.37 \\
\hline PSI7 & 4.89 & 5.99 & 4.93 & 5.20 & 4.80 & 7.84 & 4.94 & 4.83 & 4.93 & 9.65 & 4.90 & 10.16 & 4.93 & 13.97 & 4.94 \\
\hline PSI8 & 0.43 & 2.21 & 0.44 & 0.86 & 0.43 & 1.06 & 0.46 & 3.17 & 0.40 & 2.25 & 0.45 & 2.00 & 0.46 & 5.39 & 0.46 \\
\hline PSI9 & 18.38 & 45.06 & 18.50 & 26.14 & 18.10 & 37.23 & 18.72 & 47.37 & 17.76 & 54.55 & 18.56 & 70.78 & 18.75 & 69.23 & 18.78 \\
\hline PSI10 & 1.63 & 9.89 & 1.79 & 2.02 & 1.33 & 9.68 & 1.77 & 12.12 & 1.15 & 11.37 & 1.66 & 22.47 & 1.79 & 28.17 & 1.80 \\
\hline PSI11 & 1.17 & 21.82 & 1.38 & 2.56 & 1.25 & 5.04 & 1.40 & 10.81 & 1.30 & 3.47 & 1.32 & 14.33 & 1.40 & 33.90 & 1.42 \\
\hline PSI12 & 3.06 & 11.96 & 3.14 & 4.40 & 3.08 & 6.16 & 3.17 & 11.19 & 2.98 & 10.60 & 3.15 & 12.74 & 3.18 & 21.82 & 3.19 \\
\hline PSI13 & 11.94 & 30.77 & 12.57 & 16.26 & 12.42 & 17.98 & 12.70 & 28.17 & 12.69 & 55.56 & 12.25 & 74.87 & 12.77 & 28.57 & 12.80 \\
\hline PSI14 & 4.37 & 15.47 & 4.52 & 7.88 & 4.53 & 10.87 & 4.68 & 9.01 & 4.69 & 8.51 & 4.68 & 5.87 & 4.70 & 3.68 & 4.70 \\
\hline PSI15 & 2.21 & 2.77 & 2.23 & 2.17 & 2.24 & 2.02 & 2.23 & 2.57 & 2.30 & 1.38 & 2.21 & 4.11 & 2.23 & 5.33 & 2.23 \\
\hline PSI16 & 0.09 & 0.00 & 0.09 & 0.07 & 0.08 & 0.10 & 0.09 & 0.00 & 0.09 & 0.07 & 0.08 & 0.14 & 0.09 & 0.00 & 0.09 \\
\hline PSI17 & 14.99 & 0.00 & 14.99 & 0.00 & 14.99 & . & 14.99 & 0.00 & 14.98 & 76.92 & 14.98 & 166.67 & 14.99 & . & 14.99 \\
\hline PSI18 & 62.72 & 52.08 & 62.70 & 50.00 & 62.68 & 81.08 & 62.66 & 89.29 & 62.69 & 62.50 & 62.68 & 83.33 & 62.69 & . & 62.69 \\
\hline PSI19 & 14.37 & 11.96 & 14.36 & 34.88 & 14.37 & 10.75 & 14.37 & 5.08 & 14.35 & 71.43 & 14.37 & 9.09 & 14.36 & 0.00 & 14.36 \\
\hline PSI20 & 4.07 & 1.72 & 4.06 & 5.99 & 4.06 & 4.57 & 4.06 & 5.65 & 4.05 & 20.62 & 4.06 & 7.30 & 4.06 & 0.00 & 4.06 \\
\hline
\end{tabular}


Table 20: Unadjusted Rates Stratified by Comorbidities (continued)

\begin{tabular}{|c|c|c|c|c|c|c|c|c|c|c|c|c|c|}
\hline \multirow{2}{*}{$\begin{array}{l}\text { Comorbidity } \\
\text { PSI }\end{array}$} & \multicolumn{2}{|c|}{16} & \multicolumn{2}{|c|}{17} & \multicolumn{2}{|c|}{18} & \multicolumn{2}{|c|}{19} & \multicolumn{2}{|c|}{20} & \multicolumn{2}{|c|}{22} & \multirow[b]{2}{*}{ Total } \\
\hline & 0 & 1 & 0 & 1 & 0 & 1 & 0 & 1 & 0 & 1 & 0 & 1 & \\
\hline All & 16.44 & 21.00 & 16.44 & 16.45 & 15.91 & 38.83 & 16.28 & 27.62 & 16.35 & 31.43 & 16.17 & 34.93 & 16.44 \\
\hline General & 2.63 & 1.75 & 2.64 & 1.01 & 2.55 & 5.44 & 2.59 & 4.83 & 2.63 & 2.29 & 2.60 & 3.94 & 2.63 \\
\hline Medical & 11.81 & 51.64 & 11.70 & 55.16 & 11.11 & 53.39 & 11.57 & 38.16 & 11.67 & 29.96 & 11.52 & 26.73 & 11.81 \\
\hline Post-op & 22.24 & 20.98 & 22.20 & 50.90 & 21.43 & 55.29 & 21.87 & 51.32 & 22.14 & 43.83 & 21.81 & 52.24 & 22.24 \\
\hline Obstetric & 15.69 & 0.00 & 15.69 & 100.00 & 15.69 & 0.00 & 15.69 & 0.00 & 15.69 & 27.40 & 15.69 & 14.82 & 15.69 \\
\hline PSI1 & 0.01 & 0.00 & 0.01 & 0.00 & 0.01 & 0.00 & 0.01 & 0.00 & 0.01 & 0.00 & 0.01 & 0.05 & 0.01 \\
\hline PSI2 & 1.21 & . & 1.21 & . & 1.21 & . & 1.21 & & 1.20 & 4.09 & 1.19 & 2.57 & 1.21 \\
\hline PSI3 & 17.43 & 25.24 & 17.43 & 25.24 & 17.16 & 23.61 & 17.34 & 22.58 & 17.27 & 31.76 & 17.35 & 22.13 & 17.49 \\
\hline PSI4 & 102.35 & 198.95 & 102.35 & 198.95 & 93.67 & 225.88 & 101.94 & 154.32 & 103.36 & 121.21 & 102.60 & 120.64 & 103.65 \\
\hline PSI5 & 0.08 & 0.06 & 0.08 & 0.06 & 0.08 & 0.07 & 0.08 & 0.04 & 0.08 & 0.06 & 0.07 & 0.24 & 0.08 \\
\hline PSI6 & 0.37 & 0.38 & 0.37 & 0.38 & 0.35 & 0.83 & 0.36 & 1.03 & 0.37 & 0.47 & 0.37 & 0.27 & 0.37 \\
\hline PSI7 & 4.94 & . & 4.94 & . & 4.94 & . & 4.94 & 0.00 & 4.94 & 5.06 & 4.85 & 8.68 & 4.94 \\
\hline PSI8 & 0.46 & . & 0.46 & . & 0.46 & . & 0.45 & 1.10 & 0.45 & 2.47 & 0.46 & 0.25 & 0.46 \\
\hline PSI9 & 18.76 & 30.73 & 18.76 & 30.73 & 18.29 & 38.64 & 18.49 & 41.26 & 18.71 & 31.94 & 18.45 & 40.97 & 18.78 \\
\hline PSI10 & 1.79 & 6.07 & 1.79 & 6.07 & 1.83 & 1.09 & 1.79 & 2.27 & 1.76 & 9.94 & 1.65 & 10.57 & 1.80 \\
\hline PSI11 & 1.43 & 0.00 & 1.43 & 0.00 & 1.46 & 0.66 & 1.43 & 1.15 & 1.38 & 7.31 & 1.34 & 6.64 & 1.42 \\
\hline PSI12 & 3.16 & 21.41 & 3.16 & 21.41 & 2.88 & 15.73 & 3.11 & 9.88 & 3.16 & 10.42 & 3.11 & 8.70 & 3.19 \\
\hline PSI13 & 12.80 & . & 12.80 & . & 12.80 & . & 12.80 & & 12.67 & 23.90 & 12.78 & 13.37 & 12.80 \\
\hline PSI14 & 4.70 & 3.19 & 4.70 & 3.19 & 4.43 & 7.11 & 4.61 & 7.54 & 4.67 & 10.95 & 4.42 & 15.13 & 4.70 \\
\hline PSI15 & 2.24 & 0.59 & 2.24 & 0.59 & 2.16 & 4.65 & 2.20 & 3.73 & 2.23 & 1.56 & 2.21 & 3.48 & 2.23 \\
\hline PSI16 & 0.08 & 0.18 & 0.08 & 0.18 & 0.08 & 0.10 & 0.09 & 0.00 & 0.08 & 0.27 & 0.09 & 0.00 & 0.09 \\
\hline PSI17 & 14.99 & . & 14.99 & . & 14.99 & . & 14.99 & 0.00 & 14.99 & 0.00 & 14.99 & . & 14.99 \\
\hline PSI18 & 62.67 & 1000.00 & 62.67 & 1000.00 & 62.69 & . & 62.69 & 0.00 & 62.63 & 135.14 & 62.60 & 96.77 & 62.69 \\
\hline PSI19 & 14.36 & 0.00 & 14.36 & 0.00 & 14.36 & 0.00 & 14.36 & 0.00 & 14.36 & 12.58 & 14.35 & 20.94 & 14.36 \\
\hline PSI20 & 4.06 & 0.00 & 4.06 & 0.00 & 4.06 & 0.00 & 4.06 & 0.00 & 4.04 & 17.96 & 4.05 & 4.75 & 4.06 \\
\hline
\end{tabular}


Table 20: Unadjusted Rates Stratified by Comorbidities (continued)

\begin{tabular}{|c|c|c|c|c|c|c|c|c|c|c|c|c|c|c|c|}
\hline \multirow{2}{*}{$\begin{array}{l}\text { Comorbidity } \\
\text { PSI }\end{array}$} & \multicolumn{2}{|c|}{23} & \multicolumn{2}{|c|}{25} & \multicolumn{2}{|c|}{26} & \multicolumn{2}{|c|}{27} & \multicolumn{2}{|c|}{28} & \multicolumn{2}{|c|}{29} & \multicolumn{2}{|c|}{30} & \multirow[b]{2}{*}{ Total } \\
\hline & 0 & 1 & 0 & 1 & 0 & 1 & 0 & 1 & 0 & 1 & 0 & 1 & 0 & 1 & \\
\hline All & 16.28 & 61.95 & 16.31 & 50.39 & 16.32 & 36.94 & 16.37 & 22.50 & 16.45 & 14.33 & 16.42 & 24.99 & 16.35 & 29.09 & 16.44 \\
\hline General & 2.62 & 5.54 & 2.61 & 5.48 & 2.62 & 3.59 & 2.64 & 1.53 & 2.63 & 1.74 & 2.63 & 1.97 & 2.63 & 2.39 & 2.63 \\
\hline Medical & 11.59 & 54.86 & 11.72 & 28.81 & 11.66 & 29.65 & 11.67 & 20.18 & 11.83 & 10.29 & 11.78 & 20.97 & 11.67 & 24.65 & 11.81 \\
\hline Post-op & 22.12 & 99.46 & 21.97 & 99.40 & 22.10 & 63.35 & 22.07 & 48.79 & 22.21 & 32.00 & 22.20 & 53.23 & 22.11 & 72.09 & 22.24 \\
\hline Obstetric & 15.58 & 85.32 & 15.65 & 61.86 & 15.68 & 24.73 & 15.69 & 6.54 & 15.70 & 5.76 & 15.69 & 9.17 & 15.69 & 12.84 & 15.69 \\
\hline PSI1 & 0.01 & 0.45 & 0.01 & 0.00 & 0.01 & 0.18 & 0.01 & 0.22 & 0.01 & 0.21 & 0.01 & 0.00 & 0.01 & 0.00 & 0.01 \\
\hline PSI2 & 1.19 & 7.35 & 1.20 & 3.86 & 1.20 & 3.95 & 1.20 & 1.59 & 1.21 & 1.24 & 1.21 & 2.62 & 1.20 & 2.64 & 1.21 \\
\hline PSI3 & 17.02 & 63.20 & 17.41 & 25.89 & 17.23 & 33.15 & 17.53 & 15.69 & 17.62 & 6.49 & 17.48 & 18.34 & 17.23 & 30.28 & 17.49 \\
\hline PSI4 & 102.96 & 166.51 & 103.71 & 99.49 & 104.10 & 78.11 & 102.10 & 152.50 & 103.82 & 84.02 & 103.67 & 100.94 & 103.95 & 84.06 & 103.65 \\
\hline PSI5 & 0.08 & 0.05 & 0.08 & 0.10 & 0.08 & 0.07 & 0.08 & 0.04 & 0.08 & 0.00 & 0.08 & 0.07 & 0.08 & 0.05 & 0.08 \\
\hline PSI6 & 0.36 & 1.65 & 0.37 & 0.41 & 0.37 & 0.62 & 0.37 & 0.38 & 0.37 & 0.56 & 0.37 & 0.51 & 0.37 & 0.54 & 0.37 \\
\hline PSI7 & 4.93 & 7.83 & 4.91 & 9.43 & 4.92 & 7.36 & 4.91 & 6.67 & 4.95 & 4.61 & 4.95 & 4.12 & 4.93 & 6.12 & 4.94 \\
\hline PSI8 & 0.45 & 7.50 & 0.45 & 2.82 & 0.45 & 4.50 & 0.45 & 2.31 & 0.46 & 0.46 & 0.46 & 2.80 & 0.45 & 6.71 & 0.46 \\
\hline PSI9 & 18.70 & 67.36 & 18.54 & 86.53 & 18.69 & 45.93 & 18.64 & 40.65 & 18.76 & 25.84 & 18.75 & 40.44 & 18.69 & 50.61 & 18.78 \\
\hline PSI10 & 1.74 & 22.06 & 1.80 & 2.79 & 1.78 & 7.45 & 1.77 & 9.06 & 1.81 & 0.00 & 1.81 & 0.00 & 1.77 & 8.10 & 1.80 \\
\hline PSI11 & 1.39 & 12.45 & 1.39 & 9.87 & 1.39 & 8.20 & 1.35 & 16.24 & 1.41 & 4.03 & 1.38 & 21.28 & 1.39 & 5.93 & 1.42 \\
\hline PSI12 & 3.15 & 26.19 & 3.15 & 14.51 & 3.15 & 14.66 & 3.17 & 6.50 & 3.18 & 6.21 & 3.18 & 12.97 & 3.15 & 18.71 & 3.19 \\
\hline PSI13 & 12.48 & 77.67 & 12.64 & 37.88 & 12.65 & 27.91 & 12.46 & 43.86 & 12.86 & 0.00 & 12.72 & 28.85 & 12.88 & 6.85 & 12.80 \\
\hline PSI14 & 4.62 & 26.51 & 4.67 & 7.18 & 4.64 & 10.91 & 4.68 & 6.37 & 4.69 & 5.13 & 4.69 & 6.67 & 4.63 & 19.78 & 4.70 \\
\hline PSI15 & 2.22 & 3.45 & 2.22 & 4.58 & 2.23 & 2.54 & 2.25 & 1.01 & 2.24 & 1.13 & 2.23 & 1.20 & 2.24 & 1.51 & 2.23 \\
\hline PSI16 & 0.09 & 0.00 & 0.09 & 0.06 & 0.08 & 0.11 & 0.09 & 0.00 & 0.08 & 1.23 & 0.09 & 0.00 & 0.08 & 1.01 & 0.09 \\
\hline PSI17 & 14.77 & 85.91 & 14.99 & . & 14.99 & 0.00 & 14.99 & . & 14.99 & 0.00 & 14.99 & . & 14.99 & . & 14.99 \\
\hline PSI18 & 62.67 & 500.00 & 62.41 & 164.06 & 62.58 & 108.11 & 62.71 & 0.00 & 62.78 & 12.05 & 62.72 & 0.00 & 62.72 & 38.46 & 62.69 \\
\hline PSI19 & 14.36 & 0.00 & 14.30 & 65.79 & 14.34 & 27.87 & 14.37 & 8.97 & 14.39 & 5.94 & 14.37 & 7.30 & 14.36 & 15.48 & 14.36 \\
\hline PSI20 & 4.06 & 0.00 & 4.05 & 7.46 & 4.05 & 5.24 & 4.06 & 0.00 & 4.06 & 3.39 & 4.05 & 15.87 & 4.06 & 4.03 & 4.06 \\
\hline
\end{tabular}




\section{Appendix D}

Table 21: General

\begin{tabular}{|c|c|c|c|c|c|c|}
\hline Variables & Mean & $\begin{array}{l}\text { Std. } \\
\text { Dev. }\end{array}$ & M1 & M2 & M3 & M4 \\
\hline General & 0.003 & 0.051 & & & & \\
\hline sex (male) & 0.466 & 0.499 & $-0.352 * * *$ & $-0.352 * * *$ & $-0.103 * *$ & $-0.083^{*}$ \\
\hline agecat1 $(<30)$ & 0.117 & 0.322 & $-0.875 * * *$ & $-0.861 * * *$ & $-0.728 * * *$ & $-0.656 * * *$ \\
\hline agecat2 (30-39) & 0.117 & 0.322 & $-0.214 * * *$ & $-0.215 * * *$ & $-0.151 * * *$ & $-0.092^{*}$ \\
\hline agecat4 (40-49) & 0.139 & 0.346 & $0.0972 * *$ & $0.010 * *$ & -0.059 & -0.025 \\
\hline agecat5 (60-69) & 0.160 & 0.366 & $0.101 * *$ & $0.095^{* *}$ & $0.202 * * *$ & $0.165^{* * *}$ \\
\hline agecat6 (70-79) & 0.186 & 0.389 & $0.154 * * *$ & $0.130 * * *$ & $0.315^{* * *}$ & $0.250 * * *$ \\
\hline agecat7 (80+) & 0.157 & 0.364 & $-0.190 * * *$ & $-0.226 * * *$ & 0.069 & -0.006 \\
\hline agesexcat1 & 0.046 & 0.209 & $0.547 * * *$ & $0.547 * * *$ & $0.491 * * *$ & $0.477 * * *$ \\
\hline agesexcat2 & 0.042 & 0.201 & $-0.138 *$ & -0.130 & $-0.177^{* *}$ & $-0.185^{* *}$ \\
\hline agesexcat4 & 0.071 & 0.257 & $-0.237 * * *$ & $-0.235 * * *$ & -0.088 & -0.087 \\
\hline agesexcat5 & 0.086 & 0.280 & $0.221 * * *$ & $0.219 * * *$ & $0.112^{*}$ & 0.102 \\
\hline agesexcat6 & 0.098 & 0.297 & $0.293 * * *$ & $0.291 * * *$ & $0.118^{*}$ & 0.094 \\
\hline agesexcat7 & 0.065 & 0.246 & $0.331 * * *$ & $0.331 * * *$ & 0.0878 & 0.061 \\
\hline Maori & 0.136 & 0.342 & & $-0.148 * * *$ & $-0.107 * * *$ & $-0.111 * * *$ \\
\hline Pacific & 0.049 & 0.216 & & $-0.174 * * *$ & $-0.177 * * *$ & $-0.203 * * *$ \\
\hline Asian & 0.036 & 0.187 & & 0.044 & 0.002 & 0.020 \\
\hline Other & 0.120 & 0.325 & & 0.002 & -0.009 & 0.000 \\
\hline NZDep (lower quintile) & 0.135 & 0.342 & & -0.045 & -0.019 & -0.014 \\
\hline NZDep (second quintile) & 0.160 & 0.367 & & 0.031 & 0.034 & 0.039 \\
\hline NZDep (fourth quintile) & 0.245 & 0.430 & & -0.001 & -0.005 & -0.005 \\
\hline NZDep (upper quintile) & 0.258 & 0.438 & & $-0.090 * * *$ & $-0.091 * * *$ & $-0.087 * * *$ \\
\hline MDC1 Nervous system & 0.063 & 0.243 & & & $-0.536 * * *$ & $-0.441 * * *$ \\
\hline MDC2 Eye & 0.031 & 0.172 & & & $0.605^{* * *}$ & $0.836 * * *$ \\
\hline $\begin{array}{l}\text { MDC3 Ear, nose, mouth and } \\
\text { throat }\end{array}$ & 0.031 & 0.173 & & & $0.130 *$ & $0.285^{* * *}$ \\
\hline MDC4 Respiratory system & 0.074 & 0.261 & & & $0.544 * * *$ & $0.569 * * *$ \\
\hline MDC6 Digestive system & 0.122 & 0.328 & & & $1.110 * * *$ & $1.238^{* * *}$ \\
\hline $\begin{array}{l}\text { MDC7 Hepatobiliary system } \\
\text { and pancreas }\end{array}$ & 0.025 & 0.155 & & & $1.746^{* * *}$ & $1.845^{* * *}$ \\
\hline $\begin{array}{l}\text { MDC8 Musculoskeletal } \\
\text { system }\end{array}$ & 0.102 & 0.303 & & & $0.123^{* * *}$ & $0.274^{* * *}$ \\
\hline $\begin{array}{l}\text { MDC9 Skin, subcutaneous } \\
\text { tissue and breast }\end{array}$ & 0.062 & 0.241 & & & $-0.912 * * *$ & $-0.746 * * *$ \\
\hline $\begin{array}{l}\text { MDC10 Endocrine, } \\
\text { nutritional and metabolic }\end{array}$ & 0.015 & 0.120 & & & $0.268 * * *$ & $0.298 * * *$ \\
\hline $\begin{array}{l}\text { MDC11 Kidney and urinary } \\
\text { tract }\end{array}$ & 0.076 & 0.265 & & & $0.226 * * *$ & $0.539 * * *$ \\
\hline $\begin{array}{l}\text { MDC12 Male reproductive } \\
\text { system }\end{array}$ & 0.009 & 0.096 & & & $0.772 * * *$ & $0.919 * * *$ \\
\hline
\end{tabular}




\begin{tabular}{|c|c|c|c|c|}
\hline $\begin{array}{l}\text { MDC13 Female } \\
\text { reproductive system }\end{array}$ & 0.039 & 0.193 & $1.852 * * *$ & $2.003 * * *$ \\
\hline MDC14 Pregnancy & 0.048 & 0.215 & $-3.612 * * *$ & $-3.461 * * *$ \\
\hline $\begin{array}{l}\text { MDC16 Blood and } \\
\text { immunological disorders }\end{array}$ & 0.017 & 0.128 & $-0.703 * * *$ & $-0.539 * * *$ \\
\hline MDC17 Neoplastic disorders & 0.028 & 0.164 & $-0.613 * * *$ & $-0.497 * * *$ \\
\hline $\begin{array}{l}\text { MDC18 Infectious and } \\
\text { parasitic diseases }\end{array}$ & 0.015 & 0.123 & $0.315^{* * *}$ & $0.413 * * *$ \\
\hline $\begin{array}{l}\text { MDC19 Mental diseases and } \\
\text { disorders }\end{array}$ & 0.016 & 0.124 & $-2.479 * * *$ & $-2.346 * * *$ \\
\hline MDC20 Alcohol/drug & 0.003 & 0.051 & $-2.166 * * *$ & $-1.930 * * *$ \\
\hline MDC21 Injuries, poisoning & 0.029 & 0.167 & $0.315^{* * *}$ & $0.483 * * *$ \\
\hline MDC22 Burns & 0.001 & 0.034 & 0.296 & 0.454 \\
\hline MDC23 Other Factors & 0.052 & 0.223 & $0.240 * * *$ & $0.273 * * *$ \\
\hline Congestive heart failure & 0.035 & 0.183 & & $0.222 * * *$ \\
\hline Valvular disease & 0.017 & 0.128 & & $0.365^{* * *}$ \\
\hline $\begin{array}{l}\text { Pulmonary circulation } \\
\text { disorders }\end{array}$ & 0.005 & 0.070 & & $0.468 * * *$ \\
\hline $\begin{array}{l}\text { Peripheral vascular } \\
\text { disorders }\end{array}$ & 0.017 & 0.130 & & $0.728 * * *$ \\
\hline Hypertension combined & 0.128 & 0.335 & & $0.401 * * *$ \\
\hline Paralysis & 0.025 & 0.156 & & 0.0401 \\
\hline $\begin{array}{l}\text { Other neurological } \\
\text { disorders }\end{array}$ & 0.020 & 0.140 & & $-0.160 * *$ \\
\hline Chronic pulmonary disease & 0.029 & 0.167 & & $0.549 * * *$ \\
\hline $\begin{array}{l}\text { Diabetes uncomplicated } \\
\text { variation }\end{array}$ & 0.044 & 0.204 & & $-0.257 * * *$ \\
\hline Diabetes complicated & 0.048 & 0.214 & & $-0.280 * * *$ \\
\hline Hypothyroidism & 0.004 & 0.060 & & 0.072 \\
\hline Renal failure & 0.072 & 0.259 & & $-0.357 * * *$ \\
\hline Liver disease & 0.008 & 0.087 & & $0.226 * * *$ \\
\hline $\begin{array}{l}\text { Peptic ulcer disease } \\
\text { excluding bleeding }\end{array}$ & 0.001 & 0.034 & & $0.443 * * *$ \\
\hline AIDS & 0.000 & 0.019 & & -0.184 \\
\hline Lymphoma & 0.007 & 0.086 & & $-0.571 * * *$ \\
\hline Metastatic cancer & 0.028 & 0.166 & & $0.511 * * *$ \\
\hline $\begin{array}{l}\text { Solid tumour without } \\
\text { metastasis variation }\end{array}$ & 0.018 & 0.132 & & $0.559 * * *$ \\
\hline $\begin{array}{l}\text { Rheumatoid arthritis / } \\
\text { collagen vascular diseases }\end{array}$ & 0.007 & 0.086 & & -0.122 \\
\hline Obesity & 0.017 & 0.130 & & $0.371 * * *$ \\
\hline Weight loss & 0.004 & 0.063 & & $0.485^{* * *}$ \\
\hline Blood loss anaemia & 0.004 & 0.066 & & 0.119 \\
\hline Deficiency anaemias & 0.007 & 0.081 & & 0.150 \\
\hline Alcohol abuse & 0.014 & 0.119 & & $-0.467 * * *$ \\
\hline Drug abuse & 0.006 & 0.077 & & $0.397 * * *$ \\
\hline Psychoses & 0.003 & 0.055 & & 0.030 \\
\hline Depression & 0.009 & 0.093 & & 0.117 \\
\hline
\end{tabular}




\begin{tabular}{lllll} 
Constant & $-5.793^{* * *}$ & $-5.736^{* * *}$ & $-6.373^{* * *}$ & $-6.596 * * *$ \\
Observations & $4,768,505$ & $4,768,505$ & $4,768,505$ & $4,768,505$ \\
\hline Sensitivity & 0.596 & 0.607 & 0.634 & 0.657 \\
Specificity & 0.521 & 0.510 & 0.680 & 0.683 \\
C-stat (Area under ROC) & 0.577 & 0.581 & 0.718 & 0.733 \\
LR test M1 & & $77^{* * *}$ & $7306^{* * *}$ & $8434^{* * *}$ \\
LR test M2 & & & $7230^{* * *}$ & $8358^{* * *}$ \\
LR test M3 & & & & $1128^{* * *}$ \\
\hline$* * *$
\end{tabular}


Table 22: Medical

\begin{tabular}{|c|c|c|c|c|c|c|}
\hline Variables & Mean & $\begin{array}{l}\text { Std. } \\
\text { Dev. }\end{array}$ & M1 & M2 & M3 & M4 \\
\hline Medical & 0.012 & 0.108 & & & & \\
\hline sex (male) & 0.450 & 0.497 & $0.094^{* * *}$ & $0.104^{* * *}$ & $0.077^{* *}$ & 0.045 \\
\hline agecat1 $(<30)$ & 0.123 & 0.328 & $-1.932 * * *$ & $-1.990 * * *$ & $-1.395 * * *$ & $-1.109 * * *$ \\
\hline agecat2 (30-39) & 0.115 & 0.319 & $-1.445^{* * *}$ & $-1.455^{* * *}$ & $-0.968 * * *$ & $-0.759 * * *$ \\
\hline agecat4 (40-49) & 0.128 & 0.334 & $-0.602 * * *$ & $-0.618 * * *$ & $-0.512 * * *$ & $-0.405 * * *$ \\
\hline agecat5 (60-69) & 0.151 & 0.358 & $0.308 * * *$ & $0.328 * * *$ & $0.290 * * *$ & $0.214^{* * *}$ \\
\hline agecat6 (70-79) & 0.190 & 0.392 & $0.562 * * *$ & $0.616^{* * *}$ & $0.511 * * *$ & $0.410 * * *$ \\
\hline agecat7 (80+) & 0.177 & 0.382 & $0.735 * * *$ & $0.816^{* * *}$ & $0.618 * * *$ & $0.524 * * *$ \\
\hline agesexcat1 & 0.041 & 0.199 & $0.889 * * *$ & $0.895^{* * *}$ & $0.356 * * *$ & $0.329 * * *$ \\
\hline agesexcat2 & 0.040 & 0.196 & $0.622 * * *$ & $0.598 * * *$ & $0.154^{* *}$ & $0.137 * *$ \\
\hline agesexcat4 & 0.066 & 0.249 & $0.222 * * *$ & $0.222 * * *$ & $0.139 * * *$ & $0.136^{* * *}$ \\
\hline agesexcat5 & 0.081 & 0.272 & $0.107 * * *$ & $0.106 * * *$ & $0.117 * * *$ & $0.103 * *$ \\
\hline agesexcat6 & 0.097 & 0.296 & 0.002 & -0.003 & 0.037 & -0.020 \\
\hline agesexcat7 & 0.070 & 0.256 & $-0.091 * *$ & $-0.010 * * *$ & -0.034 & $-0.136 * * *$ \\
\hline Maori & 0.121 & 0.326 & & $0.406 * * *$ & $0.369 * * *$ & $0.177^{* * *}$ \\
\hline Pacific & 0.051 & 0.220 & & $0.234 * * *$ & $0.204 * * *$ & 0.028 \\
\hline Asian & 0.035 & 0.184 & & $-0.186 * * *$ & $-0.188 * * *$ & $-0.206 * * *$ \\
\hline Other & 0.122 & 0.328 & & $-0.045 * * *$ & $-0.037 * *$ & -0.025 \\
\hline NZDep (lower quintile) & 0.136 & 0.343 & & $-0.034^{*}$ & $-0.044 * *$ & -0.029 \\
\hline NZDep (second quintile) & 0.162 & 0.368 & & $-0.058 * * *$ & $-0.061 * * *$ & $-0.047 * * *$ \\
\hline NZDep (fourth quintile) & 0.246 & 0.430 & & -0.006 & -0.001 & -0.009 \\
\hline NZDep (upper quintile) & 0.253 & 0.435 & & $0.047 * * *$ & $0.058 * * *$ & $0.051 * * *$ \\
\hline MDC1 Nervous system & 0.061 & 0.239 & & & $0.391 * * *$ & $0.497 * * *$ \\
\hline MDC2 Eye & 0.005 & 0.073 & & & $-1.875^{* * *}$ & $-1.585 * * *$ \\
\hline $\begin{array}{l}\text { MDC3 Ear, nose, mouth and } \\
\text { throat }\end{array}$ & 0.021 & 0.142 & & & $-0.686 * * *$ & $-0.501 * * *$ \\
\hline MDC4 Respiratory system & 0.082 & 0.274 & & & $0.308 * * *$ & $0.167 * * *$ \\
\hline MDC6 Digestive system & 0.144 & 0.351 & & & $-0.010 * * *$ & $-0.061 * * *$ \\
\hline $\begin{array}{l}\text { MDC7 Hepatobiliary system } \\
\text { and pancreas }\end{array}$ & 0.030 & 0.169 & & & $0.253^{* * *}$ & $0.118^{* * *}$ \\
\hline $\begin{array}{l}\text { MDC8 Musculoskeletal } \\
\text { system }\end{array}$ & 0.107 & 0.310 & & & $0.115 * * *$ & $0.260 * * *$ \\
\hline $\begin{array}{l}\text { MDC9 Skin, subcutaneous } \\
\text { tissue and breast }\end{array}$ & 0.064 & 0.244 & & & $-1.033 * * *$ & $-0.744 * * *$ \\
\hline $\begin{array}{l}\text { MDC10 Endocrine, } \\
\text { nutritional and metabolic }\end{array}$ & 0.015 & 0.122 & & & $0.826 * * *$ & $0.491^{* * *}$ \\
\hline $\begin{array}{l}\text { MDC11 Kidney and urinary } \\
\text { tract }\end{array}$ & 0.037 & 0.188 & & & $0.349 * * *$ & $0.280 * * *$ \\
\hline $\begin{array}{l}\text { MDC12 Male reproductive } \\
\text { system }\end{array}$ & 0.005 & 0.071 & & & $-0.496 * * *$ & $-0.422 * * *$ \\
\hline $\begin{array}{l}\text { MDC13 Female reproductive } \\
\text { system }\end{array}$ & 0.027 & 0.163 & & & $-0.869 * * *$ & $-0.769 * * *$ \\
\hline MDC14 Pregnancy & 0.064 & 0.245 & & & $-3.331 * * *$ & $-3.206 * * *$ \\
\hline MDC16 Blood and & 0.013 & 0.113 & & & $-0.474 * * *$ & $-0.388 * * *$ \\
\hline
\end{tabular}


immunological disorders

MDC17 Neoplastic disorders
MDC18 Infectious and
parasitic diseases
MDC19 Mental diseases and
disorders
MDC20 Alcohol/drug

$0.011 \quad 0.103$

$0.017 \quad 0.129$

$0.023 \quad 0.150$

$0.003 \quad 0.058$

MDC21 Injuries, poisoning

$0.027 \quad 0.161$

MDC22 Burns

$0.001 \quad 0.032$

MDC23 Other Factors

$0.060 \quad 0.237$

$0.044 \quad 0.205$

$0.021 \quad 0.144$

$0.007 \quad 0.081$

$0.020 \quad 0.139$

$0.150 \quad 0.357$

$0.029 \quad 0.168$

$0.025 \quad 0.156$

$0.036 \quad 0.185$

$0.051 \quad 0.220$

$0.043 \quad 0.202$

$0.005 \quad 0.068$

$0.031 \quad 0.172$

$0.008 \quad 0.088$

$0.002 \quad 0.039$

$0.000 \quad 0.013$

$0.003 \quad 0.050$

$0.017 \quad 0.128$

$0.009 \quad 0.094$

$0.008 \quad 0.090$

$0.019 \quad 0.137$

$0.005 \quad 0.071$

$0.005 \quad 0.072$

$0.009 \quad 0.092$

$0.017 \quad 0.128$

$0.007 \quad 0.086$

$0.004 \quad 0.061$

$0.011 \quad 0.106$

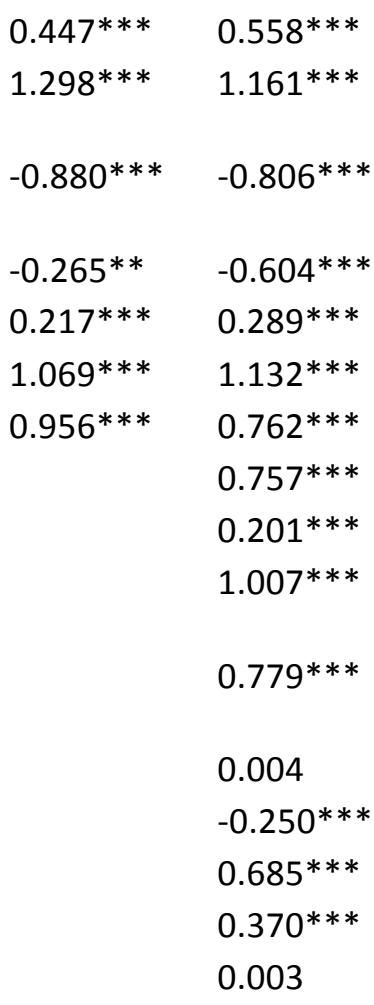

$0.403^{* * *}$

$-0.030$

$0.582^{* * *}$

$1.279 * * *$

$0.456 * * *$

$0.917 * * *$

$1.064 * * *$

$1.437^{* * *}$

$0.730 * * *$

$0.529 * * *$

$0.423^{* * *}$

$1.081^{* * *}$

$0.613 * * *$

$0.351 * * *$

$0.327 * * *$

$0.471 * * *$

$0.498 * * *$

$0.386 * * *$

$-4.625 * * * \quad-4.703 * * * \quad-4.803 * * * \quad-5.121 * * *$

$3,201,407 \quad 3,201,407 \quad 3,201,407 \quad 3,201,407$

$\begin{array}{llll}0.774 & 0.790 & 0.777 & 0.700\end{array}$

0.485

0.476

0.547

0.729

Specificity 


\begin{tabular}{lllll} 
C-stat $($ Area under ROC) & 0.663 & 0.670 & 0.719 & 0.789 \\
LR test M1 & & $772^{* * *}$ & $10376^{* * *}$ & $29069^{* * *}$ \\
LR test M2 & & & $9586^{* * *}$ & $28297^{* * *}$ \\
LR test M3 & & & & $18711^{* * *}$ \\
\hline
\end{tabular}

*** $p<0.01,{ }^{* *} p<0.05, * p<0.1$ 
Table 23: Surgical

\begin{tabular}{|c|c|c|c|c|c|c|}
\hline Variables & Mean & $\begin{array}{l}\text { Std. } \\
\text { Dev. }\end{array}$ & M1 & M2 & M3 & M4 \\
\hline Surgical & 0.022 & 0.147 & & & & \\
\hline sex (male) & 0.483 & 0.500 & $0.231 * * *$ & $0.238 * * *$ & -0.012 & 0.024 \\
\hline agecat1 $(<30)$ & 0.123 & 0.328 & $-0.758 * * *$ & $-0.785 * * *$ & $-0.726 * * *$ & $-0.570 * * *$ \\
\hline agecat2 (30-39) & 0.122 & 0.327 & $-0.422 * * *$ & $-0.448 * * *$ & $-0.389 * * *$ & $-0.268 * * *$ \\
\hline agecat4 (40-49) & 0.147 & 0.354 & $-0.141 * * *$ & $-0.156 * * *$ & $-0.119 * * *$ & $-0.061^{*}$ \\
\hline agecat5 (60-69) & 0.163 & 0.370 & $0.257 * * *$ & $0.273 * * *$ & $0.267^{* * *}$ & $0.194 * * *$ \\
\hline agecat6 (70-79) & 0.180 & 0.384 & $0.400 * * *$ & $0.441^{* * *}$ & $0.495^{* * *}$ & $0.385^{* * *}$ \\
\hline agecat7 (80+) & 0.120 & 0.324 & $0.344 * * *$ & $0.400 * * *$ & $0.588 * * *$ & $0.453 * * *$ \\
\hline agesexcat1 & 0.065 & 0.246 & $0.096^{*}$ & 0.0784 & $0.239 * * *$ & $0.229 * * *$ \\
\hline agesexcat2 & 0.051 & 0.220 & $-0.159 * * *$ & $-0.163 * * *$ & -0.059 & -0.068 \\
\hline agesexcat4 & 0.071 & 0.257 & $-0.148 * * *$ & $-0.149 * * *$ & $-0.106^{* *}$ & $-0.109 * *$ \\
\hline agesexcat5 & 0.086 & 0.281 & 0.001 & 0.000 & 0.003 & 0.016 \\
\hline agesexcat6 & 0.096 & 0.294 & -0.022 & -0.025 & 0.011 & 0.004 \\
\hline agesexcat7 & 0.051 & 0.221 & -0.028 & -0.034 & 0.034 & 0.033 \\
\hline Maori & 0.114 & 0.318 & & $0.286 * * *$ & $0.262 * * *$ & $0.146 * * *$ \\
\hline Pacific & 0.046 & 0.210 & & $0.377^{* * *}$ & $0.438 * * *$ & $0.294^{* * *}$ \\
\hline Asian & 0.034 & 0.181 & & $0.107^{* * *}$ & $0.157^{* * *}$ & $0.144^{* * *}$ \\
\hline Other & 0.123 & 0.328 & & -0.020 & $-0.036 * *$ & -0.011 \\
\hline NZDep (lower quintile) & 0.137 & 0.344 & & 0.027 & -0.004 & 0.005 \\
\hline NZDep (second quintile) & 0.165 & 0.371 & & 0.0282 & 0.010 & 0.023 \\
\hline NZDep (fourth quintile) & 0.247 & 0.432 & & $-0.029 *$ & -0.015 & -0.010 \\
\hline NZDep (upper quintile) & 0.244 & 0.429 & & $-0.055^{* * *}$ & $-0.031 *$ & $-0.044 * *$ \\
\hline MDC1 Nervous system & 0.038 & 0.192 & & & $0.534^{* * *}$ & $0.458 * * *$ \\
\hline MDC2 Eye & 0.089 & 0.284 & & & $-3.292 * * *$ & $-3.064 * * *$ \\
\hline $\begin{array}{l}\text { MDC3 Ear, nose, mouth and } \\
\text { throat }\end{array}$ & 0.054 & 0.227 & & & $-0.123 * * *$ & -0.035 \\
\hline MDC4 Respiratory system & 0.022 & 0.147 & & & $0.184^{* * *}$ & $-0.190 * * *$ \\
\hline MDC5 Circulatory system & 0.081 & 0.273 & & & $1.254^{* * *}$ & $0.980 * * *$ \\
\hline MDC6 Digestive system & 0.108 & 0.310 & & & $0.411 * * *$ & $0.285 * * *$ \\
\hline $\begin{array}{l}\text { MDC7 Hepatobiliary system } \\
\text { and pancreas }\end{array}$ & 0.029 & 0.167 & & & $0.471 * * *$ & $0.420 * * *$ \\
\hline $\begin{array}{l}\text { MDC9 Skin, subcutaneous } \\
\text { tissue and breast }\end{array}$ & 0.133 & 0.339 & & & $-0.481 * * *$ & $-0.348 * * *$ \\
\hline $\begin{array}{l}\text { MDC10 Endocrine, } \\
\text { nutritional and metabolic }\end{array}$ & 0.012 & 0.109 & & & $0.536 * * *$ & $0.298 * * *$ \\
\hline $\begin{array}{l}\text { MDC11 Kidney and urinary } \\
\text { tract }\end{array}$ & 0.039 & 0.193 & & & $0.530 * * *$ & $0.468 * * *$ \\
\hline $\begin{array}{l}\text { MDC12 Male reproductive } \\
\text { system }\end{array}$ & 0.020 & 0.139 & & & $0.616^{* * *}$ & $0.711^{* * *}$ \\
\hline $\begin{array}{l}\text { MDC13 Female } \\
\text { reproductive system }\end{array}$ & 0.106 & 0.307 & & & -0.027 & $0.051^{*}$ \\
\hline MDC14 Pregnancy & 0.003 & 0.055 & & & $0.789 * * *$ & $0.668 * * *$ \\
\hline MDC17 Neoplastic disorders & 0.009 & 0.095 & & & $0.718^{* * *}$ & $0.434 * * *$ \\
\hline MDC18 Infectious and & 0.008 & 0.087 & & & $1.639 * * *$ & $1.487 * * *$ \\
\hline
\end{tabular}




\begin{tabular}{|c|c|c|c|c|c|c|}
\hline $\begin{array}{l}\text { MDC19 Mental diseases and } \\
\text { disorders }\end{array}$ & 0.004 & 0.060 & & & $-2.066 * * *$ & $-1.941 * * *$ \\
\hline MDC21 Injuries, poisoning & 0.000 & 0.005 & & & $0.890 * * *$ & $0.878 * * *$ \\
\hline MDC22 Burns & 0.030 & 0.170 & & & $0.442 * * *$ & $0.387 * * *$ \\
\hline MDC23 Other Factors & 0.002 & 0.042 & & & $0.159 * * *$ & -0.082 \\
\hline Congestive heart failure & 0.010 & 0.100 & & & & $0.183^{* * *}$ \\
\hline Valvular disease & 0.016 & 0.124 & & & & $0.497 * * *$ \\
\hline $\begin{array}{l}\text { Pulmonary circulation } \\
\text { disorders }\end{array}$ & 0.010 & 0.100 & & & & $3.563 * * *$ \\
\hline $\begin{array}{l}\text { Peripheral vascular } \\
\text { disorders }\end{array}$ & 0.003 & 0.054 & & & & $0.429 * * *$ \\
\hline Hypertension combined & 0.017 & 0.130 & & & & $0.474 * * *$ \\
\hline Paralysis & 0.103 & 0.304 & & & & $0.600 * * *$ \\
\hline $\begin{array}{l}\text { Other neurological } \\
\text { disorders }\end{array}$ & 0.009 & 0.096 & & & & $0.234 * * *$ \\
\hline Chronic pulmonary disease & 0.007 & 0.083 & & & & $0.111^{* * *}$ \\
\hline $\begin{array}{l}\text { Diabetes uncomplicated } \\
\text { variation }\end{array}$ & 0.015 & 0.121 & & & & $-0.105 * * *$ \\
\hline Diabetes complicated & 0.036 & 0.186 & & & & $-0.209 * * *$ \\
\hline Hypothyroidism & 0.035 & 0.184 & & & & $0.309 * * *$ \\
\hline Renal failure & 0.002 & 0.043 & & & & $0.297 * * *$ \\
\hline Liver disease & 0.028 & 0.164 & & & & $0.870 * * *$ \\
\hline $\begin{array}{l}\text { Peptic ulcer disease } \\
\text { excluding bleeding }\end{array}$ & 0.004 & 0.065 & & & & $0.584 * * *$ \\
\hline AIDS & 0.001 & 0.024 & & & & -0.675 \\
\hline Lymphoma & 0.000 & 0.014 & & & & $0.333 * * *$ \\
\hline Metastatic cancer & 0.002 & 0.039 & & & & $0.858 * * *$ \\
\hline $\begin{array}{l}\text { Solid tumour without } \\
\text { metastasis variation }\end{array}$ & 0.024 & 0.153 & & & & $0.578 * * *$ \\
\hline $\begin{array}{l}\text { Rheumatoid arthritis / } \\
\text { collagen vascular diseases }\end{array}$ & 0.013 & 0.112 & & & & $0.230 * * *$ \\
\hline Obesity & 0.005 & 0.069 & & & & $0.414 * * *$ \\
\hline Weight loss & 0.014 & 0.119 & & & & $0.902 * * *$ \\
\hline Blood loss anaemia & 0.002 & 0.039 & & & & $1.162 * * *$ \\
\hline Deficiency anaemias & 0.004 & 0.059 & & & & $0.376 * * *$ \\
\hline Alcohol abuse & 0.003 & 0.057 & & & & $0.533 * * *$ \\
\hline Drug abuse & 0.006 & 0.080 & & & & $0.416 * * *$ \\
\hline Psychoses & 0.003 & 0.054 & & & & $0.619 * * *$ \\
\hline Depression & 0.001 & 0.037 & & & & $0.705^{* * *}$ \\
\hline Constant & 0.003 & 0.051 & $-3.942 * * *$ & $-3.997 * * *$ & $-4.198 * * *$ & $-4.404 * * *$ \\
\hline Observations & & & $1,424,249$ & $1,424,249$ & $1,424,249$ & $1,424,249$ \\
\hline Sensitivity & & & 0.692 & 0.692 & 0.688 & 0.671 \\
\hline Specificity & & & 0.469 & 0.474 & 0.639 & 0.726 \\
\hline C-stat (Area under ROC) & & & 0.604 & 0.609 & 0.721 & 0.767 \\
\hline LR test M1 & & & & $357^{* * *}$ & $16051^{* * *}$ & $31385^{* * *}$ \\
\hline LR test M2 & & & & & $15695^{* * *}$ & $31027 * * *$ \\
\hline
\end{tabular}


${ }^{* * *} \mathrm{p}<0.01,{ }^{* *} \mathrm{p}<0.05,{ }^{*} \mathrm{p}<0.1$ 
Table 24: Obstetrics

\begin{tabular}{lllll}
\hline Variables & Mean & Std. Dev. & M1 & M2 \\
\hline Obstetrics & 0.016 & 0.124 & & \\
sex (male) & 0.257 & 0.437 & $0.127^{* * *}$ & $0.104^{* * *}$ \\
agecat1 (<30) & 0.751 & 0.433 & $-0.039^{*}$ & $0.0384^{*}$ \\
agecat3 (40-49) & 0.232 & 0.422 & $-0.754^{* * *}$ & $-0.751^{* * *}$ \\
Maori & 0.257 & 0.437 & & $-0.463^{* * *}$ \\
Pacific & 0.205 & 0.403 & & $0.129^{* * *}$ \\
Asian & 0.109 & 0.311 & & $0.800^{* * *}$ \\
Other & 0.088 & 0.284 & & $0.204^{* * *}$ \\
NZDep (lower quintile) & 0.092 & 0.289 & & -0.016 \\
NZDep (second quintile) & 0.150 & 0.357 & & 0.024 \\
NZDep (fourth quintile) & 0.160 & 0.366 & & -0.031 \\
NZDep (upper quintile) & 0.220 & 0.414 & & $-0.069^{* * *}$ \\
Constant & 0.001 & 0.025 & $-4.134^{* * *}$ & $-4.227^{* * *}$ \\
Observations & & & 980,484 & 980,484 \\
\hline Sensitivity & & & 0.515 & 0.496 \\
Specificity & & & 0.511 & 0.621 \\
C-stat (Area under ROC) & & & 0.518 & 0.590 \\
LR test M1 & & & & $1897^{* * *}$ \\
\hline
\end{tabular}

$* * * p<0.01,{ }^{* *} p<0.05, * p<0.1$ 
Table 25: PSI1

\begin{tabular}{|c|c|c|c|c|c|c|}
\hline Variables & Mean & Std. Dev. & M1 & M2 & M3 & M4 \\
\hline PSI1 & 0.000 & 0.004 & & & & \\
\hline sex (male) & 0.415 & 0.493 & -1.061 & -1.067 & -1.195 & $-1.409 *$ \\
\hline agecat1 (<30) & 0.170 & 0.376 & 0.325 & 0.317 & 0.364 & 0.51 \\
\hline agecat2 (30-39) & 0.171 & 0.377 & 0.782 & 0.751 & 0.686 & 0.774 \\
\hline agecat5 (60-69) & 0.140 & 0.347 & -0.358 & -0.325 & -0.467 & -0.51 \\
\hline agecat6 (70-79) & 0.155 & 0.362 & -0.087 & -0.038 & -0.23 & -0.313 \\
\hline agecat7 (80+) & 0.103 & 0.304 & 0.456 & 0.513 & 0.245 & 0.117 \\
\hline agesexcat2 & 0.044 & 0.205 & 0.334 & 0.359 & 0.666 & 0.712 \\
\hline agesexcat5 & 0.074 & 0.262 & 0.948 & 0.942 & 0.515 & 0.591 \\
\hline agesexcat7 & 0.044 & 0.205 & 1.753 & 1.753 & 2.03 & $2.164 *$ \\
\hline Maori & 0.124 & 0.329 & & 0.632 & 0.679 & 0.517 \\
\hline Other & 0.120 & 0.325 & & 0.423 & 0.407 & 0.444 \\
\hline NZDep (lower quintile) & 0.139 & 0.346 & & 0.803 & 0.783 & 0.801 \\
\hline NZDep (second quintile) & 0.165 & 0.371 & & 0.224 & 0.213 & 0.231 \\
\hline NZDep (fourth quintile) & 0.245 & 0.430 & & 0.218 & 0.234 & 0.224 \\
\hline NZDep (upper quintile) & 0.245 & 0.430 & & -0.242 & -0.223 & -0.28 \\
\hline DRG3 & 0.004 & 0.062 & & & $3.244^{* * *}$ & $3.033^{* * *}$ \\
\hline DRG9 & 0.016 & 0.126 & & & 1.689 & 1.304 \\
\hline DRG15 & 0.018 & 0.133 & & & $2.089 * * *$ & $2.19 * * *$ \\
\hline DRG17 & 0.041 & 0.198 & & & $1.492^{*}$ & 1.339 \\
\hline DRG18 & 0.013 & 0.112 & & & 1.583 & 1.062 \\
\hline DRG29 & 0.002 & 0.044 & & & $4.694 * * *$ & $5.003 * * *$ \\
\hline DRG34 & 0.059 & 0.235 & & & 1.029 & $1.154 *$ \\
\hline DRG35 & 0.011 & 0.106 & & & $2.078 * *$ & $1.796^{*}$ \\
\hline Congestive heart failure & 0.014 & 0.115 & & & & 1.109 \\
\hline Pulmonary circulation disorders & 0.003 & 0.051 & & & & $2.059 *$ \\
\hline Peripheral vascular disorders & 0.015 & 0.120 & & & & 1.068 \\
\hline Hypertension combined & 0.089 & 0.284 & & & & 0.199 \\
\hline Paralysis & 0.008 & 0.090 & & & & 1.49 \\
\hline Chronic pulmonary disease & 0.013 & 0.114 & & & & 0.083 \\
\hline Diabetes uncomplicated variation & 0.031 & 0.174 & & & & 0.339 \\
\hline Diabetes complicated & 0.031 & 0.172 & & & & -0.068 \\
\hline Renal failure & 0.024 & 0.152 & & & & -0.116 \\
\hline Liver disease & 0.004 & 0.061 & & & & 1.203 \\
\hline Obesity & 0.013 & 0.115 & & & & 1.139 \\
\hline Weight loss & 0.001 & 0.037 & & & & $2.536 * *$ \\
\hline Deficiency anaemias & 0.003 & 0.057 & & & & $1.818^{*}$ \\
\hline Alcohol abuse & 0.006 & 0.074 & & & & $2.432 * * *$ \\
\hline Drug abuse & 0.003 & 0.053 & & & & $2.012^{*}$ \\
\hline Constant & & & \multicolumn{4}{|c|}{$-11.25 * * *-11.60 * * *-12.08 * * *-12.34 * * *$} \\
\hline Observations & & & \multicolumn{4}{|c|}{ 1,654,181 1,654,181 1,654,181 1,654,181 } \\
\hline
\end{tabular}




\begin{tabular}{lllll}
\hline Sensitivity & 0.625 & 0.750 & 0.792 & 0.750 \\
Specificity & 0.655 & 0.621 & 0.751 & 0.792 \\
C-stat (Area under ROC) & 0.677 & 0.711 & 0.808 & 0.859 \\
LR test M1 & & 3.8 & $25.39^{* *}$ & $51.23^{* * *}$ \\
LR test M2 & & & $21.58^{* * *}$ & $47.42^{* * *}$ \\
LR test M3 & & & & \\
\hline
\end{tabular}

$* * * p<0.01, * * p<0.05, * p<0.1$ 
Table 26: PSI3

\begin{tabular}{|c|c|c|c|c|c|c|}
\hline Variables & Mean & $\begin{array}{l}\text { Std. } \\
\text { Dev. }\end{array}$ & M1 & M2 & M3 & M4 \\
\hline PSI3 & 0.017 & 0.131 & & & & \\
\hline sex (male) & 0.470 & 0.499 & $-0.165 * * *$ & $-0.163^{* *}$ & $-0.196 * * *$ & $-0.206 * * *$ \\
\hline agecat1 $(<30)$ & 0.050 & 0.219 & $-1.209 * * *$ & $-1.226 * * *$ & $-1.217 * * *$ & $-1.031 * * *$ \\
\hline agecat2 (30-39) & 0.055 & 0.228 & $-1.152 * * *$ & $-1.165^{* * *}$ & $-1.113^{* * *}$ & $-0.979 * * *$ \\
\hline agecat3 (40-49) & 0.080 & 0.272 & $-0.524 * * *$ & $-0.532 * * *$ & $-0.477 * * *$ & $-0.416 * * *$ \\
\hline agecat5 (60-69) & 0.165 & 0.371 & $0.205^{* * *}$ & $0.219 * * *$ & $0.151 * * *$ & $0.138 * *$ \\
\hline agecat6 (70-79) & 0.250 & 0.433 & $0.607^{* * *}$ & $0.645^{* * *}$ & $0.447 * * *$ & $0.461 * * *$ \\
\hline agecat7 (80+) & 0.289 & 0.453 & $1.130 * * *$ & $1.183^{* * *}$ & $0.797 * * *$ & $0.862 * * *$ \\
\hline agesexcat1 & 0.028 & 0.164 & $0.832 * * *$ & $0.822 * * *$ & $0.728 * * *$ & $0.757 * * *$ \\
\hline agesexcat2 & 0.027 & 0.162 & $0.588 * * *$ & $0.580 * * *$ & $0.490 * * *$ & $0.522 * * *$ \\
\hline agesexcat3 & 0.040 & 0.195 & $0.485^{* * *}$ & $0.483 * * *$ & $0.407 * * *$ & $0.433 * * *$ \\
\hline agesexcat5 & 0.087 & 0.281 & $0.190 * *$ & $0.189 * *$ & $0.226 * * *$ & $0.201 * *$ \\
\hline agesexcat6 & 0.124 & 0.329 & $0.253^{* * *}$ & $0.251^{* * *}$ & $0.331 * * *$ & $0.259 * * *$ \\
\hline agesexcat7 & 0.108 & 0.311 & $0.233 * * *$ & $0.231 * * *$ & $0.339 * * *$ & $0.238 * * *$ \\
\hline Maori & 0.104 & 0.306 & & $0.238 * * *$ & $0.257 * * *$ & $0.141 * * *$ \\
\hline Pacific & 0.040 & 0.196 & & 0.060 & $0.079 *$ & -0.054 \\
\hline Asian & 0.024 & 0.155 & & $-0.435^{* * *}$ & $-0.406 * * *$ & $-0.451 * * *$ \\
\hline Other & 0.124 & 0.330 & & $-0.069 * * *$ & $-0.053 * *$ & $-0.056 * *$ \\
\hline NZDep (lower quintile) & 0.134 & 0.340 & & -0.023 & -0.041 & -0.035 \\
\hline NZDep (second quintile) & 0.164 & 0.371 & & $-0.088 * * *$ & $-0.091 * * *$ & $-0.084 * * *$ \\
\hline NZDep (fourth quintile) & 0.251 & 0.434 & & -0.000 & 0.005 & 0.002 \\
\hline NZDep (upper quintile) & 0.242 & 0.428 & & $0.073 * * *$ & $0.082 * * *$ & $0.083 * * *$ \\
\hline DRG1 & 0.006 & 0.079 & & & $-0.888 * * *$ & $-0.675 * * *$ \\
\hline DRG2 & 0.001 & 0.023 & & & 0.206 & 0.397 \\
\hline DRG3 & 0.001 & 0.025 & & & $-1.345^{*}$ & $-1.189 *$ \\
\hline DRG4 & 0.000 & 0.021 & & & 0.511 & 0.551 \\
\hline DRG6 & 0.003 & 0.054 & & & -0.0270 & -0.148 \\
\hline DRG7 & 0.003 & 0.058 & & & $0.644^{* * *}$ & $0.658 * * *$ \\
\hline DRG8 & 0.001 & 0.030 & & & $1.600 * * *$ & $1.720 * * *$ \\
\hline DRG11 & 0.011 & 0.105 & & & $-0.414 * * *$ & $-0.413 * * *$ \\
\hline DRG12 & 0.002 & 0.050 & & & -0.0571 & -0.137 \\
\hline DRG13 & 0.002 & 0.040 & & & 0.163 & 0.188 \\
\hline DRG14 & 0.000 & 0.018 & & & 0.615 & 0.492 \\
\hline DRG15 & 0.005 & 0.069 & & & $-0.494 * *$ & $-0.428 * *$ \\
\hline DRG18 & 0.004 & 0.060 & & & 0.136 & 0.102 \\
\hline DRG19 & 0.003 & 0.054 & & & $-1.163 * * *$ & $-1.076 * * *$ \\
\hline DRG20 & 0.001 & 0.030 & & & $0.785^{* * *}$ & $0.740 * * *$ \\
\hline DRG21 & 0.001 & 0.037 & & & 0.124 & 0.167 \\
\hline DRG22 & 0.001 & 0.026 & & & -0.050 & 0.0273 \\
\hline DRG23 & 0.001 & 0.025 & & & $0.749 * *$ & $0.683^{* *}$ \\
\hline
\end{tabular}




\begin{tabular}{|c|c|c|}
\hline DRG24 & 0.005 & 0.072 \\
\hline DRG25 & 0.001 & 0.032 \\
\hline DRG26 & 0.004 & 0.062 \\
\hline DRG27 & 0.035 & 0.185 \\
\hline DRG28 & 0.008 & 0.087 \\
\hline DRG29 & 0.002 & 0.049 \\
\hline DRG30 & 0.001 & 0.034 \\
\hline DRG31 & 0.040 & 0.196 \\
\hline DRG33 & 0.001 & 0.038 \\
\hline DRG34 & 0.002 & 0.046 \\
\hline DRG35 & 0.005 & 0.071 \\
\hline DRG36 & 0.002 & 0.041 \\
\hline DRG37 & 0.010 & 0.097 \\
\hline DRG38 & 0.001 & 0.024 \\
\hline DRG39 & 0.001 & 0.033 \\
\hline DRG40 & 0.005 & 0.070 \\
\hline DRG41 & 0.004 & 0.065 \\
\hline DRG43 & 0.007 & 0.084 \\
\hline DRG44 & 0.007 & 0.084 \\
\hline DRG45 & 0.002 & 0.042 \\
\hline DRG46 & 0.001 & 0.023 \\
\hline DRG47 & 0.002 & 0.049 \\
\hline DRG49 & 0.001 & 0.035 \\
\hline DRG50 & 0.014 & 0.119 \\
\hline DRG51 & 0.007 & 0.085 \\
\hline DRG52 & 0.001 & 0.033 \\
\hline DRG53 & 0.028 & 0.166 \\
\hline DRG54 & 0.003 & 0.051 \\
\hline DRG55 & 0.004 & 0.063 \\
\hline DRG56 & 0.003 & 0.056 \\
\hline DRG57 & 0.001 & 0.029 \\
\hline DRG59 & 0.002 & 0.046 \\
\hline DRG60 & 0.009 & 0.095 \\
\hline DRG61 & 0.007 & 0.083 \\
\hline DRG62 & 0.004 & 0.067 \\
\hline DRG63 & 0.006 & 0.075 \\
\hline DRG69 & 0.006 & 0.075 \\
\hline DRG70 & 0.023 & 0.151 \\
\hline DRG71 & 0.005 & 0.072 \\
\hline DRG72 & 0.002 & 0.043 \\
\hline DRG73 & 0.004 & 0.065 \\
\hline DRG74 & 0.001 & 0.039 \\
\hline DRG75 & 0.002 & 0.044 \\
\hline
\end{tabular}

\begin{tabular}{|c|c|}
\hline 0.210 & -0.207 \\
\hline $0.708 * * *$ & $0.519 * *$ \\
\hline $0.363^{* *}$ & $-0.340 *$ \\
\hline $552 * * *$ & $0.496 * * *$ \\
\hline $0.789 * * *$ & $0.603 * * *$ \\
\hline 0.082 & -0.028 \\
\hline-0.226 & -0.419 \\
\hline$-0.281 * * *$ & $-0.248 * * *$ \\
\hline $0.523 * * *$ & $0.497 * * *$ \\
\hline$-0.614^{*}$ & -0.499 \\
\hline$-1.193 * * *$ & $-1.123 * * *$ \\
\hline$-0.662 * *$ & $-0.717^{* *}$ \\
\hline $0.292 * * *$ & $0.290 * * *$ \\
\hline 0.288 & 0.141 \\
\hline-0.422 & -0.511 \\
\hline$-0.837 * * *$ & $-0.772 * * *$ \\
\hline$-0.791 * * *$ & $-0.694 * * *$ \\
\hline$-1.286 * * *$ & $-1.162 * * *$ \\
\hline $0.455^{* * *}$ & $0.306 * * *$ \\
\hline $2.079 * * *$ & $1.660 * * *$ \\
\hline $0.933 * * *$ & $0.554 * *$ \\
\hline$-1.098 * * *$ & $-1.053 * * *$ \\
\hline $1.237^{* * *}$ & $1.074^{* * *}$ \\
\hline $0.669 * * *$ & $0.537^{* * *}$ \\
\hline$-1.924 * * *$ & $-1.827 * * *$ \\
\hline $0.472 * *$ & $0.454^{*}$ \\
\hline $0.168 * * *$ & $0.106 * *$ \\
\hline 0.0902 & 0.0832 \\
\hline $0.580 * * *$ & $0.372 * * *$ \\
\hline$-1.535^{* * *}$ & $-1.538 * * *$ \\
\hline$-1.484 * *$ & $-1.509 * *$ \\
\hline$-0.711 * * *$ & $-0.707 * * *$ \\
\hline$-1.520 * * *$ & $-1.495^{* * *}$ \\
\hline$-0.253^{* *}$ & $-0.236 * *$ \\
\hline$-1.323 * * *$ & $-1.266 * * *$ \\
\hline$-0.243^{*}$ & $-0.335^{* *}$ \\
\hline-0.097 & -0.097 \\
\hline-0.013 & -0.034 \\
\hline$-0.331 * *$ & -0.219 \\
\hline$-1.872 * * *$ & $-1.718^{* *}$ \\
\hline 0.056 & 0.047 \\
\hline-0.304 & -0.206 \\
\hline$-1.496 * * *$ & $-1.390 * * *$ \\
\hline
\end{tabular}




\begin{tabular}{|c|c|c|}
\hline DRG76 & 0.002 & 0.050 \\
\hline DRG77 & 0.004 & 0.061 \\
\hline DRG78 & 0.002 & 0.047 \\
\hline DRG79 & 0.000 & 0.013 \\
\hline DRG81 & 0.001 & 0.036 \\
\hline DRG82 & 0.005 & 0.069 \\
\hline DRG83 & 0.016 & 0.125 \\
\hline DRG84 & 0.004 & 0.061 \\
\hline DRG85 & 0.002 & 0.049 \\
\hline DRG86 & 0.001 & 0.031 \\
\hline DRG87 & 0.006 & 0.077 \\
\hline DRG88 & 0.002 & 0.039 \\
\hline DRG89 & 0.003 & 0.057 \\
\hline DRG90 & 0.004 & 0.067 \\
\hline DRG91 & 0.002 & 0.049 \\
\hline DRG92 & 0.007 & 0.081 \\
\hline DRG94 & 0.053 & 0.225 \\
\hline DRG95 & 0.017 & 0.130 \\
\hline DRG96 & 0.001 & 0.023 \\
\hline DRG97 & 0.000 & 0.020 \\
\hline DRG98 & 0.001 & 0.038 \\
\hline DRG99 & 0.006 & 0.075 \\
\hline DRG100 & 0.002 & 0.039 \\
\hline DRG101 & 0.003 & 0.053 \\
\hline DRG102 & 0.000 & 0.014 \\
\hline DRG103 & 0.002 & 0.041 \\
\hline DRG104 & 0.003 & 0.051 \\
\hline DRG105 & 0.001 & 0.023 \\
\hline DRG107 & 0.001 & 0.031 \\
\hline DRG108 & 0.007 & 0.084 \\
\hline DRG109 & 0.003 & 0.053 \\
\hline DRG110 & 0.001 & 0.024 \\
\hline DRG111 & 0.012 & 0.108 \\
\hline DRG112 & 0.004 & 0.061 \\
\hline DRG113 & 0.003 & 0.051 \\
\hline DRG114 & 0.001 & 0.038 \\
\hline DRG115 & 0.002 & 0.044 \\
\hline DRG116 & 0.005 & 0.073 \\
\hline DRG117 & 0.001 & 0.031 \\
\hline DRG118 & 0.001 & 0.038 \\
\hline DRG125 & 0.000 & 0.016 \\
\hline DRG126 & 0.001 & 0.023 \\
\hline DRG127 & 0.005 & 0.068 \\
\hline
\end{tabular}

\begin{tabular}{|c|c|}
\hline 0.036 & -0.054 \\
\hline $0.751 * * *$ & $0.571^{* * *}$ \\
\hline $0.640 * * *$ & $0.547 * * *$ \\
\hline-0.078 & -0.195 \\
\hline-0.299 & -0.212 \\
\hline 0.036 & 0.153 \\
\hline 0.040 & 0.003 \\
\hline-0.052 & -0.084 \\
\hline$-0.820 * * *$ & $-0.833 * * *$ \\
\hline-0.433 & -0.353 \\
\hline$-1.346 * * *$ & $-1.171 * * *$ \\
\hline $0.531 * *$ & 0.104 \\
\hline $0.327^{* *}$ & 0.105 \\
\hline$-1.078 * * *$ & $-0.991 * * *$ \\
\hline$-0.531^{*}$ & $-0.739 * * *$ \\
\hline$-1.086 * * *$ & $-0.993 * * *$ \\
\hline-0.070 & $0.105^{* *}$ \\
\hline $0.855 * * *$ & $0.937 * * *$ \\
\hline $2.243 * * *$ & $1.913^{* * *}$ \\
\hline 0.168 & 0.050 \\
\hline $1.403 * * *$ & $1.475^{* * *}$ \\
\hline $0.496 * * *$ & $0.655^{* * *}$ \\
\hline$-1.236 * *$ & $-1.137 * *$ \\
\hline 0.285 & $0.352^{*}$ \\
\hline $1.692 * * *$ & $1.821 * * *$ \\
\hline $0.460 * *$ & $0.576 * * *$ \\
\hline $0.795 * * *$ & $0.831 * * *$ \\
\hline $1.602 * * *$ & $1.662 * * *$ \\
\hline 0.297 & 0.241 \\
\hline $0.966 * * *$ & $0.895 * * *$ \\
\hline $0.441 * * *$ & $0.393 * *$ \\
\hline $0.800 * * *$ & $0.815^{* * *}$ \\
\hline-0.054 & 0.012 \\
\hline $0.362 * * *$ & $0.341^{* * *}$ \\
\hline 0.025 & 0.039 \\
\hline $0.539 * * *$ & $0.537 * * *$ \\
\hline $0.955 * * *$ & $1.050 * * *$ \\
\hline $0.515 * * *$ & $0.597 * * *$ \\
\hline $0.981 * * *$ & $0.917 * * *$ \\
\hline $0.737 * * *$ & $0.942 * * *$ \\
\hline 0.712 & 0.715 \\
\hline $1.360 * * *$ & $0.630 * * *$ \\
\hline $1.117 * * *$ & $0.700 * * *$ \\
\hline
\end{tabular}




\begin{tabular}{|c|c|c|}
\hline DRG128 & 0.007 & 0.081 \\
\hline DRG129 & 0.002 & 0.041 \\
\hline DRG130 & 0.002 & 0.049 \\
\hline DRG131 & 0.003 & 0.055 \\
\hline DRG133 & 0.001 & 0.029 \\
\hline DRG134 & 0.001 & 0.030 \\
\hline DRG135 & 0.001 & 0.034 \\
\hline DRG136 & 0.005 & 0.071 \\
\hline DRG137 & 0.001 & 0.030 \\
\hline DRG138 & 0.011 & 0.105 \\
\hline DRG139 & 0.001 & 0.036 \\
\hline DRG140 & 0.001 & 0.038 \\
\hline DRG141 & 0.006 & 0.075 \\
\hline DRG142 & 0.001 & 0.035 \\
\hline DRG143 & 0.001 & 0.038 \\
\hline DRG144 & 0.000 & 0.022 \\
\hline DRG145 & 0.008 & 0.089 \\
\hline DRG146 & 0.001 & 0.032 \\
\hline DRG147 & 0.001 & 0.025 \\
\hline DRG148 & 0.004 & 0.063 \\
\hline DRG149 & 0.001 & 0.036 \\
\hline DRG150 & 0.003 & 0.052 \\
\hline DRG151 & 0.001 & 0.038 \\
\hline DRG152 & 0.001 & 0.037 \\
\hline DRG153 & 0.006 & 0.075 \\
\hline DRG155 & 0.001 & 0.030 \\
\hline DRG157 & 0.005 & 0.073 \\
\hline DRG158 & 0.010 & 0.098 \\
\hline DRG159 & 0.005 & 0.072 \\
\hline DRG160 & 0.002 & 0.041 \\
\hline DRG161 & 0.001 & 0.039 \\
\hline DRG162 & 0.001 & 0.037 \\
\hline DRG168 & 0.004 & 0.063 \\
\hline DRG169 & 0.004 & 0.064 \\
\hline DRG170 & 0.002 & 0.044 \\
\hline DRG171 & 0.002 & 0.045 \\
\hline DRG172 & 0.003 & 0.058 \\
\hline DRG173 & 0.000 & 0.018 \\
\hline DRG174 & 0.001 & 0.037 \\
\hline DRG175 & 0.103 & 0.305 \\
\hline DRG176 & 0.002 & 0.041 \\
\hline DRG177 & 0.000 & 0.007 \\
\hline DRG178 & 0.011 & 0.103 \\
\hline
\end{tabular}

\begin{tabular}{|c|c|}
\hline $0.708 * * *$ & $0.540 * * *$ \\
\hline-0.003 & -0.012 \\
\hline$-0.709 * *$ & $-0.633^{* *}$ \\
\hline-0.212 & -0.249 \\
\hline 0.137 & 0.180 \\
\hline$-0.888 * *$ & $-0.837^{*}$ \\
\hline $1.097 * * *$ & $0.748 * * *$ \\
\hline $0.753 * * *$ & $0.571 * * *$ \\
\hline $0.861 * * *$ & $0.682 * * *$ \\
\hline $0.680 * * *$ & $0.648 * * *$ \\
\hline$-1.109 * *$ & $-1.064 * *$ \\
\hline 0.093 & 0.100 \\
\hline $0.672 * * *$ & $0.399 * * *$ \\
\hline$-0.576^{*}$ & -0.394 \\
\hline $0.921 * * *$ & $0.694^{* * *}$ \\
\hline-0.062 & -0.005 \\
\hline$-1.558 * * *$ & $-1.378 * * *$ \\
\hline $0.982 * * *$ & $0.790 * * *$ \\
\hline-0.543 & -0.443 \\
\hline 0.164 & 0.052 \\
\hline-0.138 & -0.147 \\
\hline$-0.432^{*}$ & $-0.505^{*}$ \\
\hline$-0.794 * *$ & $-0.752 * *$ \\
\hline $0.554 * * *$ & $0.560 * * *$ \\
\hline $0.541 * * *$ & $0.598 * * *$ \\
\hline $0.652 * * *$ & 0.377 \\
\hline $0.772 * * *$ & $0.752 * * *$ \\
\hline $1.025^{* * *}$ & $0.902^{* * *}$ \\
\hline-0.186 & -0.103 \\
\hline$-0.600 *$ & $-0.659 * *$ \\
\hline$-1.118^{* *}$ & $-1.074^{* *}$ \\
\hline 0.388 & 0.322 \\
\hline 0.008 & -0.067 \\
\hline 0.213 & $0.257^{*}$ \\
\hline $1.821^{* * *}$ & $1.955^{* * *}$ \\
\hline $0.510 * * *$ & $0.393 * *$ \\
\hline 0.097 & 0.126 \\
\hline $1.704^{* * *}$ & $1.657^{* * *}$ \\
\hline $2.017 * * *$ & $1.775^{* * *}$ \\
\hline $1.141 * * *$ & $1.024 * * *$ \\
\hline-0.075 & -0.233 \\
\hline 0.575 & 0.694 \\
\hline $0.877 * * *$ & $0.889 * * *$ \\
\hline
\end{tabular}




\begin{tabular}{|c|c|c|c|c|c|}
\hline DRG179 & 0.001 & 0.027 & & $1.204 * * *$ & $1.287 * * *$ \\
\hline DRG180 & 0.001 & 0.036 & & $1.564 * * *$ & $1.678 * * *$ \\
\hline DRG181 & 0.000 & 0.015 & & $1.215^{* *}$ & $1.325^{* * *}$ \\
\hline DRG184 & 0.000 & 0.011 & & $1.777 * * *$ & 0.881 \\
\hline DRG185 & 0.001 & 0.033 & & -0.818 & -0.724 \\
\hline Congestive heart failure & 0.095 & 0.293 & & & $0.355^{* * *}$ \\
\hline Valvular disease & 0.038 & 0.192 & & & $-0.085 * *$ \\
\hline Pulmonary circulation disorders & 0.015 & 0.121 & & & $0.246 * * *$ \\
\hline Peripheral vascular disorders & 0.039 & 0.194 & & & $0.734^{* * *}$ \\
\hline Hypertension combined & 0.222 & 0.415 & & & $-0.197 * * *$ \\
\hline Paralysis & 0.000 & 0.006 & & & 0.416 \\
\hline Other neurological disorders & 0.035 & 0.183 & & & $0.606 * * *$ \\
\hline Chronic pulmonary disease & 0.069 & 0.254 & & & $0.0764 * * *$ \\
\hline Diabetes uncomplicated variation & 0.066 & 0.248 & & & 0.001 \\
\hline Diabetes complicated & 0.082 & 0.274 & & & $0.439 * * *$ \\
\hline Hypothyroidism & 0.009 & 0.094 & & & -0.002 \\
\hline Renal failure & 0.090 & 0.286 & & & $0.297 * * *$ \\
\hline Liver disease & 0.014 & 0.117 & & & $0.398 * * *$ \\
\hline $\begin{array}{l}\text { Peptic ulcer disease excluding } \\
\text { bleeding }\end{array}$ & 0.003 & 0.050 & & & 0.125 \\
\hline AIDS & 0.001 & 0.024 & & & $1.326 * * *$ \\
\hline Lymphoma & 0.008 & 0.087 & & & $0.395^{* * *}$ \\
\hline Metastatic cancer & 0.050 & 0.218 & & & $0.505 * * *$ \\
\hline $\begin{array}{l}\text { Solid tumour without metastasis } \\
\text { variation }\end{array}$ & 0.027 & 0.163 & & & $0.166 * * *$ \\
\hline $\begin{array}{l}\text { Rheumatoid arthritis / collagen } \\
\text { vascular diseases }\end{array}$ & 0.015 & 0.122 & & & $0.425^{* * *}$ \\
\hline Obesity & 0.028 & 0.164 & & & $0.408 * * *$ \\
\hline Weight loss & 0.010 & 0.099 & & & $1.120 * * *$ \\
\hline Blood loss anaemia & 0.009 & 0.093 & & & $0.329 * * *$ \\
\hline Deficiency anaemias & 0.016 & 0.126 & & & $0.313^{* * *}$ \\
\hline Alcohol abuse & 0.022 & 0.148 & & & $0.148 * *$ \\
\hline Drug abuse & 0.012 & 0.110 & & & -0.105 \\
\hline Psychoses & 0.007 & 0.084 & & & $0.281 * * *$ \\
\hline Depression & 0.019 & 0.138 & & & $0.287 * * *$ \\
\hline Constant & & & $-4.614 * * *-4.654 * * *$ & $-4.824 * * *$ & $-5.077 * * *$ \\
\hline Observations & & & $1,002,9811,002,981$ & $1,002,981$ & $1,002,981$ \\
\hline Sensitivity & & & 0.771 & 0.688 & 0.709 \\
\hline Specificity & & & 0.465 & 0.650 & 0.683 \\
\hline C-stat (Area under ROC) & & & 0.656 & 0.732 & 0.761 \\
\hline LR test M1 & & & $190 * * *$ & $6471 * * *$ & $9769 * * *$ \\
\hline LR test M2 & & & & $6281 * * *$ & $9580 * * *$ \\
\hline LR test M3 & & & & & $3299 * * *$ \\
\hline
\end{tabular}


Table 27: PSI6

\begin{tabular}{|c|c|c|c|c|c|c|}
\hline Variables & Mean & $\begin{array}{l}\text { Std. } \\
\text { Dev. }\end{array}$ & M1 & M2 & M3 & M4 \\
\hline PSI6 & 0.000 & 0.019 & & & & \\
\hline sex (male) & 0.486 & 0.500 & 0.0234 & 0.0215 & 0.0417 & 0.0737 \\
\hline agecat1 $(<30)$ & 0.101 & 0.301 & $-0.579 * * *$ & $-0.584 * * *$ & $-0.396 * *$ & $-0.357 * *$ \\
\hline agecat2 (30-39) & 0.100 & 0.301 & $-0.300 *$ & $-0.299 *$ & -0.157 & -0.124 \\
\hline agecat3 (40-49) & 0.128 & 0.334 & -0.0786 & -0.0785 & 0.0214 & 0.0300 \\
\hline agecat5 (60-69) & 0.167 & 0.373 & $0.217^{*}$ & $0.219 *$ & 0.129 & 0.0884 \\
\hline agecat6 (70-79) & 0.194 & 0.395 & $0.239 *$ & $0.251^{* *}$ & 0.118 & 0.0581 \\
\hline agecat7 (80+) & 0.165 & 0.371 & $-0.551 * * *$ & $-0.530 * * *$ & $-0.674 * * *$ & $-0.696 * * *$ \\
\hline agesexcat1 & 0.048 & 0.214 & $0.398^{*}$ & $0.402 *$ & 0.287 & 0.248 \\
\hline agesexcat2 & 0.045 & 0.207 & 0.000649 & 0.00247 & -0.0468 & -0.0669 \\
\hline agesexcat3 & 0.061 & 0.239 & -0.313 & -0.312 & -0.347 & $-0.348^{*}$ \\
\hline agesexcat5 & 0.089 & 0.285 & 0.166 & 0.170 & 0.146 & 0.138 \\
\hline agesexcat6 & 0.102 & 0.302 & $0.289^{*}$ & $0.293^{*}$ & 0.238 & 0.179 \\
\hline agesexcat7 & 0.068 & 0.251 & $0.842 * * *$ & $0.844 * * *$ & $0.742 * * *$ & $0.641 * * *$ \\
\hline Maori & 0.134 & 0.340 & & 0.0766 & 0.00182 & 0.00574 \\
\hline Pacific & 0.048 & 0.213 & & $-0.278 * *$ & $-0.395 * * *$ & $-0.376 * * *$ \\
\hline Asian & 0.033 & 0.180 & & -0.0515 & -0.0307 & 0.0167 \\
\hline Other & 0.120 & 0.326 & & -0.108 & -0.102 & -0.0895 \\
\hline NZDep (lower quintile) & 0.134 & 0.340 & & -0.110 & -0.0972 & -0.0910 \\
\hline NZDep (second quintile) & 0.160 & 0.366 & & -0.0274 & -0.0230 & -0.0181 \\
\hline NZDep (fourth quintile) & 0.246 & 0.430 & & 0.0328 & 0.0228 & 0.0192 \\
\hline NZDep (upper quintile) & 0.259 & 0.438 & & $0.171 * *$ & $0.156 * *$ & $0.160 * *$ \\
\hline DRG1 & 0.002 & 0.050 & & & $1.269 * * *$ & $1.230 * * *$ \\
\hline DRG2 & 0.000 & 0.015 & & & $2.760 * * *$ & $2.771 * * *$ \\
\hline DRG3 & 0.002 & 0.040 & & & 0.655 & 0.681 \\
\hline DRG5 & 0.000 & 0.016 & & & $3.242 * * *$ & $2.998 * * *$ \\
\hline DRG6 & 0.017 & 0.130 & & & $1.694^{* * *}$ & $1.284^{* * *}$ \\
\hline DRG7 & 0.002 & 0.049 & & & $2.881 * * *$ & $2.471 * * *$ \\
\hline DRG8 & 0.001 & 0.026 & & & $2.292 * * *$ & $1.538^{* * *}$ \\
\hline DRG9 & 0.020 & 0.140 & & & $1.885^{* * *}$ & $1.720 * * *$ \\
\hline DRG11 & 0.000 & 0.005 & & & $5.850 * * *$ & $5.592 * * *$ \\
\hline DRG12 & 0.000 & 0.013 & & & $4.102 * * *$ & $3.666^{* * *}$ \\
\hline DRG15 & 0.001 & 0.024 & & & 0.433 & 0.478 \\
\hline DRG16 & 0.007 & 0.080 & & & $1.194^{* * *}$ & $1.042^{* * *}$ \\
\hline DRG17 & 0.010 & 0.101 & & & $-1.468 * *$ & $-1.445^{* *}$ \\
\hline DRG18 & 0.012 & 0.110 & & & 0.132 & -0.155 \\
\hline DRG19 & 0.002 & 0.040 & & & $1.154^{* * *}$ & $1.085^{* * *}$ \\
\hline DRG20 & 0.005 & 0.068 & & & 0.117 & 0.0265 \\
\hline DRG24 & 0.006 & 0.076 & & & $1.473 * * *$ & $1.316^{* * *}$ \\
\hline DRG25 & 0.002 & 0.043 & & & $0.881 * *$ & $0.817^{*}$ \\
\hline
\end{tabular}




\begin{tabular}{|c|c|c|c|c|}
\hline DRG26 & 0.001 & 0.029 & $2.760 * * *$ & $2.568 * * *$ \\
\hline DRG27 & 0.001 & 0.037 & 0.730 & 0.621 \\
\hline DRG28 & 0.003 & 0.058 & $0.906^{* * *}$ & $0.681 * *$ \\
\hline DRG29 & 0.003 & 0.057 & -0.187 & -0.246 \\
\hline DRG30 & 0.004 & 0.064 & -1.547 & -1.542 \\
\hline DRG31 & 0.023 & 0.151 & $-0.588 * *$ & $-0.630 * *$ \\
\hline DRG32 & 0.005 & 0.071 & -0.340 & -0.383 \\
\hline DRG33 & 0.001 & 0.025 & $2.485^{* * *}$ & $2.159 * * *$ \\
\hline DRG34 & 0.002 & 0.045 & 0.689 & 0.338 \\
\hline DRG35 & 0.002 & 0.049 & 0.394 & 0.412 \\
\hline DRG36 & 0.015 & 0.123 & $-1.530 * * *$ & $-1.493 * * *$ \\
\hline DRG37 & 0.005 & 0.068 & 0.461 & 0.391 \\
\hline DRG38 & 0.000 & 0.021 & 0.709 & 0.767 \\
\hline DRG39 & 0.003 & 0.052 & -0.503 & -0.444 \\
\hline DRG40 & 0.005 & 0.069 & -0.0611 & -0.152 \\
\hline DRG41 & 0.001 & 0.027 & $2.961 * * *$ & $2.851^{* * *}$ \\
\hline DRG42 & 0.002 & 0.041 & $0.975^{* *}$ & $0.943^{* *}$ \\
\hline DRG43 & 0.001 & 0.035 & 0.344 & 0.604 \\
\hline DRG44 & 0.003 & 0.052 & 0.620 & 0.497 \\
\hline DRG45 & 0.011 & 0.104 & $-1.313^{* *}$ & $-1.309 * *$ \\
\hline DRG46 & 0.014 & 0.116 & -0.409 & -0.348 \\
\hline DRG48 & 0.002 & 0.042 & -0.00421 & -0.0408 \\
\hline DRG49 & 0.004 & 0.063 & $0.971 * * *$ & $0.796 * * *$ \\
\hline DRG50 & 0.004 & 0.064 & $0.727^{* *}$ & $0.742 * *$ \\
\hline DRG51 & 0.007 & 0.086 & 0.0809 & 0.0180 \\
\hline DRG52 & 0.004 & 0.067 & $2.232 * * *$ & $2.204^{* * *}$ \\
\hline DRG53 & 0.033 & 0.178 & $0.927^{* * *}$ & $0.680 * * *$ \\
\hline DRG54 & 0.000 & 0.012 & $1.965^{*}$ & 0.866 \\
\hline Congestive heart failure & 0.035 & 0.183 & & 0.146 \\
\hline Valvular disease & 0.016 & 0.126 & & $0.289 *$ \\
\hline $\begin{array}{l}\text { Pulmonary circulation } \\
\text { disorders }\end{array}$ & 0.005 & 0.069 & & 0.0343 \\
\hline $\begin{array}{l}\text { Peripheral vascular } \\
\text { disorders }\end{array}$ & 0.018 & 0.132 & & $0.385 * *$ \\
\hline Hypertension combined & 0.133 & 0.340 & & 0.0333 \\
\hline Paralysis & 0.026 & 0.161 & & 0.0814 \\
\hline $\begin{array}{l}\text { Other neurological } \\
\text { disorders }\end{array}$ & 0.021 & 0.144 & & -0.0580 \\
\hline $\begin{array}{l}\text { Chronic pulmonary } \\
\text { disease }\end{array}$ & 0.028 & 0.166 & & $1.399^{* * *}$ \\
\hline $\begin{array}{l}\text { Diabetes uncomplicated } \\
\text { variation }\end{array}$ & 0.045 & 0.208 & & $-0.366 * * *$ \\
\hline Diabetes complicated & 0.050 & 0.219 & & $-0.299 * *$ \\
\hline Hypothyroidism & 0.004 & 0.061 & & -0.607 \\
\hline Renal failure & 0.076 & 0.265 & & $-0.290 * * *$ \\
\hline
\end{tabular}




\begin{tabular}{|c|c|c|c|c|c|c|}
\hline Liver disease & 0.008 & 0.088 & & & & $0.694 * * *$ \\
\hline $\begin{array}{l}\text { Peptic ulcer disease } \\
\text { excluding bleeding }\end{array}$ & 0.001 & 0.035 & & & & 0.367 \\
\hline AIDS & 0.000 & 0.019 & & & & 0.950 \\
\hline Lymphoma & 0.008 & 0.088 & & & & 0.0808 \\
\hline Metastatic cancer & 0.027 & 0.162 & & & & $0.318 * * *$ \\
\hline $\begin{array}{l}\text { Solid tumour without } \\
\text { metastasis variation }\end{array}$ & 0.018 & 0.133 & & & & $0.768 * * *$ \\
\hline $\begin{array}{l}\text { Rheumatoid arthritis / } \\
\text { collagen vascular diseases }\end{array}$ & 0.008 & 0.088 & & & & 0.152 \\
\hline Obesity & 0.018 & 0.131 & & & & $-0.466 * *$ \\
\hline Weight loss & 0.004 & 0.063 & & & & $1.022 * * *$ \\
\hline Blood loss anaemia & 0.004 & 0.067 & & & & -0.0142 \\
\hline Deficiency anaemias & 0.007 & 0.081 & & & & 0.240 \\
\hline Alcohol abuse & 0.015 & 0.121 & & & & -0.202 \\
\hline Drug abuse & 0.006 & 0.078 & & & & $0.581 * *$ \\
\hline Psychoses & 0.003 & 0.056 & & & & 0.102 \\
\hline Depression & 0.009 & 0.094 & & & & 0.222 \\
\hline Constant & & & $-7.975 * * *$ & $-8.004 * * *$ & $-8.293 * * *$ & $-8.358 * * *$ \\
\hline Observations & & & $4,374,030$ & $4,374,030$ & $4,374,030$ & $4,374,030$ \\
\hline Sensitivity & & & 0.576 & 0.611 & 0.493 & 0.560 \\
\hline Specificity & & & 0.572 & 0.533 & 0.827 & 0.830 \\
\hline C-stat (Area under ROC) & & & 0.598 & 0.604 & 0.714 & 0.755 \\
\hline LR test M1 & & & & $22 * * *$ & $1299 * * *$ & $1720 * * *$ \\
\hline LR test M2 & & & & & $1277^{* * *}$ & $1697 * * *$ \\
\hline LR test M3 & & & & & & $420 * * *$ \\
\hline
\end{tabular}

*** $p<0.01, * * p<0.05, * p<0.1$ 
Table 28: PSI7

\begin{tabular}{|c|c|c|c|c|c|c|}
\hline Variables & Mean & $\begin{array}{l}\text { Std. } \\
\text { Dev. }\end{array}$ & M1 & M2 & M3 & M4 \\
\hline PSI7 & 0.005 & 0.070 & & & & \\
\hline sex (male) & 0.468 & 0.499 & $0.319 * * *$ & $0.318^{* * *}$ & $0.206^{* * *}$ & $0.192 * * *$ \\
\hline agecat1 $(<30)$ & 0.092 & 0.290 & $-0.771 * * *$ & $-0.769 * * *$ & $-0.764 * * *$ & $-0.653 * * *$ \\
\hline agecat2 (30-39) & 0.089 & 0.284 & $-0.445 * * *$ & $-0.444 * * *$ & $-0.420 * * *$ & $-0.339 * * *$ \\
\hline agecat3 (40-49) & 0.110 & 0.313 & $-0.227 * * *$ & $-0.226 * * *$ & $-0.195 * * *$ & $-0.158^{* *}$ \\
\hline agecat5 (60-69) & 0.151 & 0.358 & -0.0363 & -0.0373 & -0.0196 & -0.0616 \\
\hline agecat6 (70-79) & 0.209 & 0.407 & $-0.0994^{*}$ & $-0.102 *$ & -0.0736 & $-0.147 * * *$ \\
\hline agecat7 $(80+)$ & 0.227 & 0.419 & $-0.339 * * *$ & $-0.343 * * *$ & $-0.307 * * *$ & $-0.387 * * *$ \\
\hline agesexcat1 & 0.047 & 0.212 & $-0.213^{*}$ & $-0.213^{*}$ & $-0.185^{*}$ & -0.169 \\
\hline agesexcat2 & 0.041 & 0.198 & -0.159 & -0.159 & -0.159 & -0.145 \\
\hline agesexcat3 & 0.053 & 0.223 & -0.0267 & -0.0279 & -0.0515 & -0.0386 \\
\hline agesexcat5 & 0.080 & 0.271 & 0.00851 & 0.00805 & 0.0140 & 0.0231 \\
\hline agesexcat6 & 0.102 & 0.302 & -0.0921 & -0.0925 & -0.0545 & -0.0370 \\
\hline agesexcat7 & 0.082 & 0.274 & -0.0158 & -0.0153 & 0.0452 & 0.0731 \\
\hline Maori & 0.119 & 0.324 & & 0.0305 & 0.0329 & -0.0309 \\
\hline Pacific & 0.047 & 0.211 & & 0.00160 & -0.00706 & -0.0783 \\
\hline Asian & 0.028 & 0.164 & & -0.0992 & -0.115 & $-0.134 *$ \\
\hline Other & 0.121 & 0.327 & & $0.0934 * * *$ & $0.0851 * * *$ & $0.0902 * * *$ \\
\hline NZDep (lower quintile) & 0.133 & 0.339 & & 0.00214 & -0.00418 & 0.00500 \\
\hline NZDep (second quintile) & 0.161 & 0.368 & & -0.0480 & -0.0512 & -0.0441 \\
\hline NZDep (fourth quintile) & 0.248 & 0.432 & & -0.0173 & -0.0115 & -0.0164 \\
\hline NZDep (upper quintile) & 0.254 & 0.435 & & -0.0387 & -0.0320 & -0.0417 \\
\hline DRG1 & 0.004 & 0.066 & & & $1.120 * * *$ & $1.148 * * *$ \\
\hline DRG2 & 0.001 & 0.024 & & & -0.485 & -0.418 \\
\hline DRG3 & 0.002 & 0.040 & & & -0.364 & -0.450 \\
\hline DRG4 & 0.002 & 0.042 & & & $-2.525^{* *}$ & $-2.453 * *$ \\
\hline DRG8 & 0.002 & 0.050 & & & 0.0866 & 0.0900 \\
\hline DRG12 & 0.004 & 0.062 & & & $-0.443 * *$ & $-0.433 * *$ \\
\hline DRG13 & 0.001 & 0.035 & & & $0.624 * * *$ & $0.652 * * *$ \\
\hline DRG14 & 0.000 & 0.022 & & & $0.620^{*}$ & $0.603^{*}$ \\
\hline DRG15 & 0.003 & 0.054 & & & -0.0412 & -0.0296 \\
\hline DRG16 & 0.032 & 0.177 & & & $-0.164 * *$ & $-0.157 * *$ \\
\hline DRG17 & 0.001 & 0.031 & & & $-1.116^{*}$ & $-1.372 * *$ \\
\hline DRG18 & 0.038 & 0.192 & & & $-1.085^{* * *}$ & $-1.074 * * *$ \\
\hline DRG20 & 0.010 & 0.099 & & & $-0.840 * * *$ & $-0.794 * * *$ \\
\hline DRG21 & 0.000 & 0.020 & & & 0.0282 & -0.134 \\
\hline DRG22 & 0.001 & 0.023 & & & $1.465^{* * *}$ & $1.027^{* * *}$ \\
\hline DRG23 & 0.003 & 0.051 & & & $0.388^{* *}$ & 0.139 \\
\hline DRG25 & 0.002 & 0.047 & & & $1.411^{* * *}$ & $1.246^{* * *}$ \\
\hline DRG26 & 0.000 & 0.019 & & & 0.630 & 0.392 \\
\hline
\end{tabular}




\begin{tabular}{|c|c|c|}
\hline DRG27 & 0.004 & 0.060 \\
\hline DRG28 & 0.004 & 0.067 \\
\hline DRG29 & 0.001 & 0.028 \\
\hline DRG30 & 0.003 & 0.050 \\
\hline DRG32 & 0.001 & 0.023 \\
\hline DRG33 & 0.010 & 0.102 \\
\hline DRG34 & 0.007 & 0.085 \\
\hline DRG35 & 0.001 & 0.025 \\
\hline DRG36 & 0.020 & 0.141 \\
\hline DRG37 & 0.004 & 0.063 \\
\hline DRG38 & 0.006 & 0.078 \\
\hline DRG39 & 0.002 & 0.047 \\
\hline DRG40 & 0.010 & 0.100 \\
\hline DRG41 & 0.014 & 0.117 \\
\hline DRG42 & 0.005 & 0.068 \\
\hline DRG48 & 0.006 & 0.077 \\
\hline DRG49 & 0.004 & 0.059 \\
\hline DRG50 & 0.002 & 0.046 \\
\hline DRG51 & 0.003 & 0.057 \\
\hline DRG52 & 0.003 & 0.052 \\
\hline DRG53 & 0.001 & 0.038 \\
\hline DRG54 & 0.004 & 0.061 \\
\hline DRG55 & 0.000 & 0.011 \\
\hline DRG56 & 0.002 & 0.040 \\
\hline DRG57 & 0.005 & 0.073 \\
\hline DRG58 & 0.024 & 0.152 \\
\hline DRG59 & 0.004 & 0.064 \\
\hline DRG60 & 0.001 & 0.025 \\
\hline DRG61 & 0.008 & 0.090 \\
\hline DRG62 & 0.000 & 0.007 \\
\hline DRG63 & 0.001 & 0.034 \\
\hline DRG64 & 0.005 & 0.069 \\
\hline DRG65 & 0.002 & 0.048 \\
\hline DRG66 & 0.009 & 0.095 \\
\hline DRG68 & 0.036 & 0.187 \\
\hline DRG69 & 0.011 & 0.102 \\
\hline DRG70 & 0.000 & 0.015 \\
\hline DRG71 & 0.001 & 0.028 \\
\hline DRG72 & 0.004 & 0.067 \\
\hline DRG73 & 0.002 & 0.048 \\
\hline DRG74 & 0.001 & 0.026 \\
\hline DRG75 & 0.004 & 0.059 \\
\hline DRG76 & 0.012 & 0.110 \\
\hline
\end{tabular}

$\begin{array}{ll}0.584 * * * & 0.448 * * * \\ 0.874 * * * & 0.725 * * * \\ 1.039 * * * & 0.783^{* * *} \\ 1.311 * * * & 1.269 * * * \\ 0.832 * * * & 0.754 * * \\ 0.987 * * * & 0.811 * * * \\ 0.518^{* * *} & 0.407 * * * \\ 1.553^{* * *} & 1.306 * * * \\ 0.433^{* * *} & 0.304^{* * *} \\ 0.221 & 0.0868 \\ 0.00117 & -0.0886 \\ 1.371 * * * & 1.295 * * * \\ -0.254 * * & -0.246 * * \\ -0.551 * * * & -0.555 * * \\ 0.865 * * * & 0.765 * * * \\ 1.279 * * * & 1.308 * * * \\ 0.799 * * * & 0.862 * * * \\ 0.906 * * * & 0.899 * * * \\ -0.475 * * & -0.400 * \\ -0.958 * * * & -0.875 * * \\ 0.0751 & 0.0990 \\ -0.134 & -0.191 \\ -0.0237 & -0.0291 \\ 0.382 & 0.419 * \\ -0.0433 & 0.0603 \\ -0.284 * * & -0.225 * * * \\ 0.181 & 0.229 \\ 1.218 * * * & 1.200 * * * \\ -0.627 * * * & -0.551 * * * \\ 2.232 * * * & 2.114 * * * \\ 0.543 * * & 0.0595 \\ 0.319 * * & 0.342 * * * \\ 0.0877 & -0.0533 \\ -0.975 * * * & -0.907 * * * \\ -1.489 * * * & -1.398 * * * \\ -0.839 * * * & -0.796 * * * \\ -0.0298 & -0.184 \\ 0.564 * & 0.602 * * \\ 0.0523 & 0.152 \\ -0.498 * & -0.412 \\ 1.063 * * * & 1.093 * * * \\ -0.263 & -0.236 \\ -0.323 * * * & -0.249 * * \\ & \end{array}$




\begin{tabular}{|c|c|c|c|c|}
\hline DRG78 & 0.001 & 0.037 & $-1.387^{* *}$ & $-1.265^{* *}$ \\
\hline DRG80 & 0.002 & 0.041 & 0.335 & 0.343 \\
\hline DRG81 & 0.002 & 0.041 & 0.186 & 0.268 \\
\hline DRG82 & 0.002 & 0.041 & 0.213 & 0.221 \\
\hline DRG83 & 0.029 & 0.168 & $0.353 * * *$ & $0.395 * * *$ \\
\hline DRG84 & 0.000 & 0.017 & 0.461 & 0.326 \\
\hline DRG85 & 0.003 & 0.058 & 0.112 & -0.0522 \\
\hline DRG86 & 0.003 & 0.057 & 0.258 & 0.174 \\
\hline DRG87 & 0.005 & 0.070 & 0.152 & 0.103 \\
\hline DRG88 & 0.002 & 0.040 & -0.114 & -0.119 \\
\hline DRG89 & 0.002 & 0.049 & $-0.621 * *$ & $-0.551^{*}$ \\
\hline DRG90 & 0.000 & 0.017 & 0.528 & 0.416 \\
\hline DRG91 & 0.002 & 0.041 & $0.403^{*}$ & 0.304 \\
\hline DRG92 & 0.015 & 0.122 & $-0.798 * * *$ & $-0.748 * * *$ \\
\hline DRG93 & 0.004 & 0.060 & $-0.590 * *$ & $-0.633 * * *$ \\
\hline DRG94 & 0.017 & 0.130 & $-1.080 * * *$ & $-1.010 * * *$ \\
\hline DRG98 & 0.005 & 0.069 & $0.274^{* *}$ & $0.254^{*}$ \\
\hline DRG99 & 0.002 & 0.039 & -0.0566 & -0.110 \\
\hline DRG100 & 0.003 & 0.057 & $1.080 * * *$ & $1.105^{* * *}$ \\
\hline DRG101 & 0.005 & 0.068 & $0.877^{* * *}$ & $0.819 * * *$ \\
\hline DRG102 & 0.007 & 0.082 & 0.0339 & 0.118 \\
\hline DRG103 & 0.002 & 0.039 & 0.160 & 0.186 \\
\hline DRG104 & 0.001 & 0.032 & $0.958 * * *$ & $0.983 * * *$ \\
\hline DRG106 & 0.004 & 0.062 & -0.350 & $-0.491 * *$ \\
\hline DRG108 & 0.006 & 0.075 & -0.222 & -0.157 \\
\hline DRG109 & 0.005 & 0.070 & 0.173 & 0.0560 \\
\hline DRG110 & 0.004 & 0.066 & -0.245 & -0.177 \\
\hline DRG111 & 0.000 & 0.005 & $2.450 * * *$ & $2.407 * * *$ \\
\hline DRG112 & 0.001 & 0.029 & $0.561 *$ & 0.468 \\
\hline DRG113 & 0.066 & 0.249 & $-0.364 * * *$ & $-0.453 * * *$ \\
\hline DRG115 & 0.000 & 0.021 & 0.466 & 0.477 \\
\hline DRG116 & 0.001 & 0.029 & -0.712 & -0.691 \\
\hline DRG117 & 0.000 & 0.013 & -0.238 & -0.195 \\
\hline Congestive heart failure & 0.059 & 0.236 & & $0.344^{* * *}$ \\
\hline Valvular disease & 0.027 & 0.163 & & $0.345^{* * *}$ \\
\hline $\begin{array}{l}\text { Pulmonary circulation } \\
\text { disorders }\end{array}$ & 0.009 & 0.093 & & $0.543 * * *$ \\
\hline $\begin{array}{l}\text { Peripheral vascular } \\
\text { disorders }\end{array}$ & 0.024 & 0.153 & & $0.354 * * *$ \\
\hline Hypertension combined & 0.189 & 0.392 & & $0.329 * * *$ \\
\hline Paralysis & 0.050 & 0.217 & & -0.0240 \\
\hline $\begin{array}{l}\text { Other neurological } \\
\text { disorders }\end{array}$ & 0.039 & 0.193 & & $0.211 * * *$ \\
\hline Chronic pulmonary & 0.049 & 0.216 & & 0.0419 \\
\hline
\end{tabular}




\begin{tabular}{|c|c|c|c|c|c|c|}
\hline $\begin{array}{l}\text { Diabetes uncomplicated } \\
\text { variation }\end{array}$ & 0.066 & 0.248 & & & & $-0.110 * *$ \\
\hline Diabetes complicated & 0.046 & 0.209 & & & & 0.0320 \\
\hline Hypothyroidism & 0.006 & 0.078 & & & & -0.110 \\
\hline Renal failure & 0.002 & 0.048 & & & & $0.362 * *$ \\
\hline Liver disease & 0.009 & 0.095 & & & & $0.466 * * *$ \\
\hline $\begin{array}{l}\text { Peptic ulcer disease } \\
\text { excluding bleeding }\end{array}$ & 0.002 & 0.041 & & & & $0.788^{* * *}$ \\
\hline $\begin{array}{l}\text { Rheumatoid arthritis / } \\
\text { collagen vascular } \\
\text { diseases }\end{array}$ & 0.000 & 0.001 & & & & 0.0164 \\
\hline Obesity & 0.011 & 0.105 & & & & $0.284 * * *$ \\
\hline Weight loss & 0.023 & 0.151 & & & & $0.455^{* * *}$ \\
\hline Blood loss anaemia & 0.006 & 0.076 & & & & $0.588 * * *$ \\
\hline Deficiency anaemias & 0.007 & 0.084 & & & & $0.323 * * *$ \\
\hline Alcohol abuse & 0.011 & 0.103 & & & & $0.174 * *$ \\
\hline Drug abuse & 0.021 & 0.145 & & & & 0.104 \\
\hline Psychoses & 0.011 & 0.102 & & & & -0.126 \\
\hline Depression & 0.005 & 0.074 & & & & $0.298 * * *$ \\
\hline Constant & 0.015 & 0.121 & $-5.227^{* * *}$ & $-5.217^{* * *}$ & $-5.195 * * *$ & $-5.330 * * *$ \\
\hline Observations & & & $1,772,919$ & $1,772,919$ & $1,772,919$ & $1,772,919$ \\
\hline Sensitivity & & & 0.619 & 0.632 & 0.657 & 0.598 \\
\hline Specificity & & & 0.491 & 0.480 & 0.563 & 0.655 \\
\hline C-stat (Area under ROC) & & & 0.577 & 0.578 & 0.660 & 0.677 \\
\hline LR test M1 & & & & $15^{* * *}$ & $2438 * * *$ & $3094 * * *$ \\
\hline LR test $M 2$ & & & & & $2424^{* * *}$ & $3079 * * *$ \\
\hline LR test M3 & & & & & & $655^{* * *}$ \\
\hline
\end{tabular}

*** $p<0.01,{ }^{* *} p<0.05,{ }^{*} p<0.1$ 
Table 29: PSI8

\begin{tabular}{|c|c|c|c|c|c|c|}
\hline Variables & Mean & $\begin{array}{l}\text { Std. } \\
\text { Dev. }\end{array}$ & M1 & M2 & M3 & M4 \\
\hline PSI8 & 0.000 & 0.021 & & & & \\
\hline sex (male) & 0.459 & 0.498 & 0.385 & 0.387 & 0.329 & 0.187 \\
\hline agecat1 $(<30)$ & 0.111 & 0.314 & 0.202 & 0.210 & 0.255 & 0.376 \\
\hline agecat3 (40-49) & 0.151 & 0.358 & -0.852 & -0.858 & -0.863 & -0.820 \\
\hline agecat5 (60-69) & 0.164 & 0.370 & $1.783 * * *$ & $1.795 * * *$ & $1.766 * * *$ & $1.620 * * *$ \\
\hline agecat6 (70-79) & 0.185 & 0.388 & $3.359 * * *$ & $3.381 * * *$ & $3.354 * * *$ & $3.105^{* * *}$ \\
\hline agecat7 (80+) & 0.117 & 0.322 & $4.961 * * *$ & $4.991 * * *$ & $4.897 * * *$ & $4.645^{* * *}$ \\
\hline agesexcat1 & 0.044 & 0.206 & -0.677 & -0.686 & -0.686 & -0.623 \\
\hline agesexcat3 & 0.056 & 0.230 & 1.936 & 1.940 & 1.965 & 1.923 \\
\hline agesexcat5 & 0.089 & 0.285 & -0.757 & -0.761 & -0.736 & -0.669 \\
\hline agesexcat6 & 0.103 & 0.304 & -0.586 & -0.593 & -0.583 & -0.458 \\
\hline agesexcat7 & 0.056 & 0.230 & -1.043 & -1.050 & -1.016 & -0.903 \\
\hline Maori & 0.114 & 0.318 & & 0.110 & 0.0449 & -0.213 \\
\hline Pacific & 0.047 & 0.213 & & 0.00519 & 0.0260 & -0.287 \\
\hline Asian & 0.037 & 0.190 & & 0.421 & 0.342 & 0.125 \\
\hline Other & 0.122 & 0.327 & & 0.102 & 0.0623 & 0.0954 \\
\hline NZDep (lower quintile) & 0.132 & 0.339 & & 0.103 & 0.0190 & -0.0525 \\
\hline NZDep (second quintile) & 0.163 & 0.370 & & 0.152 & 0.129 & 0.120 \\
\hline NZDep (fourth quintile) & 0.250 & 0.433 & & -0.181 & -0.155 & -0.165 \\
\hline NZDep (upper quintile) & 0.247 & 0.431 & & -0.103 & -0.118 & -0.117 \\
\hline DRG1 & 0.002 & 0.042 & & & 0.988 & 0.578 \\
\hline DRG2 & 0.004 & 0.065 & & & -0.0607 & -0.749 \\
\hline DRG6 & 0.019 & 0.137 & & & 0.0287 & -0.349 \\
\hline DRG7 & 0.004 & 0.061 & & & 0.107 & -0.267 \\
\hline DRG8 & 0.007 & 0.085 & & & $2.005^{* * *}$ & $1.590 * * *$ \\
\hline DRG9 & 0.007 & 0.080 & & & $3.919 * * *$ & $3.578 * * *$ \\
\hline Congestive heart failure & 0.015 & 0.122 & & & & $0.925^{* * *}$ \\
\hline Valvular disease & 0.011 & 0.103 & & & & $0.533^{* *}$ \\
\hline $\begin{array}{l}\text { Pulmonary circulation } \\
\text { disorders }\end{array}$ & 0.003 & 0.051 & & & & 0.572 \\
\hline $\begin{array}{l}\text { Peripheral vascular } \\
\text { disorders }\end{array}$ & 0.021 & 0.142 & & & & -0.0218 \\
\hline Hypertension combined & 0.109 & 0.311 & & & & $0.298 * *$ \\
\hline Paralysis & 0.008 & 0.087 & & & & -0.158 \\
\hline $\begin{array}{l}\text { Other neurological } \\
\text { disorders }\end{array}$ & 0.005 & 0.071 & & & & $1.874^{* * *}$ \\
\hline Chronic pulmonary disease & 0.014 & 0.118 & & & & $0.714^{* * *}$ \\
\hline $\begin{array}{l}\text { Diabetes uncomplicated } \\
\text { variation }\end{array}$ & 0.035 & 0.184 & & & & $0.452 * *$ \\
\hline Diabetes complicated & 0.040 & 0.196 & & & & 0.224 \\
\hline Hypothyroidism & 0.002 & 0.039 & & & & 0.0752 \\
\hline Renal failure & 0.031 & 0.173 & & & & $0.807 * * *$ \\
\hline
\end{tabular}




\begin{tabular}{|c|c|c|c|c|c|c|}
\hline Liver disease & 0.004 & 0.066 & & & & $1.296 * * *$ \\
\hline $\begin{array}{l}\text { Peptic ulcer disease } \\
\text { excluding bleeding }\end{array}$ & 0.001 & 0.023 & & & & $1.040^{*}$ \\
\hline $\begin{array}{l}\text { Solid tumour without } \\
\text { metastasis variation }\end{array}$ & 0.000 & 0.015 & & & & -0.112 \\
\hline $\begin{array}{l}\text { Rheumatoid arthritis / } \\
\text { collagen vascular diseases }\end{array}$ & 0.011 & 0.102 & & & & 0.181 \\
\hline Obesity & 0.003 & 0.056 & & & & -0.584 \\
\hline Weight loss & 0.016 & 0.124 & & & & $1.515^{* * *}$ \\
\hline Blood loss anaemia & 0.002 & 0.039 & & & & $1.155^{* * *}$ \\
\hline Deficiency anaemias & 0.004 & 0.061 & & & & $0.875^{* * *}$ \\
\hline Alcohol abuse & 0.003 & 0.055 & & & & $1.186^{* * *}$ \\
\hline Drug abuse & 0.003 & 0.054 & & & & 1.081 \\
\hline Psychoses & 0.002 & 0.046 & & & & $1.379 * *$ \\
\hline Depression & 0.001 & 0.032 & & & & $0.819 * *$ \\
\hline Constant & 0.002 & 0.043 & $-10.64 * * *$ & $-10.67 * * *$ & $-10.92 * * *$ & $-10.97 * * *$ \\
\hline Observations & & & $1,032,261$ & $1,032,261$ & $1,032,261$ & $1,032,261$ \\
\hline Sensitivity & & & 0.920 & 0.920 & 0.829 & 0.873 \\
\hline Specificity & & & 0.698 & 0.698 & 0.805 & 0.839 \\
\hline C-stat (Area under ROC) & & & 0.876 & 0.878 & 0.905 & 0.933 \\
\hline LR test M1 & & & & 10.000 & $743 * * *$ & $1067^{* * *}$ \\
\hline LR test $\mathrm{M} 2$ & & & & & $734^{* * *}$ & $1057^{* * *}$ \\
\hline LR test M3 & & & & & & $323 * * *$ \\
\hline
\end{tabular}

*** $p<0.01,{ }^{* *} p<0.05, * p<0.1$ 
Table 30: PSI9

\begin{tabular}{|c|c|c|c|c|c|c|}
\hline Variables & Mean & $\begin{array}{l}\text { Std. } \\
\text { Dev. }\end{array}$ & M1 & M2 & M3 & M4 \\
\hline PSI9 & 0.019 & 0.136 & & & & \\
\hline sex (male) & 0.482 & 0.500 & $0.220 * * *$ & $0.228 * * *$ & $0.180 * * *$ & $0.154 * * *$ \\
\hline agecat1 $(<30)$ & 0.123 & 0.328 & $-0.711 * * *$ & $-0.741 * * *$ & $-0.526 * * *$ & $-0.419 * * *$ \\
\hline agecat2 (30-39) & 0.122 & 0.327 & $-0.368 * * *$ & $-0.398 * * *$ & $-0.272 * * *$ & $-0.188 * * *$ \\
\hline agecat3 (40-49) & 0.146 & 0.353 & $-0.0981 * * *$ & $-0.116 * * *$ & $-0.0995 * * *$ & $-0.0599 *$ \\
\hline agecat5 (60-69) & 0.163 & 0.370 & $0.228 * * *$ & $0.246 * * *$ & $0.202 * * *$ & $0.154^{* * *}$ \\
\hline agecat6 (70-79) & 0.180 & 0.384 & $0.360 * * *$ & $0.407^{* * *}$ & $0.367 * * *$ & $0.274^{* * *}$ \\
\hline agecat7 (80+) & 0.119 & 0.324 & $0.313^{* * *}$ & $0.379 * * *$ & $0.426 * * *$ & $0.298 * * *$ \\
\hline agesexcat1 & 0.065 & 0.246 & $0.136 * *$ & $0.116^{*}$ & $0.157^{* *}$ & $0.182 * * *$ \\
\hline agesexcat2 & 0.051 & 0.220 & $-0.169 * * *$ & $-0.174 * * *$ & -0.0621 & -0.0417 \\
\hline agesexcat3 & 0.062 & 0.242 & $-0.179 * * *$ & $-0.180 * * *$ & -0.0492 & -0.0288 \\
\hline agesexcat5 & 0.086 & 0.281 & 0.0389 & 0.0378 & -0.0392 & -0.0300 \\
\hline agesexcat6 & 0.096 & 0.294 & 0.0559 & 0.0521 & -0.000156 & 0.0103 \\
\hline agesexcat7 & 0.051 & 0.220 & 0.0591 & 0.0519 & 0.0469 & 0.0642 \\
\hline Maori & 0.114 & 0.318 & & $0.319 * * *$ & $0.268^{* * *}$ & $0.183^{* * *}$ \\
\hline Pacific & 0.046 & 0.210 & & $0.419 * * *$ & $0.377^{* * *}$ & $0.284 * * *$ \\
\hline Asian & 0.034 & 0.181 & & $0.185^{* * *}$ & $0.172 * * *$ & $0.151^{* * *}$ \\
\hline Other & 0.123 & 0.328 & & -0.0267 & $-0.0399 * *$ & -0.0290 \\
\hline NZDep (lower quintile) & 0.137 & 0.343 & & 0.0263 & 0.0141 & 0.0230 \\
\hline $\begin{array}{l}\text { NZDep (second } \\
\text { quintile) }\end{array}$ & 0.165 & 0.371 & & 0.0287 & 0.0178 & 0.0259 \\
\hline NZDep (fourth quintile) & 0.248 & 0.432 & & -0.0278 & -0.0183 & -0.0202 \\
\hline NZDep (upper quintile) & 0.244 & 0.429 & & $-0.0550 * * *$ & $-0.0318^{*}$ & $-0.0403^{* *}$ \\
\hline DRG1 & 0.008 & 0.088 & & & $1.378 * * *$ & $1.193 * * *$ \\
\hline DRG2 & 0.002 & 0.049 & & & $1.498 * * *$ & $1.174 * * *$ \\
\hline DRG3 & 0.005 & 0.070 & & & $1.049 * * *$ & $1.084 * * *$ \\
\hline DRG4 & 0.000 & 0.022 & & & $1.822^{* * *}$ & $1.804^{* * *}$ \\
\hline DRG5 & 0.002 & 0.040 & & & $0.918 * * *$ & $1.012^{* * *}$ \\
\hline DRG7 & 0.004 & 0.067 & & & $0.711^{* * *}$ & $0.608 * * *$ \\
\hline DRG8 & 0.002 & 0.043 & & & -0.0927 & -0.190 \\
\hline DRG9 & 0.001 & 0.028 & & & $2.747^{* * *}$ & $1.805^{* * *}$ \\
\hline DRG10 & 0.004 & 0.061 & & & $2.411^{* * *}$ & $1.796 * * *$ \\
\hline DRG11 & 0.003 & 0.056 & & & $2.244^{* * *}$ & $1.823^{* * *}$ \\
\hline DRG12 & 0.001 & 0.022 & & & $2.452 * * *$ & $1.880 * * *$ \\
\hline DRG13 & 0.005 & 0.072 & & & $1.897 * * *$ & $1.527^{* * *}$ \\
\hline DRG14 & 0.007 & 0.081 & & & $2.110 * * *$ & $1.707^{* * *}$ \\
\hline DRG15 & 0.001 & 0.037 & & & $1.073 * * *$ & $0.412 * * *$ \\
\hline DRG17 & 0.002 & 0.042 & & & $0.917^{* * *}$ & $0.485^{* * *}$ \\
\hline DRG22 & 0.004 & 0.065 & & & $1.313^{* * *}$ & $1.232 * * *$ \\
\hline DRG23 & 0.019 & 0.136 & & & $1.104^{* * *}$ & $1.011^{* * *}$ \\
\hline DRG24 & 0.006 & 0.076 & & & $0.616 * * *$ & $0.577^{* * *}$ \\
\hline
\end{tabular}




\begin{tabular}{|c|c|c|c|c|}
\hline DRG25 & 0.004 & 0.067 & $0.810 * * *$ & $0.642 * * *$ \\
\hline DRG26 & 0.015 & 0.122 & $-0.507 * * *$ & $-0.445 * * *$ \\
\hline DRG27 & 0.010 & 0.098 & -0.0219 & 0.0212 \\
\hline DRG28 & 0.014 & 0.117 & $-0.409 * * *$ & $-0.291 * * *$ \\
\hline DRG29 & 0.004 & 0.060 & -0.0980 & -0.0724 \\
\hline DRG30 & 0.015 & 0.123 & $-0.792 * * *$ & $-0.748 * * *$ \\
\hline DRG31 & 0.004 & 0.065 & $0.474 * * *$ & $0.384 * * *$ \\
\hline DRG32 & 0.002 & 0.045 & $1.819 * * *$ & $1.611^{* * *}$ \\
\hline DRG33 & 0.021 & 0.144 & 0.0794 & $0.122 * *$ \\
\hline DRG35 & 0.048 & 0.213 & $0.541 * * *$ & $0.526^{* * *}$ \\
\hline DRG36 & 0.015 & 0.121 & $0.450 * * *$ & $0.317^{* * *}$ \\
\hline DRG37 & 0.001 & 0.036 & $1.638^{* * *}$ & $1.568 * * *$ \\
\hline DRG38 & 0.006 & 0.078 & -0.0637 & -0.0523 \\
\hline DRG39 & 0.012 & 0.107 & $0.189 * * *$ & $0.236 * * *$ \\
\hline DRG40 & 0.002 & 0.042 & $-1.089 * * *$ & $-1.000 * * *$ \\
\hline DRG41 & 0.003 & 0.055 & $-2.359 * * *$ & $-2.286 * * *$ \\
\hline DRG43 & 0.010 & 0.098 & $1.331^{* * *}$ & $1.401 * * *$ \\
\hline DRG44 & 0.013 & 0.115 & $0.399 * * *$ & $0.496 * * *$ \\
\hline DRG46 & 0.003 & 0.053 & $0.745^{* * *}$ & $0.599 * * *$ \\
\hline DRG47 & 0.020 & 0.141 & -0.000284 & $0.135 * * *$ \\
\hline DRG48 & 0.009 & 0.094 & $-0.722 * * *$ & $-0.557 * * *$ \\
\hline DRG49 & 0.053 & 0.225 & $-1.500 * * *$ & $-1.337 * * *$ \\
\hline DRG50 & 0.000 & 0.020 & $0.941 * * *$ & $0.706 * * *$ \\
\hline DRG51 & 0.004 & 0.063 & $0.855^{* * *}$ & $0.908 * * *$ \\
\hline DRG52 & 0.001 & 0.025 & $2.174 * * *$ & $1.735^{* * *}$ \\
\hline DRG53 & 0.002 & 0.048 & $1.877 * * *$ & $1.796 * * *$ \\
\hline DRG54 & 0.005 & 0.072 & $0.864^{* * *}$ & $0.790 * * *$ \\
\hline DRG55 & 0.003 & 0.056 & $2.345^{* * *}$ & $2.369 * * *$ \\
\hline DRG56 & 0.004 & 0.061 & $0.652 * * *$ & 0.126 \\
\hline DRG57 & 0.002 & 0.048 & $1.672 * * *$ & $1.794^{* * *}$ \\
\hline DRG58 & 0.009 & 0.094 & $0.930 * * *$ & $1.035^{* * *}$ \\
\hline DRG59 & 0.002 & 0.045 & $1.104^{* * *}$ & $1.061^{* * *}$ \\
\hline DRG60 & 0.007 & 0.085 & $0.146^{*}$ & $0.244^{* * *}$ \\
\hline DRG61 & 0.035 & 0.185 & $0.783 * * *$ & $0.813^{* * *}$ \\
\hline DRG62 & 0.020 & 0.140 & $-0.129 *$ & -0.0668 \\
\hline DRG63 & 0.000 & 0.019 & $1.588 * * *$ & $1.489 * * *$ \\
\hline DRG64 & 0.002 & 0.048 & $1.305^{* * *}$ & $1.213^{* * *}$ \\
\hline DRG65 & 0.006 & 0.076 & $1.732 * * *$ & $1.614^{* * *}$ \\
\hline DRG67 & 0.011 & 0.106 & $1.105^{* * *}$ & $1.097 * * *$ \\
\hline DRG68 & 0.005 & 0.070 & $0.213^{* *}$ & 0.0647 \\
\hline DRG69 & 0.001 & 0.034 & $2.047 * * *$ & $1.969 * * *$ \\
\hline DRG70 & 0.000 & 0.013 & $3.183 * * *$ & $2.116^{* * *}$ \\
\hline $\begin{array}{l}\text { Congestive heart } \\
\text { failure }\end{array}$ & 0.016 & 0.124 & & $0.359 * * *$ \\
\hline
\end{tabular}




\begin{tabular}{|c|c|c|c|c|c|c|}
\hline Valvular disease & 0.010 & 0.099 & & & & $0.791 * * *$ \\
\hline $\begin{array}{l}\text { Pulmonary circulation } \\
\text { disorders }\end{array}$ & 0.003 & 0.054 & & & & $0.795^{* * *}$ \\
\hline $\begin{array}{l}\text { Peripheral vascular } \\
\text { disorders }\end{array}$ & 0.017 & 0.130 & & & & $0.549 * * *$ \\
\hline $\begin{array}{l}\text { Hypertension } \\
\text { combined }\end{array}$ & 0.103 & 0.304 & & & & $0.507 * * *$ \\
\hline Paralysis & 0.009 & 0.096 & & & & $0.541^{* * *}$ \\
\hline $\begin{array}{l}\text { Other neurological } \\
\text { disorders }\end{array}$ & 0.007 & 0.083 & & & & $0.192 * * *$ \\
\hline $\begin{array}{l}\text { Chronic pulmonary } \\
\text { disease }\end{array}$ & 0.015 & 0.121 & & & & $0.171 * * *$ \\
\hline $\begin{array}{l}\text { Diabetes } \\
\text { uncomplicated } \\
\text { variation }\end{array}$ & 0.036 & 0.186 & & & & $-0.119 * * *$ \\
\hline Diabetes complicated & 0.035 & 0.184 & & & & $-0.238 * * *$ \\
\hline Hypothyroidism & 0.002 & 0.043 & & & & $0.292 * * *$ \\
\hline Renal failure & 0.028 & 0.164 & & & & $0.403 * * *$ \\
\hline Liver disease & 0.004 & 0.065 & & & & $0.781 * * *$ \\
\hline $\begin{array}{l}\text { Peptic ulcer disease } \\
\text { excluding bleeding }\end{array}$ & 0.001 & 0.023 & & & & $0.615^{* * *}$ \\
\hline $\begin{array}{l}\text { Solid tumour without } \\
\text { metastasis variation }\end{array}$ & 0.012 & 0.111 & & & & $0.322 * * *$ \\
\hline $\begin{array}{l}\text { Rheumatoid arthritis / } \\
\text { collagen vascular } \\
\text { diseases }\end{array}$ & 0.005 & 0.069 & & & & $0.126^{*}$ \\
\hline Obesity & 0.014 & 0.119 & & & & $0.287 * * *$ \\
\hline Weight loss & 0.002 & 0.039 & & & & $0.745^{* * *}$ \\
\hline Blood loss anaemia & 0.003 & 0.059 & & & & $1.097 * * *$ \\
\hline Deficiency anaemias & 0.003 & 0.057 & & & & $0.223 * * *$ \\
\hline Alcohol abuse & 0.006 & 0.080 & & & & $0.450 * * *$ \\
\hline Drug abuse & 0.003 & 0.054 & & & & $0.302 * * *$ \\
\hline Psychoses & 0.001 & 0.037 & & & & $0.539 * * *$ \\
\hline Depression & 0.003 & 0.051 & & & & $0.527 * * *$ \\
\hline Constant & & & $-4.123 * * *$ & $-4.188 * * *$ & $-4.583 * * *$ & $-4.703 * * *$ \\
\hline Observations & & & $1,418,513$ & $1,418,513$ & $1,418,513$ & $1,418,513$ \\
\hline Sensitivity & & & 0.685 & 0.646 & 0.566 & 0.640 \\
\hline Specificity & & & 0.469 & 0.515 & 0.743 & 0.745 \\
\hline $\begin{array}{l}\text { C-stat (Area under } \\
\text { ROC) }\end{array}$ & & & 0.601 & 0.607 & 0.720 & 0.754 \\
\hline LR test M1 & & & & $391^{* * *}$ & $16619 * * *$ & $20832 * * *$ \\
\hline LR test M2 & & & & & $16228 * * *$ & $20441 * * *$ \\
\hline LR test M3 & & & & & & $4213^{* * *}$ \\
\hline
\end{tabular}

${ }^{* * *} p<0.01,{ }^{* *} p<0.05,{ }^{*} p<0.1$ 
Table 31: PSI10

\begin{tabular}{|c|c|c|c|c|c|c|}
\hline Variables & Mean & $\begin{array}{l}\text { Std. } \\
\text { Dev. }\end{array}$ & M1 & M2 & M3 & M4 \\
\hline PSI10 & 0.002 & 0.042 & & & & \\
\hline sex (male) & 0.542 & 0.498 & 0.425 & 0.433 & 0.347 & 0.170 \\
\hline agecat1 $(<30)$ & 0.111 & 0.314 & -0.840 & $-0.932 *$ & -0.876 & -0.301 \\
\hline agecat2 (30-39) & 0.105 & 0.306 & $-1.787^{* *}$ & $-1.857 * *$ & $-1.801 * *$ & $-1.358^{*}$ \\
\hline agecat3 (40-49) & 0.139 & 0.346 & -0.475 & -0.501 & -0.476 & -0.205 \\
\hline agecat5 (60-69) & 0.191 & 0.393 & 0.183 & 0.231 & 0.210 & -0.0177 \\
\hline agecat6 (70-79) & 0.187 & 0.390 & -0.125 & 0.0193 & -0.0191 & -0.311 \\
\hline agecat7 (80+) & 0.099 & 0.299 & $-1.152^{* *}$ & $-0.917 *$ & -0.884 & $-1.167 * *$ \\
\hline agesexcat1 & 0.071 & 0.256 & -0.777 & -0.829 & -0.686 & -0.441 \\
\hline agesexcat2 & 0.054 & 0.225 & 1.144 & 1.117 & 1.188 & $1.458^{*}$ \\
\hline agesexcat3 & 0.070 & 0.255 & -0.173 & -0.200 & -0.170 & -0.174 \\
\hline agesexcat5 & 0.109 & 0.311 & -0.147 & -0.133 & -0.111 & 0.0486 \\
\hline agesexcat6 & 0.104 & 0.306 & 0.178 & 0.189 & 0.243 & 0.352 \\
\hline agesexcat7 & 0.045 & 0.207 & 0.780 & 0.765 & 0.807 & 0.783 \\
\hline Maori & 0.123 & 0.328 & & $0.798 * * *$ & $0.760 * * *$ & 0.164 \\
\hline Pacific & 0.047 & 0.213 & & $0.989 * * *$ & $0.924 * * *$ & 0.371 \\
\hline Asian & 0.032 & 0.176 & & 0.142 & 0.147 & -0.237 \\
\hline Other & 0.123 & 0.329 & & -0.0295 & -0.0217 & 0.0135 \\
\hline NZDep (lower quintile) & 0.138 & 0.345 & & -0.116 & -0.145 & -0.0631 \\
\hline NZDep (second quintile) & 0.165 & 0.371 & & -0.293 & -0.307 & -0.189 \\
\hline NZDep (fourth quintile) & 0.242 & 0.428 & & 0.0495 & 0.0467 & 0.0375 \\
\hline NZDep (upper quintile) & 0.248 & 0.432 & & 0.0905 & 0.0856 & 0.0102 \\
\hline DRG1 & 0.010 & 0.101 & & & -0.733 & -1.004 \\
\hline DRG2 & 0.002 & 0.047 & & & $2.038 * * *$ & 0.366 \\
\hline DRG3 & 0.010 & 0.100 & & & $1.666^{* * *}$ & 0.431 \\
\hline DRG4 & 0.010 & 0.097 & & & $1.093 * * *$ & 0.511 \\
\hline DRG5 & 0.001 & 0.033 & & & $2.750 * * *$ & $1.940 * * *$ \\
\hline DRG6 & 0.021 & 0.142 & & & 0.294 & -0.234 \\
\hline DRG7 & 0.006 & 0.078 & & & $1.779 * * *$ & $1.290 * * *$ \\
\hline DRG8 & 0.002 & 0.049 & & & 0.641 & -0.278 \\
\hline DRG9 & 0.004 & 0.066 & & & 0.655 & -0.394 \\
\hline DRG11 & 0.017 & 0.129 & & & 0.305 & 0.0113 \\
\hline DRG14 & 0.001 & 0.022 & & & $3.096 * * *$ & $1.653^{* *}$ \\
\hline DRG16 & 0.008 & 0.087 & & & 0.383 & 0.428 \\
\hline DRG17 & 0.012 & 0.107 & & & 0.447 & -0.710 \\
\hline DRG18 & 0.008 & 0.092 & & & $1.607 * * *$ & $1.246 * * *$ \\
\hline DRG19 & 0.008 & 0.090 & & & $1.663 * * *$ & $1.533^{* * *}$ \\
\hline Congestive heart failure & 0.025 & 0.155 & & & & $1.231 * * *$ \\
\hline Valvular disease & 0.019 & 0.136 & & & & $0.769 * * *$ \\
\hline $\begin{array}{l}\text { Pulmonary circulation } \\
\text { disorders }\end{array}$ & 0.005 & 0.073 & & & & $1.359 * * *$ \\
\hline
\end{tabular}




\begin{tabular}{|c|c|c|c|c|c|c|}
\hline $\begin{array}{l}\text { Peripheral vascular } \\
\text { disorders }\end{array}$ & 0.038 & 0.192 & & & & 0.0911 \\
\hline Hypertension combined & 0.156 & 0.363 & & & & $0.786 * * *$ \\
\hline Paralysis & 0.014 & 0.116 & & & & 0.501 \\
\hline $\begin{array}{l}\text { Other neurological } \\
\text { disorders }\end{array}$ & 0.008 & 0.088 & & & & -0.539 \\
\hline $\begin{array}{l}\text { Chronic pulmonary } \\
\text { disease }\end{array}$ & 0.021 & 0.143 & & & & $0.837 * * *$ \\
\hline $\begin{array}{l}\text { Diabetes uncomplicated } \\
\text { variation }\end{array}$ & 0.039 & 0.194 & & & & -0.005 \\
\hline Diabetes complicated & 0.056 & 0.231 & & & & $0.509 * * *$ \\
\hline Hypothyroidism & 0.003 & 0.051 & & & & $1.269 * *$ \\
\hline Renal failure & 0.064 & 0.245 & & & & $1.254^{* * *}$ \\
\hline Liver disease & 0.007 & 0.084 & & & & $2.014 * * *$ \\
\hline $\begin{array}{l}\text { Peptic ulcer disease } \\
\text { excluding bleeding }\end{array}$ & 0.001 & 0.024 & & & & $1.908 * *$ \\
\hline AIDS & 0.000 & 0.022 & & & & $2.082^{*}$ \\
\hline Lymphoma & 0.004 & 0.062 & & & & $1.176^{*}$ \\
\hline Metastatic cancer & 0.043 & 0.204 & & & & 0.0191 \\
\hline $\begin{array}{l}\text { Solid tumour without } \\
\text { metastasis variation }\end{array}$ & 0.024 & 0.154 & & & & 0.642 \\
\hline $\begin{array}{l}\text { Rheumatoid arthritis / } \\
\text { collagen vascular diseases }\end{array}$ & 0.006 & 0.074 & & & & $1.198 * * *$ \\
\hline Obesity & 0.017 & 0.130 & & & & $0.467^{*}$ \\
\hline Weight loss & 0.003 & 0.057 & & & & $1.875^{* * *}$ \\
\hline Blood loss anaemia & 0.003 & 0.053 & & & & -0.468 \\
\hline Deficiency anaemias & 0.004 & 0.065 & & & & 0.294 \\
\hline Alcohol abuse & 0.004 & 0.066 & & & & 0.590 \\
\hline Depression & 0.005 & 0.070 & & & & $1.137^{* *}$ \\
\hline Constant & & & $-6.299 * * *$ & $-6.528 * * *$ & $-6.701 * * *$ & $-7.353 * * *$ \\
\hline Observations & & & 126,531 & 126,531 & 126,531 & 126,531 \\
\hline Sensitivity & & & 0.715 & 0.706 & 0.623 & 0.776 \\
\hline Specificity & & & 0.492 & 0.556 & 0.680 & 0.853 \\
\hline C-stat (Area under ROC) & & & 0.639 & 0.675 & 0.719 & 0.881 \\
\hline LR test M1 & & & & $37 * * *$ & $124^{* * *}$ & $537 * * *$ \\
\hline LR test M2 & & & & & $87^{* * *}$ & $499 * * *$ \\
\hline LR test $M 3$ & & & & & & $413^{* * *}$ \\
\hline
\end{tabular}

*** $p<0.01,{ }^{* *} p<0.05, * p<0.1$ 
Table 32: PSI11

\begin{tabular}{|c|c|c|c|c|c|c|}
\hline Variables & Mean & $\begin{array}{l}\text { Std. } \\
\text { Dev. }\end{array}$ & M1 & M2 & M3 & M4 \\
\hline PSI11 & 0.001 & 0.038 & & & & \\
\hline sex (male) & 0.510 & 0.500 & 0.273 & 0.282 & 0.240 & 0.218 \\
\hline agecat1 $(<30)$ & 0.144 & 0.351 & -0.621 & -0.641 & -0.612 & -0.215 \\
\hline agecat2 (30-39) & 0.131 & 0.337 & -0.860 & -0.895 & -0.867 & -0.551 \\
\hline agecat3 (40-49) & 0.156 & 0.363 & -0.596 & -0.608 & -0.603 & -0.446 \\
\hline agecat5 (60-69) & 0.162 & 0.368 & $0.738^{*}$ & $0.746^{*}$ & $0.741^{*}$ & 0.678 \\
\hline agecat6 (70-79) & 0.154 & 0.361 & -0.161 & -0.142 & -0.136 & -0.758 \\
\hline agecat7 (80+) & 0.095 & 0.293 & 0.148 & 0.187 & 0.207 & -0.289 \\
\hline agesexcat1 & 0.093 & 0.290 & -0.0221 & -0.0133 & 0.0550 & 0.101 \\
\hline agesexcat2 & 0.066 & 0.248 & 0.557 & 0.580 & 0.627 & 0.646 \\
\hline agesexcat3 & 0.073 & 0.260 & 0.330 & 0.331 & 0.369 & 0.433 \\
\hline agesexcat5 & 0.083 & 0.275 & -0.167 & -0.172 & -0.147 & -0.160 \\
\hline agesexcat6 & 0.079 & 0.270 & 0.728 & 0.725 & 0.761 & $1.104^{*}$ \\
\hline agesexcat7 & 0.040 & 0.195 & -0.281 & -0.294 & -0.264 & -0.487 \\
\hline Maori & 0.125 & 0.330 & & 0.278 & 0.282 & -0.236 \\
\hline Pacific & 0.048 & 0.213 & & -0.440 & -0.441 & -0.765 \\
\hline Asian & 0.032 & 0.176 & & $0.735^{*}$ & $0.737^{*}$ & 0.662 \\
\hline Other & 0.119 & 0.324 & & -0.000667 & 0.000936 & 0.0485 \\
\hline NZDep (lower quintile) & 0.139 & 0.346 & & -0.107 & -0.115 & -0.100 \\
\hline NZDep (second quintile) & 0.167 & 0.373 & & 0.267 & 0.262 & 0.329 \\
\hline NZDep (fourth quintile) & 0.241 & 0.428 & & 0.149 & 0.155 & 0.166 \\
\hline NZDep (upper quintile) & 0.243 & 0.429 & & -0.0538 & -0.0327 & -0.127 \\
\hline DRG1 & 0.017 & 0.128 & & & -0.742 & -1.024 \\
\hline DRG2 & 0.001 & 0.037 & & & $1.886^{*}$ & $1.793^{*}$ \\
\hline DRG8 & 0.005 & 0.071 & & & 0.902 & 1.001 \\
\hline DRG9 & 0.023 & 0.150 & & & $0.729 *$ & 0.551 \\
\hline DRG13 & 0.009 & 0.096 & & & -0.102 & 0.126 \\
\hline DRG21 & 0.013 & 0.113 & & & -0.650 & -1.100 \\
\hline DRG29 & 0.014 & 0.119 & & & -0.444 & -0.430 \\
\hline DRG38 & 0.013 & 0.114 & & & -0.599 & -0.299 \\
\hline DRG43 & 0.002 & 0.041 & & & 1.330 & 1.171 \\
\hline DRG45 & 0.004 & 0.064 & & & 0.389 & 0.637 \\
\hline DRG46 & 0.011 & 0.104 & & & -0.358 & -0.00493 \\
\hline DRG47 & 0.005 & 0.070 & & & 0.892 & $1.248^{*}$ \\
\hline DRG55 & 0.003 & 0.054 & & & 1.164 & 1.660 \\
\hline DRG61 & 0.012 & 0.107 & & & $0.970^{*}$ & 0.565 \\
\hline DRG62 & 0.018 & 0.132 & & & -0.774 & -0.768 \\
\hline DRG64 & 0.001 & 0.030 & & & $2.450 * *$ & $2.559 * *$ \\
\hline DRG65 & 0.001 & 0.024 & & & $2.739 * * *$ & 0.858 \\
\hline DRG66 & 0.011 & 0.105 & & & 0.812 & 0.786 \\
\hline
\end{tabular}




\begin{tabular}{|c|c|c|c|c|c|c|}
\hline Congestive heart failure & 0.013 & 0.112 & & & & $1.814^{* * *}$ \\
\hline Valvular disease & 0.007 & 0.082 & & & & $1.021 * *$ \\
\hline $\begin{array}{l}\text { Pulmonary circulation } \\
\text { disorders }\end{array}$ & 0.003 & 0.050 & & & & $1.062 * *$ \\
\hline $\begin{array}{l}\text { Peripheral vascular } \\
\text { disorders }\end{array}$ & 0.019 & 0.138 & & & & -0.187 \\
\hline Hypertension combined & 0.099 & 0.298 & & & & 0.295 \\
\hline Paralysis & 0.016 & 0.124 & & & & $1.262^{* * *}$ \\
\hline $\begin{array}{l}\text { Other neurological } \\
\text { disorders }\end{array}$ & 0.009 & 0.092 & & & & -0.277 \\
\hline Chronic pulmonary disease & 0.012 & 0.109 & & & & $1.952 * * *$ \\
\hline $\begin{array}{l}\text { Diabetes uncomplicated } \\
\text { variation }\end{array}$ & 0.034 & 0.181 & & & & 0.165 \\
\hline Diabetes complicated & 0.045 & 0.208 & & & & $0.755^{* *}$ \\
\hline Hypothyroidism & 0.002 & 0.045 & & & & 0.902 \\
\hline Renal failure & 0.056 & 0.230 & & & & -0.106 \\
\hline Liver disease & 0.008 & 0.087 & & & & $1.379 * * *$ \\
\hline $\begin{array}{l}\text { Peptic ulcer disease } \\
\text { excluding bleeding }\end{array}$ & 0.001 & 0.025 & & & & $2.591 * * *$ \\
\hline Metastatic cancer & 0.049 & 0.216 & & & & $-1.172 *$ \\
\hline $\begin{array}{l}\text { Solid tumour without } \\
\text { metastasis variation }\end{array}$ & 0.028 & 0.165 & & & & -0.383 \\
\hline $\begin{array}{l}\text { Rheumatoid arthritis / } \\
\text { collagen vascular diseases }\end{array}$ & 0.006 & 0.077 & & & & $1.294 * *$ \\
\hline Obesity & 0.015 & 0.120 & & & & 0.580 \\
\hline Weight loss & 0.003 & 0.051 & & & & 0.779 \\
\hline Blood loss anaemia & 0.003 & 0.057 & & & & 1.065 \\
\hline Deficiency anaemias & 0.004 & 0.063 & & & & $1.051^{*}$ \\
\hline Alcohol abuse & 0.005 & 0.068 & & & & $1.257^{* * *}$ \\
\hline Drug abuse & 0.003 & 0.052 & & & & 0.333 \\
\hline Psychoses & 0.002 & 0.045 & & & & $2.318 * * *$ \\
\hline Depression & 0.005 & 0.074 & & & & 0.692 \\
\hline Constant & & & $-6.737 * * *$ & $-6.858 * * *$ & $-6.939 * * *$ & $-7.357 * * *$ \\
\hline Observations & & & 92,291 & 92,291 & 92,291 & 92,291 \\
\hline Sensitivity & & & 0.534 & 0.580 & 0.595 & 0.664 \\
\hline Specificity & & & 0.683 & 0.657 & 0.673 & 0.826 \\
\hline C-stat (Area under ROC) & & & 0.644 & 0.656 & 0.686 & 0.837 \\
\hline LR test $\mathrm{M} 1$ & & & & 7 & 29 & $262 * * *$ \\
\hline LR test M2 & & & & & 23 & $255^{* * *}$ \\
\hline LR test M3 & & & & & & $233 * * *$ \\
\hline
\end{tabular}

*** $\mathrm{p}<0.01,{ }^{*} \mathrm{p}<0.05, * \mathrm{p}<0.1$ 
Table 33: PSI12

\begin{tabular}{|c|c|c|c|c|c|c|}
\hline Variables & Mean & $\begin{array}{l}\text { Std. } \\
\text { Dev. }\end{array}$ & M1 & M2 & M3 & M4 \\
\hline PSI12 & 0.003 & 0.056 & & & & \\
\hline sex (male) & 0.483 & 0.500 & $0.255^{* * *}$ & $0.251^{* * *}$ & $0.160 * *$ & $0.277^{* * *}$ \\
\hline agecat1 $(<30)$ & 0.123 & 0.328 & $-0.978 * * *$ & $-0.969 * * *$ & $-0.687 * * *$ & $-0.301 * *$ \\
\hline agecat2 (30-39) & 0.122 & 0.327 & $-0.636 * * *$ & $-0.625 * * *$ & $-0.355 * * *$ & -0.0192 \\
\hline agecat3 (40-49) & 0.146 & 0.353 & $-0.395 * * *$ & $-0.388 * * *$ & $-0.176 * *$ & -0.103 \\
\hline agecat5 (60-69) & 0.163 & 0.370 & $0.444 * * *$ & $0.441 * * *$ & $0.203 * * *$ & 0.0699 \\
\hline agecat6 (70-79) & 0.180 & 0.384 & $0.610 * * *$ & $0.599 * * *$ & $0.292 * * *$ & $0.148^{*}$ \\
\hline agecat7 (80+) & 0.119 & 0.324 & $0.572 * * *$ & $0.551^{* * *}$ & 0.123 & 0.0377 \\
\hline agesexcat1 & 0.065 & 0.246 & -0.250 & -0.247 & $-0.300 *$ & $-0.427^{* *}$ \\
\hline agesexcat2 & 0.051 & 0.220 & -0.128 & -0.130 & -0.190 & $-0.432 * *$ \\
\hline agesexcat3 & 0.062 & 0.242 & 0.124 & 0.122 & 0.0438 & 0.00470 \\
\hline agesexcat5 & 0.086 & 0.281 & $-0.218 * *$ & $-0.218^{* *}$ & -0.107 & -0.107 \\
\hline agesexcat6 & 0.096 & 0.294 & $-0.429 * * *$ & $-0.428 * * *$ & $-0.231 * *$ & $-0.356 * * *$ \\
\hline agesexcat7 & 0.051 & 0.220 & $-0.548 * * *$ & $-0.544 * * *$ & $-0.201^{*}$ & $-0.417 * * *$ \\
\hline Maori & 0.114 & 0.318 & & -0.0442 & $-0.129 * *$ & $-0.340 * * *$ \\
\hline Pacific & 0.046 & 0.210 & & 0.0419 & 0.0288 & $-0.246 * *$ \\
\hline Asian & 0.034 & 0.181 & & $-0.444 * * *$ & $-0.406 * * *$ & $-0.454 * * *$ \\
\hline Other & 0.123 & 0.328 & & 0.0327 & 0.0482 & $0.178 * * *$ \\
\hline NZDep (lower quintile) & 0.137 & 0.344 & & $0.0999 * *$ & 0.0679 & $0.124 * *$ \\
\hline NZDep (second quintile) & 0.165 & 0.371 & & $0.0858 *$ & 0.0715 & $0.119 * *$ \\
\hline NZDep (fourth quintile) & 0.247 & 0.432 & & -0.0160 & -0.00621 & $0.0927^{*}$ \\
\hline NZDep (upper quintile) & 0.244 & 0.429 & & -0.0721 & -0.0612 & -0.0275 \\
\hline DRG1 & 0.008 & 0.088 & & & $1.417^{* * *}$ & $0.908 * * *$ \\
\hline DRG2 & 0.001 & 0.028 & & & $1.676 * * *$ & $1.829 * * *$ \\
\hline DRG3 & 0.002 & 0.049 & & & $-1.035^{*}$ & $-1.454 * *$ \\
\hline DRG4 & 0.005 & 0.070 & & & -0.410 & -0.107 \\
\hline DRG5 & 0.004 & 0.066 & & & $1.575^{* * *}$ & $0.462 * *$ \\
\hline DRG6 & 0.002 & 0.042 & & & $2.338 * * *$ & $1.131^{* * *}$ \\
\hline DRG7 & 0.001 & 0.028 & & & $1.066 * * *$ & $-2.435 * * *$ \\
\hline DRG8 & 0.004 & 0.061 & & & 0.338 & $-3.094 * * *$ \\
\hline DRG10 & 0.003 & 0.056 & & & $0.946 * * *$ & 0.0544 \\
\hline DRG11 & 0.001 & 0.022 & & & $1.551 * * *$ & $-1.796 * * *$ \\
\hline DRG12 & 0.005 & 0.072 & & & $0.502 * * *$ & -0.269 \\
\hline DRG13 & 0.007 & 0.081 & & & $1.695 * * *$ & $1.625^{* * *}$ \\
\hline DRG14 & 0.025 & 0.156 & & & $-0.693 * * *$ & $-1.248 * * *$ \\
\hline DRG15 & 0.001 & 0.037 & & & $1.809 * * *$ & $1.328 * * *$ \\
\hline DRG16 & 0.001 & 0.025 & & & 0.689 & 0.463 \\
\hline DRG17 & 0.006 & 0.076 & & & $-0.529 *$ & $-1.342 * * *$ \\
\hline DRG19 & 0.001 & 0.026 & & & 0.286 & -0.106 \\
\hline DRG20 & 0.001 & 0.034 & & & -1.354 & -1.378 \\
\hline
\end{tabular}




\begin{tabular}{|c|c|c|}
\hline DRG21 & 0.004 & 0.059 \\
\hline DRG22 & 0.002 & 0.042 \\
\hline DRG25 & 0.002 & 0.040 \\
\hline DRG32 & 0.004 & 0.065 \\
\hline DRG33 & 0.019 & 0.135 \\
\hline DRG34 & 0.006 & 0.076 \\
\hline DRG35 & 0.004 & 0.061 \\
\hline DRG36 & 0.004 & 0.066 \\
\hline DRG37 & 0.015 & 0.122 \\
\hline DRG38 & 0.010 & 0.098 \\
\hline DRG39 & 0.014 & 0.117 \\
\hline DRG40 & 0.004 & 0.060 \\
\hline DRG41 & 0.015 & 0.123 \\
\hline DRG42 & 0.004 & 0.065 \\
\hline DRG43 & 0.002 & 0.045 \\
\hline DRG44 & 0.001 & 0.034 \\
\hline DRG45 & 0.002 & 0.041 \\
\hline DRG46 & 0.021 & 0.144 \\
\hline DRG47 & 0.000 & 0.018 \\
\hline DRG48 & 0.000 & 0.015 \\
\hline DRG50 & 0.048 & 0.213 \\
\hline DRG51 & 0.015 & 0.121 \\
\hline DRG53 & 0.001 & 0.029 \\
\hline DRG54 & 0.001 & 0.035 \\
\hline DRG55 & 0.006 & 0.078 \\
\hline DRG56 & 0.013 & 0.115 \\
\hline DRG57 & 0.007 & 0.082 \\
\hline DRG58 & 0.012 & 0.107 \\
\hline DRG59 & 0.017 & 0.128 \\
\hline DRG60 & 0.007 & 0.085 \\
\hline DRG61 & 0.001 & 0.034 \\
\hline DRG64 & 0.002 & 0.042 \\
\hline DRG69 & 0.010 & 0.098 \\
\hline DRG70 & 0.013 & 0.114 \\
\hline DRG71 & 0.003 & 0.053 \\
\hline DRG72 & 0.020 & 0.141 \\
\hline DRG73 & 0.053 & 0.224 \\
\hline DRG75 & 0.001 & 0.027 \\
\hline DRG77 & 0.000 & 0.020 \\
\hline DRG78 & 0.004 & 0.063 \\
\hline DRG79 & 0.001 & 0.024 \\
\hline DRG80 & 0.001 & 0.025 \\
\hline DRG81 & 0.002 & 0.048 \\
\hline
\end{tabular}

\begin{tabular}{|c|c|}
\hline $3 * * *$ & $1.541^{* * *}$ \\
\hline $2.030 * * *$ & $1.985^{* * *}$ \\
\hline $.772 * *$ & -0.133 \\
\hline $1.257 * * *$ & $0.443 * *$ \\
\hline $1.492 * * *$ & $0.881 * * *$ \\
\hline $1.101 * * *$ & $0.844 * * *$ \\
\hline 0.156 & 0.401 \\
\hline $1.457 * * *$ & $1.000 * * *$ \\
\hline$-1.752 * * *$ & $-1.357^{* * *}$ \\
\hline$-0.502 * *$ & -0.322 \\
\hline$-2.151 * * *$ & $-1.633 * * *$ \\
\hline 0.303 & 0.444 \\
\hline$-1.218 * * *$ & $-1.114 * * *$ \\
\hline $1.346 * * *$ & $0.876 * * *$ \\
\hline $1.717 * * *$ & $1.036^{* * *}$ \\
\hline $0.859 * *$ & 0.563 \\
\hline-0.842 & -0.767 \\
\hline$-0.571 * * *$ & $-0.319 *$ \\
\hline $1.326 * * *$ & $1.021^{* *}$ \\
\hline $2.674^{* * *}$ & $1.915^{* * *}$ \\
\hline $1.524 * * *$ & $1.463^{* * *}$ \\
\hline $1.476^{* * *}$ & $1.140 * * *$ \\
\hline $1.359 * * *$ & $1.164^{* * *}$ \\
\hline $1.697^{* * *}$ & $1.493 * * *$ \\
\hline $1.682 * * *$ & $1.610 * * *$ \\
\hline$-0.889 * * *$ & $-0.668 * *$ \\
\hline 0.280 & $0.713^{* * *}$ \\
\hline-0.0366 & 0.219 \\
\hline$-1.536 * * *$ & $-1.066 * * *$ \\
\hline $0.607 * * *$ & $0.362 *$ \\
\hline $2.065^{* * *}$ & $2.066^{* * *}$ \\
\hline-1.640 & -1.173 \\
\hline-0.166 & $-0.609 * *$ \\
\hline$-2.887 * * *$ & $-2.426 * * *$ \\
\hline $1.125^{* * *}$ & $1.192^{* * *}$ \\
\hline$-0.961 * * *$ & $-0.654 * * *$ \\
\hline$-2.037 * * *$ & $-1.402 * * *$ \\
\hline $1.092 * * *$ & $1.067 * *$ \\
\hline $1.350 * * *$ & $1.167^{*}$ \\
\hline$-1.241 * *$ & -0.985 \\
\hline 0.710 & -0.156 \\
\hline $0.859 *$ & 0.559 \\
\hline $1.480 * * *$ & $0.941 * * *$ \\
\hline
\end{tabular}




\begin{tabular}{|c|c|c|c|c|}
\hline DRG82 & 0.005 & 0.072 & $0.998 * * *$ & $0.873 * * *$ \\
\hline DRG83 & 0.001 & 0.027 & -0.225 & 0.317 \\
\hline DRG84 & 0.003 & 0.059 & -0.159 & -0.214 \\
\hline DRG85 & 0.010 & 0.100 & $-0.913 * * *$ & $-0.722 * *$ \\
\hline DRG86 & 0.004 & 0.061 & $1.249 * * *$ & $1.021 * * *$ \\
\hline DRG87 & 0.002 & 0.048 & 0.272 & 0.317 \\
\hline DRG88 & 0.009 & 0.094 & $-0.890 * * *$ & -0.378 \\
\hline DRG89 & 0.001 & 0.024 & $1.284^{* * *}$ & 0.571 \\
\hline DRG90 & 0.002 & 0.045 & $1.252 * * *$ & $0.918^{* * *}$ \\
\hline DRG91 & 0.007 & 0.085 & $-3.137 * * *$ & $-2.659 * * *$ \\
\hline DRG92 & 0.001 & 0.037 & $2.123 * * *$ & $1.370 * * *$ \\
\hline DRG93 & 0.035 & 0.185 & $-0.740 * * *$ & $-0.538 * * *$ \\
\hline DRG94 & 0.020 & 0.140 & $-1.153 * * *$ & $-0.745 * * *$ \\
\hline DRG97 & 0.002 & 0.045 & $1.257^{* * *}$ & $1.215^{* * *}$ \\
\hline DRG98 & 0.000 & 0.019 & $1.086^{*}$ & 0.447 \\
\hline DRG99 & 0.002 & 0.047 & $1.547^{* * *}$ & $1.288 * * *$ \\
\hline DRG100 & 0.002 & 0.048 & $1.520 * * *$ & $0.905^{* * *}$ \\
\hline DRG101 & 0.004 & 0.059 & $1.551 * * *$ & $1.381 * * *$ \\
\hline DRG102 & 0.001 & 0.023 & $1.682 * * *$ & $0.798^{*}$ \\
\hline DRG103 & 0.001 & 0.030 & $1.806 * * *$ & $0.830 * *$ \\
\hline DRG104 & 0.006 & 0.075 & $1.471 * * *$ & $1.259 * * *$ \\
\hline DRG105 & 0.004 & 0.063 & 0.305 & $0.497^{*}$ \\
\hline DRG107 & 0.013 & 0.114 & $0.438 * * *$ & $0.450 * * *$ \\
\hline DRG109 & 0.005 & 0.070 & $1.273 * * *$ & $0.840 * * *$ \\
\hline DRG110 & 0.000 & 0.009 & $2.818^{* * *}$ & $2.838 * * *$ \\
\hline DRG111 & 0.001 & 0.024 & $3.147 * * *$ & $2.496 * * *$ \\
\hline DRG112 & 0.001 & 0.034 & $2.441 * * *$ & $2.046 * * *$ \\
\hline DRG114 & 0.000 & 0.008 & $2.486 * * *$ & -1.171 \\
\hline DRG115 & 0.000 & 0.013 & $2.293 * * *$ & -0.203 \\
\hline DRG116 & 0.001 & 0.029 & $2.326 * * *$ & $2.656^{* * *}$ \\
\hline Congestive heart failure & 0.016 & 0.124 & & $-0.482 * * *$ \\
\hline Valvular disease & 0.010 & 0.100 & & $-1.593 * * *$ \\
\hline $\begin{array}{l}\text { Pulmonary circulation } \\
\text { disorders }\end{array}$ & 0.003 & 0.054 & & $6.977 * * *$ \\
\hline $\begin{array}{l}\text { Peripheral vascular } \\
\text { disorders }\end{array}$ & 0.017 & 0.130 & & $0.302 * * *$ \\
\hline Hypertension combined & 0.103 & 0.304 & & $0.447^{* * *}$ \\
\hline Paralysis & 0.009 & 0.096 & & $0.659 * * *$ \\
\hline $\begin{array}{l}\text { Other neurological } \\
\text { disorders }\end{array}$ & 0.007 & 0.083 & & 0.188 \\
\hline Chronic pulmonary disease & 0.015 & 0.121 & & $-1.079 * * *$ \\
\hline $\begin{array}{l}\text { Diabetes uncomplicated } \\
\text { variation }\end{array}$ & 0.036 & 0.186 & & -0.0733 \\
\hline Diabetes complicated & 0.035 & 0.184 & & $-0.195 * *$ \\
\hline
\end{tabular}




\begin{tabular}{|c|c|c|c|c|c|c|}
\hline Hypothyroidism & 0.002 & 0.043 & & & & 0.187 \\
\hline Renal failure & 0.028 & 0.164 & & & & $0.509 * * *$ \\
\hline Liver disease & 0.004 & 0.065 & & & & $0.577 * * *$ \\
\hline $\begin{array}{l}\text { Peptic ulcer disease } \\
\text { excluding bleeding }\end{array}$ & 0.001 & 0.023 & & & & $0.914^{* * *}$ \\
\hline AIDS & 0.000 & 0.014 & & & & $1.574 * * *$ \\
\hline Lymphoma & 0.002 & 0.039 & & & & $1.392 * * *$ \\
\hline Metastatic cancer & 0.024 & 0.153 & & & & $1.406 * * *$ \\
\hline $\begin{array}{l}\text { Solid tumour without } \\
\text { metastasis variation }\end{array}$ & 0.013 & 0.111 & & & & $0.923 * * *$ \\
\hline $\begin{array}{l}\text { Rheumatoid arthritis / } \\
\text { collagen vascular diseases }\end{array}$ & 0.005 & 0.069 & & & & 0.0487 \\
\hline Obesity & 0.014 & 0.119 & & & & $0.223 * *$ \\
\hline Weight loss & 0.002 & 0.039 & & & & $0.951 * * *$ \\
\hline Blood loss anaemia & 0.003 & 0.059 & & & & $0.950 * * *$ \\
\hline Deficiency anaemias & 0.003 & 0.057 & & & & $0.634 * * *$ \\
\hline Alcohol abuse & 0.006 & 0.080 & & & & 0.246 \\
\hline Drug abuse & 0.003 & 0.054 & & & & $0.856 * * *$ \\
\hline Psychoses & 0.001 & 0.037 & & & & $0.689 * *$ \\
\hline Depression & 0.003 & 0.050 & & & & $1.068^{* * *}$ \\
\hline Constant & & & $-5.885 * * *$ & $-5.878 * * *$ & $-6.184 * * *$ & $-6.971 * * *$ \\
\hline Observations & & & $1,422,601$ & $1,422,601$ & $1,422,601$ & $1,422,601$ \\
\hline Sensitivity & & & 0.732 & 0.724 & 0.645 & 0.749 \\
\hline Specificity & & & 0.467 & 0.477 & 0.787 & 0.865 \\
\hline C-stat (Area under ROC) & & & 0.625 & 0.630 & 0.782 & 0.885 \\
\hline LR test M1 & & & & $39 * *$ & $4115^{* * *}$ & $24178 * * *$ \\
\hline LR test M2 & & & & & $4076^{* * *}$ & $24139 * * *$ \\
\hline LR test $M 3$ & & & & & & $20063 * * *$ \\
\hline
\end{tabular}

*** $p<0.01,{ }^{* *} p<0.05, * p<0.1$ 
Table 34: PSI13

\begin{tabular}{|c|c|c|c|c|c|c|}
\hline Variables & Mean & $\begin{array}{l}\text { Std. } \\
\text { Dev. }\end{array}$ & M1 & M2 & M3 & M4 \\
\hline PSI13 & 0.013 & 0.112 & & & & \\
\hline sex (male) & 0.545 & 0.498 & 0.220 & 0.223 & 0.322 & 0.307 \\
\hline agecat1 $(<30)$ & 0.069 & 0.253 & 0.473 & 0.414 & 0.357 & 0.403 \\
\hline agecat2 (30-39) & 0.075 & 0.263 & -0.128 & -0.161 & -0.149 & -0.142 \\
\hline agecat3 (40-49) & 0.116 & 0.320 & 0.124 & 0.110 & 0.0438 & 0.0223 \\
\hline agecat5 (60-69) & 0.206 & 0.405 & 0.463 & 0.501 & 0.312 & 0.322 \\
\hline agecat6 (70-79) & 0.240 & 0.427 & 0.427 & 0.518 & 0.366 & 0.390 \\
\hline agecat7 $(80+)$ & 0.144 & 0.351 & -0.0999 & 0.0213 & -0.345 & -0.328 \\
\hline agesexcat1 & 0.041 & 0.199 & -0.645 & -0.650 & -0.681 & -0.723 \\
\hline agesexcat2 & 0.036 & 0.187 & 0.0857 & 0.0685 & -0.106 & -0.0792 \\
\hline agesexcat3 & 0.065 & 0.246 & -0.545 & -0.559 & -0.587 & -0.516 \\
\hline agesexcat5 & 0.127 & 0.333 & -0.184 & -0.169 & -0.00357 & 0.00668 \\
\hline agesexcat6 & 0.129 & 0.335 & -0.376 & -0.372 & -0.378 & -0.376 \\
\hline agesexcat7 & 0.054 & 0.225 & 0.662 & 0.645 & 0.561 & 0.573 \\
\hline Maori & 0.115 & 0.319 & & 0.294 & 0.0915 & 0.0869 \\
\hline Pacific & 0.043 & 0.203 & & $0.567^{* *}$ & $0.789 * * *$ & $0.786 * * *$ \\
\hline Asian & 0.031 & 0.174 & & 0.264 & 0.403 & 0.387 \\
\hline Other & 0.125 & 0.331 & & $-0.377^{*}$ & -0.308 & -0.336 \\
\hline NZDep (lower quintile) & 0.132 & 0.339 & & -0.324 & -0.291 & -0.314 \\
\hline NZDep (second quintile) & 0.161 & 0.368 & & -0.154 & -0.126 & -0.134 \\
\hline NZDep (fourth quintile) & 0.243 & 0.429 & & -0.00798 & -0.0225 & -0.0327 \\
\hline NZDep (upper quintile) & 0.259 & 0.438 & & -0.0617 & -0.135 & -0.166 \\
\hline DRG1 & 0.035 & 0.183 & & & -0.393 & -0.378 \\
\hline DRG4 & 0.016 & 0.125 & & & -0.353 & -0.336 \\
\hline DRG5 & 0.003 & 0.053 & & & -0.240 & -0.324 \\
\hline DRG6 & 0.010 & 0.100 & & & $-2.091^{* *}$ & $-2.085^{* *}$ \\
\hline DRG7 & 0.049 & 0.215 & & & $-1.718^{* * *}$ & $-1.698 * * *$ \\
\hline DRG8 & 0.052 & 0.222 & & & $-1.132 * * *$ & $-1.107 * * *$ \\
\hline DRG9 & 0.005 & 0.073 & & & -1.214 & -1.167 \\
\hline DRG10 & 0.108 & 0.311 & & & $-1.872 * * *$ & $-1.845^{* * *}$ \\
\hline DRG11 & 0.026 & 0.159 & & & $-1.187^{* *}$ & $-1.258 * *$ \\
\hline DRG12 & 0.010 & 0.099 & & & $0.708^{*}$ & $0.695^{*}$ \\
\hline DRG17 & 0.019 & 0.135 & & & $1.139 * * *$ & $1.084^{* * *}$ \\
\hline DRG19 & 0.004 & 0.060 & & & 0.469 & 0.204 \\
\hline DRG21 & 0.004 & 0.061 & & & -0.0198 & -0.121 \\
\hline DRG22 & 0.004 & 0.061 & & & $1.452 * * *$ & $1.433 * * *$ \\
\hline DRG24 & 0.041 & 0.199 & & & $-1.324 * *$ & $-1.316 * *$ \\
\hline DRG25 & 0.018 & 0.133 & & & -1.530 & -1.538 \\
\hline DRG26 & 0.006 & 0.076 & & & -0.252 & -0.262 \\
\hline DRG30 & 0.010 & 0.101 & & & -0.412 & -0.487 \\
\hline
\end{tabular}




\begin{tabular}{|c|c|c|c|c|c|c|}
\hline DRG33 & 0.011 & 0.105 & & & -0.231 & -0.182 \\
\hline DRG34 & 0.004 & 0.062 & & & -0.257 & -0.271 \\
\hline DRG35 & 0.022 & 0.148 & & & -0.887 & -0.872 \\
\hline DRG36 & 0.013 & 0.112 & & & 0.469 & 0.475 \\
\hline DRG37 & 0.034 & 0.180 & & & $0.589 * *$ & $0.551 * *$ \\
\hline Congestive heart failure & 0.067 & 0.251 & & & $1.144 * * *$ & $1.125^{* * *}$ \\
\hline Valvular disease & 0.058 & 0.233 & & & $0.633^{* * *}$ & $0.623 * * *$ \\
\hline $\begin{array}{l}\text { Pulmonary circulation } \\
\text { disorders }\end{array}$ & 0.018 & 0.131 & & & $1.051^{* * *}$ & $1.046^{* * *}$ \\
\hline $\begin{array}{l}\text { Peripheral vascular } \\
\text { disorders }\end{array}$ & 0.073 & 0.259 & & & $0.410^{*}$ & $0.408^{*}$ \\
\hline Hypertension combined & 0.292 & 0.454 & & & 0.121 & 0.132 \\
\hline Paralysis & 0.037 & 0.188 & & & $0.823 * * *$ & $0.841^{* * *}$ \\
\hline $\begin{array}{l}\text { Other neurological } \\
\text { disorders }\end{array}$ & 0.019 & 0.137 & & & -0.153 & -0.185 \\
\hline Chronic pulmonary disease & 0.046 & 0.209 & & & $0.515 * *$ & $0.496 * *$ \\
\hline $\begin{array}{l}\text { Diabetes uncomplicated } \\
\text { variation }\end{array}$ & 0.063 & 0.244 & & & 0.177 & 0.182 \\
\hline Diabetes complicated & 0.068 & 0.251 & & & 0.0524 & 0.0880 \\
\hline Hypothyroidism & 0.007 & 0.081 & & & 0.704 & 0.624 \\
\hline Renal failure & 0.003 & 0.050 & & & 1.021 & 1.046 \\
\hline Liver disease & 0.009 & 0.093 & & & $1.454 * * *$ & $1.280 * * *$ \\
\hline $\begin{array}{l}\text { Peptic ulcer disease } \\
\text { excluding bleeding }\end{array}$ & 0.002 & 0.040 & & & 0.212 & 0.102 \\
\hline $\begin{array}{l}\text { Rheumatoid arthritis / } \\
\text { collagen vascular diseases }\end{array}$ & 0.012 & 0.108 & & & & 0.465 \\
\hline Obesity & 0.032 & 0.175 & & & & -0.0892 \\
\hline Weight loss & 0.005 & 0.069 & & & & $1.479 * * *$ \\
\hline Blood loss anaemia & 0.006 & 0.078 & & & & 0.621 \\
\hline Deficiency anaemias & 0.010 & 0.100 & & & & 0.179 \\
\hline Alcohol abuse & 0.011 & 0.103 & & & & $0.686^{*}$ \\
\hline Psychoses & 0.005 & 0.070 & & & & 0.751 \\
\hline Depression & 0.014 & 0.116 & & & & -1.157 \\
\hline Constant & & & $-4.623 * * *$ & $-4.624 * * *$ & $-4.751 * * *$ & $-4.778 * * *$ \\
\hline Observations & & & 21,329 & 21,329 & 21,329 & 21,329 \\
\hline Sensitivity & & & 0.502 & 0.597 & 0.678 & 0.685 \\
\hline Specificity & & & 0.604 & 0.538 & 0.730 & 0.735 \\
\hline C-stat (Area under ROC) & & & 0.572 & 0.591 & 0.769 & 0.776 \\
\hline LR test $\mathrm{M} 1$ & & & & $14^{*}$ & $280 * * *$ & $300 * * *$ \\
\hline LR test M2 & & & & & $266^{* * *}$ & $286 * * *$ \\
\hline LR test M3 & & & & & & $20 * *$ \\
\hline
\end{tabular}

*** $p<0.01,{ }^{* *} p<0.05, * p<0.1$ 
Table 35: PSI14

\begin{tabular}{|c|c|c|c|c|c|c|}
\hline Variables & Mean & $\begin{array}{l}\text { Std. } \\
\text { Dev. }\end{array}$ & M1 & M2 & M3 & M4 \\
\hline PSI14 & 0.005 & 0.068 & & & & \\
\hline sex (male) & 0.429 & 0.495 & 0.242 & 0.249 & 0.301 & 0.333 \\
\hline agecat1 $(<30)$ & 0.087 & 0.282 & $-1.430 * * *$ & $-1.505^{* * *}$ & $-1.435 * * *$ & $-1.254 * * *$ \\
\hline agecat2 (30-39) & 0.114 & 0.317 & $-0.924 * * *$ & $-0.978 * * *$ & $-0.949 * * *$ & $-0.846 * * *$ \\
\hline agecat3 (40-49) & 0.172 & 0.377 & $-0.710 * * *$ & $-0.748 * * *$ & $-0.681 * * *$ & $-0.639 * * *$ \\
\hline agecat5 (60-69) & 0.183 & 0.387 & 0.123 & 0.150 & 0.142 & 0.0681 \\
\hline agecat6 (70-79) & 0.192 & 0.394 & $0.395^{* *}$ & $0.448 * *$ & $0.450 * *$ & $0.314^{*}$ \\
\hline agecat7 $(80+)$ & 0.099 & 0.299 & -0.158 & -0.0853 & -0.0474 & -0.210 \\
\hline agesexcat1 & 0.044 & 0.206 & 0.331 & 0.356 & 0.376 & 0.404 \\
\hline agesexcat2 & 0.036 & 0.186 & 0.0414 & 0.0587 & 0.0776 & 0.0991 \\
\hline agesexcat3 & 0.047 & 0.211 & 0.0585 & 0.0692 & 0.0174 & 0.0267 \\
\hline agesexcat5 & 0.096 & 0.295 & 0.0660 & 0.0609 & 0.0728 & 0.103 \\
\hline agesexcat6 & 0.100 & 0.300 & -0.212 & -0.214 & -0.217 & -0.206 \\
\hline agesexcat7 & 0.043 & 0.202 & 0.493 & 0.489 & 0.451 & 0.424 \\
\hline Maori & 0.110 & 0.313 & & $0.421 * * *$ & $0.439 * * *$ & $0.292 * *$ \\
\hline Pacific & 0.043 & 0.203 & & 0.330 & $0.355^{*}$ & 0.199 \\
\hline Asian & 0.036 & 0.186 & & -0.00875 & 0.0204 & 0.0517 \\
\hline Other & 0.122 & 0.327 & & -0.0444 & -0.0477 & -0.0366 \\
\hline NZDep (lower quintile) & 0.141 & 0.348 & & -0.0706 & -0.0919 & -0.0619 \\
\hline NZDep (second quintile) & 0.172 & 0.378 & & -0.188 & -0.190 & -0.161 \\
\hline NZDep (fourth quintile) & 0.245 & 0.430 & & 0.0508 & 0.0312 & 0.0189 \\
\hline NZDep (upper quintile) & 0.234 & 0.423 & & 0.0541 & 0.0369 & 0.0172 \\
\hline DRG1 & 0.027 & 0.162 & & & 0.0386 & -0.155 \\
\hline DRG2 & 0.198 & 0.399 & & & $0.345 * * *$ & $0.277^{* *}$ \\
\hline DRG3 & 0.048 & 0.214 & & & 0.185 & 0.198 \\
\hline DRG4 & 0.044 & 0.205 & & & 0.0218 & -0.0544 \\
\hline DRG5 & 0.029 & 0.168 & & & -0.276 & -0.187 \\
\hline DRG6 & 0.018 & 0.133 & & & $0.755^{* * *}$ & $0.735 * * *$ \\
\hline DRG7 & 0.018 & 0.133 & & & $0.759 * * *$ & $0.687 * * *$ \\
\hline DRG8 & 0.166 & 0.372 & & & 0.0737 & 0.182 \\
\hline DRG9 & 0.008 & 0.090 & & & $1.314 * * *$ & $1.178 * * *$ \\
\hline DRG10 & 0.012 & 0.111 & & & $2.497 * * *$ & $2.502 * * *$ \\
\hline Congestive heart failure & 0.026 & 0.159 & & & & $0.739 * * *$ \\
\hline Valvular disease & 0.011 & 0.103 & & & & 0.199 \\
\hline $\begin{array}{l}\text { Pulmonary circulation } \\
\text { disorders }\end{array}$ & 0.005 & 0.074 & & & & -0.0499 \\
\hline $\begin{array}{l}\text { Peripheral vascular } \\
\text { disorders }\end{array}$ & 0.018 & 0.134 & & & & 0.351 \\
\hline Hypertension combined & 0.122 & 0.328 & & & & $0.242^{* *}$ \\
\hline Paralysis & 0.010 & 0.098 & & & & 0.389 \\
\hline Other neurological & 0.009 & 0.093 & & & & 0.244 \\
\hline
\end{tabular}




\begin{tabular}{|c|c|c|c|c|c|c|}
\hline Chronic pulmonary disease & 0.030 & 0.170 & & & & $0.789 * * *$ \\
\hline $\begin{array}{l}\text { Diabetes uncomplicated } \\
\text { variation }\end{array}$ & 0.051 & 0.220 & & & & 0.189 \\
\hline Diabetes complicated & 0.026 & 0.159 & & & & -0.0797 \\
\hline Hypothyroidism & 0.003 & 0.052 & & & & 0.117 \\
\hline Renal failure & 0.002 & 0.044 & & & & 0.164 \\
\hline Liver disease & 0.011 & 0.104 & & & & 0.0183 \\
\hline $\begin{array}{l}\text { Peptic ulcer disease } \\
\text { excluding bleeding }\end{array}$ & 0.002 & 0.047 & & & & -0.764 \\
\hline Lymphoma & 0.000 & 0.000 & & & & -0.592 \\
\hline Metastatic cancer & 0.003 & 0.050 & & & & $0.309 * *$ \\
\hline $\begin{array}{l}\text { Solid tumour without } \\
\text { metastasis variation }\end{array}$ & 0.099 & 0.298 & & & & 0.176 \\
\hline $\begin{array}{l}\text { Rheumatoid arthritis / } \\
\text { collagen vascular diseases }\end{array}$ & 0.028 & 0.164 & & & & 0.598 \\
\hline Obesity & 0.004 & 0.066 & & & & $1.084^{* * *}$ \\
\hline Weight loss & 0.026 & 0.160 & & & & $1.417 * * *$ \\
\hline Blood loss anaemia & 0.003 & 0.058 & & & & 0.446 \\
\hline Deficiency anaemias & 0.011 & 0.105 & & & & $0.622 * *$ \\
\hline Alcohol abuse & 0.008 & 0.090 & & & & -0.109 \\
\hline Drug abuse & 0.008 & 0.087 & & & & 0.367 \\
\hline Psychoses & 0.003 & 0.056 & & & & 0.171 \\
\hline Depression & 0.002 & 0.049 & & & & $1.147 * * *$ \\
\hline Constant & 0.004 & 0.067 & $-5.357 * * *$ & $-5.419 * * *$ & $-5.712 * * *$ & $-5.948 * * *$ \\
\hline Observations & & & 123,952 & 123,952 & 123,952 & 123,952 \\
\hline Sensitivity & & & 0.691 & 0.692 & 0.649 & 0.608 \\
\hline Specificity & & & 0.520 & 0.531 & 0.603 & 0.713 \\
\hline C-stat (Area under ROC) & & & 0.634 & 0.644 & 0.688 & 0.731 \\
\hline LR test M1 & & & & $18^{* *}$ & $220 * * *$ & $378 * * *$ \\
\hline LR test $M 2$ & & & & & $202 * * *$ & $359 * * *$ \\
\hline LR test M3 & & & & & & $158^{* * *}$ \\
\hline
\end{tabular}

*** $p<0.01, * * p<0.05, * p<0.1$ 
Table 36: PSI15

\begin{tabular}{|c|c|c|c|c|c|c|}
\hline Variables & Mean & $\begin{array}{l}\text { Std. } \\
\text { Dev. }\end{array}$ & M1 & M2 & M3 & M4 \\
\hline PSI15 & 0.002 & 0.047 & & & & \\
\hline sex (male) & 0.489 & 0.500 & $-0.410 * * *$ & $-0.409 * * *$ & $-0.188 * * *$ & $-0.182 * * *$ \\
\hline agecat1 $(<30)$ & 0.099 & 0.299 & $-0.496 * * *$ & $-0.481 * * *$ & $-0.412 * * *$ & $-0.404 * * *$ \\
\hline agecat2 (30-39) & 0.099 & 0.299 & $0.166^{* * *}$ & $0.172 * * *$ & 0.0385 & 0.0442 \\
\hline agecat3 (40-49) & 0.127 & 0.333 & $0.162 * * *$ & $0.165 * * *$ & 0.000613 & 0.000386 \\
\hline agecat5 (60-69) & 0.168 & 0.374 & 0.0742 & 0.0662 & $0.112^{* *}$ & $0.107^{* *}$ \\
\hline agecat6 (70-79) & 0.195 & 0.396 & $0.108 * *$ & $0.0778 *$ & $0.253^{* * *}$ & $0.235^{* * *}$ \\
\hline agecat7 (80+) & 0.165 & 0.371 & $-0.376 * * *$ & $-0.422 * * *$ & $0.0925^{*}$ & 0.0584 \\
\hline agesexcat1 & 0.048 & 0.214 & 0.107 & 0.109 & $0.312 * * *$ & $0.317 * * *$ \\
\hline agesexcat2 & 0.045 & 0.206 & $-0.588 * * *$ & $-0.587^{* * *}$ & $-0.243^{* * *}$ & $-0.240 * *$ \\
\hline agesexcat3 & 0.060 & 0.238 & $-0.313^{* * *}$ & $-0.311^{* * *}$ & -0.0412 & -0.0371 \\
\hline agesexcat5 & 0.090 & 0.286 & $0.218 * * *$ & $0.215^{* * *}$ & 0.0548 & 0.0570 \\
\hline agesexcat6 & 0.103 & 0.304 & $0.268 * * *$ & $0.265^{* * *}$ & 0.0479 & 0.0509 \\
\hline agesexcat7 & 0.068 & 0.252 & $0.317^{* * *}$ & $0.316 * * *$ & 0.0404 & 0.0417 \\
\hline Maori & 0.133 & 0.340 & & $-0.203^{* * *}$ & 0.0199 & 0.0148 \\
\hline Pacific & 0.048 & 0.213 & & $-0.161^{* * *}$ & 0.0471 & 0.0239 \\
\hline Asian & 0.034 & 0.180 & & $0.0983^{*}$ & $0.181^{* * *}$ & $0.181^{* * *}$ \\
\hline Other & 0.121 & 0.326 & & 0.0244 & 0.0320 & 0.0325 \\
\hline NZDep (lower quintile) & 0.134 & 0.341 & & -0.0107 & -0.00463 & -0.00126 \\
\hline NZDep (second quintile) & 0.160 & 0.367 & & 0.0445 & 0.0275 & 0.0307 \\
\hline NZDep (fourth quintile) & 0.245 & 0.430 & & 0.0104 & 0.0209 & 0.0188 \\
\hline NZDep (upper quintile) & 0.258 & 0.438 & & $-0.137 * * *$ & $-0.0694 * *$ & $-0.0729 * *$ \\
\hline DRG1 & 0.002 & 0.049 & & & $1.951 * * *$ & $1.920 * * *$ \\
\hline DRG2 & 0.000 & 0.016 & & & $2.507^{* * *}$ & $2.482 * * *$ \\
\hline DRG3 & 0.001 & 0.027 & & & $1.604^{* * *}$ & $1.507^{* * *}$ \\
\hline DRG4 & 0.002 & 0.039 & & & -0.648 & -0.641 \\
\hline DRG6 & 0.000 & 0.012 & & & $2.817^{* * *}$ & $2.865^{* * *}$ \\
\hline DRG7 & 0.002 & 0.043 & & & $1.236 * * *$ & $1.228 * * *$ \\
\hline DRG8 & 0.001 & 0.023 & & & $2.179 * * *$ & $2.186 * * *$ \\
\hline DRG9 & 0.001 & 0.037 & & & $2.363^{* * *}$ & $2.329 * * *$ \\
\hline DRG10 & 0.001 & 0.024 & & & $1.806 * * *$ & $1.809 * * *$ \\
\hline DRG11 & 0.019 & 0.135 & & & $-1.512 * * *$ & $-1.566 * * *$ \\
\hline DRG12 & 0.020 & 0.139 & & & $-2.779 * * *$ & $-2.848 * * *$ \\
\hline DRG14 & 0.001 & 0.036 & & & 0.280 & 0.224 \\
\hline DRG15 & 0.000 & 0.015 & & & $1.142^{* *}$ & $1.033^{*}$ \\
\hline DRG16 & 0.000 & 0.016 & & & $2.943 * * *$ & $2.680 * * *$ \\
\hline DRG17 & 0.001 & 0.034 & & & $3.008 * * *$ & $2.826 * * *$ \\
\hline DRG19 & 0.001 & 0.031 & & & $2.495 * * *$ & $2.369 * * *$ \\
\hline DRG20 & 0.000 & 0.013 & & & $3.277^{* * *}$ & $3.107^{* * *}$ \\
\hline DRG21 & 0.002 & 0.040 & & & $2.161^{* * *}$ & $2.042 * * *$ \\
\hline
\end{tabular}




\begin{tabular}{|c|c|c|c|c|}
\hline DRG22 & 0.002 & 0.045 & $3.067 * * *$ & $2.882 * * *$ \\
\hline DRG23 & 0.009 & 0.093 & $1.247 * * *$ & $1.192 * * *$ \\
\hline DRG24 & 0.000 & 0.021 & 0.670 & 0.436 \\
\hline DRG25 & 0.002 & 0.044 & $0.857 * * *$ & $0.810 * * *$ \\
\hline DRG27 & 0.001 & 0.034 & $0.614^{*}$ & $0.602 *$ \\
\hline DRG28 & 0.001 & 0.023 & $1.383 * * *$ & $1.327 * * *$ \\
\hline DRG29 & 0.006 & 0.080 & -0.239 & $-0.347^{*}$ \\
\hline DRG30 & 0.010 & 0.099 & $-0.374 * *$ & $-0.452 * *$ \\
\hline DRG31 & 0.013 & 0.111 & $-2.493 * * *$ & $-2.599 * * *$ \\
\hline DRG32 & 0.005 & 0.067 & $-0.522 *$ & $-0.642 * *$ \\
\hline DRG33 & 0.002 & 0.040 & $-1.302 *$ & $-1.357^{*}$ \\
\hline DRG34 & 0.005 & 0.068 & $-1.261 * * *$ & $-1.305^{* * *}$ \\
\hline DRG42 & 0.001 & 0.036 & $3.514 * * *$ & $3.529 * * *$ \\
\hline DRG43 & 0.006 & 0.076 & $4.076 * * *$ & $4.082 * * *$ \\
\hline DRG44 & 0.002 & 0.043 & $3.003 * * *$ & $2.977^{* * *}$ \\
\hline DRG45 & 0.001 & 0.034 & $0.922 * * *$ & $0.913 * * *$ \\
\hline DRG46 & 0.001 & 0.037 & $3.728 * * *$ & $3.700 * * *$ \\
\hline DRG47 & 0.005 & 0.069 & -0.416 & -0.425 \\
\hline DRG48 & 0.003 & 0.055 & $0.849 * * *$ & $0.828 * * *$ \\
\hline DRG49 & 0.004 & 0.066 & $1.083 * * *$ & $1.079 * * *$ \\
\hline DRG50 & 0.001 & 0.034 & $1.666^{* * *}$ & $1.650 * * *$ \\
\hline DRG51 & 0.005 & 0.069 & $1.305^{* * *}$ & $1.292 * * *$ \\
\hline DRG52 & 0.001 & 0.037 & $1.889 * * *$ & $1.884^{* * *}$ \\
\hline DRG53 & 0.003 & 0.058 & -0.146 & -0.0731 \\
\hline DRG54 & 0.003 & 0.056 & $-2.011 * * *$ & $-2.054 * * *$ \\
\hline DRG55 & 0.004 & 0.063 & $-1.544 * * *$ & $-1.548 * * *$ \\
\hline DRG56 & 0.023 & 0.149 & $-2.437 * * *$ & $-2.435^{* * *}$ \\
\hline DRG57 & 0.005 & 0.070 & $-1.144 * * *$ & $-1.157 * * *$ \\
\hline DRG58 & 0.001 & 0.025 & $3.920 * * *$ & $3.857 * * *$ \\
\hline DRG59 & 0.000 & 0.019 & $4.082 * * *$ & $4.068 * * *$ \\
\hline DRG60 & 0.001 & 0.023 & $3.414 * * *$ & $3.393 * * *$ \\
\hline DRG61 & 0.007 & 0.081 & $3.026 * * *$ & $3.010^{* * *}$ \\
\hline DRG62 & 0.000 & 0.010 & $1.419 * *$ & $1.441 * *$ \\
\hline DRG63 & 0.000 & 0.011 & $2.238 * * *$ & $2.195^{* * *}$ \\
\hline DRG64 & 0.000 & 0.008 & $2.751 * * *$ & $2.749 * * *$ \\
\hline DRG66 & 0.002 & 0.045 & -0.484 & -0.453 \\
\hline DRG67 & 0.002 & 0.049 & $-1.673 * *$ & $-1.662 * *$ \\
\hline DRG68 & 0.006 & 0.077 & $-0.814 * * *$ & $-0.835 * * *$ \\
\hline DRG70 & 0.015 & 0.121 & $0.238 * *$ & $0.223^{* *}$ \\
\hline DRG71 & 0.005 & 0.068 & -0.146 & -0.159 \\
\hline DRG72 & 0.000 & 0.020 & $1.994 * * *$ & $1.971 * * *$ \\
\hline DRG73 & 0.002 & 0.044 & $1.843 * * *$ & $1.842^{* * *}$ \\
\hline DRG74 & 0.004 & 0.060 & $0.562 * * *$ & $0.561^{* * *}$ \\
\hline
\end{tabular}




\begin{tabular}{|c|c|c|c|c|}
\hline DRG75 & 0.005 & 0.072 & 0.171 & 0.166 \\
\hline DRG76 & 0.002 & 0.048 & 0.175 & 0.165 \\
\hline DRG77 & 0.011 & 0.103 & $-1.778 * * *$ & $-1.778 * * *$ \\
\hline DRG80 & 0.001 & 0.023 & 0.735 & 0.736 \\
\hline DRG84 & 0.001 & 0.030 & $0.683^{*}$ & $0.644 *$ \\
\hline DRG85 & 0.018 & 0.131 & $-0.951 * * *$ & $-0.943 * * *$ \\
\hline DRG86 & 0.000 & 0.015 & $3.582^{* * *}$ & $3.555^{* * *}$ \\
\hline DRG87 & 0.000 & 0.011 & $3.018^{* * *}$ & $2.892^{* * *}$ \\
\hline DRG88 & 0.001 & 0.035 & $1.602^{* * *}$ & $1.597 * * *$ \\
\hline DRG89 & 0.000 & 0.014 & $1.905^{* * *}$ & $1.651 * * *$ \\
\hline DRG90 & 0.005 & 0.069 & $-1.508 * * *$ & $-1.505^{* * *}$ \\
\hline DRG91 & 0.000 & 0.014 & $3.296 * * *$ & $3.238 * * *$ \\
\hline DRG92 & 0.001 & 0.027 & $4.175^{* * *}$ & $4.158^{* * *}$ \\
\hline DRG93 & 0.002 & 0.040 & $2.974 * * *$ & $2.980 * * *$ \\
\hline DRG94 & 0.001 & 0.033 & $2.380 * * *$ & $2.373 * * *$ \\
\hline DRG95 & 0.003 & 0.056 & $1.687^{* * *}$ & $1.677^{* * *}$ \\
\hline DRG96 & 0.001 & 0.034 & $1.116^{* * *}$ & $1.113^{* * *}$ \\
\hline DRG97 & 0.003 & 0.052 & -0.335 & -0.359 \\
\hline DRG98 & 0.011 & 0.103 & $-1.933 * * *$ & $-1.924 * * *$ \\
\hline DRG99 & 0.007 & 0.081 & $0.603 * * *$ & $0.599 * * *$ \\
\hline DRG100 & 0.009 & 0.093 & $-0.384 * *$ & $-0.378 *$ \\
\hline DRG101 & 0.001 & 0.027 & $3.093 * * *$ & $3.090 * * *$ \\
\hline DRG102 & 0.003 & 0.053 & $1.528 * * *$ & $1.533 * * *$ \\
\hline DRG103 & 0.000 & 0.013 & $3.343 * * *$ & $3.350 * * *$ \\
\hline DRG104 & 0.001 & 0.025 & $2.922^{* * *}$ & $2.894 * * *$ \\
\hline DRG105 & 0.002 & 0.048 & $2.635^{* * *}$ & $2.633^{* * *}$ \\
\hline DRG106 & 0.000 & 0.021 & $3.402 * * *$ & $3.442 * * *$ \\
\hline DRG107 & 0.011 & 0.105 & $2.694 * * *$ & $2.679 * * *$ \\
\hline DRG108 & 0.006 & 0.079 & $2.000 * * *$ & $1.997^{* * *}$ \\
\hline DRG111 & 0.001 & 0.025 & $3.152^{* * *}$ & $3.151^{* * *}$ \\
\hline DRG112 & 0.000 & 0.011 & $3.105^{* * *}$ & $3.090 * * *$ \\
\hline DRG113 & 0.001 & 0.026 & $2.073^{* * *}$ & $2.052 * * *$ \\
\hline DRG114 & 0.001 & 0.027 & $2.528^{* * *}$ & $2.483 * * *$ \\
\hline DRG115 & 0.001 & 0.033 & $0.950 * * *$ & $0.954 * * *$ \\
\hline DRG116 & 0.000 & 0.013 & $3.571 * * *$ & $3.630 * * *$ \\
\hline DRG117 & 0.000 & 0.017 & $1.672 * * *$ & $1.693 * * *$ \\
\hline DRG118 & 0.002 & 0.042 & $2.000 * * *$ & $1.965^{* * *}$ \\
\hline DRG119 & 0.004 & 0.063 & $-0.969 * *$ & $-0.956 * *$ \\
\hline DRG120 & 0.004 & 0.063 & $-1.078 * * *$ & $-1.090 * * *$ \\
\hline DRG122 & 0.004 & 0.063 & $1.573^{* * *}$ & $1.569 * * *$ \\
\hline DRG123 & 0.004 & 0.066 & $-0.480 *$ & $-0.492 *$ \\
\hline DRG124 & 0.002 & 0.039 & $1.743 * * *$ & $1.730 * * *$ \\
\hline DRG125 & 0.033 & 0.178 & -0.102 & $-0.145^{*}$ \\
\hline
\end{tabular}




\begin{tabular}{|c|c|c|c|c|c|c|}
\hline DRG126 & 0.000 & 0.019 & & & $2.372 * * *$ & $2.370 * * *$ \\
\hline DRG127 & 0.000 & 0.008 & & & $4.480 * * *$ & $4.061 * * *$ \\
\hline Congestive heart failure & 0.037 & 0.188 & & & & $0.116 * *$ \\
\hline Valvular disease & 0.017 & 0.131 & & & & $0.179 * *$ \\
\hline $\begin{array}{l}\text { Pulmonary circulation } \\
\text { disorders }\end{array}$ & 0.005 & 0.072 & & & & $0.369 * * *$ \\
\hline $\begin{array}{l}\text { Peripheral vascular } \\
\text { disorders }\end{array}$ & 0.018 & 0.133 & & & & $0.337 * * *$ \\
\hline Hypertension combined & 0.135 & 0.342 & & & & $0.110 * * *$ \\
\hline Paralysis & 0.026 & 0.160 & & & & 0.0255 \\
\hline $\begin{array}{l}\text { Other neurological } \\
\text { disorders }\end{array}$ & 0.021 & 0.143 & & & & $-0.308 * * *$ \\
\hline Chronic pulmonary disease & 0.030 & 0.170 & & & & $0.254 * * *$ \\
\hline $\begin{array}{l}\text { Diabetes uncomplicated } \\
\text { variation }\end{array}$ & 0.046 & 0.209 & & & & $-0.174 * * *$ \\
\hline Diabetes complicated & 0.051 & 0.219 & & & & -0.0607 \\
\hline Hypothyroidism & 0.004 & 0.061 & & & & 0.170 \\
\hline Renal failure & 0.076 & 0.264 & & & & $-0.157 * * *$ \\
\hline Liver disease & 0.008 & 0.089 & & & & $0.379 * * *$ \\
\hline $\begin{array}{l}\text { Peptic ulcer disease } \\
\text { excluding bleeding }\end{array}$ & 0.001 & 0.035 & & & & $0.568 * * *$ \\
\hline AIDS & 0.000 & 0.019 & & & & -0.720 \\
\hline Lymphoma & 0.008 & 0.088 & & & & $-0.881 * * *$ \\
\hline Metastatic cancer & 0.030 & 0.170 & & & & $-0.214 * * *$ \\
\hline $\begin{array}{l}\text { Solid tumour without } \\
\text { metastasis variation }\end{array}$ & 0.019 & 0.135 & & & & $0.141^{* *}$ \\
\hline $\begin{array}{l}\text { Rheumatoid arthritis / } \\
\text { collagen vascular diseases }\end{array}$ & 0.008 & 0.088 & & & & -0.209 \\
\hline Obesity & 0.018 & 0.131 & & & & $0.291 * * *$ \\
\hline Weight loss & 0.004 & 0.065 & & & & $0.558 * * *$ \\
\hline Blood loss anaemia & 0.004 & 0.067 & & & & $0.291 * * *$ \\
\hline Deficiency anaemias & 0.007 & 0.082 & & & & -0.0181 \\
\hline Alcohol abuse & 0.015 & 0.122 & & & & $-0.409 * * *$ \\
\hline Drug abuse & 0.006 & 0.078 & & & & -0.0615 \\
\hline Psychoses & 0.003 & 0.056 & & & & -0.314 \\
\hline Depression & 0.009 & 0.094 & & & & 0.0150 \\
\hline Constant & & & $-5.936 * * *$ & $-5.875^{* * *}$ & $-6.902 * * *$ & $-6.901 * * *$ \\
\hline Observations & & & $4,536,793$ & $4,536,793$ & $4,536,793$ & $4,536,793$ \\
\hline Sensitivity & & & 0.685 & 0.636 & 0.657 & 0.658 \\
\hline Specificity & & & 0.445 & 0.505 & 0.895 & 0.894 \\
\hline C-stat (Area under ROC) & & & 0.581 & 0.587 & 0.836 & 0.843 \\
\hline LR test M1 & & & & $123^{* * *}$ & $23615^{* * *}$ & $23875 * * *$ \\
\hline LR test $M 2$ & & & & & $23493 * * *$ & $23753 * * *$ \\
\hline LR test $\mathrm{M} 3$ & & & & & & $260 * * *$ \\
\hline
\end{tabular}


Table 37: PSI17

\begin{tabular}{lllll}
\hline Variables & Mean & Std. Dev. & M1 & M2 \\
\hline PSI17 & 0.015 & 0.121 & & \\
sex (male) & 0.513 & 0.500 & $0.309^{* * *}$ & $0.309^{* * *}$ \\
Maori & 0.209 & 0.406 & & $-0.366^{* * *}$ \\
Pacific & 0.109 & 0.312 & & $0.497^{* * *}$ \\
Asian & 0.088 & 0.283 & & $0.960^{* * *}$ \\
Other & 0.085 & 0.279 & & $0.416^{* * *}$ \\
NZDep (lower quintile) & 0.150 & 0.357 & & 0.00610 \\
NZDep (second quintile) & 0.160 & 0.367 & & $0.0862^{* *}$ \\
NZDep (fourth quintile) & 0.183 & 0.387 & & -0.0376 \\
NZDep (upper quintile) & 0.220 & 0.414 & & $-0.134^{* * *}$ \\
Constant & 0.286 & 0.452 & $-4.356^{* * *}$ & $-4.500^{* * *}$ \\
Observations & & & 491,994 & 491,994 \\
\hline Sensitivity & & & 0.588 & 0.552 \\
Specificity & & & 0.488 & 0.623 \\
C-stat (Area under ROC) & & & 0.538 & 0.622 \\
LR test M1 & & & $1266^{* * *}$ \\
\hline
\end{tabular}

${ }^{* * *} p<0.01, * * p<0.05, * p<0.1$ 
Table 38: PSI18

\begin{tabular}{lllll}
\hline Variables & Mean & Std. Dev. & M1 & M2 \\
\hline PSI18 & 0.063 & 0.242 & & \\
agecat1 (<30) & 0.476 & 0.499 & $0.115^{* * *}$ & $0.121^{* * *}$ \\
agecat3 (40-49) & 0.029 & 0.168 & $-0.703^{* * *}$ & $-0.708^{* * *}$ \\
agecat4 (50-59) & 0.000 & 0.007 & $2.747^{*}$ & $2.666^{*}$ \\
Maori & 0.108 & 0.311 & & $-0.360^{* * *}$ \\
Pacific & 0.053 & 0.225 & & -0.114 \\
Asian & 0.133 & 0.339 & & $0.464^{* * *}$ \\
Other & 0.125 & 0.331 & & -0.0368 \\
NZDep (lower quintile) & 0.193 & 0.395 & & $-0.125^{* *}$ \\
NZDep (second quintile) & 0.193 & 0.394 & & -0.0314 \\
NZDep (fourth quintile) & 0.199 & 0.399 & & -0.0556 \\
NZDep (upper quintile) & 0.209 & 0.407 & & $0.155^{* *}$ \\
Constant & 0.204 & 0.403 & $-2.747^{* * *}$ & $-2.773^{* * *}$ \\
Observations & & & 46,002 & 46,002 \\
\hline Sensitivity & & & 0.510 & 0.443 \\
Specificity & & & 0.526 & 0.645 \\
C-stat (Area under ROC) & & & & 0.566 \\
LR test M1 & & & & $144^{* * *}$ \\
\hline
\end{tabular}

*** $p<0.01, * * p<0.05, * p<0.1$ 
Table 39: PSI19

\begin{tabular}{lllll}
\hline Variables & Mean & Std. Dev. & M1 & M2 \\
\hline PSI19 & 0.014 & 0.119 & & \\
agecat1 (<30) & 0.545 & 0.498 & $0.0989 * *$ & $0.214^{* * *}$ \\
agecat3 (40-49) & 0.029 & 0.168 & $-0.759^{* * *}$ & $-0.732^{* * *}$ \\
Maori & 0.236 & 0.425 & & $-0.522^{* * *}$ \\
Pacific & 0.126 & 0.332 & & $-0.174^{* * *}$ \\
Asian & 0.077 & 0.267 & & $0.729 * * *$ \\
Other & 0.088 & 0.283 & & 0.06 \\
NZDep (lower quintile) & 0.134 & 0.340 & & 0.018 \\
NZDep (second quintile) & 0.147 & 0.354 & & -0.0343 \\
NZDep (fourth quintile) & 0.224 & 0.417 & & -0.0038 \\
NZDep (upper quintile) & 0.316 & 0.465 & & -0.0708 \\
Constant & & & $-4.269 * * *$ & $-4.277^{* * *}$ \\
Observations & & & 323,261 & 323,261 \\
\hline Sensitivity & & & 0.577 & 0.478 \\
Specificity & & & 0.456 & 0.636 \\
C-stat (Area under ROC) & & & 0.520 & 0.595 \\
LR test M1 & & & $559 * * *$
\end{tabular}

*** $p<0.01, * * p<0.05, * p<0.1$ 
Table 40: PSI20

\begin{tabular}{lllll}
\hline Variables & Mean & Std. Dev. & M1 & M2 \\
\hline PSI20 & 0.004 & 0.064 & & \\
agecat1 (<30) & 0.385 & 0.487 & $-0.332^{* * *}$ & $-0.311^{* * *}$ \\
agecat3 (40-49) & 0.057 & 0.232 & -0.0711 & -0.0657 \\
Maori & 0.140 & 0.347 & & -0.188 \\
Pacific & 0.083 & 0.275 & & 0.0499 \\
Asian & 0.103 & 0.304 & & 0.0990 \\
Other & 0.119 & 0.324 & & $-0.260^{*}$ \\
NZDep (lower quintile) & 0.177 & 0.382 & & -0.0671 \\
NZDep (second quintile) & 0.180 & 0.385 & & -0.161 \\
NZDep (fourth quintile) & 0.217 & 0.412 & & -0.0915 \\
NZDep (upper quintile) & 0.228 & 0.419 & & -0.167 \\
Constant & & & $-5.383^{* * *}$ & $-5.257^{* * *}$ \\
Observations & & & 119,227 & 119,227 \\
\hline Sensitivity & & & 0.688 & 0.579 \\
Specificity & & & 0.386 & 0.510 \\
C-stat (Area under ROC) & & & 0.538 & 0.552 \\
LR test M1 & & & 7.54
\end{tabular}

*** $p<0.01, * * p<0.05, * p<0.1$ 


\section{Appendix E}

\begin{tabular}{ll}
\multicolumn{2}{l}{ Table 41: Ministry of Health Filters for NMDS } \\
\hline No. & Filter \\
\hline $\mathbf{1}$ & Non-treated patients \\
$\mathbf{2}$ & Error DRGs \\
$\mathbf{3}$ & Renal Dialysis \\
$\mathbf{4}$ & Same day chemotherapy and radiotherapy \\
$\mathbf{5}$ & Sleep apnoea \\
$\mathbf{6}$ & Lithotripsy \\
$\mathbf{7}$ & Colposcopies \\
$\mathbf{8}$ & Cytoscopies \\
$\mathbf{9}$ & ERCPs \\
10 & Colonoscopies \\
$\mathbf{1 1}$ & Gastroscopies \\
$\mathbf{1 2}$ & Bronchoscopies \\
$\mathbf{1 3}$ & Day case transfusions \\
$\mathbf{1 4}$ & Inconsistent stays \\
$\mathbf{1 5}$ & Well babies \\
16 & Mental health cases \\
$\mathbf{1 7}$ & DSS cases \\
$\mathbf{1 8}$ & Transfers \\
19 & A\&E day/short stay observations \\
$\mathbf{2 0}$ & Overseas patients \\
\hline
\end{tabular}

